Synthèses

\title{
Apprendre à innover dans un monde incertain
}

Concevoir les futurs de l'agriculture et de l'alimentation

E. Coudel, H. Devautour, C.T. Soulard, G. Faure, B. Hubert, coord.

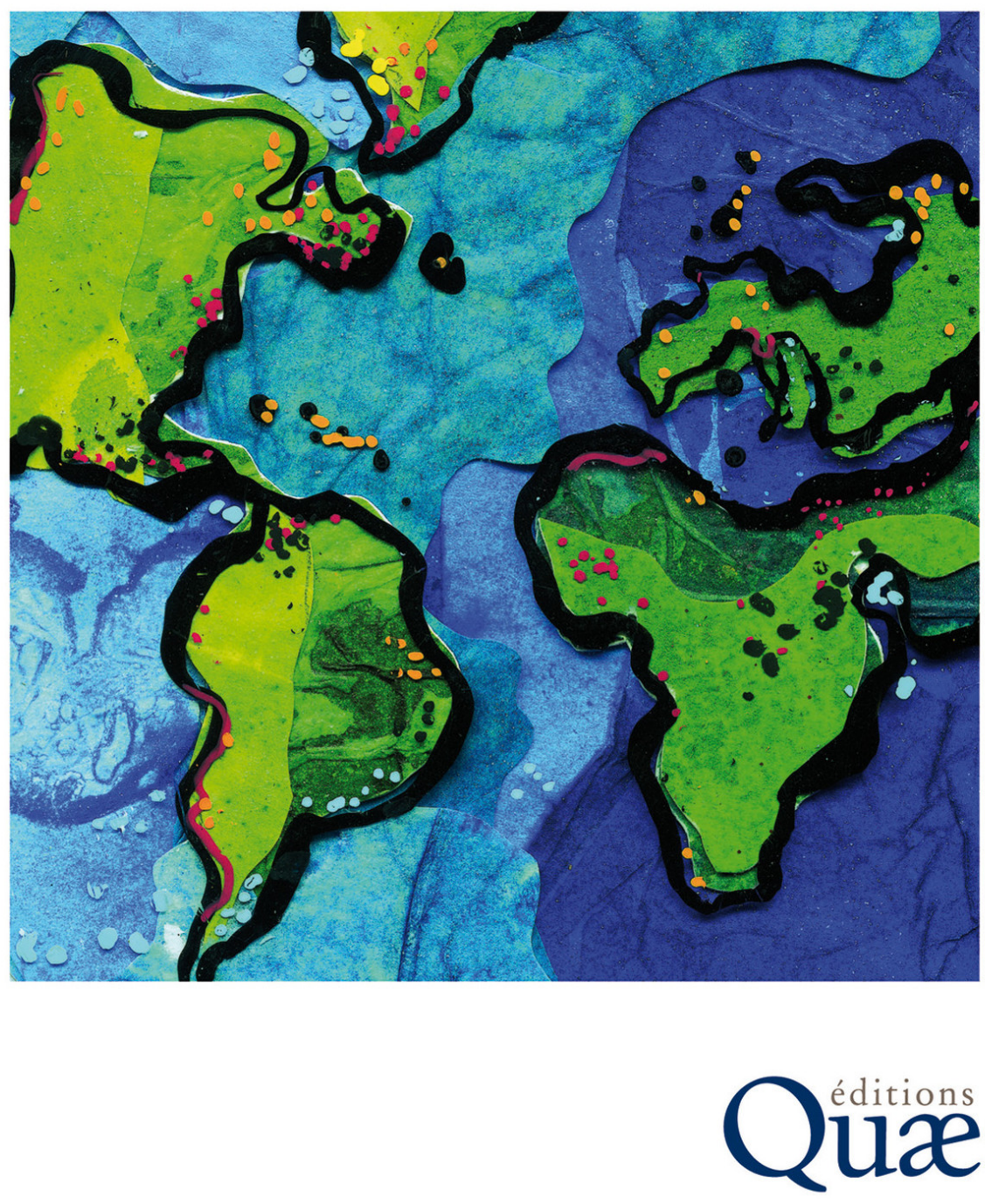




\section{Apprendre à innover dans un monde incertain}

Concevoir les futurs de l'agriculture et de l'alimentation

Émilie Coudel, Hubert Devautour, Christophe-Toussaint Soulard, Guy Faure et Bernard Hubert (dir.)

Éditeur : Éditions Quæ

Année d'édition : 2012

Date de mise en ligne : 30 janvier 2020

Collection : Synthèses

\section{Ooperatition ebooks}

http://books.openedition.org

Édition imprimée

Nombre de pages : 248

Référence électronique

COUDEL, Émilie (dir.) ; et al. Apprendre à innover dans un monde incertain : Concevoir les futurs de

l'agriculture et de l'alimentation. Nouvelle édition [en ligne]. Versailles : Éditions Quæ, 2012 (généré le 31 janvier 2020). Disponible sur Internet : <http://books.openedition.org/quae/21282>.

(c) Éditions Quæ, 2012

Creative Commons - Attribution - Pas d'Utilisation Commerciale - Pas de Modification 3.0 France - CC BY-NC-ND 3.0 FR 


\section{Apprendre à innover}

dans un monde incertain

Concevoir les futurs de l'agriculture et de l'alimentation 


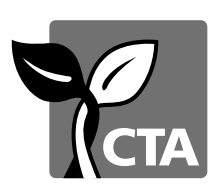

Le Centre technique de coopération agricole et rurale (CTA) est une institution internationale conjointe des États du Groupe ACP (Afrique, Caraïbes, Pacifique) et de I'Union européenne (UE). II intervient dans les pays ACP pour améliorer la sécurité alimentaire et nutritionnelle, accroître la prospérité dans les zones rurales et garantir une bonne gestion des ressources naturelles. II facilite l'accès à l'information et aux connaissances, favorise l'élaboration des politiques agricoles dans la concertation et renforce les capacités des institutions et communautés concernées.

Le CTA opère dans le cadre de l'Accord de Cotonou et est financé par I'UE.

\section{CTA}

Postbus 380

6700 AJ Wageningen

Pays-Bas

www.cta.int 


\section{Apprendre à innover dans un monde incertain Concevoir les futurs de l'agriculture et de l'alimentation}

E. Coudel, H. Devautour, C.T. Soulard,

G. Faure, B. Hubert, coordinateurs 


\section{Collection Synthèses}

Odorat et goût. De la neurobiologie des sens chimiques aux applications

Roland Salesse et Rémi Gervais, coordinateurs

2012, $550 \mathrm{p}$.

Comment I'herbe pousse. Développement végétatif, structures clonales et spatiales des graminées

Michel Lafarge, Jean-Louis Durand

2011, $184 \mathrm{p}$.

Grands paysages pédologiques de France

Marcel Jamagne

2011, 624 p.

Production durable de biomasse. La lignocellulose des poacées

Denis Pouzet

2011, 216 p.

La photosynthèse. Processus physiques, moléculaires et physiologiques

$2^{\mathrm{e}}$ édition

Jack Farineau, Jean-François Morot-Gaudry

2011, 412 p.

Les chapitres 1, 2, 3, 6, 8 et 9 ont été traduits en français par Anya Cockle.

Version anglaise :

Renewing innovation systems in agriculture and food:

how to go towards more sustainability?

(c) Wageningen Publishers, 2012

Version française :

(c) Éditions Quæ, CTA, 2012 ISBN (Quæ) : 978-2-7592-1858-5

ISBN (CTA) : 978-92-9081-505-1

ISSN : $1777-4624$

Le code de la propriété intellectuelle interdit la photocopie à usage collectif sans autorisation des ayants droit. Le non-respect de cette disposition met en danger l'édition, notamment scientifique, et est sanctionné pénalement. Toute reproduction, même partielle, du présent ouvrage est interdite sans autorisation du Centre français d'exploitation du droit de copie (CFC), 20 rue des Grands-Augustins, Paris 6 e. 


\section{Préface}

Aucun doute : la question de l'innovation, de ses processus et des effets qu'elle produit, est fondamentale, tant pour les institutions de recherche que pour les politiques et l'ensemble de la société.

En 1993, à l'initiative de la mission Économie du Cirad, trois institutions françaises de recherche, le Cirad, l'Inra et l'IRD ${ }^{1}$, organisaient à Montpellier un séminaire international sur le thème «Innovation et sociétés - Quelles agricultures ? Quelles innovations ? ». Les années 1990 étaient marquées par une mobilisation récente des sciences humaines et sociales au sein des institutions de recherche agronomique. La mission assignée à ces dernières était de mieux saisir et surmonter ce qui était perçu comme des résistances sociales et culturelles au changement, et de faciliter et accélérer ainsi la diffusion des résultats de recherche. Dans le même temps, les représentants de ces disciplines vivaient de manière inconfortable l'asymétrie de pensée et d'action entre sciences du vivant, dominantes, et sciences sociales, minoritaires. Ce séminaire était ainsi bienvenu pour faire un point sur les processus d'innovation se dissimulant derrière ces résistances et pour alimenter le dialogue entre disciplines.

Depuis lors, dans un contexte où les questions d'environnement et de paupérisation se font pressantes, l'actualité de la recherche agronomique internationale est marquée par la référence à une contribution au développement durable et aux objectifs du millénaire, même si leurs atteintes semblent hors de portée par rapport aux échéances qui avaient été fixées par la communauté internationale. Ce constat représente une motivation forte pour revisiter aujourd'hui les débats de l'époque. Comment mieux situer nos recherches et nos actions dans une perspective de changement et de rupture?

Quelles bonnes raisons avions-nous donc de relancer la réflexion 17 ans plus tard en organisant un symposium international ? L'initiative reposait sur quatre motivations. Il importait tout d'abord de mieux situer nos travaux dans une perspective de réflexion internationale et d'élargir la participation au-delà des seules institutions françaises. Dans un second temps, il y avait un véritable défi à dépasser le cadre conceptuel des sciences humaines et sociales, et à rechercher les fécondations vertueuses de l'interdisciplinarité. Ensuite, tout en reconnaissant l'irréductibilité du local, l'enjeu de regarder au-delà des dimensions locales, pour aborder la dialectique entre différents niveaux d'organisations fait écho à la dimension globale des questions du développement. Enfin, se confronter, au-delà de la recherche, aux acteurs

1. Office de la recherche scientifique et technique outre-mer (Orstom) à l'époque. 
du développement et de la formation devait permettre de mieux se positionner par rapport aux évolutions de la société.

Recherche et enseignement sont fortement ébranlés par les évolutions techniques, économiques et sociales et la montée des incertitudes soulignées quotidiennement par les médias. Les crises environnementales, économiques, financières, sociales, politiques sont devenues dans bien des cas le fil rouge guidant la réflexion et l'action. Elles soulignent l'ardent besoin de processus d'adaptation et de régulation fondés sur l'action collective, interrogeant le statut des connaissances et remettant en cause la toute puissance de l'expertise scientifique. L'innovation est toujours perçue comme un processus d'où naîtront les solutions pour demain. Mais la propension avérée de l'innovation à générer dans certains cas de l'exclusion ne relève pas seulement de la rhétorique et pose la question du lien entre innovation et équité, soulignée dans le rapport IAASTD ou le rapport de la Banque mondiale (2008).

De façon fondamentale, les évolutions actuelles interrogent les modèles de développement que nous véhiculons inconsciemment ou consciemment et notre foi inébranlable dans la portée positive de la différentiation et de l'innovation. $\mathrm{Ne}$ confondons-nous pas systématiquement l'innovation avec le développement ? Nos programmes ne sont-ils pas tous tournés vers le changement comme paradigme du progrès social ? N'est-ce pas le fondement même de l'action de la recherche finalisée que de s'accoler à l'innovation au travers du binôme invention-innovation ? En nous donnant comme intention de poser un regard sur l'innovation, nous ne pouvons ignorer ces questions.

Il nous a ainsi paru pertinent de revisiter nos modèles de développement au travers d'une réflexion sur le lien entre innovation et développement durable, et ce par l'analyse des effets des processus d'innovation en termes de «développement ». Le scientifique ne saurait se laisser piéger par une croyance irraisonnée dans les vertus de l'innovation. Déjà en 1993, Chauveau et Yung², en s'inspirant de Hirshman, avaient opposé deux visions de ces processus. D’une part, une rhétorique dite progressiste, ou encore scientiste, adossée à une conception diffusioniste de l'innovation, fait de cette dernière un facteur de progrès technique et social. D'autre part, une rhétorique dite réactionnaire, critique de l'innovation, dénonce la captation de ses bénéfices par les dominants ou encore la remise en cause des sociétés traditionnelles par les effets générés. Ce regard demeure extrêmement pertinent et utile.

Ce sont les objectifs et les défis du symposium ISDA - Innovation et développement durable, organisé par le Cirad, l'Inra et Montpellier SupAgro, que de développer des regards renouvelés et croisés sur l'innovation. L'ambition est de saisir comment s'opère le lien entre production de connaissance, apprentissage et innovation en faisant le lien avec cette fameuse finalité, le développement. Elle invite à aborder l'innovation elle-même comme objet de recherche et à faire le point sur les avancées scientifiques dans ce domaine. Elle permet de stimuler la réflexivité sur nos actions de recherche et de développement et de générer de nouvelles manières de

2. Chauveau, Yung, 1995, Innovation et Sociétés - V2 Les diversités de l'Innovation, actes du XIV e séminaire d'économie rurale, Inra-Cirad-Orstom, 13-16 septembre 1993, Montpellier, Editions CIRAD, $380 \mathrm{p}$. 
penser l'innovation. Et non des moindres, cette ambition favorise l'innovation au sein même du monde scientifique.

Ce symposium a effectivement fait émerger de nombreux débats entre communautés travaillant sur l'innovation. Réunissant plus de 500 acteurs de 65 pays, il constitue une nouvelle étape dans les réflexions sur l'innovation. Je suis convaincu que les fruits de ce symposium impacteront la programmation de nos institutions. Cet ouvrage témoigne de la richesse des débats et ouvre sur de nouveaux défis pour nos institutions de recherche et pour la conception de nouvelles politiques de développement.

Patrick Caron

Directeur général délégué à la recherche et à la stratégie du Cirad 


\section{Remerciements}

Ce livre est l'aboutissement d'un long processus d'articulation, de débats, de créativité. Merci à toutes les forces vives et innovantes qui se sont mobilisées pour faire vivre le symposium ISDA 2010 et pour capitaliser ensuite les nombreuses discussions qui y ont eu lieu.

Merci à toute l'UMR Innovation pour avoir lancé l'idée d'une rencontre pour rassembler les communautés qui travaillent sur l'innovation en agriculture et agroalimentaire et d'avoir porté ce projet jusqu'à son aboutissement.

Merci aux membres du comité scientifique pour avoir apporté leur vision, leurs conseils et leur esprit de synthèse : B. Hubert (Agropolis International, France), F. Aggeri (Ensmp, France), C. Almekinders (WUR, Pays-Bas), J. Berdegue (Rimisp, Chili), R. Buruchara (Ciat, Colombie), P. Caron (Cirad, France), B. Chevassus-au-Louis (ministère de l'Agriculture, France), O. Coomes (University of McGill, Canada), S. Dubuisson Quellier (Cnrs, France), M. Fonte (Université de Naples, Italie), A. Hall (LINK Ltd. \& CRT-RIU, UK), S. Hisano (University of Kyoto, Japon), K. Hussein (OCDE), P.-B. Joly (Inra, France), J. Kirsten (University of Pretoria, Afrique du Sud), J.-M. Meynard (Inra, France), D. Requier Desjardins (IEP Toulouse, France), P.A. Seck (Africa Rice, Bénin), R. Teulier (Cnrs, France).

Merci aux membres du comité scientifique local pour avoir contribué avec leur entrain tout au long du processus et pour avoir agréger leurs réseaux pour faire de cet événement une réussite : H. Devautour, E. Coudel, M. Antona, J.-M. Barbier, D. Bazile, E. Biénabe, E. Chia, D. Desclaux, G. Faure, P. Gasselin, P. Maizi, M. Piraux, C. Poncet, B. Prevost, S. Ridaura, C. Soulard, L. Temple, L. Temri, J.-M. Touzard, B. Triomphe, E. Valette.

Merci à tous ceux (surtout celles !) qui dans l'ombre, mais toujours efficaces, ont coordonné toute la logistique, la communication, les voyages : M.-F. Chazalette, B. Gillet, N. Kelemen, E. Grégoire, A. Rossard, C. Rollin, D. Delebecque, T. Erwin, P. Radigon, C. Rawski, P. LajousCausse. Un merci spécial à K. Agrawal et A. Cockle pour la traduction des chapitres de ce livre.

Merci à ceux qui ont accepté d'apporter leur énergie pour l'animation des sessions : C. Albaladejo, M. Barbier, P. Bonnal, M. Bonin, F. Bousquet, C. Bryant, E. Cheyns, N. Cialdella, Y. Chiffoleau, E. Doidy, M. Dosso, S. Dury, C. Ferraton, S. Fournier, C. Gary, F. Goulet, C. Harris, H. Hocdé, H. Ilbert, F. Jarrige, G. Kamau, P.-Y. Le Gal, V. Mathieu, A. Quinlan, R. Rajalahti, H. Rey-Valette, S. Ridaura, G. Ruivenkamp, D. Sautier, P. Tittonell, E. Torquebiau, A. Torre, S. de Tourdonnet, J. van den Berg, F. Wallet, A. Waters-Bayer.

Merci aux différents bailleurs pour leur soutien financier qui a notamment permis de faire venir autant de participants du Sud : le Centre technique agricole (CTA), le Fonds international de développement agricole (FIDA), Agropolis Fondation, le ministère de la culture et de la communication (Délégation générale à la langue française et aux langues de France), la Région Languedoc-Roussillon, l'agglomération de Montpellier. Merci au parrainage du ministère de l'Agriculture, de l'Agroalimentaire et de la Forêt, du ministère des Affaires étrangères et européennes, et du ministère de l'Écologie, du Développement durable et de l'Énergie. Merci à la Banque mondiale et à l'Organisation de coopération et de développement économique (OCDE) pour leur participation.

Merci finalement aux plus de 500 participants d'ISDA 2010 pour leur enthousiasme, leurs idées, leur volonté d'échanger. 


\section{Table des matières}

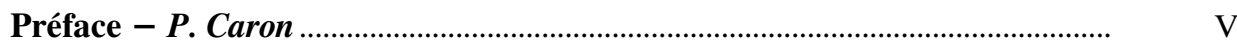

Remerciements ................................................................................................... VIII

Introduction. Repenser l'innovation pour le développement durable G. Faure, E. Coudel, C.T. Soulard, H. Devautour ...................................................

L'innovation pour quel développement ?........................................................ 3

La recherche sur l'innovation évolue pour prendre en compte la complexité ......................................................................................................

Nouvelles questions dans un monde incertain ................................................. 11

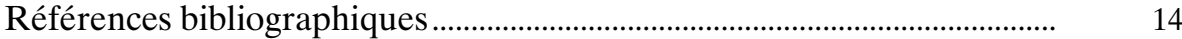

\section{PARTIE 1. REPENSER L'INNOVATION DANS UN MONDE INCERTAIN}

\section{Chapitre 1. Normes gouvernant l'innovation agricole}

L. Busch

Les normes : construire des réalités

Avant la recherche agricole : l'exploitant expérimentateur.......................... 21

La recherche agronomique en tant que bien public, 1600-1980 ................. 22

La recherche agronomique en tant que bien privé, de 1980 environ à nos jours.

Encadré 1.1. Semences : du bien marchand au bien commun

$P$. Lemmens et G. Ruivenkamp.

Les supermarchés, les transformateurs et la révolution des normes......... $\quad 30$

La tempête qui se profile..................................................................................... 32

Encadré 1.2. Nouvelles normes d'évaluation de la recherche :

le cas du système de riziculture intensive - S.P. Chebrolu .

Élargir le champ d'investigation de la recherche agronomique

Encadré 1.3. Exemple de réorientation de la recherche agronomique au bénéfice de petits producteurs au Brésil - E. de Carvalho Noya,

B. Roux, G. Majella Bezerra Lopes

Références bibliographiques 
Chapitre 2. Systèmes d'innovation : du concept à la pratique émergente B. Triomphe et R. Rajalahti.

Le concept de système d'innovation et son origine.

Faire passer l'approche des systèmes d'innovation au stade opérationnel.

Encadré 2.1. Stimuler l'innovation locale et la recherche conjointe orientée par les agriculteurs

Encadré 2.2. Papa Andina et l'approche participative des chaînes de marchés.

Encadré 2.3. Le projet national indien d'innovation agricole (NAIP)

Défis rencontrés dans la mise en œuvre des systèmes d'innovation agricole

Conclusions et perspectives

Références bibliographiques

Chapitre 3. De quels types d'entrepreneurs innovants avons-nous besoin ? A. Hall et K. Dorai.

Encadré 3.1. Quelques exemples d'esprit d'entreprise

Les systèmes d'innovation : au-delà des concepts ?

Les sources alternatives de perturbation : l'esprit d'entreprise

et le développement.

Que trouve-t-on dans les zones d'ombre du radar?.

Conclusions et conséquences en matière de politiques

Conséquences pratiques

Références bibliographiques

\section{PARTIE 2. Apprendre À INNOVER AUJOURD'HuI}

Chapitre 4. Innover dans les systèmes de culture et de production J.M. Meynard .

Logiques agronomique, économique et sociale des systèmes agricoles intensifs en intrants : un exemple en France.

Quelles marges de manœuvre pour faire évoluer les systèmes intensifs en intrants?.

Outils et démarches pour la reconception des systèmes agricoles :

quelques pistes de travail.

Encadré 4.1. Conception «pas à pas » d'un système de production agroécologique en Picardie (Mischler et al., 2009)...

Conclusion

Références bibliographiques 
Chapitre 5. Innovation et insertion sociale, réduire la vulnérabilité des populations rurales - D. Requier-Desjardins

Retours sur quelques concepts

Encadré 5.1. Horticulture et approche par les capacités au Cameroun -

L. Parrot, P. Pédelahore, H. De Bon, R. Kahane..

L'innovation agricole est-elle un facteur de réduction de la pauvreté et de la vulnérabilité, et de durabilité des trajectoires de développement?

Des innovations pour réduire la pauvreté rurale?

Encadré 5.2. Syal et développement rural : l'Alliance des agro-industries rurales de la Selva Lacandona, Chiapas $-F$. Boucher, D. Requier-

Desjardins, V. Brun

Encadré 5.3. Enseignements tirés du « Panorama Andino » sur l'innovation rurale dans les Andes - E. Fernandez-Baca, M. Paz Montoya,

N. Yañez.....

Conclusion.

Références bibliographiques.

\section{Chapitre 6. Innovations de marché axées sur la qualité : dimensions} sociales et d'équité - E. Biénabe, C. Bramley, J. Kirsten.

Innovations axées sur la qualité et implications pour les petits agriculteurs

Les effets des démarches de qualité pour les petits agriculteurs :

des observations peu concluantes

Encadré 6.1. Apprendre à exporter : partenariats agriculteurs-exportateurs au Kenya - M. O. Bolo

Encadré 6.2. Les normes du commerce équitable comme moyens

d'innover ? - A. Loconto et E. Simbua

Encadré 6.3. Construction sociale des normes dans l'agriculture biologique et inclusion des petits agriculteurs en Argentine - M. Mateos et G. Ghezán.

Conclusion.

Références bibliographiques.

\section{Chapitre 7. Innovations et gouvernance des territoires ruraux}

Les modèles de développement régional et territorial.

Les politiques de développement par l'innovation

Encadré 7.1. Les systèmes agroalimentaires localisés (Syal).

Encadré 7.2. Un exemple d'innovation socio-territoriale : la transition agroécologique au Brésil - M. Piraux et P. Bonnal.

Quelle gouvernance pour faire émerger l'innovation dans les espaces ruraux et périurbains?

Encadré 7.3. Co-construction d'un guide de la gouvernance territoriale -

H. Rey-Valette et E. Chia 
Conclusion

Références bibliographiques

\author{
Partie 3. ConceVoir des futurs \\ POUR L'AGRICULTURE ET L'AGROALIMENTAIRE
}

\title{
Chapitre 8. L'agrobiodiversité, vers des instruments de protection
} innovants $-J$. Santilli

L'agrobiodiversité : un concept en construction

Les diverses contributions de l'agrobiodiversité

L'agrobiodiversité et les instruments juridiques ....

Encadré 8.1. Quand des paysans de communautés pauvres combinent sélection variétale participative et gestion in-situ et ex-situ (Honduras) H. Hocdé, J.C. Rosas, R. Araya.

Encadré 8.2. Le rôle des curadoras mapuches dans la conservation des variétés de quinoa $-M$. Thomet et $D$. Bazile

Encadré 8.3. De la ressource phytogénétique au patrimoine culturel : les bases sociales de la gestion de l'agrobiodiversité en Amazonie brésilienne - L. Eloy et L. Emperaire.

Conclusion

Références bibliographiques

Chapitre 9. Politiques pour promouvoir l'innovation, le cas

de la Méditerranée - K. Hussein et K. El Harizi.

Les défis globaux et régionaux en matière d'agriculture et d'alimentation : l'impératif de l'innovation

Les concepts clés de l'innovation et leurs implications en matière de politiques, de recherche et d'action.

Les cadres et stratégies internationaux pour favoriser l'innovation dans les sciences et les technologies

Encadré 9.1. Un exemple de réseau interrégional pour stimuler

l'innovation agricole

Le Plan Vert Maroc, exemple d'une stratégie nationale d'innovation .....

Programmes pour stimuler l'innovation agricole à l'échelon local.

Implications pour la promotion de l'innovation en Méditerranée

Encadré 9.2. Apprentissages pour la gestion participative de l'irrigation au Maroc - Z. Kadiri, M. Kuper, M. Errahj.

Encadré 9.3. Innovations institutionnelles de la nouvelle stratégie céréalière en Tunisie $-R$. Khaldi

Conclusion

Références bibliographiques 
Chapitre 10. Rôle d'une gouvernance redynamisée de l'agriculture

africaine - P.A. Seck, A. Diagne, I. Bamba.

Étiologie de la mauvaise prise en charge des questions agricoles.

Formation des acteurs et faible niveau d'organisation.

Transformation qualitative de l'agriculture africaine

Organisations professionnelles bien formées et informées

Mise en place d'observatoires des filières agricoles

Gouvernance de la recherche agricole

Encadré 10.1. Plates-formes pour l'innovation dans le secteur de l'élevage en Éthiopie - K. Ergano.

Les systèmes nationaux de recherche agronomique

Les dispositifs régionaux de coordination des recherches

Pour une programmation concertée des priorités de recherche

Encadré 10.2. Recherche-action-en-partenariat, une démarche de réconciliation entre recherche et société - M. Blanchard, E. Chia, M. Koutou, E. Vall

Repenser le rôle de l'État dans le secteur agricole.

Conclusion

Références bibliographiques.

Conclusion. En route, mais laquelle?

B. Hubert, E. Coudel, O. Coomes, C.T. Soulard, G. Faure, H. Devautour.

Revisiter les chemins habituels de l'innovation.

Confronter nos visions de l'agriculture pour en explorer de nouvelles....

Engager les transitions pour générer le changement.

Innover, c'est d'abord interagir pour apprendre.

Le chercheur, acteur du changement ?

Références bibliographiques

Sigles 



\title{
Introduction
}

\section{Repenser l'innovation pour le développement durable}

\author{
Guy Faure, Emilie Coudel, \\ Christophe T. Soulard, Hubert Devautour
}

Le monde est secoué par une crise systémique aux multiples facettes. À des changements structurels et continus comme le changement climatique, la pression accrue sur les ressources renouvelables et la croissance démographique (encore forte en Afrique), s'est combinée une crise économique violente aux conséquences imprévisibles avec une aggravation de la pauvreté, contraction des marchés d'export, resserrement des fonds de crédit et de développement. Dans ce contexte, l'agriculture fait face à un futur incertain, particulièrement dans certaines régions du globe, avec l'émergence de modèles de développement différenciés se traduisant par une agriculture familiale de plus en plus fragilisée et l'essor d'une agriculture capitalistique. Toutefois, à long terme, cette crise systémique peut aussi offrir de nouvelles opportunités. Elle conduit à une situation de rupture, remettant en cause des paradigmes considérés jusqu'alors comme allant de soi. Elle a révélé la vulnérabilité des systèmes agricoles et agro-alimentaires et a mis en évidence un besoin d'innovation pour renouveler les modèles de développement. À une époque de grande incertitude, alors que les valeurs et les normes changent, nos sociétés doivent se montrer créatives et réinventer les modes de production, de transformation et de distribution des produits agricoles dans une vision à long terme qui prenne en compte les territoires et les sociétés qui y vivent, mettant au centre des préoccupations le concept de durabilité.

Plusieurs systèmes agricoles et agroalimentaires ont déjà prouvé leur capacité à promouvoir un développement durable en se fondant sur des principes de production agro-écologiques (de Schutter, 2011) ou en favorisant des systèmes alimentaires locaux (Muchnik et al., 2010). Ces systèmes innovants sont encore peu répandus et ils évoluent en concurrence ou en complémentarité avec des systèmes productivistes dominants, dont certains revendiquent également la prise en compte d'une durabilité environnementale. Ces systèmes agricoles innovants prennent des formes variables selon qu'ils émergent dans des pays où l'agriculture est fortement consommatrice de capitaux, d'intrants chimiques et d'énergie fossile, ou des pays où l'agriculture a peu accès à ces ressources, se traduisant par une productivité du travail faible. 
S'il est nécessaire de rendre compte, d'échanger et de capitaliser ces expériences innovantes, il est encore plus urgent de faire émerger un nouveau paradigme pour concevoir autrement les effets de développement de l'agriculture. Le renouvellement des systèmes agricoles et agroalimentaires ne signifie pas seulement définir de nouvelles solutions techniques et/ou organisationnelles. Il interroge le statut des connaissances nécessaires à un développement durable et remet en cause le monopole de la connaissance scientifique par rapport aux autres formes de connaissance. Il oblige à transformer les processus d'innovation en créant de nouvelles articulations entre recherche, acteurs économiques, acteurs de la société civile et décideurs politiques. Désormais, la recherche agricole doit repositionner sa contribution à l'innovation, sachant que l'agriculture n'est plus interrogée pour sa seule fonction de production, mais aussi dans ses interactions avec l'environnement et la société dans son ensemble. Les questions sont aujourd'hui « agriculture et santé », « agriculture et environnement », « agriculture et énergie », " agriculture et activités rurales »... Pour comprendre ces changements et ce qu'ils impliquent pour la recherche, une réflexion collective doit s'engager.

Différents ouvrages récents proposent de nouvelles directions pour encourager l'innovation. Ainsi, les ouvrages Innovation Africa (Sanginga et al., 2009) ou Farmer first revisited (Scoones et al., 2009) insistent sur la nécessité de promouvoir des collaborations entre les paysans, la recherche, les services de conseil et le secteur privé pour créer des connaissances utiles pour l'action et améliorer les technologies adaptées aux conditions des paysans. Le rôle du marché comme élément pouvant tirer les innovations est affirmé de plus en plus nettement. L'accent est mis sur le renforcement des capacités des acteurs et donc sur la formation et le conseil, le renforcement du capital social et donc des réseaux, la création de nouveaux arrangements institutionnels incluant la promotion de plateformes multiacteurs, et l'élaboration de politiques publiques adaptées. L'ouvrage La rechercheaction en partenariat de Faure et al. (2010) met l'accent sur de nouvelles manières de mener des recherches en donnant une place plus importante aux interactions entre acteurs.

Les débats scientifiques sont vifs et portent sur la place que peut occuper chaque acteur dans le processus d'innovation ou sur l'identification et la caractérisation des facteurs qui déterminent les innovations, et donc avec des positions contrastées sur le type d'innovations nécessaires pour permettre un développement durable. C'est pourquoi nous avons souhaité initier une confrontation et une mise en commun de ces réflexions lors du symposium international, Innovation et développement durable dans l'agriculture et l'agroalimentaire (ISDA), qui s'est tenu en juin 2010, à Montpellier (France) rassemblant des chercheurs de disciplines variées, des acteurs du développement et des décideurs politiques, des pays du Nord et du Sud. Cet ouvrage restitue ces réflexions, qui analysent des expériences visant à promouvoir l'innovation, tirent des leçons des succès et des échecs de ces expériences et font émerger de nouvelles perspectives scientifiques et politiques pour des systèmes d'innovations capables de contribuer à un développement durable.

Les chapitres de cet ouvrage sont l'expression de positions différentes face aux processus d'innovation, des réflexions d'analystes fin connaisseurs des dynamiques d'innovation, d'institutionnels impliqués dans l'accompagnement de l'innovation, de 
chercheurs ayant analysé et participé à des innovations sur le terrain. Les auteurs se sont appuyés sur les nombreuses présentations et discussions menées au cours du symposium ISDA, dont certains cas illustreront leur propos au fil du texte ${ }^{1}$.

\section{W'innovation pour quel développement ?}

\section{Le développement sans cesse interrogé}

Travailler sur l'innovation et ses effets de développement amène à s'interroger sur le sens que les acteurs donnent à la notion de développement. Or la définition du développement ne va pas de soi. Une première définition, largement contestée mais toujours bien présente dans les débats actuels, affirme que « le développement, c'est la croissance économique » qui se mesure principalement par le produit intérieur brut (PIB) des nations. Des déclinaisons sont possibles en prenant en compte d'autres critères comme la justice sociale : « le développement, c'est alors une croissance économique équitablement répartie ». La pensée économique sur le développement a été marquée par la nécessaire évolution des sociétés grâce à la croissance. Par exemple, Rostow (1979) identifie 5 phases par lesquelles les sociétés doivent passer pour s'approcher des sociétés occidentales, dites " développées » : (1) la société traditionnelle agricole, (2) l'extension des échanges avec les premiers changements des techniques et des mentalités, (3) le décollage tiré par une croissance cumulative, (4) la « marche vers la maturité » où le progrès s'étend à toutes les activités, (5) l'avènement de la société de consommation de masse. Une telle définition du développement est marquée par la notion de progrès qui intègre l'idée d'une visée clairement définie, donc partagée par une large partie de la société, et vers laquelle il s'agit de cheminer pas à pas. Son usage caractérise une période de grandes planifications des interventions techniques et de croyance dans l'effet globalement positif de ces techniques.

Dès les années 1970, «Le Club de Rome » dénonce les dangers d'une croissance économique et démographique débouchant sur l'épuisement des ressources naturelles. Le modèle de développement basé sur l'accumulation des richesses est alors contesté. François Perroux (1963) propose une définition plus sociale : « le développement est la combinaison des changements mentaux et sociaux d'une population qui la rendent apte à faire croître, cumulativement et durablement, son produit réel global ». Une des alternatives proposées au développement économique est celle de Maurice Strong, secrétaire général de la conférence de Stockolm, en 1972, qui parla d' "éco-développement », dans le sens d'une utilisation prudente des ressources et d'une valorisation des savoirs issus du tiers-monde. Cette proposition, qui a été reprise par Ignacy Sachs (1980), est devenue centrale dans les politiques du programme des Nations unies des années 1980. À cette même époque, le futur prix Nobel d'économie, Amartya Sen (1989, 1999), propose une relecture de la pauvreté au jour des besoins fondamentaux, des capacités et des libertés dont jouissent les

1. Les actes du symposium sont accessibles à l'adresse : http://hal.archives-ouvertes.fr/ISDA2010. 
individus, introduisant la notion de développement humain (plus tard formalisé par l'IDH, indicateur de développement humain). Lors de la conférence de Rio en 1992, ces idées convergent au sein du concept de développement durable en s'appuyant sur la célèbre définition de Brundtland : «le développement durable est un développement qui répond aux besoins du présent sans compromettre la capacité des générations futures à répondre aux leurs » (rapport Notre avenir à tous de la Commission mondiale sur l'environnement et le développement, 1987). Ce nouveau paradigme met en évidence la multiplicité des dimensions du développement. Les dimensions les plus évoquées sont celles de l'économique, du social et de l'environnemental. Mais il est possible de prendre en compte d'autres dimensions, également largement citées, comme celle du culturel ou de la gouvernance.

Ainsi, le développement devient la mise en œuvre d'un projet de société. La nature de ce projet n'est pas définie a priori en référence à un ou des modèles extérieurs. Les objectifs peuvent être différents d'une société à une autre, les chemins pour parvenir à atteindre les objectifs ne sont pas uniques mais divers. Ce qui importe, c'est la flexibilité, la résilience ou la réversibilité des systèmes économiques et sociaux en place afin de ne pas compromettre les capacités des sociétés à assurer leur futur.

\section{Le développement en agriculture}

Le secteur agricole est bien sûr marqué par cette pensée sur le développement. Pendant des décennies l'agriculture a été pilotée par l'objectif d'augmenter la production en favorisant d'une part un accroissement des surfaces cultivées par unité de travail ou de capital et d'autre part un accroissement des rendements des cultures et des animaux d'élevage. Ce modèle a été nommé la « révolution verte » dans les pays des Suds ou l'agriculture intensive dans les pays des Nords. La prise en compte d'autres dimensions liées aux territoires, aux filières ou chaînes de valeurs, ou aux systèmes alimentaires, n'a émergé que progressivement, en même temps que se révélaient les limites d'un modèle positiviste et productiviste. Le concept de multifonctionnalité de l'agriculture apparu dans les années 1990 en est l'illustration. Il reconnait que l'agriculture, au-delà de sa fonction de production, joue un rôle important dans la construction d'un territoire, participe à la gestion des ressources renouvelables, génère des emplois et contribue à forger une culture locale. Ainsi progressivement, d'autres modèles agricoles émergent, fondés sur des principes novateurs comme l'agroécologie (Altieri, 2002) ou cherchant à promouvoir de nouveaux circuits courts de commercialisation ou de nouvelles formes d'équité dans les marchés (Colonna et al., 2011).

Ce qui frappe l'observateur, c'est que ces nouveaux modèles agricoles, même s'ils progressent dans les esprits et plus timidement sur le terrain, ne semblent pas remplacer l'ancien modèle. Au contraire, différents modèles peuvent coexister sur un même territoire. Au Brésil par exemple, cohabitent depuis des décennies une agriculture familiale soutenue par l'État et une agriculture capitaliste où la finance prend un poids de plus en plus important. En Europe, le modèle fondé sur l'exploitation familiale devient insuffisant pour concevoir une politique agricole sans tenir compte de l'apparition d'investissements privés dans le secteur de la production comme de l'importance prise par la pluriactivité des ménages agricoles et ruraux. En Afrique, le land-grabing (l'accaparement des terres) est la matérialisation brutale 
de ces évolutions rapides, où de grandes entreprises privées ont la priorité sur le foncier face aux communautés traditionnelles. Si la cohabitation de ces modèles sur un territoire est un fait observable, il n'est par contre pas facile d'en évaluer la dynamique et les effets. Observe-t-on des conflits entre modèles, notamment pour l'accès aux ressources ? Quelles complémentarités, notamment sur des marchés différenciés ? Quelles synergies, par exemple dans la construction de nouveaux marchés ? Cette cohabitation peut aussi s'exprimer au sein même des organisations, avec par exemple une organisation de producteurs vendant une partie de ses produits sur un marché de niche et une autre partie dans le circuit de la grande distribution. Elle se manifeste aussi au niveau des consommateurs qui peuvent acheter le même jour un produit labélisé « commerce équitable » dans une boutique de producteurs et un autre produit bon marché dans un commerce « hard-discount ».

La diversité des modèles interroge la place des agriculteurs dans le développement agricole. Dans un sens proche de ce qu'est la «capabilité » pour Sen (1999), le développement peut correspondre à une augmentation de la capacité des agriculteurs, d'une part à définir une visée qui leur soit propre, d'autre part à obtenir et à mettre en œuvre les moyens d'atteindre cette visée. Il contribue à améliorer leur autonomie. Toutefois, ainsi énoncée, cette notion n'est pas exempte d'ambiguïté. En effet, si des collectifs d'agriculteurs peuvent se constituer et s'inscrire dans un tel processus, rien ne garantit qu'ils contribuent à la construction d'un bien considéré comme commun par le reste de la société. On trouve ici une conception «négociée » du développement où la définition de ce que doit être l'agriculture engage des acteurs divers, aux exigences variées, qu'ils soient agriculteurs ou non (Compagnone et al., 2009).

\section{La place de l'innovation dans le développement}

L'innovation est au cour des réflexions sur le développement quand elle n'est pas tout simplement perçue comme le moteur du développement. Schumpeter (1934) est le premier qui a théorisé cette innovation permettant à l'entrepreneur de construire un avantage comparatif par rapport à la concurrence et donc de générer des profits. Selon lui, l'innovation est la combinaison nouvelle de facteurs de production qui peut s'exprimer par la confection d'un nouveau produit, une nouvelle manière de produire, la construction de nouveaux débouchés, ou l'accès à de nouvelles ressources. L'innovation est une invention qui a trouvé son marché et donc qui s'inscrit dans un système de production. Depuis, de nombreux travaux visent à caractériser l'innovation qui, par exemple, peut-être incrémentale ou radicale, technique ou de processus, tirée par le marché (innovation pull) ou par l'offre technologique (innovation push). Il est classique de dire que l'innovation peut être de nature variée : technique, économique, organisationnelle, sociale, etc. De manière plus précise, elle est généralement composite : toute innovation technique va le plus souvent de pair avec une innovation organisationnelle dans laquelle on pourrait dire qu'elle est enchâssée. Ce constat amène à prendre en compte le concept d'innovation socio-technique ${ }^{2}$, l'objet technique étant appréhendé à travers les usages qui en sont faits et les liens sociaux que ses usages génèrent, modifient, ou détruisent

2. L'analyse socio-technique analyse l'objet dans son milieu social et se place à l'endroit précis où l'innovation se situe (Akrich et al., 1988). 
(Flichy, 1995). L'innovation peut se greffer sur des systèmes anciens ou constituer des ruptures, être d'origine exogène et portée par le monde des techniciens, ou endogène et portée par le monde des paysans. Elle peut émerger dans des contextes très divers et elle ne se comprend qu'en analysant la société globale et le contexte dans lequel elle se développe. Les innovations ont une histoire : elles naissent, se développent et meurent. Ce sont donc des instruments de stratégies pour atteindre des objectifs ou conforter les positions de certains acteurs, et des enjeux de pouvoir et de négociation au sein de la société, en rapport avec les effets de développement visés par les différents secteurs de la société.

À une autre échelle et dans les temps actuels, l'Europe affirme voir son développement dans une société de la connaissance et de l'innovation, seule à même de maintenir la compétitivité des pays et donc la prospérité des sociétés. L'innovation est mise à l'agenda de nombreux acteurs (décideurs politiques, chefs d'entreprise, institution de recherche, etc.) car elle s'inscrit bien dans un contexte de production plus complexe et incertain, où c'est la combinaison des dimensions du changement - matérielles, sociales, intellectuelles - qui doit permettre une adaptation permanente à un contexte instable qu'elles contribuent en même temps à modifier. Mais si l'innovation n'est pas simplement technologique et se réfère généralement à des processus complexes, tout changement au sein d'une organisation, voire d'une société, n'est pas une innovation. L'innovation est un processus visant intentionnellement l'émergence de la nouveauté et son appropriation par des acteurs de la société. L'innovation nécessite un projet de changement et une interaction entre des acteurs promouvant ce changement et des acteurs s'appropriant ce changement. Olivier de Sardan (1993) illustre bien la complexité du phénomène par la définition suivante de l'innovation « une greffe inédite, entre deux ensembles flous, dans une arène, via des passeurs ».

L'innovation interroge ainsi directement les modèles de développement que nous véhiculons, inconsciemment ou consciemment. Yung et Chauveau (1993) distinguent une rhétorique progressiste et scientiste de l'innovation comme source de progrès technique et social dans une vision diffusionniste, d'une rhétorique réactionnaire et critique de l'innovation qui dénonce la captation des bénéfices par les acteurs dominants au détriment des structures des sociétés traditionnelles. Au-delà des améliorations des performances des acteurs concernés par l'innovation, la question des conséquences de l'innovation sur les autres acteurs, en termes d'inclusion ou d'exclusion est donc centrale. Le progrès technique promu dans le secteur agricole, visant à améliorer la productivité de la terre et du travail, a mis à mal le devenir des petits agriculteurs dans tous les pays du monde (Röling, 2009).

\section{" La recherche sur l'innovation évolue pour prendre en compte la complexité}

La recherche a toujours joué un rôle important pour comprendre et promouvoir l'innovation. N'est-ce pas le fondement même de son action ? Toutefois, les pratiques de recherche ont évolué pour mieux prendre en compte la complexité croissante des problèmes auxquels sont confrontées les sociétés. Cette évolution repose 
sur une remise en cause progressive (et partielle) de son mode de production des connaissances et de sa façon de prendre en compte la demande dite "sociale » (quels acteurs, avec quels objectifs, quelles conséquences ?), menant à une manière différente d'envisager l'innovation. L'innovation a ainsi été successivement caractérisée dans les travaux des chercheurs en mettant l'accent sur le comportement des individus, les interactions entre individus (réseaux), puis les interactions entre les organisations. Nous retraçons cette évolution notamment dans le domaine agricole et agroalimentaire.

\section{Le rôle de l'agriculteur dans le processus d'innovation}

Jusqu'aux années 1980, les travaux de recherche mettent l'accent sur le producteur et son environnement pour analyser l'innovation. Rogers (1983) montre l'attitude différente des producteurs face au changement et élabore une typologie des innovateurs (les novateurs non intégrés, les dynamiques et leaders d'opinion, les attentistes, les marginalisés). Il montre que la diffusion de l'innovation est non linéaire et considère qu'elle correspond à une courbe en $\mathrm{S}$ avec en abscisse le temps et en ordonnée le nombre d'adoptants. Mais ce modèle diffusionniste ne prend pas en compte le fait que les conditions, liées à l'exploitation ou à l'environnement de l'exploitation, favorisant ou défavorisant l'innovation considérée, ne sont pas les mêmes pour tous. Comme le souligne Olivier de Sardan (1993), la diffusion de l'innovation, notamment dans les premières phases, dépend aussi des porteurs qui peuvent la véhiculer en fonction de leur position au sein de la société et des intérêts qu'ils perçoivent. Mendras et Forsé (1983), quant à eux, proposent cinq facteurs permettant d'évaluer l'«adoptabilité » des innovations, à savoir : l'avantage relatif apporté par l'innovation par rapport à la situation initiale, sa compatibilité par rapport au système en place, sa plus ou moins grande complexité, son « essayabilité » dans le contexte de l'acteur concerné, son observabilité chez autrui. Ces facteurs traduisent une prise en compte du degré de complexité et du niveau de risque par les producteurs. Mais la question n'est pas tant de savoir si l'innovation est compatible avec le système de production en place ou si le producteur peut s'adapter à l'innovation proposée. L'enjeu est de saisir la double relation qui se met en place : comment l'innovation modifie l'exploitation et comment le producteur incorpore en l'adaptant l'innovation?

Chauveau et al. (1999) relativisent cette façon de penser l'innovation qui met l'accent sur l'individu en insistant sur les interactions entre l'individu et son environnement. Ainsi, ils précisent que (i) l'offre et la demande d'innovation sont construites sur des interactions entre les acteurs autour d'enjeux techniques ; (ii) les innovations cheminent à travers des réseaux composites tenant compte de l'hétérogénéité des unités socio-économiques ; (iii) les relations entre innovation et environnement économique, social et politique sont des processus qui ne sont pas linéaires.

\section{La remise en cause du modèle diffusionniste}

Sur la base de ces travaux, le modèle dominant linéaire de transfert de connaissances et de technologies, appelé modèle diffusionniste, a été questionné dans sa capacité à apporter des réponses à des problèmes complexes. Il reste cependant encore vivace : 
il n'est pas rare que des chercheurs des sciences de la nature demandent l'appui des sciences humaines et des services de conseil pour diffuser des techniques mises au point en laboratoire ou en station expérimentale. Il n'en reste pas moins que les débats à propos de la nécessaire évolution du positionnement de la recherche dans le processus d'innovation ont été vifs. Le cas de la recherche dans les pays du Sud est illustratif des évolutions observées. Dès les années 1980, les approches de recherche-développement qui visent à améliorer les résultats des exploitations en tenant compte des réalités rurales ont donné lieu à une abondante littérature.

Jouve et Mercoiret (1987) définissent la recherche-développement comme «l'expérimentation en vraie grandeur et en concertation étroite avec les agriculteurs des améliorations techniques, économiques et sociales de leurs systèmes de production et des modalités d'exploitation de leur milieu ». Elle cherche à créer une relation triangulaire réciproque entre la recherche, la vulgarisation et les agriculteurs à tous les stades du processus de transformation des conditions de production. Les approches francophones de la recherche-développement et celles anglo-saxonnes du Farming System Research se rejoignent sur les principes de base (Jouve, 1994) : volonté de prendre en compte les conditions réelles de la production, approche systémique de la complexité des réalités agraires, conceptualisation de l'exploitation comme un système, analyse des pratiques des agriculteurs, participation paysanne au processus d'amélioration des exploitations, nécessité d'un contexte politique et social favorable à l'innovation.

Des critiques fortes ont été faites à la recherche-développement portant sur le coût en temps et en argent des phases de diagnostic, sur la faiblesse des recommandations en termes d'utilité pour l'action, et sur un risque d'instrumentalisation des producteurs au cours du processus d'expérimentation. Ces critiques ont généré des évolutions visant à promouvoir un approfondissement des démarches de recherche-action visant un processus d'apprentissage individuel et collectif. En milieu anglophone les recherches ont également évolué. À la critique des projets Farming System Research des années 1970-1980 (Norman et Collinson, 1985) ont succédé les méthodes accélérées de recherches participatives (Chambers et al., 1989 ; Scoones et al., 1994) qui mettront progressivement l'accent sur le diagnostic rapide (Rapid Rural Appraisal), la participation (Participatory Rural Appraisal), puis plus récemment sur les processus d'apprentissage (Participatory Learning Action), comme le montrent Lavigne Delville et al. (2000).

\section{Les réseaux d'innovation}

Progressivement, la réflexion sur l'innovation s'est ainsi centrée sur l'analyse des interactions entre les individus, s'intégrant dans des réseaux ou agissant au sein d'organisations. Le concept de capital social caractérise les réseaux entre acteurs, et en particulier la forme, la densité, et l'intensité des relations. Cette approche considère que le potentiel de développement d'un individu ou d'un groupe, et donc la capacité à innover, est lié à son capital social (Coleman, 1988). Dans les recherches sur l'innovation, l'accent est de plus en plus mis sur les processus d'apprentissage au niveau individuel, mais surtout au niveau des groupes et des organisations afin de produire des connaissances utiles pour provoquer un changement désiré. Dans le 
cadre des organisations, Argyris (1995) élabore ainsi les théories de l'apprentissage en simple boucle (changement de pratiques) ou en double boucle (changement de valeurs). Les notions de communauté de pratiques (Wenger, 1998) permettent de comprendre comment des individus, amenés à échanger fréquemment sur une question donnée, apprennent dans leur activité professionnelle et génèrent de nouveaux savoirs validés par cette communauté.

Dans le monde agricole, Darré (1996) montre que des réseaux de dialogue et de travail d'agriculteurs sont à la source de l'élaboration des savoirs. Ces réseaux sont constitués de grappes de personnes ou « groupe professionnel local » ayant des échanges réguliers sur des questions liées à leurs activités agricoles. L'introduction d'une nouvelle norme est mise en débat au sein du groupe qui, à travers un dialogue plus ou moins dense, la transforme, l'accepte, ou la rejette. Le groupe produit donc des connaissances pour savoir quoi faire et comment le faire. Mais tout le monde n'a pas la même position dans le groupe, certains y étant plus reconnus que d'autres. Ainsi tout argument est évalué par les membres du groupe selon deux dimensions : sa valeur propre correspondant à son intérêt pour l'action selon les normes en vigueur dans le groupe et sa valeur sociale correspondant à la place occupée par celui qui énonce l'argument.

Quand les collectifs d'acteurs ne sont pas homogènes ou structurés au sein d'une organisation, Akrich et al. (1988) montrent que l'innovation ne devient réalité que si elle est un enjeu pour les acteurs concernés et qu'ils peuvent l'intégrer dans leurs propres stratégies. L'innovation, c'est l'art d'intéresser un nombre croissant d'alliés qui renforce le processus. Son contenu mais aussi ses chances de succès résident entièrement dans le choix des représentants ou des porte-paroles qui vont interagir, négocier pour mettre en forme le projet et le transformer jusqu'à sa concrétisation. Cependant, l'innovation est loin d'être un processus linéaire et planifié mais s'apparente plus au modèle tourbillonnaire avec de multiples décideurs, de multiples petites et grandes décisions, beaucoup d'incertitude et beaucoup de désordre apparent. Olivier de Sardan (1998) insiste sur les porteurs sociaux qui occupent une place plus ou moins reconnue dans la société locale et par lesquels transite l'innovation. De même, l'innovation produit des effets directs et indirects, généralement différés, sur la structure sociale locale en servant certains intérêts et en contrariant d'autres : elle peut donc consolider la structure sociale en place ou au contraire favoriser des changements sociaux.

\section{Les systèmes d'innovations}

Pour rendre compte des réseaux qui naissent entre organisations, dépassant à la fois le modèle d'adoption linéaire et les réseaux informels, certains auteurs (Röling, 1990 ; Engel et Salomon, 1997) ont formalisé l'existence des « systèmes agricoles de connaissance et d'information » où les institutions de recherche et d'enseignement ainsi que les services de conseil agricole ne sont plus que des acteurs parmi d'autres dans les dynamiques d'innovation. La recherche prend ainsi place dans des systèmes d'innovation territoriaux ou sectoriels.

Le rôle des organisations dans les processus d'innovation a été d'abord formalisé dans le monde de l'industrie. Au sein d'un même territoire, les firmes et les services 
spécialisés peuvent former un cluster (Porter, 1998), qui constitue un ensemble social cohérent, partageant histoire et valeurs communes et reconnu pour sa dynamique d'innovation. À l'échelle d'un pays, les organisations de recherche et d'enseignement en charge de la production de connaissances ainsi que les acteurs industriels impliqués dans l'innovation peuvent former un système national d'innovation (Freeman, 1988 ; Lundvall, 1992). Dans ce système, les acteurs interagissent et créent de nouvelles connaissances, de nouveaux savoirs et savoir-faire. Les relations entre science et société s'en trouvent transformées. Selon Gibbons et son groupe de réflexion (1994), cette transformation est une rupture avec les séparations historiquement construites, qui faisaient de l'État, des entreprises et de la science, des sphères étanches. Nowotny et al. (2001) insistent également sur le fait que l'expertise est maintenant "socialement distribuée », ce qui conduit à considérer que chacun contribue à la connaissance, autrement dit que «nous sommes tous experts ». Cependant, cette lecture de la fusion des sphères de la connaissance n'est pas partagée par tous les analystes. Etzkowitz et Leydesdorff (2000) sont à l'origine d'un autre modèle d'analyse des relations science-société. Celui-ci est représenté par trois hélices représentant respectivement les sphères de l'administration publique, de la science et de l'industrie. Ce modèle prend en compte l'interaction entre ces sphères et leur co-évolution via les transformations institutionnelles, technologiques et scientifiques. Le développement de relations latérales entre sphères institutionnelles dessine alors de nouveaux espaces de savoirs, tout en permettant les transformations au sein de chaque sphère.

Le modèle de la triple hélice est toutefois critiqué par Shinn (2000) car il ne prend pas en compte l'existence d'une diversité de régimes de savoirs, c'est-à-dire d'une pluralité de modalités d'articulation entre acteurs scientifiques et socio-économiques. En effet, les organisations ne sont pas toutes égales et n'ont pas toutes les mêmes capacités d'innovation : celles qui ne sont pas capables d'adapter leurs routines aux nouvelles contraintes ou opportunités disparaissent comme le montre la théorie évolutionniste (Dosi et al., 2000). Cette approche permet de prendre en compte les trajectoires technologiques (Dosi, 1982) et l'évolution des régimes d'innovation. Les régimes dominants peuvent connaître des situations de blocage (lock-in) pour des raisons institutionnelles et des régimes alternatifs peuvent remplacer ou co-habiter, voire s'hybrider, avec un ancien régime dominant.

Les interrelations complexes entre acteurs, tant entre individus qu'entre organisations, ont amené à proposer le concept de «système d'innovations » pour mieux appréhender les dynamiques d'innovation. Il peut se définir " comme un réseau d'organisations, d'entreprises et d'individus produisant de nouveaux produits, de nouveaux processus, ou de nouvelles formes d'organisation mis en œuvre dans des activités économiques, et incluant les institutions et les politiques qui affectent leurs comportements et leurs performances » (Rajalahti et al., 2008).

L'approche en termes de systèmes d'innovation nécessite d'aborder différentes questions importantes. Les limites du système ne sont pas données mais sont construites : elles dépendent de la nature des questions posées mais aussi du point de vue des personnes qui les posent (Carlson, 2002). Ainsi, la littérature analyse des systèmes d'innovations locaux, sectoriels, nationaux, voire internationaux. Suivant les limites posées au système, l'identification des acteurs n'est pas toujours évidente, et la 
caractérisation de leurs ressources (matérielles et financières mais aussi en termes de connaissances, de compétences et de capacités) peut s'avérer difficile. Plus que les acteurs en présence, c'est la nature des interactions entre eux qui permet de caractériser le système d'innovation en précisant la nature des réseaux (formalisation, densité, flexibilité, etc.) afin d'accéder à des ressources, de générer des connaissances, de favoriser des processus d'apprentissage individuels et collectifs (Spielman et al., 2009). Dans ces réseaux, certains acteurs ont des positions privilégiées et jouent le rôle d'intermédiaire (Klerkx et Leeuwis, 2008). La nature des technologies qui est en jeu dans le système d'innovation (innovations incrémentales ou radicales, innovations « produit » ou de «processus ») a une influence sur la délimitation et la nature du système d'innovation. La gouvernance du système d'innovation renvoie aux relations entre acteurs et aux mécanismes formels ou informels mis en place pour programmer les actions, suivre et évaluer les résultats. Cette gouvernance dépend aussi du type d'innovations (tirées par le marché et des opérateurs de l'amont ou de l'aval de la production, promues par les producteurs ou les communautés pour saisir une opportunité ou répondre à une contrainte, facilitées par des entrepreneurs). Les politiques publiques peuvent favoriser les performances des systèmes d'innovation, en jouant notamment sur les dispositifs d'appui (plateforme, réseau, institution spécialisée) et de formation professionnelle, avec des financements articulant secteur privé et secteur public. L'évaluation des performances et des impacts des systèmes d'innovation pose la question des critères utilisés et donc des objectifs et des modèles visés.

\section{W Nouvelles questions dans un monde incertain}

Le bref historique sur l'évolution des travaux sur l'innovation montre un cheminement de la pensée scientifique. Aujourd'hui, le champ de la réflexion sur l'innovation relativise le poids des comportements individuels et s'élargit pour s'intéresser aux organisations (de conseil agricole, de recherche, de formation, voire de financement) et aux institutions qui définissent les règles du jeu entre ces acteurs. Cette approche en termes de systèmes d'innovation interroge donc le sens donné à l'innovation. Décrite dans les premiers travaux comme une nouvelle technologie, une nouvelle façon de produire ou une nouvelle façon de commercialiser (Schumpeter, 1934), elle devient le fruit d'interactions complexes, mobilisant des individus et des organisations, agissant dans des réseaux multiformes, permettant de créer des synergies ou révélant des oppositions. L'ensemble des travaux met alors en évidence la complexité et la non-linéarité de l'innovation (d'un concepteur vers un utilisateur ou d'un stade initial vers un stade final) et donc l'impossibilité de prévoir l'innovation, même si certains acteurs peuvent en stimuler le processus. Dans un contexte où les modèles de développement (y compris durable) sont fortement débattus et supposent des choix sociétaux importants, l'innovation apparaît entourée d'incertitude. Cette difficulté interroge la capacité des acteurs à innover pour concevoir de nouveaux systèmes de production et d'activités aptes à développer une agriculture durable dans différents contextes régionaux.

L'apparition du concept de systèmes d'innovation permet-elle de mieux mettre en débat différents modèles de développement ? Quels sont les défis actuels des 
systèmes d'innovation? La première partie nous invite à approfondir ces questions, à partir de trois points de vue.

Dans le chapitre 1, Lawrence Busch apporte les réflexions critiques de la sociologie américaine sur le rôle des normes de performance agricole dans l'orientation de la recherche. Celle-ci devient aujourd'hui un bien privé aux mains de grandes entreprises, figeant l'innovation agricole sur le terrain. Comment, dans un tel contexte, revenir aux enjeux planétaires : sécurité alimentaire, inégalités, changement climatique ? Il est temps d'inventer de nouvelles normes pour évaluer le résultat de la recherche et dépasser le critère unique de la productivité.

Rikka Rajalahti et Bernard Triomphe présentent dans le chapitre 2 la réflexion menée par la Banque mondiale sur les systèmes d'innovation. L'implication de nombreux acteurs au-delà de la recherche formelle oblige à s'interroger sur les défis en termes d'élaboration de politiques et de mécanismes institutionnels, pour permettre la mise en œuvre des systèmes d'innovation sur le terrain.

Le concept de système d'innovation permet certes de repenser la coordination de l'innovation, mais il présente parfois le risque de nous faire oublier la multitude d'expériences innovantes qui fourmillent hors du radar de la recherche. C'est le message que nous adressent Andy Hall et Kumuda Dorai (chapitre 3), en s'appuyant sur le réseau LINK (Learning INnovation Knowledge) qu'ils animent activement depuis quelques années. Ils montrent l'importance de comprendre qui sont les entrepreneurs innovants, souvent invisibles alors qu'ils sont à l'origine de nombreuses dynamiques de changement. Les auteurs suggèrent des pistes pour les encourager dans leurs démarches pionnières.

Après ces invitations à penser l'innovation autrement, la deuxième partie du livre aborde les questions que l'innovation pour le développement durable pose sur le terrain : comment concilier production agricole et préservation de l'environnement ? quels sont les effets d'exclusion de l'innovation ? Comment penser l'innovation dans la perspective d'un développement plus équitable ? Comment être créatif et construire de nouvelles connaissances utiles pour l'innovation ? Comment s'organiser collectivement, au niveau de filières ou de territoires, pour promouvoir l'innovation ? Les auteurs de chaque chapitre, chercheurs impliqués dans l'accompagnement d'innovation, ont synthétisé les apports originaux du symposium ISDA sur différents thèmes.

Jean-Marc Meynard s'intéresse dans le chapitre 4 aux effets du modèle agricole intensif, notamment de grandes cultures. Malgré ses impacts environnementaux négatifs, ce régime d'innovation se maintient par des effets de verrouillage (lock-in) dus à une trop grande spécialisation, les acteurs n'arrivant pas à redéployer leurs compétences vers d'autres alternatives de développement. Comment dès lors lever ces verrous? Il n'existe certainement pas « une bonne solution », mais l'auteur expose les principes d'une démarche « d'ingéniérie agro-écologique » qui permettrait aux acteurs eux-mêmes de se projeter dans l'avenir.

Denis Requier-Desjardins (chapitre 5) s'interroge sur la capacité de l'innovation à réduire la vulnérabilité de la frange la plus pauvre des populations rurales. En abordant la lutte contre la pauvreté en termes de développement des «capabilités » des acteurs, l'innovation agricole n'est plus une préoccupation aussi centrale 
qu'auparavant. La diversification des activités, agricoles ou non agricoles, l'immigration, l'insertion dans une économie sociale, sont autant de stratégies innovantes pour échapper à la pauvreté. Certes, de nouvelles politiques de lutte contre la pauvreté cherchent à renforcer le capital humain, mais elles encouragent encore rarement ces différentes formes d'innovation sociale.

Dans le chapitre 6, Estelle Bienabe, Johann Kirsten et Cerkia Bramley s'intéressent aux innovations tirées par les marchés agroalimentaires et la production de normes « durables ». Ils questionnent notamment les types d'action collective à l'origine de ces certifications, et leurs conséquences en termes d'équité. Tous les producteurs n'ont pas la même capacité à tirer profit des retombées des certifications, ni à maintenir leur participation à long terme dans les filières qui se mettent en place. Pour éviter l'exclusion des petits producteurs, une réelle construction sociale de la « qualité » est nécessaire, par la mise en place de processus d'empowerment, mais aussi par la définition de régulations publiques et privées, aptes à encadrer le développement de ces nouveaux standards.

André Torre et Frédéric Wallet (chapitre 7), responsables du programme de recherche « Pour et sur le développement régional $»^{3}$ en France, partent du constat que les études régionales ont souvent privilégié une conception des territoires comme «pôles de développement » construits autour d'innovations technologiques « high-tech ». Mais cette course à l'excellence technologique est tout à fait illusoire dans le cas de nombreux territoires ruraux. Quels sont alors les chemins de l'innovation pour ces territoires ? C'est au travers d'une gouvernance territoriale, vue comme espace de concertation mais aussi d'expression des oppositions, que pourront émerger et s'amplifier des processus d'innovation multi-niveaux, multi-acteurs et multi-facettes.

Ces réflexions venant du terrain montrent qu'il existe des expériences localisées intéressantes et porteuses d'un développement. Mais il est encore difficile de rendre opérationnel le concept de système d'innovation pour proposer, et surtout prioriser des politiques publiques capables de stimuler et renforcer les processus d'innovation à l'échelle d'un territoire ou d'un secteur économique. Pour nous éclairer sur ces implications en termes de politiques publiques, nous avons souhaité donner la parole à des représentants d'institutions, issus de différents continents, pour qu'ils partagent leur vision. Comment les décideurs politiques peuvent-ils promouvoir une nouvelle manière d'innover ? Comment institutionnaliser et consolider les expériences alternatives en véritable système d'innovation à large échelle ? Quels nouveaux outils politiques peuvent être développés?

Dans le chapitre 8, Juliana Santilli, procureur au ministère public du Brésil, expose à propos de la préservation de la biodiversité, comment les institutions internationales proposent des pistes d'innovation juridique que les États peuvent traduire en lois à leur niveau. Ainsi, le Brésil a promulgué des lois sur la protection de l'agrobiodiversité, en défiant les lois conventionnelles sur la propriété des semences qui ont longtemps bénéficié aux entreprises multinationales. Ces nouvelles lois garantissent

3. PSDR : Ce programme d'analyse et d'action sur le développement régional, à l'initiative de l'Inra, associe partenaires régionaux et recherche, dans 10 régions françaises, en lien direct avec les préoccupations des acteurs et avec un cofinancement égal entre les régions et les organismes de recherche. 
le droit des agriculteurs familiaux à utiliser et à multiplier leurs propres semences. La mise en ouvre sur le terrain en est à ses débuts mais cette affirmation légale constitue un premier pas pour imaginer une réorganisation du système semencier national.

Karim Hussein, de l'OCDE, et Khalid El Harizi, du FIDA, montrent dans le chapitre 9 que la conception de politiques pour l'innovation n'est pas suffisante, celles-ci doivent s'insérer dans un cadre institutionnel et politique plus large. Au travers d'exemples de politiques à différents niveaux, de l'international jusqu'au local, ils soulignent l'importance de développer les compétences des différents types d'acteurs et des plateformes d'échange pour concevoir des politiques plus flexibles, aptes à s'adapter à la diversité des demandes des acteurs de l'innovation. Suite au Printemps Arabe, ces considérations interpellent la capacité des gouvernements à permettre une réelle participation des acteurs locaux dans les processus d'innovation.

Dans le chapitre 10, Papa Seck, directeur d'Africa Rice, et ses co-auteurs Aliou Diagne et Ibrahima Bamba, nous invitent à redynamiser la gouvernance de la recherche agricole en Afrique. Pour eux, cela ne pourra se faire que si les chercheurs acceptent de s'engager dans des approches plus participatives. La co-construction, co-exécution et co-évaluation des politiques agricoles sont devenues indispensables à l'accompagnement d'une agriculture performante. Pour cela, un changement global, culturel et organisationnel est nécessaire.

Dans la conclusion, les questionnements abordés au cours du symposium ISDA sont mis en perspective. Même si de nouvelles manières de penser l'innovation se développent progressivement, même si des expériences intéressantes émergent, les modèles actuels de développement et les politiques de l'innovation apparaissent peu adaptés pour répondre aux immenses défis du monde agricole. Que faire pour repenser l'innovation dans le contexte actuel ? Comment déconstruire les modèles qui nous empêchent de penser différemment l'innovation? Comment explorer de nouvelles directions et construire de nouvelles connaissances dans un contexte incertain ? ISDA nous donne quelques éléments de réponse, mais ces questions restent ouvertes pour de prochaines rencontres.

\section{" Références bibliographiques}

Akrich M., Callon M., Latour B., 1988. A quoi tient le succès des innovations. Premier épisode : l'art de l'intéressement, Deuxième épisode : l'art de choisir les bons porte-parole. Gérer et comprendre. Annales des Mines, 11, 4-17 ; 12, 14-29.

Altieri M., 2002. Agroecology: the science of natural resource management for poor farmers in marginal environment. Agriculture, Ecosystems and Environment, 93, 1-24.

Argyris C., 1995. Savoir pour agir. Surmonter les obstacles à l'apprentissage organisationnel, Inter Editions, Paris.

Carlsson B., Jacobsson S., Holmén M., Rickne A., 2002. Innovation systems: analytical and methodological issues. Policy Research, 31, 233-245.

Coleman J., 1988. Social capital in the creation of human capital. American Journal of Sociology, 94, 95-120.

Chambers R., Pacey A., Thrupp L.A., 1989. Farmer first. Farmer innovation and agricultural research, Intermediate Technology Publication, London. 
Chauveau J.P., Cormier Salem M.C., Mollard E., 1999. L'innovation en agriculture, questions de méthodes et terrains d'observation, IRD.

Colonna P., Fournier S., Touzard J.M., 2011. Systèmes alimentaires, In : Pour une alimentation durable : réflexion stratégique duALIne (Esnouf C., Russel M., Bricas N., eds), Editions Quae, Versailles, 79-108.

Compagnone C., Auricoste C., Lémery B., 2009. Conseil et développement en agriculture : quelles nouvelles pratiques? Educagri - éditions Quae, Dijon, Paris.

Darré J.P., 1996. L'invention des pratiques dans l'agriculture, Paris, Karthala, 194 p.

De Schutter O., 2011. Rapport du Rapporteur spécial sur le droit à l'alimentation, Assemblée Générale des Nations Unies, 23 p.

Dosi G., 1982. Technological paradigms and technological trajectories: a suggested interpretation of the determinants and directions of technical change. Research Policy, 11, 147-162.

Dosi G., Nelson R.R., Winter S.G., 2000. The nature and dynamics of organizational capabilities, Oxford University Press, New York.

Etzkowitz H., Leydesdorff L., 2000. The dynamics of innovation: from national systems and "Mode 2" to a triple Helix of university-industry-government relations. Research policy, 29, 109-123.

Faure G., Gasselin P., Triomphe B., Temple L., 2010. Innover avec les acteurs du monde rural : la recherche-action en partenariat, éditions Quae - CTA, Presses agronomiques de Gembloux, 222 p.

Flichy P., 1995. L'innovation technique : récents développements en sciences sociales. Vers une nouvelle théorie de l'innovation, Paris, La Découverte, 255 p.

Freeman C., 1988. Japan: a new national system of innovation? Technical Change and Economic Theory, Pinter, London.

Engel P.G.H., Salomon M.L., 1997. Facilitating innovation for development. A RAAKS resource box. KIT Publishers.

Gibbons M., Limoges C., Nowotny H., Schwartzman S., Scott P., Trow M., 1994. The New Production of Knowledge, SAGE Publication, London, Thousand Oaks.

Jouve P., Mercoiret M.R., 1987. La recherche-développement : une démarche pour mettre les recherches sur les sytèmes de production au service du développement rural. Les Cahiers de Recherche-Développement, 16, 8-15.

Jouve P., 1994. Le diagnostic des conditions et modes d'exploitation agricoles du milieu. De la région à la parcelle, In : L'appui aux producteurs ruraux, Ministère de la Coopération-Karthala, Paris, 57-98.

Klerkx L., Leeuwis C., 2008. Matching demand and supply in the agricultural knowledge infrastructure: experiences with innovation intermediaries. Food Policy, 33, 260-76.

Lavigne Delville P., Sellamna N.E., Mathieu M., 2000. Les enquêtes participatives en débat. Ambitions, pratiques et enjeux, Collection Economie et Développement, éditions Karthala, Paris.

Lundvall B.A., 1992. National Systems of Innovation, Pinter, London.

Mendras H., Forsé M., 1983. Le changement social, Armand Colin, Paris.

Muchnik J., de Sainte-Marie C., 2010. Le temps des SYAL, Editions Quae, Versailles.

Norman D., Collinson M., 1985. Farming systems research in theory and practice, In : Agricultural Systems research for developing countries, Richmond, Australie, 12-15 mai 1985, ACIAR, No 11, 16-30.

Nowotny H., Scott P., Gibbons M., 2001. Re-thinking science: knowledge and the public in an age of uncertainty, Polity, Cambridge, UK.

Olivier de Sardan J.P., 1993. Une anthropologie de l'innovation est-elle possible, In : Séminaire d'Economie Rurale «Innovation et sociétés », 13-16 septembre 1993, Montpellier, 33-49.

Olivier de Sardan J.P., 1998. Anthropologie et développement. Essai en socio-anthropologie du changement social, APAD-Karthala, Paris, 221 p.

Perroux F., 1963. Introduction à l'économie du $x x^{e}$ siècle, PUF, Paris.

Porter M.E., 1990. The competitive advantage of nations, MacMillan, Basingstoke.

Rajalahti R., Janssen W., Pehu E., 2008. Agricultural Innovation Systems: from diagnostics toward operational practices, Discussion Paper 38, Washington, World Bank, 105 p.

Rogers E.M., 1983. Diffusion of innovations, third edition, Free Press, New York. 
Röling N., 1990. The Agricultural Research-Technology Transfer Interface: A Knowledge Systems Perspective, In : Making the Link; Agricultural Research and Technology Transfer in Developing Countries (Kaimowitz D., ed.), Westview Press/ ISNAR, pp. 1-4 et 11-23.

Röling N., 2009. Conceptual and methodological developments in innovation, In: Innovation Africa: enriching farmers' livelihoods (Sanginga P., Waters-Bayer A., Kaaria S., Njuki J., Wettasinha C., eds), Earthscan, London.

Rostow W.W., 1979. Stages of economic growth: a non-communist manifesto, Cambridge University Press, Cambridge, UK.

Sachs I., 1980. Stratégies de l'ecodeveloppement, Éditions Ouvières, Paris, 140 p.

Sanginga P., Waters-Bayer A., Kaaria S., Njuki J., Wettasinha C., 2009. Innovation Africa: enriching farmers' livelihoods, Earthscan, London.

Schumpeter, 1934. The theory of economic development: An inquiry into profits, capital, credit, interest, and the business cycle, Harvard University Press, Cambridge.

Scoones I., Thompson J., Chambers R., 1994. Beyond farmer first: rural people's knowledge, agricultural research and extension practice, Intermediate technology Publications, London.

Scoones I., Thompson J., 2009. Farmer First revisited, Practical Action Publishing, Rugby.

Sen A., 1989. Development as Capability Expansion. Journal of Development Planning, 19, 41-58.

Sen A., 1999. Development as Freedom, Oxford University Press, 343 p.

Shinn T., 2000. Axes thématiques et marchés de diffusion. La science en France, 1975-1999. Sociologie et sociétés, 1 (32), 43-69.

Spielman D., Ekboir J., Davis K., 2009. The art and science of innovation systems inquiry: Applications to Sub-Saharan African agriculture. Technology in Society, 13, 399-405.

Wenger E., 1998. Communities of practice. Learning, Meaning and Identity, Cambridge University Press, Cambridge.

World Bank, 2006. Enhancing Agricultural Innovation: How to Go Beyond the Strengthening of Research Systems, World Bank, Washington.

Yung J.M., Chauveau J.P., 1993. Débat introductif, In: Innovation et sociétés. Quelles agricultures? Quelles innovations ? actes du XIV séminaire d'économie rurale (Chauveau J.P., Yung J.M., eds), Montpellier, France, 17-32. 


\section{Partie 1 \\ Repenser l'innovation dans un monde incertain}





\title{
Chapitre 1 \\ Normes gouvernant l'innovation agricole \\ Lawrence Busch
}

\begin{abstract}
Dans nos recherches, nous accordons encore trop d'importance à l'objectif de croissance de la productivité comme mission première. Nous avons encore une foi trop grande en la toute-puissance de la connaissance des faits et des principes physiques et biologiques.

Nous devrions revenir avec un oeil critique sur nos politiques et nos programmes. Ces derniers sont-ils adaptés aux besoins de la nouvelle ère?
\end{abstract}

Kenyon Butterfield (1918 [1917], 54)

Ainsi que cette citation introductive le rappelle, Kenyon Butterfield, alors président du Massachussetts Agricultural College, il y a de cela un siècle, s'enquit de savoir si l'objectif de l'accroissement de la productivité était approprié à la « nouvelle ère ». Cette question est cependant restée essentiellement ignorée. Pour la majeure partie des trois siècles qui nous précèdent, la productivité a été l'objectif central et généralement incontesté de la recherche agricole. Une diversité de normes et de mesures associées ont été mises au point afin de définir la productivité. Comme toutes les normes, celles concernant la productivité règnent sur nos comportements, modèlent nos institutions et nos actions, et nous permettent de mesurer le "progrès ». Pourtant, nous sommes aujourd'hui à nouveau confrontés à la question posée par Butterfield. La productivité n'a pas perdu sa place et a peu de chances de la perdre mais elle s'est vue complétée à titre préliminaire par une série d'objectifs entremêlés et parfois litigieux se réclamant souvent de la «durabilité ».

Dans ce chapitre, je formulerai d'abord quelques observations concernant les normes. J'expliquerai ensuite les raisons pour lesquelles la productivité s'est trouvée intronisée en tant qu'objectif cardinal de la recherche agronomique, et comment les normes et les mesures ont été utilisées pour décrire et mettre en évidence les avancées en direction de cet objectif. Puis je montrerai pourquoi les défis auxquels l'humanité est actuellement confrontée nous obligent à :

- reconsidérer la pertinence de cet objectif;

- repenser les normes employées pour définir et mesurer ce dernier ;

- mettre au point de nouveaux objectifs, de nouvelles normes et de nouvelles mesures qui répondent à ces nouveaux défis.

En conclusion, je suggérerai comment nous pourrions aborder la difficile tâche d'agir de manière durable. 


\section{W Les normes : construire des réalités}

Que nous soyons scientifiques ou profanes, la plupart d'entre nous considérons les normes comme un sujet plutôt ennuyeux ; nous préférons nous consacrer à faire avancer les affaires en cours. Et pourtant, les normes sont précisément ce qui nous permet de le faire, en systématisant toutes sortes de processus, de produits et de pratiques (Bingen et Busch, 2005). Un sélectionneur souhaitant mettre au point un certain cultivar amélioré doit pouvoir disposer de normes (et des mesures associées) afin de savoir, par exemple, si la génération F1 constitue effectivement une amélioration. Un chercheur en protéomique ne peut contribuer à la connaissance des protéomes végétaux sans adopter les normes relatives à ce type de recherche (Evans, 2000). Un nutritionniste est incapable de calculer la valeur nutritive d'un aliment donné s'il ne peut étalonner ses divers instruments à l'aide d'un " matériel de référence » connu (National Institute of Standards and Technology, 2007). Et bien sûr, les poids et mesures standardisés sont indispensables à une recherche de qualité autant qu'au commerce international.

Mais les normes font plus qu'apporter des procédures, des règles, des exigences, des spécifications et autres choses connexes. Ce sont des dispositifs ontologiques. Ce sont les recettes à partir desquelles nous formons des réalités (Busch, 2011). Dis autrement, une fois qu'une norme a été promulguée et établie, si elle a tant soit peu de valeur, elle devient un acquis, une manière naturelle et " évidente » d'opérer. Elle tend à exclure les alternatives comme non pertinentes ou hors de propos, ou comme des pratiques médicales, productives ou scientifiques inappropriées ou inadéquates. En bref, elle nous permet de créer un ordre social et naturel toujours partial et incomplet, jusqu'à ce que son heure vienne d'être réajustée afin de prendre en compte de nouvelles politiques, de nouvelles inventions, de nouvelles pratiques ou de nouvelles découvertes. Ainsi, pendant plusieurs décennies, le « dogme central » a-t-il soutenu que seuls les gènes comptaient et que toute autre paire de base n'était que de l'ADN « de rembourrage », d'aucun intérêt scientifique ou social. Plus récemment, le dogme central a été remplacé par une nouvelle norme qui autorise le développement des sciences en «-omique» telles que la génomique, la protéomique, la métabolomique, et ainsi de suite.

Pendant une bonne partie des trois siècles qui nous précèdent, la productivité a été la « règle d'or » vénérée de la recherche agricole ${ }^{1}$. La déclaration récente et sans doute bien intentionnée du directeur de la FAO, Jacques Diouf, selon laquelle la productivité des cultures devrait être augmentée de $50 \%$ d'ici 2050 est ici paradigmatique. Jusqu'à récemment, cette norme est restée généralement incontestée, quoique les moyens employés pour la mesurer aient changé entre temps. Un bref aperçu récapitulatif des normes de productivité est esquissé dans les paragraphes suivants.

1. De même, durant l'essentiel du siècle passé, le produit national brut (PNB) a constitué l'étalon à l'aune duquel étaient mesurées les économies nationales. 


\section{W Avant la recherche agricole : l'exploitant expérimentateur}

La recherche en agronomie a débuté presque aussitôt que des hommes, que ce soit en réaction à la pression démographique ou par désir de sédentarité, se soient mis à cultiver la terre. Très rapidement, les fermiers ont commencé à sélectionner les semences et les plantes en recherchant l'hypertrophie des parties comestibles. Cette activité n'était certes pas un travail de recherche tel que nous le définirions aujourd'hui, mais elle correspondait à un type d'expérimentation par approximations successives. Elle a été à l'origine de la plupart des plantes que nous cultivons actuellement (Anderson, 1952, 1967). De fait, avant l'invention de la faux, de la faucille et des autres outils à moissonner, les grains mêmes étaient recueillis un à un lorsqu'ils parvenaient à maturité (Haudricourt et Hédrin, 1987). Cette opération permettait l'inspection attentive et le tri précis de chaque grain, les plus gros étant mis de côté pour les semailles de la saison suivante. Les normes, pour autant qu'elles existaient, étaient alors extrêmement localisées, variables dans le temps en fonction de la qualité de la récolte de l'année, rarement formalisées, et typiquement fixées par les décisions du fermier lui-même, ou éventuellement par la communauté agricole d'un même village. La durabilité était assurée en se conformant aux pratiques des ancêtres de chacun, en se pliant à des imitations souvent ritualisées de ce qu'avaient fait les membres de la génération précédente et en produisant des champs cultivés rappelant les paysages naturels (ou ce qui était alors défini comme tel) (King, 1911). Dans la plupart des cas, ce système fonctionnait, non pas tant parce les dieux avaient accueilli favorablement les rituels que parce que les transformations anthropiques du paysage et les réactions du monde naturel restaient encore relativement mineures.

Dans quelques cas, les pratiques agricoles ont effectivement entraîné un déclin à long terme plutôt qu'assuré une durabilité réelle, mais du fait de sa lenteur, cette évolution restait invisible aux yeux de ceux dont l'existence s'insérait à un moment donné en un lieu donné (Diamond, 2005). De manière générale, deux grands dangers menaçaient la durabilité : la baisse de fertilité des sols et la raréfaction des ressources en eau. Il est important de souligner que la fertilité des sols et les ressources en eau ont toujours été des phénomènes socio-naturels : toutes deux résultent à la fois de pratiques humaines et de processus naturels (Reboul, 1977). La baisse de fertilité se manifestait généralement lorsque des étendues relativement restreintes de terres étaient surexploitées pour nourrir une population croissante. Le manque d'eau se faisait habituellement sentir lors de modifications des caractéristiques climatiques - souvenons-nous de l'échec des toutes premières tentatives de colonisation dans la région des Grandes Plaines aux États-Unis - ou, plus brutalement, à l'occasion de l'effondrement de l'organisation sociale indispensable au maintien des canaux d'irrigation, comme en ancienne Mésopotamie. Ainsi que l'a exprimé l'historien William McNeill (1963, p. 32) : « de manière générale, plus les techniques d'acheminement de l'eau des Mésopotamiens devenaient sophistiquées, plus les tâches d'entretien devenaient lourdes et plus la probabilité d'une panne sporadique du système augmentait ».

Si le mode d'amélioration des plantes par tâtonnements utilisé par les fermiers était lent et malaisé, avec de nombreux échecs et retours en arrière, il a néanmoins permis 
la sélection et la modification de toutes les espèces végétales cultivées aujourd'hui. Les normes de productivité avaient un caractère très localisé, mais la plupart concernaient la taille des parties comestibles. Ce processus a fini par nous amener à une position de dépendance envers un nombre relativement limité d'espèces végétales (sans parler de leur dépendance envers nous-mêmes), les espèces moins productives étant abandonnées en faveur de celles dont les rendements étaient meilleurs. C'est ainsi que le riz est devenu la base du régime alimentaire dans la majeure partie de l'Asie tout comme le maïs dans ce qui est aujourd'hui appelé l'Amérique latine, et que le blé (ainsi que, dans une bien moindre mesure, le seigle et l'avoine) en est venu à remplacer le millet, l'épeautre et l'engrain en Europe. Bien plus tard, lorsque les colons européens ont commencé à emporter leurs plantes avec eux dans le monde entier, le blé est devenu la culture préférée dans une grande partie des plaines d'Amérique du Nord et du Sud. D'une manière semblable, dans les régions tropicales humides d'Afrique, le maïs a supplanté le sorgho comme céréale favorite. Sans ces efforts de nos ancêtres, la recherche plus formelle de ces derniers siècles n'aurait probablement pas pu produire grand chose.

\section{- La recherche agronomique en tant que bien public, 1600-1980}

\section{L'émergence du jardin botanique}

La première vague de ce qui serait aujourd'hui considéré comme de la recherche agronomique a commencé avec l'avènement du jardin botanique moderne. En Occident, les premiers de ces jardins ont vu le jour au cours du XVI ${ }^{\mathrm{e}}$ siècle $^{2}$. Leurs objectifs étaient multiples et interconnectés.

D'abord, ils devaient représenter une sorte d'encyclopédie vivante de la connaissance sur le monde végétal. Le jardin botanique idéal aurait ainsi accueilli au moins un spécimen de chaque espèce. Les plantes exotiques devaient être cultivées dans des serres où la température et l'humidité pouvaient être contrôlées. La plus connue de ces serres reste la Palm House du jardin botanique de Kew, dans laquelle des espèces de palmiers provenant de tout l'Empire Britannique étaient cultivées jusqu'à maturité. De telles collections vivantes ont beaucoup contribué à dynamiser l'étude de l'histoire naturelle et de la taxonomie des plantes, comblant les lacunes des codex botaniques des siècles précédents.

De plus, ils devaient permettre l'étude des plantes médicinales. En effet, bon nombre des premiers jardins étaient plus intéressants pour les espèces médicinales qu'ils contenaient que pour leurs objectifs agricoles. En France, celui connu sous le nom de Jardin royal des plantes médicinales, commandé par Louis XIII, n'a ainsi commencé à se développer au-delà du secteur médicinal qu'en 1718 (Bartélémy, 1979). On considérait à cette époque que toutes les espèces végétales avaient des

2. Des jardins de toutes sortes ont existé depuis les temps les plus reculés. Ce qui distingue ces nouveaux types de jardins est leurs liens avec les prémisses de la science moderne, elle-même inextricablement liée à la quête de colonies et à l'extension de la chrétienté. 
propriétés médicinales. Par ailleurs, en ces temps où les médicaments avaient autant de chances d'achever le malade que de le guérir, cultiver les plantes médicinales permettait au moins de garantir leur origine.

Les jardins botaniques allaient également permettre à l'espèce humaine de reprendre le contrôle de la nature en recréant le Jardin d'Eden. Les explorateurs portugais furent ainsi convaincus, pendant plus d'un siècle, que le Jardin du Paradis existait réellement et qu'il serait découvert au Brésil (Prest, 1981). Toutefois, comme il restait introuvable, il s'est agi par la suite de le re-créer en récoltant la totalité des espèces rencontrées dans une même localité. Ici, la promesse baconienne de « lire le livre de la nature » serait ainsi respectée ; l'œuvre de nature de Dieu serait lue tout autant que son œuvre écrite, la Bible.

Enfin, les jardins botaniques allaient permettre d'acclimater les plantes et de les cultiver dans d'autres contrées que celles dont elles étaient originaires, contribuant par là aux objectifs des puissances coloniales. Ainsi la Grande-Bretagne eut ses Kew Gardens (vers 1700), la France son Jardin des Plantes (1626), la Hollande son Hortus Botanicus (1638), l'Espagne son Real Jardín Botanico (1794) et ainsi de suite. Ce que suggère Calestous Juma (1989) concernant la Grande-Bretagne est tout aussi vrai pour les autres puissances coloniales : « d'une manière générale, l'Empire Britannique s'est étendu en grande partie grâce à l'application des connaissances botaniques, des évolutions technologiques et de l'organisation institutionnelle à la production agricole ».

Ainsi, pour résumer, les jardins botaniques ont repris à leur compte l'approche empirique mise en pratique par les agriculteurs depuis des millénaires et l'ont standardisée, systématisée et généralisée. Des expéditions à la recherche de nouvelles plantes étaient organisées jusqu'au bout du monde pour ramener des spécimens d'espèces potentiellement utiles pour les colonies. Les normes de productivité étaient ainsi centrées sur la capacité d'une espèce issue de la Colonie A à donner de gros rendements dans la Colonie $\mathrm{B}$, permettant ainsi de valoriser cette dernière. Il en a résulté que la production extrêmement lucrative de stimulants tels que le café, le thé, le cacao, le tabac et le sucre, et de matières premières industrielles telles que le caoutchouc, le jute et l'indigo, a été systématiquement étendue aux diverses colonies européennes partout sur le globe (Brockway, 1979).

\section{Des jardins aux stations expérimentales}

Les jardins botaniques se sont avérés relativement efficaces pour transférer des espèces d'un continent à un autre, mais leur approche empirique restait insuffisante pour ce qui était de l'amélioration de ces espèces à des fins agricoles. Ce ne fut qu'au XIX ${ }^{\mathrm{e}}$ siècle, avec l'entrée en scène d'un nouveau type d'institution, la station expérimentale, que l'essai en champ fut standardisé et institutionnalisé. La découverte de Justus Liebig, qui prit conscience que le développement des végétaux était directement lié aux minéraux du sol et qu'il était possible de produire des engrais, fut particulièrement importante. Le nombre des stations expérimentales explosa (une vingtaine avant 1851 à environ 1500 en 1930, d'après Busch et Sachs, 1981), avec, le plus souvent, le soutien enthousiaste de chacune des grandes puissances mondiales du moment. 
Avec le temps, les stations expérimentales ont apporté toute une gamme d'innovations au processus de recherche, en particulier :

- l'étude comparative de la performance (mesurée en unités standardisées du rendement en organes comestibles) de différentes variétés d'une même espèce cultivée ;

- l'utilisation de "groupes témoins » standardisés dans le cadre des comparaisons expérimentales ;

- l'inclusion progressive d'une multitude de variables (standardisées) dans les protocoles expérimentaux, dont les caractéristiques des sols, les besoins nutritifs des plantes, l'eau, la latitude, l'altitude, les pratiques culturales et ainsi de suite ;

- le recours à des méthodes statistiques standardisées pour comparer des résultats dans le temps et dans l'espace.

À part quelques exceptions, les stations expérimentales sont généralement restées, tout comme les jardins botaniques, directement rattachées à l'État ${ }^{3}$. Il y avait plusieurs raisons à cela. Tout d'abord, la plupart des fermiers ne pouvaient pas se permettre de financer le coût, relativement exorbitant, de fonctionnement d'une station expérimentale. Même ceux qui en avaient les moyens comprenaient rapidement qu'ils n'avaient pas la possibilité de monnayer à leur avantage les résultats de ces travaux de recherche, les voisins adoptant rapidement les innovations sans avoir contribué financièrement à leur mise au point. Enfin, et sans doute surtout, nourrir les centres urbains en plein développement et les classes laborieuses qu'ils hébergeaient était considéré, dans les pays "métropolitains », comme un facteur essentiel de la stabilité politique et de la solidité de l'empire colonial. Un enjeu à ce point déterminant ne pouvait être confié au hasard ; le contrôle de l'État était une condition nécessaire de la paix sociale.

Il en fut de même pour les centres internationaux de recherche agricole, instaurés au milieu du $\mathrm{Xx}^{\mathrm{e}}$ siècle sous la forme de projets inter-étatiques visant à répandre une révolution verte susceptible de faire obstacle aux révolutions rouges (voir par exemple Perkins, 1997). Ces centres ont, eux aussi, mis au point des produits dont des entrepreneurs privés n'auraient pas pu retirer un retour sur investissement. Ils étaient presque exclusivement consacrés à l'accroissement de la productivité, et mesuraient leurs avancées d'une manière pratiquement identique à celle des organismes de recherche agricole nationaux : en termes de rendement en organes comestibles par unité de surface.

\section{" La recherche agronomique en tant que bien privé, de 1980 environ à nos jours}

Au cours de la décennie 1980-1990, la recherche agronomique a amorcé une profonde transformation. Une série de modifications apparemment mineures mais décisives ont été introduites qui ont entraîné un glissement marqué du paysage de la recherche agricole.

3. Les exceptions étaient généralement centrées sur certaines espèces particulières cultivées en plantations (voir par exemple Grammer, 1947). 


\section{La propriété intellectuelle dans le monde végétal}

$\mathrm{Au} \mathrm{XIX}{ }^{\mathrm{e}}$ siècle, en dépit d'une petite exception pour certains micro-organismes, il était considéré dans le monde entier que les êtres vivants ne pouvaient faire l'objet de brevets. En 1930, les législateurs des États-Unis autorisèrent le brevetage de plantes. Des végétaux reproductibles par bouturage, essentiellement des variétés horticoles et fruitières, furent alors protégés en vertu des droits de la propriété intellectuelle. Dans la seconde partie du siècle, un système de protection des obtentions végétales fut instauré - une certaine forme de propriété intellectuelle qui permettait aux obtenteurs (en réalité, des semenciers privés) de protéger les nouvelles variétés qu'ils mettaient au point par sélection conventionnelle. Certaines voix s'élevèrent alors pour faire remarquer que cette évolution transformerait la recherche agricole. Selon l'un de ces observateurs, « si des considérations d'ordre international forcent les États-Unis à instaurer les droits des obtenteurs, il peut être escompté que les organismes publics se retireront progressivement des travaux d'amélioration de ces espèces cultivées pour recentrer leurs activités sur la recherche fondamentale » (Fejer, 1966).

Beaucoup plus récemment, dans le sillage de décisions de justice aux États-Unis, de nouveaux projets de droit positif dans l'Union européenne et de l'adoption par l'Organisation mondiale du commerce de l'accord sur les aspects des droits de propriété intellectuelle qui touchent au commerce (accord TRIPS), les plantes et les animaux sont devenus des cibles légitimes pour le dépôt de brevets industriels. En effet, la protection des obtentions végétales étant toujours en vigueur, la propriété est mieux défendue, dans un certain sens, du côté des plantes que du côté de l'industrie.

Toutefois, contrairement à ce qui se passe dans le cas de composants mécaniques ou même électroniques, pour lesquels il est possible et habituel de concevoir des moyens de contourner les brevets dans la mesure où, du moins en théorie, il existe un nombre infini ou très élevé de manières de venir à bout d'une même tâche, la nature même des êtres vivants est telle qu'un contournement de ce type est difficile, voire impossible. En outre, ainsi qu'une multitude de détracteurs l'ont fait remarquer, un droit de propriété sur un seul gène, tel que celui conférant la résistance au glyphosphate (Roundup ${ }^{\circledR}$ ), donne de fait au détenteur du brevet le contrôle de la plante en son entier.

\section{Les investissements privés dans la recherche sur les productions végétales}

En grande partie en raison de l'évolution du régime de la propriété intellectuelle (ou de son annonce), des entreprises privées ont commencé à investir de grosses sommes dans certains domaines de la recherche agronomique jusque là considérés sans grand intérêt. En effet, la quasi totalité des grandes sociétés agrochimiques, ainsi que bon nombre des sociétés pharmaceutiques et pétrolières, ont fait la démarche d'acquérir au moins une entreprise semencière, pensant que ce secteur pourrait compenser le déclin des bénéfices du secteur des produits chimiques en vrac et que s'en abstenir équivalait à se couper des nouvelles opportunités. Bien 
que les bénéfices extraordinaires promis ne se soient jamais matérialisés et que plusieurs compagnies pharmaceutiques et pétrolières aient fini par se défaire de leurs branches semencières et agricoles (par exemple, Novartis revendant sa branche agronomique sous le nom de Syngenta), un petit nombre de sociétés d'envergure comparativement plus importante ont maintenu leur engagement dans la recherche sur les productions végétales.

Au demeurant, selon un rapport du département de l'Agriculture des États-Unis : «Le secteur privé a investi au moins 3,4 milliards de dollars dans la recherche agroalimentaire en 1992, tandis que le secteur public investissait 2,9 milliards. Plus de $40 \%$ des activités de recherche et développement agricoles privées sont consacrées à la mise au point de nouveaux produits, contre moins de $7 \%$ dans la recherche agronomique publique » (Fuglie et al., 1996).

En s'appuyant sur des données mondiales se rapportant aux années 2000, Pardey et al. (2006) font remarquer que le secteur privé a couvert $36 \%$ de l'ensemble des dépenses liées à la recherche dans le monde, et $54 \%$ de ces dépenses dans les seuls pays à haut revenu ${ }^{4}$. Étant donné que l'essentiel de la recherche agronomique est réalisé dans ces derniers, le second chiffre reflète sans doute mieux la dominance actuelle du secteur privé dans la recherche agricole. Cette dominance signifie, entre autres choses, que, pour le meilleur et pour le pire, l'essentiel de l'ordre du jour dans ce domaine est dicté par le secteur privé.

\section{Encadré 1.1. Semences : du bien marchand au bien commun Pieter Lemmens et Guido Ruivenkamp}

Depuis les débuts de l'agriculture, les semences ont été considérées comme un bien commun et étaient échangées librement entre agriculteurs, partout dans le monde. L'innovation agricole a ainsi toujours été fondée sur la coopération et le partage. Cette situation a changé du tout au tout au fil des deux derniers siècles avec la mainmise progressive des institutions publiques sur l'innovation, puis, au cours des trois dernières décennies, avec celle sans cesse renforcée des entreprises privées. Aujourd'hui, de plus en plus, le public et le privé se rejoignent pour consacrer leurs efforts de recherche combinés à la mise au point de variétés parfaitement adaptées à une agriculture devenue industrielle et intégrée dans des chaînes alimentaires mondialisées. De biens communs, les semences ont été transformées en biens marchands, produits pour le profit et conçus pour amener la production agricole sous l'emprise des sociétés agroalimentaires.

La prise en main de l'innovation agricole par le secteur public, et a fortiori le privé, a entraîné, ainsi que beaucoup l'ont souligné, un processus de prolétarisation chez les utilisateurs (agriculteurs et producteurs) au sens d'une expropriation de leur savoir et de leur savoir-faire, d'une perte de leur capacité et d'un désengagement de leur responsabilité dans la sélection et la culture des plantes. Ces prérogatives ont maintenant été déléguées aux scientifiques, dont le programme de recherche est de plus en plus soumis au capital d'entreprise. Le capital, par sa nature même, est incapable de faire preuve d'intérêt altruiste et de responsabilité puisque sa préoccupation

4. En France, au Royaume-Uni et aux États-Unis, le secteur privé a couvert respectivement 75,72 et $52 \%$ du total des dépenses de recherche depuis 2000. 
principale est de faire des profits et qu'il est structurellement indifférent aux valeurs sociales et écologiques.

Afin de redonner à l'agriculture le sens du soin et de la responsabilité, et de la rendre plus durable et plus équitable, il importe de raviver non pas tant le secteur public (dont la dépendance envers les logiques du privé va croissant) que l'esprit des biens communs, en trouvant des moyens de replacer l'innovation dans la sphère des biens communs et en contrant les processus de prolétarisation et de marchandisation des semences et du matériel génétique par des processus de mise en commun. Des initiatives en open source, telles que l'organisation Cambia-BiOS qui propose une approche de l'innovation agricole fondée sur l'idée de bien commun, constituent un pas important dans cette direction et pourraient être de plus en plus adoptées et élaborées par des paysans, des organisations de la société civile, des exploitants agricoles et des scientifiques.

Le principe directeur de l'innovation open source réside dans la transparence et la libre accessibilité des outils de l'innovation. Son orientation première n'est pas la création d'une valeur d'échange - le profit - mais d'une valeur d'usage, à savoir l'expansion perpétuelle d'un fonds de ressources commun. Cambia est fermement convaincu que l'innovation est à son optimum d'efficacité et de productivité non pas lorsque les outils et l'information sont privatisés et convertis en propriété exclusive, mais lorsqu'ils sont mis en commun et rendus non-exclusifs. Sa mission est de démocratiser l'innovation, et à cette fin cette organisation a mis au point une série d'outils - à l'instar de sa technique employant des vecteurs TransBacter en alternative au vecteur breveté Agrobacterium - et de pratiques de recherche rendus accessibles aux termes d'une licence libre inspirée de la licence GPL Free Software inventée par Richard Stallman dans le cadre de l'industrie du logiciel. Par le biais de cette licence, Cambia vise à créer un fonds commun protégé de techniques et de capacités à l'abri de toute appropriation par des organismes privés. Elle s'engage ainsi clairement en faveur du renforcement du pouvoir décisionnel des utilisateurs, plutôt que des propriétaires (Lemmens, 2010 ; Ruivenkamp et Jongerden, 2010).

\section{Les mutations de la recherche publique}

Une série de glissements décisifs a affecté la recherche publique : le passage des dotations globales récurrentes aux allocations concurrentielles, la modification subtile mais profonde de la manière d'évaluer et de récompenser les chercheurs, une évolution des compétences de ces derniers, le retrait progressif du soutien aux activités de vulgarisation, l'apport croissant de fonds privés dans les organismes publics et le quasi effondrement des financements publics dans certains pays. Examinons tour à tour chacun de ces points.

L'octroi de dotations globales aux institutions était une pratique courante depuis la création des stations expérimentales au $\mathrm{XIX}^{\mathrm{e}}$ siècle. Un organisme de recherche ou d'enseignement supérieur recevait chaque année de l'État une enveloppe qu'il utilisait pour poursuivre ses travaux de recherche en agriculture. La répartition de ces crédits était généralement décidée par le directeur de l'organisme concerné, secondé à des degrés divers par les chercheurs employés et parfois par les exploitants utilisateurs des produits. Ce mécanisme de financement se justifiait à plusieurs titres, et notamment par le fait que le travail expérimental exigeait des infrastructures 
relativement lourdes. Ainsi les essais mettant en jeu des bovins ou d'autres espèces animales demandaient-ils d'entretenir un troupeau entier pour une période indéfinie ; de même, les expériences sur les végétaux devaient pouvoir disposer de vastes étendues de terres arables pour la conduite des essais en champs. Qui plus est, la gestion de ces activités était, et demeure à ce jour, un exercice complexe, réclamant des efforts considérables pour veiller à ce que les traitements appliqués dans le cadre d'une expérience n'influencent pas les résultats des expériences qui suivent au même endroit. Cependant, ces dernières années, ce type de financement est tombé en désuétude. Ce déclin a plusieurs causes, dont l'obsession néolibérale d'introduire de la concurrence dans toutes les activités (Bourdieu, 1998) - une obsession qui a commencé depuis peu à s'imposer dans le monde entier. Étant donné que la plupart des financements attribués dans un cadre concurrentiel couvrent rarement plus de 3 à 5 ans de recherche, les travaux à long terme en ont souffert. De plus, il existe au moins une étude récente suggérant que cette évolution a directement entraîné une diminution des gains de productivité des cultures (Huffman et Evenson, 2006).

Si les résultats de la quasi totalité des travaux de recherche en agronomie étaient, il y a un siècle, publiés dans des bulletins - en général des publications internes d'épaisseur variable, ils parvenaient pour l'essentiel, 50 ans plus tard, à se frayer un chemin

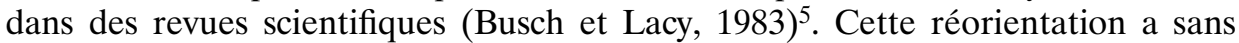
doute été en partie suscitée par la professionnalisation croissante des chercheurs, ainsi que par la prise de conscience des larges répercussions que pouvaient avoir les résultats de la recherche agricole ${ }^{6}$. Au cours des dernières années, les mécanismes d'évaluation et de promotion en vigueur dans les institutions de recherche publiques ont modifié leur angle d'approche et accordent plus d'importance au nombre d'articles publiés dans des revues scientifiques (souvent par opposition à l'obtention de « produits », tels que des nouvelles variétés de plantes, des meilleures pratiques culturales, ou des innovations dans le mode de conduite des cultures ou des troupeaux) et, dans certains organismes, au nombre de citations de ces articles. Il en résulte que les travaux conventionnels d'amélioration d'espèces animales et végétales, ainsi que toutes les activités scientifiques dont les résultats ne s'obtiennent pas à court terme, ont été dévalorisés, voire abandonnés. En effet, dans les organismes qui privilégient l'analyse des citations, les chercheurs subissent une pression accrue pour publier leurs articles dans les revues les plus citées et leurs ouvrages chez les éditeurs les plus prestigieux. Ce changement dans le système d'évaluation présente de grands avantages pour les administrateurs de la recherche. En particulier, il semble produire un moyen apparemment objectif de comparer les résultats obtenus par différents chercheurs œuvrant dans des domaines scientifiques distincts sur des sujets d'importance diverse. Il semble fournir une mesure du succès d'un chercheur donné au sein du « grand marché des idées ». Toutefois, cette manière de voir comporte plusieurs écueils de taille. Il est certes aisé de compter des articles, mais dans beaucoup de

5. Au début du $\mathrm{xx}^{\mathrm{e}}$ siècle, déjà, les directeurs des stations expérimentales des États-Unis s'inquiétaient du fait que les bulletins ne constituaient pas le meilleur support pour la publication des résultats de la recherche (voir par exemple Pearl, 1915).

6. Pour le formuler d'une manière plus simple, les résultats obtenus en une localité sont presque toujours applicables ailleurs. Ils ont des répercussions dans d'autres espaces géographiques qui présentent des caractéristiques environnementales voisines. 
disciplines, il est tout à fait possible à un chercheur de fractionner son travail en tranches toujours plus spécialisées, multipliant ainsi artificiellement le nombre d'articles publiés. De plus, le rythme de production des articles est étroitement lié au type de recherche effectuée : si certaines expériences peuvent être complétées en un mois, d'autres s'étendent sur plusieurs années. Des problèmes semblables sont à relever en ce qui concerne l'utilisation des citations. Tout d'abord, les bases de données de citations ne sont ni complètes ni aléatoires; elles ne prennent en compte qu'un certain nombre de revues - excluant bien souvent celles qui ne sont pas en langue anglaise, ou qui concernent des domaines très spécialisés ou très récents. De plus, les taux de citation dépendent étroitement du nombre de personnes qui travaillent dans le domaine en question; quelle que soit l'importance réelle de leurs travaux, les chercheurs qui œuvrent dans des secteurs peu étudiés ne peuvent être cités autant que ceux dont le domaine est très couru. Certains articles sont cités pour les erreurs qu'ils contiennent plutôt que pour leurs apports : l'article de Hwang Woo-Suk consacré aux cellules souches et publié par la revue Science a ainsi été cité 198 fois selon le Science Citation Index alors qu'il s'est avéré frauduleux. Ensuite, il existe au moins quelques cas avérés d'éditeurs ayant encouragé les auteurs à multiplier les références dans leurs articles afin d'accroître le taux de citation (et sans doute rehausser par là le statut) de certaines revues. Enfin, il arrive que la valeur de travaux de recherche ne soit reconnue par la communauté scientifique qu'après un certain délai ; en cela, les récapitulations annuelles des citations peuvent ne rendre qu'une image contrefaite de la réalité. Toutefois, l'aspect le plus pervers de cet usage des citations est qu'il sape la recherche scientifique dans son élan même. Il encourage les meilleurs chercheurs à travailler sur des thèmes reconnus, intéressant de vastes zones géographiques, et qui n'ont bien souvent qu'un lien distant avec les problèmes auxquels se heurtent les agriculteurs sur le terrain (voir par exemple Hanafi, 2010). Qui plus est, les sujets trop audacieux sont évités. Après tout, les recherches à haut risque sont, par définition, plus exposées aux échecs et de ce fait plus susceptibles de donner des résultats impubliables, ou seulement acceptables dans des revues peu connues ${ }^{7}$.

La conjonction des modifications du système d'évaluation et de la percée du nouveau secteur florissant de la biologie moléculaire végétale a conduit les directeurs de recherche à revoir leurs politiques de recrutement. Auparavant les instituts de recherche agronomique s'assuraient chacun les services de quelques taxonomistes et d'un grand nombre de sélectionneurs, désormais ces spécialités sont considérées comme dépassées. Le jour où l'éminent taxonomiste Jack Harlan (1936), qui avait tiré la sonnette d'alarme dès les années 1930 au sujet du déclin des ressources génétiques, a pris sa retraite de l'Université de l'Illinois, il n'a pas été remplacé. Le manque de sélectionneurs de végétaux commençait ainsi déjà à se faire sentir dans les années 1990 (Frey, 1996). Les biologistes moléculaires, cependant, quelle que soit la qualité de leur travail, n'ont rien à offrir directement aux agriculteurs.

7. Le cas du prix Nobel d'économie George A. Akerlof est ici instructif. Son article désormais célèbre, intitulé The Market for 'Lemons' (Akerlof, 1970) a été rejeté par les revues de premier rang de son domaine parce qu'il remettait en cause le statu quo en vigueur (Cassidy, 2009). S'il s'était trouvé dans le cadre du système actuel d'évaluation des chercheurs, il aurait pu être complètement découragé de publier ce travail. 
Pendant ce temps, les activités de vulgarisation et de conseil agricole sont allées à vau-l'eau, en particulier dans les pays pauvres, mais également dans certains pays riches, tels que les États-Unis. Pour les administrateurs et les politiciens, cette évolution était dans l'ordre des choses puisque la recherche publique générait moins de produits directement utilisables par les agriculteurs, la plupart de ces produits nécessitant une période de « développement » dans le cadre d'entreprises privées. Pendant ce temps, le secteur privé a accru ses propres efforts de "vulgarisation » alors qu'augmentait son potentiel de ventes. Par ailleurs, dans les pays riches, avec la diminution du nombre d'agriculteurs, les activités publiques de vulgarisation et de conseil voyaient leur justification fragilisée (Wolf et Zilberman, 2001). C'est ainsi qu'en Nouvelle Zélande le service de conseil agricole en son entier a été privatisé, avec instauration d'un régime de rémunération à l'acte.

La gêne qui était auparavant ressentie envers l'acceptation de fonds privés pour la recherche ou envers la participation à des projets mixtes publics-privés s'est évaporée ${ }^{8}$. Les universités et les laboratoires publics ont commencé à considérer les capitaux privés comme une nouvelle source de revenus, susceptible de remplacer les fonds publics « inadéquats » ou en baisse. De fait, l'investissement privé dans les institutions de recherche publiques est passé du goutte à goutte à l'inondation. Aux États-Unis, en 1994, environ $20 \%$ des recherches conduites sur les stations expérimentales agricoles d'état étaient financées par le secteur privé, y compris lorsque les dépenses publiques par les gouvernements fédéral et local stagnaient ou déclinaient. L'accord désormais plutôt tristement célèbre entre l'Université de Californie Berkeley et la société Novartis constitue un cas paradigmatique de ces types de relations (Rudy et al., 2007).

Certains pays, enfin, ont été le théâtre d'un abandon partiel ou total de la recherche publique agricole. C'est le cas du Royaume-Uni, où le prestigieux Cambridge Plant Breeding Institute a été vendu au secteur privé et où quantité d'autres organismes de recherche agronomique ont été fermés. Ailleurs, la recherche publique est bien souvent en état de stagnation ou de délaissement quasi absolu (cf. par exemple Echeverria, 1998).

\section{" Les supermarchés, les transformateurs et la révolution des normes}

Mais l'histoire ne s'arrête pas là. La dernière décennie a été le témoin du développement phénoménal et en grande partie imprévu du champ d'action et de la puissance des acteurs avals des chaînes d'approvisionnement alimentaires. Notamment, un petit nombre de grandes chaînes de supermarchés bien organisées ont pénétré des marchés considérés jusque là comme trop étroits, trop pauvres et trop désorganisés pour être intéressants (Dries et al., 2004 ; Reardon, et al., 2003 ; Weatherspoon et Reardon, 2003). Cette mutation a mis sur le devant de la scène des thèmes relevant

8. Un rapport américain de 1928 soutenait fermement que les capitaux privés ne devaient être acceptés que pour des travaux de recherche d'importance publique générale, et encore, seulement si ces crédits étaient octroyés à l'institution plutôt qu'à un chercheur particulier (Barre et al., 1928). 
de l'aval de la filière y compris les questions d'alimentation, de qualité des aliments et de durabilité tout en apportant dans son sillage un certain nombre de problèmes sérieux. En particulier, dans leur zèle à protéger et préserver leurs chaînes d'approvisionnement (Busch, 2007) et à éviter les risques qu'impliquerait, pour leur réputation, le fait de vendre des produits contaminés, les grandes surfaces ont commencé à imposer une multitude de normes à leurs fournisseurs (Fulponi, 2006; Mutersbaugh, 2005). Les agriculteurs et les entreprises de transformation se doivent ainsi d'être efficaces et productifs de manière générale, mais également dans certains domaines hautement spécifiques. Une vaste gamme de certifications et d'audits a de ce fait été commandée par les détaillants. Bien que ces normes, certifications et audits soient en principe facultatifs, ils sont, dans la pratique, obligatoires. Tout défaut de respect étant puni de l'impossibilité d'écouler sa production.

Ces myriades de normes ont pour résultat de geler l'innovation sur les exploitations. Additionnées les unes aux autres, et certainement sans malice de la part de la grande majorité des chaînes de supermarchés, elles constituent un fardeau considérable pour les fournisseurs. Ces derniers sont tenus d'utiliser certains cultivars bien particuliers, de répondre à des critères cosmétiques stricts de qualité, d'utiliser des pratiques culturales et des modalités d'application d'engrais et de pesticides définies à l'avance, de récolter et de livrer à des dates prédéterminées, et de conditionner les produits dans des contenants de tailles spécifiées. Toutes ensemble, ces normes ont pour effet - généralement non intentionnel mais néanmoins pernicieux - de canaliser les efforts de recherche dans certaines directions. N'étant pas intégrées à une vision d'un régime plus durable de production et de distribution alimentaires, elles sont susceptibles de faire obstacle aux avancées en matière de durabilité.

Pour résumer, en raison de la diminution de la part publique, voire dans certains cas de l'instabilité ou de la baisse des financements en général, du passage des dotations globales récurrentes aux allocations concurrentielles dans les mécanismes de financement, des changements intervenus dans le système d'évaluation et de récompense des chercheurs qui détournent ces derniers des travaux menant directement à des applications pratiques, du retrait progressif du soutien public aux activités de vulgarisation et de conseil agricole, et de la montée en puissance des chaînes d'approvisionnement, détaillants à leur tête, le secteur privé est devenu, lentement mais sûrement, l'élément directeur central de la recherche agronomique. Autrement dit, la recherche est devenue en grande partie un bien privé. Les organismes publics eux-mêmes tendent à récompenser les recherches qui parviennent à capter de nouvelles sources de revenu - que ce soit par le dépôt de brevets ou par la création d'entreprises exploitant directement les résultats de ces travaux plutôt que la recherche d'intérêt général. De plus, le renforcement des droits de la propriété intellectuelle et de la règlementation connexe, d'une part, et la position dominante du secteur privé, d'autre part, suscitent ensemble une diminution de l'ampleur des retombées classiques de la recherche agronomique. En d'autres termes, si autrefois le produit des recherches était souvent facilement adaptable à différents environnements, il n'en va plus de même de nos jours. Ainsi que l'expliquent Pardey et al. (2006) : « En particulier, les pays riches sont en train de réorienter leurs activités de recherche et de développement agricoles vers d'autres technologies que celles qui sont le plus facilement adaptées et adoptées par les pays en voie de développement. De plus, les droits de la propriété intellectuelle, ainsi que d'autres politiques de 
réglementation dont les protocoles en matière de biosécurité, les régimes commerciaux et les restrictions réglementaires spécifiques sur la circulation du matériel génétique influencent de plus en plus le degré auquel de telles retombées sont envisageables ou économiquement rentables ».

\section{Wa tempête qui se profile}

Ce que je viens de décrire n'aurait pas de grandes conséquences si les normes et les mesures mêmes qui sont utilisées pour définir et calculer les gains de productivité ne nous égaraient. Actuellement la communauté scientifique se trouve confrontée, comme chacun sur cette planète, aux problèmes suivants :

- environ un cinquième de la population mondiale est sous-alimentée malgré l'accroissement considérable de la productivité ;

- nous sommes plus que jamais dépendants des énergies fossiles non renouvelables pour maintenir les niveaux de productivité - non seulement sur les exploitations, mais également dans la fabrication des intrants nécessaires et dans la distribution de la production par la suite ;

- le macro-élément limitant est le phosphate ; les possibilités d'approvisionnement en sont relativement limitées tandis que l'efficacité de son utilisation reste faible ;

- l'obésité est un problème significatif qui gagne du terrain, et ce, non seulement dans les pays développés, mais encore dans les pays à revenu intermédiaire et dans les pays pauvres ;

- les méthodes actuelles de l'industrie agroalimentaire sont la cause de nombreux problèmes environnementaux, dont la contamination des eaux de ruissellement par les nitrates, l'érosion et l'appauvrissement des sols, et la diminution de la biodiversité ;

- le changement climatique aura probablement pour effet de modifier les conditions de la production agricole dans beaucoup de régions, entraînant une perte de cohérence entre les ressources locales en eau et les plantes cultivées ;

- les termes des échanges entre les pays industrialisés et les pays pauvres continuent à se détériorer.

Chacune de ces questions constitue à elle seule un obstacle à la mise en place d'un approvisionnement alimentaire durable. Mais, d'une manière tout aussi importante, les normes et les mesures employées actuellement en matière de productivité tendent à masquerces problèmes déconcertants. Elles perpétuent en effet deux mythes : l'accroissement de la productivité permettra à elle seule de résoudre les problèmes d'alimentation et d'agriculture ; il n'existe qu'une seule voie vers le développement durable, que tout individu et toute nation se doit d'emprunter. Ce sont là des mythes, certainement, mais des mythes réels, dans la mesure où, ainsi que l'a suggéré W.I. Thomas (1928), ils sont « réels quant à leurs conséquences ». Au mieux, ils transmettent des signaux erronés aux chercheurs et aux autres acteurs de la filière agroalimentaire ; au pire, ils cachent les graves vulnérabilités et occultent les obstacles qui pavent le chemin vers une manière plus durable de nourrir l'humanité (Tansey et Rajotte, 2008 ; Weis, 2007). 


\section{Encadré 1.2. Nouvelles normes d'évaluation de la recherche : le cas du système de riziculture intensive \\ Shambu Prasad Chebrolu}

La première décennie du $\mathrm{XxI}^{\mathrm{e}}$ siècle a été le témoin d'une vague de désespoir sans précédent dans le monde agricole qui a poussé au suicide plus de 200000 cultivateurs indiens, la plupart de régions qui avaient auparavant été concernées par la révolution verte. Cette situation appelle à un ré-examen du paradigme de la science agronomique qui a privilégié l'accroissement de la productivité par rapport à la réduction de la pauvreté et à la durabilité écologique. De manière surprenante, la même période a également vu l'expansion progressive et régulière de pratiques agroécologiques et d'innovations telles que le système de riziculture intensive (SRI). Le SRI consiste en un ensemble d'idées et d'intuitions qui ont vu le jour à Madagascar à la suite des expériences méthodiques d'un religieux français. Six principes de base ont ainsi été dégagés qui permettent ensemble de créer un milieu plus favorable au développement du riz, à savoir un plus grand espacement des plants, le repiquage brin par brin, le maintien des parcelles humides mais non inondées, l'aération du sol par un désherbage mécanique, et l'utilisation d'un amendement organique. Ce système de culture accroît les rendements et la santé des sols et réduit les besoins en intrants (semences, eau et travail) sans changer ou améliorer les variétés utilisées.

Le SRI a reçu un accueil froid, voire hostile, dans les cercles officiels de la recherche sur le riz. Une fixation sur les niveaux de productivité à la ferme et une polémique à propos de rendements déclarés excédant le maximum biologique de l'espèce ont eu pour conséquence que le SRI n'a pas été considéré comme une alternative sérieuse en matière de riziculture. Ses défenseurs ont attiré l'attention sur l'effet plus général de ce système, qui permet non seulement d'augmenter les rendements et la productivité, mais encore d'améliorer le revenu des exploitations (en particulier des paysans pauvres), la préservation des ressources (notamment du fait des besoins moindres en eau), la résistance aux ravageurs et aux maladies, ainsi que la résilience face aux aléas climatiques que sont les sécheresses, les tempêtes et les extrêmes de température. Il est estimé qu'entre 2 et 3 millions d'agriculteurs ont adopté le SRI dans le monde. En raison des controverses qui existent concernant son rendement potentiel, de l'impossibilité de faire des réplicats et d'estimer les effets de synergie en conditions de laboratoire souvent sur des sols dépourvus de toute activité microbienne, les scientifiques ont omis de prêter attention aux expériences conduites par les exploitants eux-mêmes et qui ont été à l'origine de la diffusion rapide de cette technique dans les campagnes. Les investissements publics dans les activités de recherche et de conseil agricole en matière de SRI ont été limités et ont bénéficié de peu d'apports privés. Il reste que le SRI, évoluant dans le domaine public, en accès libre, offre un modèle alternatif d'architecture des connaissances et permet le renouveau de l'esprit des biens communs en agriculture.

Les quelques chercheurs qui ont étudié le SRI ont souligné la possibilité que la physiologie du plant soit modifiée dans ces conditions particulières et ont insisté sur la nécessité de mettre en place de nouvelles normes pour pouvoir évaluer ce système. La diffusion du SRI s'est opérée en grande partie grâce à Internet, qui a rendu possible un nouveau type de recherche publique avec libre échange des informations et des idées entre chercheurs, agriculteurs, donateurs et organisations de la société civile. Des groupes créés sur la toile, comme le googlegroup SRI-India, qui compte plus de 400 membres, et d'autres plus récents tels que les « alliances d'apprentissage » de coalitions pluri-acteurs, ont eu un rôle moteur dans cette propagation. 
Les principes du SRI ont par ailleurs été étendus à d'autres espèces cultivées. Ce qui se passe sur le terrain souligne la nécessité de ré-examiner attentivement le fait que les normes telles que la productivité et le rendement soient les seuls critères utilisés pour définir la croissance agricole, et de faire pression pour que soit adoptée une définition pluraliste qui prendrait en compte le revenu de l'exploitation, la santé des sols et les stratégies de préservation des ressources (Chebrolu et Sen, 2010).

Pour mieux percevoir l'importance des problèmes qui se profilent, tentons ensemble un petit exercice de réflexion. Imaginons que, grâce à un extraordinaire exploit scientifique, la productivité des principales cultures à partir de l'année prochaine puisse doubler. En d'autres termes, supposons qu'il soit possible de produire deux fois plus de denrées avec la même quantité d'intrants. Cela ne se traduirait pas par une division par deux du prix des denrées pour les consommateurs, puisque 20 à $25 \%$ seulement du coût des aliments est lié aux activités des exploitations agricoles. Le prix de la nourriture ne baisserait que d'environ 10 à $15 \%$. Il s'agirait certainement d'une amélioration significative de la situation pour ceux qui se trouvent au bas de l'échelle des revenus, mais l'immense majorité de ceux qui ont faim auront encore faim. En outre, tous les autres problèmes associés aux conditions de la production des denrées alimentaires existeraient toujours : raréfaction des ressources, pollution de l'environnement, obésité, etc.

\section{" Élargir le champ d'investigation de la recherche agronomique}

Tout ce qui précède suggère qu'il existe une besoin impérieux d'élargir le champ d'investigation des recherches en matière d'agriculture. La productivité ne pourra plus constituer qu'un objectif parmi d'autres. Des normes et des mesures devront être mises au point pour s'attaquer au problème pernicieux (wicked problem) de la durabilité (Batie, 2008 ; Rittel, 1972 ; Rittel et Webber, 1973). En effet, il n'y a pas une solution optimale mais plutôt des équilibres à trouver entre une multiplicité d'objectifs. Les paramètres ne sont pas tous connus ou ne peuvent pas tous être spécifiés avec la même précision, des conflits vont forcément éclater du fait de l'importance de l'enjeu, et produiront des gagnants et des perdants, chaque aspect du problème est en lui-même un autre problème, aucune « solution » démontrable n'existe, seules sont possibles des « améliorations » itératives.

Souvenons-nous que le monde de l'agronomie a connu, ces dernières années, des appels en faveur d'un surcroît de recherches sur le réchauffement climatique, le développement rural, l'amélioration de l'environnement, la croissance économique, la durabilité et même la santé publique (Busch, 2009). Alors qu'il était auparavant possible de mettre ces questions entre parenthèses (il suffit de se remémorer la réaction initialement hostile de la communauté des entomologues dans les années 1960 à l'idée que la lutte chimique contre les ravageurs pouvait poser problème), c'est aujourd'hui un luxe que nous ne pouvons plus nous permettre (et que nous n'avons peut-être jamais pu). 


\section{Encadré 1.3. Exemple de réorientation de la recherche agronomique au bénéfice de petits producteurs au Brésil}

\section{Eliane de Carvalho Noya, Bernard Roux et Geraldo Majella Bezerra Lopes}

Dans le nord-est brésilien, l'Institution publique de recherche agronomique de l'État de Pernambuco (IPA) a récemment mis en œuvre une méthode de développement agricole basée sur le concept d'unité de recherche et d'apprentissage collectifs (UPAC), encouragée par des dispositions de politique publique du gouvernement Lula. Elle consiste à rechercher, en collaboration avec les organisations des petits producteurs agricoles familiaux, l'intégration des connaissances et des expériences des acteurs concernés localement par la production d'aliments : l'agriculteur, sa famille, l'agent de vulgarisation, le chercheur, l'enseignant, le leader local. Pour apporter une alternative aux anciennes méthodes de « diffusion des paquets technologiques » et parvenir à cette intégration, des activités collectives de recherche multidisciplinaire et d'apprentissage sont réalisées au moyens de visites, d'observations participatives, d'entrevues avec des personnes clés, des réunions de réflexion pour le diagnostic et le suivi de la situation réelle des communautés agricoles. L'objectif étant que ces connaissances puissent être utiles pour élaborer ou améliorer de nouveaux produits, procédés ou services. Des innovations technologiques et sociales endogènes et exogènes sont identifiées, systématisées, améliorées et validées, concernant des thèmes variés tels l'aquaculture, la viticulture, l'horticulture, la polyculture, l'économie solidaire, l'associativisme, etc. Cela permet d'identifier des éléments géographiques, socio-économiques, culturels, etc. qui passent habituellement non inaperçus des chercheurs très spécialisés.

L'UPAC s'inspire d'une approche systémique comportant les enjeux et résultats suivants :

- la participation de tous à la construction des connaissances concernant la production de denrées, visant la sécurité alimentaire de la communauté ;

- la justice sociale, l'égalité des chances, quel que soit le sexe ou groupe social, en relation avec l'emploi et le revenu ;

- la durabilité du processus de développement, au bénéfice des générations présentes et futures ;

- la valorisation et la conservation des ressources locales ;

- le contrôle des personnes sur leur destin en tant que citoyens.

Des résultats encourageants ont été obtenus. En particulier, le processus d'apprentissage mutuel entre les vulgarisateurs, les chercheurs et les agriculteurs fonctionne effectivement. Ces derniers trouvent des éléments pour la résolution de leurs problèmes par le dialogue avec ces acteurs ainsi qu'avec les collectivités locales, et les agents financiers. Les chercheurs et les vulgarisateurs modifient radicalement leur comportement. Ainsi, tous ceux qui participent à l'UPAC contribuent au développement des recherches, des connaissances, de l'information et des technologies dans un processus de co-construction du développement agricole et local, grâce à l'amélioration du capital humain et social. C'est une question de changement de méthode, de contenu et d'approche de la recherche agronomique (de Carvalho Noya et al., 2010).

Repenser et, surtout, remodeler la recherche agronomique se révèleront sans doute déstabilisant pour certains membres de la communauté scientifique. Je pense ici à 
ces chercheurs adeptes de la loi de Kaplan (1964) : donnez un marteau à un enfant et il trouvera que tout a besoin d'être martelé. Dans l'état actuel des choses, la plupart des chercheurs en sciences agricoles passent cinq années ou plus à tout apprendre sur un champ d'investigation particulier, très étroit, de l'agronomie. Beaucoup sont convaincus, à tort, que presque tous les problèmes peuvent être résolus grâce à leur spécialité, qu'il s'agisse de sélection des plantes, de biologie moléculaire ou d'économie agricole. Il nous faut réorganiser l'enseignement supérieur agronomique de manière à ce que les étudiants puissent approfondir une discipline particulière sans perdre le contact avec les autres.

Nous devons commencer à aborder nos recherches dans la perspective des chaînes de valeur et des « bassins alimentaires ${ }^{9}$ plutôt que du point de vue unique de la productivité. Considérons par exemple le cas du phosphore. La planète dispose de très peu de phosphore, alors qu'il s'agit là d'un macroélément essentiel pour la nutrition des végétaux. Pourtant, de grandes quantités sont gaspillées année après année (voir par exemple Quinton et al., 2010). Un programme de recherche véritablement interdisciplinaire pourrait par exemple soulever les questions suivantes :

- comment rendre plus efficace l'utilisation du phosphore par les plantes?

- quelles politiques instaurer qui seraient susceptibles d'encourager une utilisation plus économe et un recyclage du phosphore?

- quels changements introduire dans la fabrication et l'utilisation des engrais qui permettraient d'économiser le phosphore?

- quels sont les coûts et les impacts de la pollution au phosphore pour les acteurs « avals »?

- serait-il possible de réduire les quantités de phosphore consommées dans le cadre de ses autres utilisations afin d'accroître celles disponibles pour l'agriculture ?

Et pourtant, même approchée de cette manière, la question reste cantonnée en amont, car le problème fondamental de la durabilité est encore laissé de côté. Un programme de recherche avec cette ambition devrait s'attaquer à toute la chaîne de valeur alimentaire, depuis la production des intrants jusqu'à l'assiette du consommateur. De plus, comme la quantité de nourriture produite sur la planète est déjà suffisante pour nourrir tous ses habitants, la logique voudrait que l'accent soit initialement placé sur les problèmes rencontrés après récolte plutôt que sur l'accroissement de la productivité. Le droit à l'alimentation consacré par la législation internationale devra être pris en considération avec tout le sérieux requis (United Nations, 1948, § 25 (1)). Un tel programme de recherche aborderait ainsi les interrogations suivantes :

- de quelle manière pourrait-on, en jouant sur les transports, la transformation, la commercialisation et les autres pratiques, et en mettant en place des politiques idoines, réduire les pertes considérables qui interviennent après récolte à l'échelle planétaire?

- comment résoudre le problème des besoins alimentaires spécifiques des petits paysans, des ouvriers agricoles et des citadins pauvres ? Plus précisément, quelles modifications des pratiques de la chaîne alimentaire, quels changements tech-

9. Foodsheds ou «bassins alimentaires » : «bassins versants » de l'approvisionnement en denrées alimentaires [Note du traducteur]. 
niques et quels ajustements de politiques pourraient permettre d'assurer un approvisionnement alimentaire sûr, durable, suffisant et nutritivement approprié ? - quels sont les besoins d'ordre physique, juridique, politique et infrastructurel auxquels il faudrait répondre pour permettre un approvisionnement alimentaire durable pour tous?

S'attaquer à ces problèmes pernicieux nécessitera du courage politique et une volonté mobilisatrice, ainsi que des efforts concertés non négligeables transgressant les frontières réelles, bien qu'artificielles, des différentes disciplines. La communauté scientifique aura à mettre au point de nouvelles mesures et de nouvelles normes pour relever ce défi aux multiples facettes. La quête, de plus en plus don-quichottesque, d'une solution unique et universelle aux problèmes de l'alimentation et de l'agriculture devra être abandonnée. La recherche publique devra reprendre le contrôle de l'ordre du jour scientifique et réorienter les recherches de manière à renforcer la sécurité alimentaire. Il ne s'agit pas là de tâches faciles, et des échecs sont sans doute à prévoir le long du chemin. Mais, ne serait-ce que pour ces raisons, nous ne pouvons attendre plus longtemps. Pour paraphraser le poète américain Robert Frost, nous avons « des miles à parcourir avant de dormir ».

\section{" Références bibliographiques}

Akerlof G.A., 1970. The market for 'lemons': Quality uncertainty and the market mechanism. Quarterly Journal of Economics, 84, 488-500.

Anderson E., 1952 (actualisé en 1967). Plants, man and life, University of California Press, Berkeley.

Barre H.W., Call L.E., Kendall J.C., Mooers C.A., Allen E.W., Jardine J.T., 1928. Report of the committee on experiment station organization and policy: Continuity in research, In : Proceedings of the 42nd annual convention of land-grant colleges and universities, Washington, DC, 203-205.

Bartélemy G., 1979. Les jardiniers du Roy : Petite histoire du Jardin des plantes de Paris, Le Pélican, Paris.

Batie S.S., 2008. Wicked problems and applied economics. American Journal of Agricultural Economics, 90, 1176-1191.

Bingen J., Busch L., 2005. Agricultural standards: The shape of the global food and fiber system, Springer, Dordrecht.

Bourdieu P., 1998. L'essence du néoliberalisme. Le Monde Diplomatique, mars, http://mondediplo. com/1998/12/08bourdieu.

Brockway L.H., 1979. Science and colonial expansion: The role of the British Royal Botanic Gardens, Academic Press, New York.

Busch L., 2007. Performing the economy, performing science: from neoclassical to supply chain models in the agrifood sector. Economy and Society, 36, 439-468.

Busch L., 2009. What kind of agriculture? What might science deliver? Natures, Sciences, Sociétés, 17, 241-247.

Busch L., 2011. Standards: recipes for realit, MIT Press, Cambridge, MA.

Busch L., Sachs C., 1981. The agricultural sciences and the modern world system, In : Science and Agricultural Development (Busch L., ed.), Allanheld, Osmun, Totawa, NJ, 131-156.

Busch L., William B.L., 1983. Science, agriculture, and the politics of research. Westview Press, Boulder, CO.

Butterfield K.L., 1917 (actualisé en 1918). The Morrill Act Institutions and the New Epoch, In : Proceedings 31st convention association of american agricultural colleges and experiment stations, Washington, DC, 43-59. 
Calestous J., 1989. The gene hunters: biotechnology and the scramble for seeds, Princeton University Press, Princeton.

Cassidy J., 2009. How markets fail. The logic of economic calamities, Farrar, Strauss \& Giroux, New York.

Chebrolu S.P., Sen D., 2010. The new commons in agriculture: lessons from the margins and sri in India, In : Innovation and sustainable development in agriculture and food (ISDA), Montpellier, France - http://hal.archives-ouvertes.fr/hal-00521398/fr/

De Carvalho Noya E., Roux B., Majella Bezerra Lopes G., 2010. Changer est nécessaire, changer est- il possible ? Interaction entre acteurs et construction de nouvelles pratiques en science et technologie pour 1'agriculture familiale dans l'État de Pernambouc, Brésil, In : Innovation and sustainable development in agriculture and food (ISDA), Montpellier, France - http://hal.archivesouvertes.fr/hal-00521362/fr/

Diamond J., 2005. Collapse: How societies choose to fail or succeed, Viking, New York.

Dries L., Reardon T., Swinnen J.F.M., 2004. The rapid rise of supermarkets in central and eastern Europe: Implications for the agrifood sector and rural development. Development Policy Review, 22, 525-556.

Echeverria R.G., 1998. Agricultural research policy issues in latin America: An overview. World Development, 26, 1103-1111.

Evans G.A., 2000. Designer science and the "omic" revolution. Nature Biotechnology, 18, 127.

Fejer S.O., 1966. The problem of plant breeder's rights. Agricultural Science Review, 3ème trimester, 1-7.

Frey K., 1996. National Plant Breeding Study, I: Human and financial resources devoted to plant breeding research and development in the United States in 1994, Iowa Agriculture and Home Economics Experiment Station, Ames, IA.

Fuglie K., Ballenger N., Day K., Klotz C., Ollinger M., Reilly J., Vasavada U., Jet Y., 1996. Agricultural research and development: Public and private investments under alternative markets and institutions, USDA, Washington, DC.

Fulponi L., 2006. Private voluntary standards in the food system: The perspective of major food retailers. Food Policy, 31, 1-13.

Grammer A.R., 1947. A history of the experiment station of the hawaiian sugar planters' association: 1895-1945. The Hawaiian Planters' Record, 51, 177-228.

Hanafi S., 2011. University systems in the Arab East: Publish globally and perish locally vs. publish locally and perish globally. Current Sociology, 59, 291-309.

Harlan H.V., Martini M.L., 1936. Problems and results of barley breeding, United States Department of Agriculture, Washington, DC.

Haudricourt A.G., Hédrin L., 1987. L'Homme et les plantes cultivées, Editions A.-M. Métailié, Paris.

Huffman W., Evenson R., 2006. Do formula or competitive grant funds have greater impacts on state agricultural productivity? Am. J. Agric. Economics, 88, 783-798.

Kaplan A., 1964. The conduct of inquiry, Chandler, Scranton.

King F.H., 1911. Farmers of forty centuries, Rodale Press, Inc., Emmaus, PA.

Lemmens P., 2010. Deproletarianizing agriculture - recovering agriculture from agribusiness and the need for a commons-based, open source agriculture, In:Innovation and sustainable development in agriculture and food (ISDA), Montpellier, France - http://hal.archives-ouvertes.fr/hal00539829/fr/

McNeill W.H., 1963. The rise of the west, University of Chicago Press, Chicago.

Mutersbaugh T., 2005. Fighting standards with standards: harmonization, rents, and social accountability in certified agrofood networks. Environment and Planning A, 37, 2033-2051.

National Institute of Standards and Technology, 2007. Standard Reference Materials ${ }^{\circledR}$ Catalog. US Government Printing Office, Washington, DC.

Pardey P.G., Beintema N., Dehmer S., Wood S., 2006. Agricultural research: A growing global divide? International Food Policy Research Institute, Washington, DC. 
Pearl R., 1915. The publication of the results of investigations made in experiment stations in technical scientific journals, including the Journal of Agricultural Research, In : Proceedings of the 29th convention of the association of american agricultural colleges, Berkeley.

Perkins J.H., 1997. Geopolitics and the green revolution, Oxford University Press, New York.

Prest J., 1981. The garden of Eden: The botanic garden and the recreation of paradise, Yale University Press, New Haven.

Quinton J.N., Govers G., Van Oost K., Bardgett R.D., 2010. The impact of agricultural soil erosion on biogeochemical cycling. Nature Geoscience, 18, 311-314.

Reardon T.C., Timmer P., Barrett C.B., Berdegue J., 2003. The rise of supermarkets in Africa, Asia, and Latin America. Am. J. Agric. Economics, 85, 1140-1146.

Reboul C., 1977. Déterminants sociaux de la fertilité des sols. Actes de la Recherche en Sciences Sociales, 17/18, 85-112.

Rittel Hörst W.J., 1972. On the planning crisis: Systems analysis of the 'first and second generations'. Bedriftsøkonomen, 8, 390-396.

Rittel Hörst W.J., Webber M.M., 1973. Dilemmas in a general theory of planning Policy Sciences, 4, 155-169.

Rudy A.P., Coppin D., Konefal J., Shaw B.T., Eyck T.T., Harris C., Busch L., 2007. Universities in the age of corporate science: The UC Berkeley-Novartis Controversy, Temple University Press, Philadelphie.

Ruivenkamp G., Jongerden I., 2010. Open source and commons in development, In : Innovation and sustainable development in agriculture and food (ISDA), Montpellier, France - http://hal.archives-ouvertes.fr/hal-00521937/fr/

Tansey G., Rajotte T., 2008. The future control of food, Earthscan, Londres.

Thomas W.I., 1928. The child in America: Behavior problems and programs, Alfred A. Knopf, New York.

United Nations, 1948. Universal Declaration of Human Rights, Nations Unies, New York.

Weatherspoon D.D., Reardon T., 2003. The rise of supermarkets in Africa: Implications for agrifood systems and the rural poor. Development Policy Review, 21, 1-17.

Weis T., 2007. The global food economy: The battle for the future of food and farming, Zed Books, Londres.

Wolf S., Zilberman D., 2001. Knowledge generation and technical change: Institutional innovation in agriculture, Kluwer Academic Publishers, Boston. 



\title{
Chapitre 2 \\ Systèmes d'innovation : \\ du concept à la pratique émergente
}

\author{
Bernard TRIOMPHE et Riikka RAJALAHTI
}

$\mathrm{Au}$ cœur des changements environnementaux et socio-économiques profonds et complexes qui se produisent tant sur le plan local qu'à l'échelle mondiale, les agriculteurs, les entreprises du secteur agroalimentaire et les sociétés doivent constamment innover pour pouvoir faire face aux évolutions actuelles, rester compétitifs et prospérer. Le développement agricole durable, en particulier, demande toute une palette d'innovations techniques, sociales et institutionnelles.

Comment mieux innover a été une question amplement débattue. Pendant des décennies, beaucoup d'acteurs du secteur agricole ont plaidé et considérablement investi pour le développement et le renforcement de la recherche publique, des services de conseil agricole, de l'enseignement, et des liens les connectant les uns aux autres, dans l'idée de créer et de diffuser les connaissances et la panoplie de technologies perçues comme indispensables au développement agricole. Le modèle d'innovation sous-jacent était alors essentiellement linéaire : dans un tel modèle, les chercheurs produisent et utilisent des connaissances pour générer de nouvelles technologies qui sont ensuite diffusées par les conseillers agricoles auprès des agriculteurs potentiellement intéressés. Pour encourager les agriculteurs à adopter ces innovations, les conseillers agricoles leur fournissent une assistance sous forme de conseils, de crédits, de formations, etc. (Rodgers, 1983). Ces investissements et ces approches ont produit des résultats significatifs, de nombreux agriculteurs et consommateurs en tirant bénéfice, ainsi que l'a mis en évidence l'effet - très controversé quoique incontestable - de la révolution verte (Evenson et Gollin, 2003). Cependant, leur impact ne s'est pas toujours révélé suffisant, en particulier pour les acteurs et les milieux les plus démunis et marginalisés. Ils n'ont pas non plus su faire face aux myriades de demandes, de défis et d'opportunités qui émanent d'un ensemble d'acteurs de plus en plus diversifié au sein d'un environnement en constante mutation (Rajalahti et al., 2005, 2008 ; Banque mondiale, 2012).

Le succès limité des approches linéaires de l'innovation, ainsi que l'expérience et les leçons accumulées sur la manière dont l'innovation advient dans la pratique, ont suscité l'émergence et, surtout, l'adoption et la mise en œuvre progressive d'approches s'inspirant du concept des systèmes d'innovation (Freeman, 1988 ; Lundvall, 1992 ; OCDE, 1997). Initialement utilisé dans le cadre du développement des technologies 
dans les économies développées, le concept des systèmes d'innovation a ensuite été adapté et appliqué au secteur agricole, tant dans les pays développés que dans les pays en voie de développement (Banque mondiale, 2006). L'approche fondée sur les systèmes d'innovation agricoles ou approche SIA (Agricultural Innovation Systems Perspective) qui découle de cette évolution s'est révélée très utile, par exemple pour évaluer les forces et les faiblesses de processus, systèmes et environnements d'innovation particuliers et, de plus en plus, pour planifier et mettre en œuvre des actions susceptibles de contribuer à des systèmes d'innovation plus fonctionnels et dynamiques et à un plus grand impact des investissements dans l'innovation.

Ce chapitre présente l'approche SIA en mettant en exergue sa contribution à l'analyse et à la conception de l'innovation agricole. Il débute par un bref récapitulatif de l'apparition du concept de système d'innovation et de l'approche qui en découle, y compris les liens que ce concept entretient avec d'autres tels que la théorie de l'acteur-réseau. Sont ensuite passés en revue une série d'approches et d'instruments qui contribuent tous à rendre l'approche SIA opérationnelle et à lui donner un contenu concret. Enfin sont évoqués quelques-uns des problèmes auxquels se heurte actuellement l'application de l'approche SIA, tandis que la conclusion suggère quelques axes à privilégier pour les efforts à venir en matière de SIA.

\section{" Le concept de système d'innovation et son origine}

\section{Aperçu historique}

Pour le dire simplement, l'innovation est le processus par lequel des individus ou des organismes maîtrisent et mettent en ouvre la conception et la production de biens et de services qui leur sont nouveaux. Elle peut également être définie comme une invention ayant trouvé un marché (Schumpeter, 1942), une distinction intéressante en ce qu'elle différencie le contenu d'une innovation, essentiellement constitué de connaissances (le surgissement d'une nouvelle idée), de son application.

À se pencher sur l'historique et la trajectoire des innovations, on retombe très régulièrement sur l'idée ou la leçon selon laquelle la « réussite » d'une innovation peut bien souvent être attribuée à l'existence de réseaux efficaces grâce auxquels les chercheurs et les entrepreneurs des secteurs public et privé ont collaboré, appris les uns des autres, partagé des ressources et réagi rapidement aux mutations des conditions économiques et techniques (Banque mondiale, 2006). En particulier, le succès des économies telles que le Japon dans les années 1970 et 1980 a suscité beaucoup d'attention, les autres pays développés aspirant à s'engager dans la même voie. En étudiant ces économies, Freeman (1988) a été le premier à employer l'expression « système national d'innovation » pour désigner les institutions d'État associées à la définition et à l'application des politiques de recherche et d'innovation. Dans d'autres travaux consacrés aux systèmes nationaux d'innovation au début des années 1990, Nelson (1993) et Lundvall (1992) ont étendu la définition de Freeman jusqu'à y inclure des acteurs du secteur industriel et des éléments du contexte national accueillant des activités de recherche et d'innovation. Tout au long des années 1990 à 2000, l'Organisation de coopération et de développement économiques (OCDE) a 
utilisé de plus en plus fréquemment l'expression « conditions-cadres » pour décrire la portée des systèmes d'innovation, à savoir les facteurs contextuels tels que les régimes fiscaux, les réglementations, la législation, la culture et les comportements, élargissant ainsi plus encore la définition du système d'innovation (OCDE, 1997).

Des auteurs tels que Latour (1987), Akrich et al. (1988) et Callon et al. (2001) ont à leur actif plusieurs contributions décisives, quoique pas toujours reconnues, au concept de système d'innovation tel qu'on le connaît actuellement, par le biais de leurs travaux fondamentaux sur la théorie de l'acteur-réseau, ou ANT (Actor-Network Theory). L'ANT permet de mieux comprendre l'éclosion, le fonctionnement et la structure de réseaux d'innovation liant entre eux un ensemble diversifié d'acteurs et d'objets avec lesquels ils interagissent au cours du processus de mise au point de l'innovation. Le processus d'innovation est ainsi vu comme le résultat d'ajustements réciproques, co-évolutifs et organiques entre l'objet de l'innovation et la société qui l'entoure, qui suivent une trajectoire non linéaire et imprévisible. L'ANT permet en outre d'analyser les diverses incitations et motivations qui poussent à innover, et, inversement, les obstacles et les différentes formes de résistance auxquels l'innovation est susceptible d'être confrontée au cours de son histoire. Enfin, l'ANT met en évidence le rôle central des médiateurs - des acteurs qui présentent les compétences et la puissance nécessaires pour dégager une position commune entre les parties prenantes sur les significations et les interprétations dans le cadre d'un processus de traduction qui fait partie intégrante du processus d'innovation.

Le concept de système d'innovation a commencé par être appliqué à l'échelle nationale avant de l'être à d'autres échelles internationale, régionale, locale ou sectorielle. Les acteurs associés à l'innovation, ainsi que le contexte même du système, varient d'une échelle à l'autre. Le temps constitue une autre dimension clé de l'analyse, étant donné que les systèmes d'innovation évoluent de manière dynamique sur des périodes souvent relativement longues, ainsi que l'ont amplement démontré les travaux consacrés à l'ANT (par exemple Alter, 2000). Certains chercheurs travaillant sur les systèmes d'innovation ont proposé de traiter cette dimension temporelle en différenciant les phases de démarrage, d'émergence, de paysage et de régime (Knickles et al., 2008), ou les phases de démarrage, d'émergence et de stagnation (Banque mondiale, 2006). En sus de l'échelle et de la dimension temporelle, il est utile de préciser si l'innovation est le résultat d'un processus planifié ou orchestré, ou au contraire d'un processus plus spontané et non orchestré (Banque mondiale, 2006) - une situation récemment désignée par l'expression "écosystème d'innovation » par certains auteurs (Fukuda et Watanabe, 2008).

Dans ce contexte historique, un système d'innovation peut être défini comme « un réseau d'organisations, d'entreprises et d'individus focalisés sur l'exploitation économique de nouveaux produits, procédés et formes d'organisation, ainsi que les institutions et les politiques qui influencent leur comportement et leur performance » (Banque mondiale, 2006).

Si certains auteurs ont proposé des définitions légèrement différentes du système d'innovation, selon les aspects sur lesquels ils se penchaient plus particulièrement (par exemple Freeman, 1987 ; Lundvall, 1992 ; Metcalfe, 1995), toutes les définitions existantes ont cependant en commun de proposer un cadre systémique pour appréhender le réseau relativement complexe des acteurs et des processus à l'œuvre 
dans le développement technologique (OCDE, 1997). Deux aspects clés sont ainsi mis en exergue : la multiplicité des acteurs mis en jeu et leurs interactions, et l'environnement dans lequel ces acteurs opèrent.

\section{Les systèmes d'innovation et le secteur agricole}

Le secteur agricole n'est qu'un exemple parmi d'autres des champs d'application du concept de système d'innovation pour renouveler notre compréhension de la manière dont l'innovation se développe et peut être cultivée. Dans un SIA (figure 2.1), l'innovation surgit généralement à la faveur d'une interaction dynamique et ouverte entre les nombreux acteurs qui interviennent dans la production, la transformation, le conditionnement, la distribution et la consommation ou l'utilisation des produits de l'agriculture, ce qui permet de tirer parti des connaissances disponibles les plus appropriées. La participation de la recherche, des services de conseil agricole et de l'enseignement est habituellement nécessaire, mais pas suffisante pour apporter aux agriculteurs et aux entrepreneurs les savoirs, les technologies et les services dont ils ont besoin. Outre de robustes capacités en matière de recherche-développement, la capacité à innover va souvent de pair avec l'action collective, la coordination, l'échange de connaissances entre acteurs, les mesures incitatives et les ressources disponibles pour forger des partenariats et développer des entreprises, et l'existence de conditions qui autorisent les agriculteurs et les entrepreneurs à faire usage des innovations (Hall et al., 2003 ; Banque mondiale, 2012).

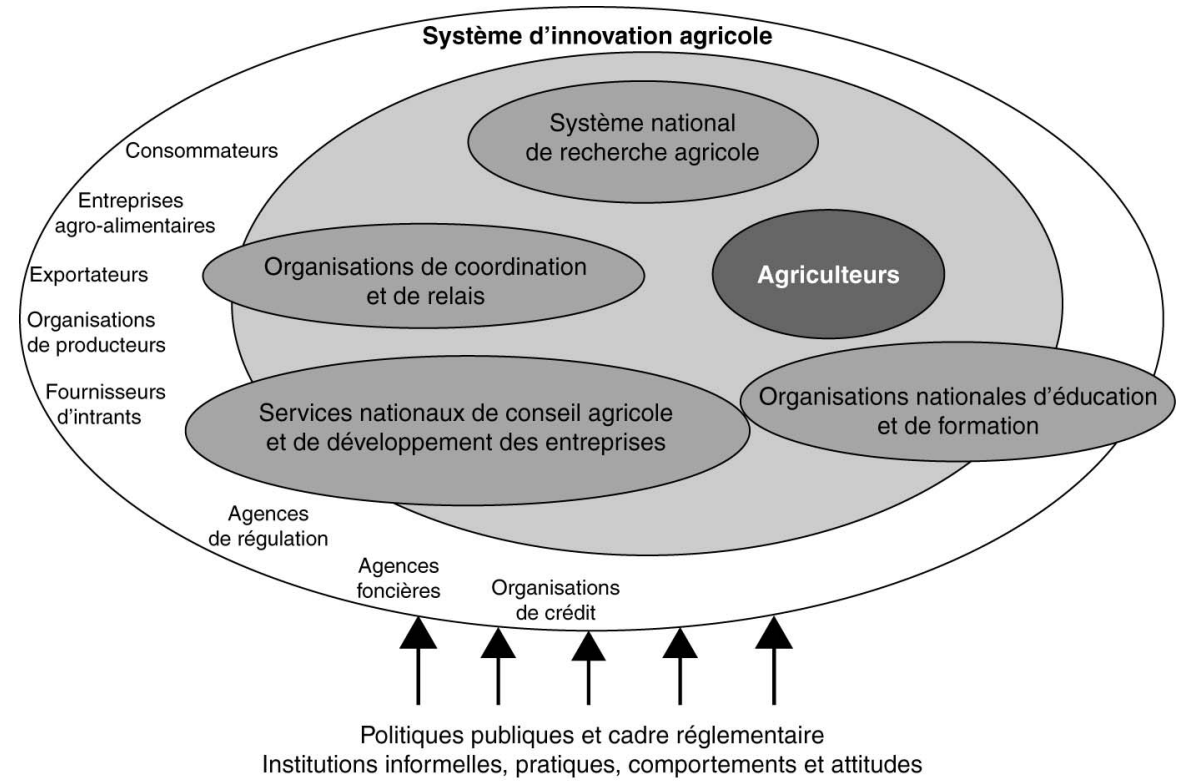

Figure 2.1. Représentation schématisée d'un système d'innovation agricole (source : Banque mondiale, 2012). 
Par rapport aux approches et aux paradigmes antérieurs, le concept des SIA permet de disposer d'un cadre élargi permettant de multiplier les manières d'appréhender l'innovation agricole (Hall et al., 2003 ; Banque mondiale, 2006). Tout d'abord, contrairement au paradigme linéaire, il ne se concentre ni étroitement ni prioritairement sur l'aspect offre en innovation (le fameux technology pull), ni non plus sur l'aspect demande (le technology push), mais prend plutôt en considération les initiatives provenant de l'ensemble des acteurs et des parties prenantes, qu'il s'agisse d'individus ou d'institutions, de concepteurs ou d'utilisateurs et qu'ils appartiennent au secteur public ou privé. Le cadre SIA tient également compte des connaissances, des compétences et des attitudes de chacun de ces acteurs, qu'elles contribuent positivement à l'innovation ou, parfois, qu'elles s'y opposent. Il accorde aussi une attention soutenue aux interactions qui existent entre les divers acteurs, y compris aux aspects de coordination, de gouvernance et d'intermédiation ( « courtage »). Le marché est dûment reconnu comme une force très puissante pour susciter et entretenir l'innovation agricole, étant donné que les initiatives innovantes naissent souvent à la faveur de nouvelles opportunités - ou de nouveaux signaux - du marché. Dernier point, mais non des moindres, le concept des SIA met en évidence les relations cruciales qui existent entre les processus d'innovation et l'environnement ou le contexte socio-économique dans lequel ils s'inscrivent, ce qui comprend les aspects politiques, qui sont fondamentaux.

\section{Les systèmes d'innovation agricole et la Banque mondiale}

En ce qui la concerne, la Banque mondiale s'est progressivement convertie au concept des SIA. Tout au long des années 1970 et jusqu'à un passé relativement récent, elle a beaucoup investi dans la construction et le renforcement de systèmes nationaux de recherche agricole (Byerlee et Alex, 1994) ainsi que dans le renforcement des services de conseil agricole par la mise en application de l'approche Training and Visit ( formation et visite ») du transfert de technologie (Benor et Baxter, 1984 ; Hussein et al., 1994). La recherche et la vulgarisation étaient en effet, à l'époque, considérées comme les deux grands piliers du développement agricole, en accord avec le paradigme alors en vigueur de mise au point et de transfert de technologies. La prise de conscience graduelle, qui transparaît dans l'approche par les systèmes d'information et de connaissances agricoles (Agricultural Knowledge and Information Systems ou AKIS) (Röling, 1990), de l'existence d'un paysage plus large de connaissances et d'innovation, puis le travail fondateur qui a conduit à la publication de l'ouvrage Enhancing Agricultural Innovation (Banque mondiale, 2006) ont contribué au développement et à l'adoption du concept de système d'innovation. Le concept de SIA a par la suite vu sa position renforcée par un projet collectif de grande ampleur qui a récemment abouti avec la publication du manuel Agricultural Innovation Systems, An Investment Sourcebook (Banque mondiale, 2012). Cet ouvrage, en s'appuyant sur l'expérience acquise à l'échelle mondiale, expose le contexte théorique et propose des conseils et des exemples fondés sur des données bien établies à destination des investisseurs et des porteurs de projets dans le domaine des SIA dans des contextes agricoles et d'innovation variés. Il aborde des aspects fondamentaux tels que la coordination des politiques et l'action collective, l'enseignement et la formation, les services de conseil et de vulgarisation, les 
systèmes de recherche, l'engagement du secteur privé, les environnements porteurs d'innovation, ainsi que le suivi et l'évaluation des investissements dans les SIA.

\section{W Faire passer l'approche des systèmes d'innovation au stade opérationnel}

Une des raisons pour lesquelles l'approche SIA se répand rapidement (voir UE SCAR, 2012, pour une récapitulation récente de ce phénomène au niveau de l'Europe) a peut-être à voir avec le fait qu'elle a permis à plusieurs écoles de pensée et à plusieurs disciplines de converger au sein d'un cadre de travail commun qui permet de diagnostiquer simultanément les processus et les systèmes d'innovation en cours et d'agir sur eux de manière pertinente. Nous affirmons ici que cette opérationnalisation repose sur le recours à une série d'approches et d'instruments interconnectés qui, chacun à leur manière, sont susceptibles de contribuer à un fonctionnement satisfaisant des systèmes d'innovation présents et futurs. Ces approches et ces instruments offrent des moyens concrets d'atteindre des objectifs aussi importants que le renforcement et la facilitation des interactions entre la recherche et les autres acteurs, l'intégration des innovations dans les marchés, le développement de partenariats public-privé, la coordination d'une multiplicité de parties prenantes, le financement de l'innovation, ou encore le transfert de technologie. Ils sont souvent employés de manière combinée dans le cadre d'une même initiative SIA. Qui plus est, il serait illusoire d'établir une hiérarchie claire entre approches et instruments : si les approches ont habituellement une connotation plus générique, un « instrument» (par exemple, un programme d'allocations concurrentielles) peut dans bien des cas répondre en réalité à un objectif de niveau supérieur à celui d'une « approche » (telle que l'approche par les chaînes de valeur, ou filières). Plusieurs de ces approches et instruments seront brièvement présentés et illustrés dans cette section.

\section{Renforcer les interactions entre la recherche et les autres acteurs}

Bien que dans l'optique des SIA, la recherche ne soit qu'un acteur parmi tous ceux qui interviennent dans le processus d'innovation, elle y tient un rôle clé. Depuis plusieurs décennies déjà, des efforts ont été consentis pour tenter d'améliorer la performance de la recherche ainsi que sa pertinence et sa contribution au développement de l'innovation. Ces efforts continuent aujourd'hui, bien qu'ils aient dû être adaptés à un environnement très différent de ce qu'il était autrefois.

En grande partie fondés au cours des années 1980 et 1990, les divers courants de la recherche sur les systèmes agraires et de production (Farming System Research, ou FSR) (Norman et Collinson, 1985 ; Jouve et Mercoiret, 1987) et de la recherche participative (Participative Research, ou PR) (Chambers et al., 1989 ; Ashby et al., 1995) ont contribué à concentrer l'attention sur les agriculteurs en tant qu'acteurs de premier plan de l'innovation agricole. Dans les approches de ce type, les agriculteurs sont considérés dans toute leur diversité pour ce qui est de leurs caractéristiques essentielles et de leurs besoins, et aussi pour ce qui est de l'environnement dans 
lequel ils évoluent. La FSR et la PR ont beaucoup aidé à populariser l'application de cette approche systémique en agriculture à différentes échelles (comme le reflètent les notions de système de culture, système de production et système agraire), le concept de système d'innovation en étant à la fois un prolongement direct et une extension. Récemment, ces approches ont été élargies et combinées aux principes de la recherche-action pour donner naissance à une panoplie de méthodes rassemblées sous l'étiquette toute récente de co-conception (Béguin, 2003 ; Triomphe, 2012a, par exemple). La co-conception s'inscrit particulièrement bien dans le cadre des SIA dans la mesure où elle reconnaît et met en avant à la fois la grande diversité des acteurs (ou des parties prenantes) avec lesquels la recherche collabore et la nature intrinsèquement itérative et non linéaire du développement de l'innovation.

Les acteurs du développement ont pour leur part proposé des approches semblables, telles que le développement technologique participatif (Participatory Technology Development) et, plus récemment, le développement participatif de l'innovation (Participatory Innovation Development) (Hagmman et al., 1996 ; Veldhuizen et al., 1997 ; Biggs et Smith, 1998, par exemple). Proches cousins de la PR, de la FSR et de la co-conception, elles sont toutefois beaucoup plus focalisées sur la manière d'organiser et de stimuler concrètement les processus de développement technologique ou de développement de l'innovation, donnant ainsi la première place non pas à la recherche mais aux agriculteurs et aux autres parties prenantes du monde rural, ainsi qu'aux organisations de développement, y compris les ONG.

Pour ce qui est de la recherche-action, il s'agit là d'une approche relativement ancienne, dont les origines sont extérieures à l'agriculture (Lewin, 1946 ; Liu, 1997 par exemple). Elle a toutefois été adaptée depuis pour une application dans le contexte agricole (Albaladejo et Casabianca, 1997 ; Faure et al., 2010). La rechercheaction apporte un cadre intéressant et des lignes directrices génériques pour pouvoir articuler de manière fonctionnelle la production de connaissances (par la recherche, mais également par des utilisateurs œuvrant à titre de chercheurs profanes) et la résolution concrète de problèmes ou de désirs de changement rencontrés ou exprimés par les utilisateurs. Elle met également en exergue le besoin d'élaborer une approche très souple, itérative et réflexive du développement de l'innovation, qui s'appuie sur la planification et l'apprentissage conjoints entre les diverses parties concernées. Un autre problème fondamental que la recherche-action peut aider à résoudre est celui de la coordination et de la gouvernance concrètes d'un ensemble diversifié de parties prenantes. Elle montre en outre l'importance du rôle d'un corpus commun de valeurs et de principes éthiques pour mettre en place une action collective efficace. Enfin, mais ce n'est pas le moins important, la mise au point de nouvelles institutions et de nouvelles politiques - et pas seulement de nouvelles technologies - est une préoccupation majeure de la recherche-action et constitue un des résultats phares de son application.

Changer la manière dont la recherche est conduite actuellement est certes fondamental pour pouvoir mettre en pratique une approche SIA, mais on rencontre encore fréquemment, notamment dans les pays en voie de développement, des situations où la recherche et les autres acteurs publics ou même privés qui seraient capables et désireux d'investir dans le développement de l'innovation sont pratiquement absents du paysage. Dans ces conditions, il peut s'avérer nécessaire, pour mettre en œuvre une approche SIA, que les quelques institutions de soutien actives sur 
place (y compris les organisations non gouvernementales, ONG) consacrent des efforts significatifs à la stimulation de l'innovation locale et, partout où la chose est possible, qu'elles s'engagent dans des actions de recherche conjointe sous la houlette des agriculteurs. L'encadré 2.1 présente la méthode utilisée à cette fin par le réseau PROLINNOVA dans plusieurs pays en voie de développement.

\section{Encadré 2.1. Stimuler l'innovation locale et la recherche conjointe orientée par les agriculteurs}

Dans l'approche SIA, des initiatives d'innovation sont susceptibles d'émaner de n'importe laquelle des parties prenantes. Pourtant, le rôle crucial des agriculteurs eux-mêmes en tant qu'innovateurs et co-producteurs de connaissances demeure trop souvent ignoré. PROLINNOVA (Promoting Local Innovation) est un réseau international d'apprentissage et de promotion qui croit dans les changements que les décideurs-clés que sont les agriculteurs, femmes et hommes, peuvent apporter dans les domaines de l'agriculture durable et de la gestion des ressources naturelles. PROLINNOVA s'efforce de développer des partenariats et des approches dans le cadre desquels le processus de développement d'innovations s'amorce autour de nouvelles idées mises au point par les agriculteurs.

À l'heure actuelle, le réseau PROLINNOVA s'étend sur 20 pays d'Asie, d'Afrique et d'Amérique latine. Les activités menées aux échelles nationale et régionale bénéficient du soutien de l'IIRR (Institut international de reconstruction rurale), de l'ETC (Educational Training Consultancy) et de l'IED-Afrique (Innovation environnement développement). Les membres de PROLINNOVA sont issus d'organismes publics de recherche et de vulgarisation, d'ONG, d'établissements d'enseignement, d'associations locales, d'associations d'agriculteurs et du secteur privé. Les membres des réseaux nationaux sont encouragés à repérer, documenter et faire connaître les innovations et innovateurs locaux, qui sont souvent non décelés et non exploités par l'extérieur. Ils mettent également en place des partenariats avec des agriculteurs locaux et leurs organisations afin de soutenir les processus d'innovation locale en cours. Ces activités comprennent la mise en œuvre de programmes d'expérimentation conjointe sous la houlette des agriculteurs pour perfectionner les innovations locales, la mise en commun des expériences et leur diffusion à grande échelle (par le biais de foires de l'innovation, des médias, etc.), et le renforcement des capacités de l'ensemble des acteurs concernés à mettre en ouvre des approches participatives appropriées. Un moyen critique pour permettre aux agriculteurs de prendre part aux activités de recherche et d'innovation agricoles est de leur ouvrir un accès à des fonds décentralisés pour l'innovation. Des partenaires de PROLINNOVA pilotent à cet effet la mise en place de mécanismes de financement décentralisés (les Local Innovation Support Funds, fonds de soutien à l'innovation locale) dans huit pays. Les premiers résultats mettent en évidence l'utilité et le potentiel de ces programmes pour dynamiser l'innovation locale et la recherche conjointe dirigée par les agriculteurs.

Les membres du réseau partagent régulièrement à l'échelle nationale et internationale les enseignements tirés de leurs expériences, sur lesquels ils s'appuient pour promouvoir et soutenir la capacité d'innovation des petits agriculteurs. Ils participent par ailleurs activement au dialogue politique à divers niveaux pour aiguillonner et renforcer l'innovation locale et pour contribuer à créer des politiques propices au développement participatif de l'innovation (Wongtschowski et al., 2010 ; Banque mondiale, 2012). 


\section{Améliorer l'intégration et l'accès aux marchés}

Peut-être sans surprise - l'agriculture s'intégrant fortement à la société de plus en plus urbanisée et mondialisée qui l'entoure - de nombreuses innovations récentes et en cours dans le secteur agricole sont développées dans l'espoir de favoriser ou d'exploiter une plus grande intégration aux marchés, ou simplement un meilleur accès aux marchés. Un objectif important est de relier de façon plus fonctionnelle la production à la demande des consommateurs et des utilisateurs finaux.

L'expérience du programme Papa Andina (encadré 2.2), fondé sur l'approche participative des filières (Participatory Market Chain Approach ou PMCA) (Devaux et al., 2009), fournit un exemple parlant pour illustrer comment les processus d'innovation axés sur les marchés peuvent être facilités et mis en œuvre.

\section{Encadré 2.2. Papa Andina et l'approche participative des chaînes de marchés}

Dans le cadre d'une approche SIA, l'accès au marché est souvent un ingrédient essentiel du succès. Comme l'a déclaré Victoriano Meza, agriculteur à Pomamanta, un village des Andes péruviennes, «vendre mes pommes de terre de variétés locales à l'industrie a changé notre vie ». Il est un agriculteur parmi plusieurs centaines d'autres qui tirent profit d'une nouvelle tendance du marché en faveur des pommes de terre locales. Pour Victoriano Meza, ceci se traduit par de meilleurs revenus, qui lui ont permis de construire une nouvelle maison pour sa famille et de l'équiper pour avoir un accès à Internet par satellite " pour que mes enfants puissent s'instruire rapidement et bénéficient d'un meilleur avenir ».

Cet essor soudain du marché résulte de l'application de l'approche participative des chaînes de marchés (Participatory Market Chains Approach ou PMCA) dans le cadre du programme Papa Andina, un programme de partenariat fondé en 1998 et financé par la Suisse dans le but de promouvoir l'innovation en faveur des pauvres dans les chaînes de marchés, avec des partenaires nationaux en Bolivie, en Equateur et au Pérou. Un des objectifs de l'initiative Papa Andina était d'améliorer les liens au sein de la filière de la pomme de terre et en particulier d'aider les petits agriculteurs à accéder aux marchés et aux opportunités que ces derniers peuvent leur apporter pour renforcer leurs moyens d'existence. Une première phase de PMCA a débuté en 2002 dans le but de mettre au point de nouveaux produits de la pomme de terre. Une seconde phase a concerné les variétés locales de pomme de terre, qui sont principalement cultivées sur de petites exploitations d'altitude dans des régions reculées des Andes. Dans les deux cas, des groupes de travail multi-acteurs facilités ont été constitués pour analyser les opportunités de marché et développer de nouveaux produits.

Parmi les principaux résultats de ces deux phases de PMCA, on peut citer la création d'une nouvelle marque de pommes de terre non transformées de qualité supérieure pour le marché de gros, d'un nouveau produit (et d'une nouvelle marque) de pommes de terre frites de variété locale, et la première marque de pommes de terre locales de qualité supérieure destinée à la commercialisation en grande surface au Pérou. Des innovations à caractère technique ont également été produites en matière de lutte contre les ravageurs et les maladies, et de sélection de la production après récolte. Une plate-forme nationale, la CAPAC-Pérou, a par ailleurs été mise en place pour promouvoir la commercialisation de la pomme de terre de qualité et 
pour favoriser l'innovation. La CAPAC, au sein de laquelle les acteurs locaux prennent progressivement plus de responsabilités au fur et à mesure que leurs capacités et leur confiance se développent, a aidé de petits agriculteurs à s'organiser pour fournir des pommes de terre répondant aux exigences de marché particulières de T'ikapapa. Rapidement, d'autres acteurs ont commencé à prendre conscience du potentiel encore sous-exploité des pommes de terre de variétés locales sur les marchés urbains et ont commencé à mettre au point de nouveaux produits à base de ces tubercules. Aujourd'hui, des pommes de terre et des frites de pomme de terre de qualité supérieure sont commercialisées sous différentes marques dans la plupart des grandes chaînes péruviennes de grandes surfaces. Suite à l'entrée d'une multinationale sur ce marché, Papa Andina a commencé à travailler sur la responsabilité sociale des entreprises, ce qui a permis d'équilibrer les intérêts des entreprises avec ceux des petits fournisseurs ruraux et ceux de l'environnement. Dernière chose, mais non des moindres, une journée annuelle nationale de la pomme de terre a été instaurée au Pérou.

Ces nombreuses innovations et évolutions ont contribué à une progression des revenus et de l'estime de soi des agriculteurs, une stabilisation des marchés, une meilleure organisation des agriculteurs, une meilleure coordination des acteurs et la popularisation des variétés locales de pommes de terre dans les habitudes culinaires des citadins.

Il reste cependant des défis à relever. La coordination et la facilitation, par exemple, réclament des animateurs et des « courtiers » à temps plein ainsi que des investissements importants dans le domaine du renforcement des capacités. La consolidation de la viabilité financière en dépassant le financement des bailleurs de fonds constitue un autre problème, de même que les méthodes classiques d'évaluation qui ne sont pas bien adaptées à l'évaluation des processus d'innovation fondés sur l'apprentissage (Devaux et al., 2008 ; Banque mondiale, 2012).

Le marché dont il est question dans ce type d'approche peut être local, mais il est de plus en plus souvent national (comme dans le cas de Papa Andina), voire international du fait de l'urbanisation et de la mondialisation. De manière générale, les approches par les filières touchent la diversité d'acteurs requise pour que les producteurs, les transformateurs, les intermédiaires et les consommateurs interagissent efficacement et aplanissent toute difficulté (qu'elle soit liée à l'offre ou à la demande) le long de la chaîne de valeur, et ce spécifiquement par l'intermédiaire du marché. Comme d'autres approches faisant intervenir une multiplicité de parties prenantes, beaucoup d'approches par les filières s'intéressent aux questions de mise en place de plates-formes, de facilitation, d'organisation des parties prenantes avec l'aide d'intermédiaires (ou « courtiers ») neutres, et de renforcement des capacités par des subventions de contrepartie. Il reste que l'institutionnalisation ou la viabilité à plus long terme de ces approches s'avère souvent un problème difficile.

Il est intéressant de constater que l'accès équitable des petits agriculteurs au marché devient rapidement une préoccupation centrale pour de nombreuses initiatives SIA dans les pays en voie de développement mais également dans les pays développés. Cette tendance souligne l'importance que beaucoup de pays et d'acteurs, publics ou privés, consentent progressivement à accorder à l'agriculture familiale en tant que contributeur indispensable à un développement économique sain et à long terme, et 
à l'utilisation durable des ressources naturelles, dont les petits producteurs sont à la fois les utilisateurs primaires et les gardiens. Cette évolution montre en outre que les petits agriculteurs travaillent de plus en plus en réponse à la demande des consommateurs qui leur provient par l'entremise des marchés concernant la production ou les produits transformés, et en tenant compte des normes et des considérations de qualité correspondantes.

\section{Développer les partenariats public-privé}

Depuis les années 1990, beaucoup de bailleurs de fonds nationaux et internationaux, dont la Banque mondiale et l'Organisation des Nations unies pour l'alimentation et l'agriculture (FAO), ont donné une place grandissante à l'instauration plus systématique de partenariats public-privé (PPP). Dans un PPP, au moins un organisme public et un organisme privé mettent en commun leurs ressources, leurs connaissances, et les risques encourus pour faire correspondre leurs intérêts respectifs et fournir conjointement des produits et des services innovants. L'objectif de ces PPP est de permettre l'instauration de relations plus efficaces entre des acteurs publics des systèmes de recherche agronomique pour le développement, d'une part, et d'autre part, des acteurs du secteur privé susceptibles de venir compléter l'apport financier public et de mettre leurs compétences entrepreneuriales et leurs liens avec les marchés au service de l'innovation. Si les PPP ont souvent été utilisés jusqu'à présent pour dispenser des services sociaux et environnementaux (tels que l'approvisionnement et le traitement de l'eau potable), il est de plus en plus fait appel à eux pour diffuser l'innovation et l'adoption des technologies (comme dans le cas de la production de sorgho par des petits producteurs kényans pour une brasserie privée, ou la production de viande porcine issue d'une race en voie de disparition pour une entreprise chinoise d'élevage et de transformation de porcs). Les PPP peuvent aider à surmonter les difficultés dues au sous-investissement dans l'innovation agricole et à accélérer le progrès technique en réduisant les risques encourus par les partenaires privés tout en favorisant l'adaptation et la diffusion de nouveaux résultats scientifiques ou de connaissances et de technologies existantes. Un nombre relativement important de ces partenariats ont été créés au cours de la dernière décennie, notamment dans le secteur agricole dans des pays en voie de développement (Hartwich et Tola, 2007 ; Spielman et al., 2009). Des exemples de PPP réussis comprennent des cas où la recherche a su réduire le coût de la transformation de produits bruts, où une amélioration de la qualité des produits a facilité l'accès à des marchés de forte valeur, ainsi que des cas d'échange de matériel végétal de propagation combiné à une externalisation des activités de multiplication des semences à des sociétés privées (Banque mondiale, 2012). Toutefois, afin de garantir un équilibre adéquat entre les intérêts commerciaux des acteurs privés et les intérêts de la société, il convient de bien veiller à ce que des critères encadrant les conditions et la hauteur des interventions du secteur public, ainsi que des principes directeurs, un calendrier et une stratégie de désengagement soient clairement établis (Banque mondiale, 2012).

À l'instar des autres approches, les PPP n'opèrent pas dans le vide : ils sont souvent associés à divers instruments fournissant des mesures incitatives pour favoriser les partenariats (subventions, incubateurs, consortiums, approches participatives des 
chaînes de marchés) et à des approches permettant de limiter les coûts de transaction (dispositifs d'intermédiation, plates-formes) et de traiter les problèmes de droits de la propriété intellectuelle (contrats de licence, bureaux de transfert de technologies).

\section{Créer des espaces multi-acteurs}

Dans la plupart des approches de l'innovation faisant intervenir un ensemble diversifié d'acteurs, des efforts significatifs sont alloués à la création ou au renforcement de plates-formes, de réseaux ou de consortiums d'innovation fonctionnels multiacteurs. Les plates-formes et les réseaux sont considérés comme des moyens de faciliter les interactions nécessaires entre les parties prenantes et d'assurer leur coordination effective en réduisant les coûts de transactions qui seraient sans cela intrinsèquement élevés (Nederlof et al., 2011 ; Hounkonnou et al., 2012).

Les consortiums peuvent être vus comme un cas particulier de plate-forme multiacteurs formalisée réunissant des partenaires et des usagers publics et privés variés autour de problèmes spécifiques et communs nécessitant un investissement dans la recherche-développement. Les membres du consortium définissent conjointement les stratégies correspondantes en matière de recherche-développement, et financent et mettent en œuvre les activités qui en découlent. La plupart des consortiums ont une organisation chef de file, et chaque partenaire joue un rôle particulier et engage des ressources. Les contributions provenant d'une vaste palette d'acteurs, dont des entreprises privées, couvrent plusieurs aspects de la recherche-développement, tels que l'identification de la demande, l'investissement dans la recherche-développement et le transfert et l'adoption de technologies. Les consortiums sont souvent financés par des subventions concurrentielles sur une période limitée (Banque mondiale, 2012). Ces approches ont été appliquées en Australie, au Chili et en Inde, par exemple, où elles se sont révélées capables de réunir une gamme d'acteurs, dont le secteur privé, nécessaires à l'innovation - en couvrant toute l'étendue du spectre de la recherche-développement (encadré 2.3).

\section{Encadré 2.3. Le projet national indien d'innovation agricole (NAIP)}

Le National Agricultural Innovation Project (NAIP), lancé en 2006, s'attache aux problèmes de recherche-développement et d'innovation en modifiant la manière dont les chercheurs, les agriculteurs et les entreprises agricoles interagissent au sein du système national d'innovation agricole. Ce projet, financé par la Banque mondiale, visait à renforcer le rôle du Conseil indien pour la recherche agricole (Indian Council for Agricultural Research ou ICAR) en catalysant et en gérant le changement au sein des systèmes nationaux de recherche agricole, et à promouvoir le développement de trois types de consortiums pluri-acteurs et pluridisciplinaires associant des organismes publics et privés, des universités, des ONG et d'autres participants :

- des consortiums de recherche collaboratifs axés sur le marché orientant leurs travaux sur certaines filières agricoles ;

- des consortiums de recherche sur les moyens d'existence concentrant leurs travaux sur les stratégies susceptibles de fournir des moyens d'existence durables en zone rurale dans environ 110 districts défavorisés ; 
- des consortiums de recherche fondamentale et stratégique plus spécifiquement axés sur des domaines précis de la recherche exploratoire, dont les applications pourraient s'avérer utiles pour certains problèmes agricoles en Inde.

Des consortiums et des alliances de recherche prometteurs ont été financés par le NAIP selon un processus concurrentiel. Dans chaque cas, les membres des consortiums était conjointement responsables de la gouvernance, la conception et la mise en œuvre de leurs programmes de recherche, ainsi que du maintien de dispositifs fiduciaires et de sauvegarde satisfaisants, de la mise en application des innovations produites et de la diffusion des nouvelles connaissances par le biais de conférences, de marchés de l'innovation, de réseaux et de stratégies de communication.

Un bureau d'assistance a été créé afin de soutenir les nouveaux partenariats particulièrement ambitieux que représentent ces consortiums. Sa fonction était d'apporter des conseils pour la préparation des notes d'orientation et des propositions complètes de projets de recherche, pour le choix des partenaires de consortium, et pour la résolution des difficultés de gestion initiales au sein des consortiums. Le bureau d'assistance s'appuyait pour ce faire sur un certain nombre d'outils, et notamment : un portail Internet, des modules d'apprentissage virtuel et multimédia, des bases de données sur les institutions et les organisations potentiellement partenaires, des études de cas sur des projets agricoles mettant en œuvre une approche par les consortiums, une foire aux questions récurrentes (FAQ), des réunions, des ateliers, des analyses et un service de correspondance directe par courriel avec les membres potentiels des consortiums.

Les résultats du NAIP sont nombreux et variés. Ce projet, et l'approche qu'il a mise en œuvre, ont soulevé un intérêt considérable et se sont soldé par le financement de 188 consortiums à ce jour. Le NAIP a su introduire un plus grand pluralisme dans la recherche agricole, $40 \%$ des instituts membres des consortiums étant extérieurs au système ICAR-Universités agricoles publiques. Des partenariats public-privé ont été promus à grande échelle pour la première fois. L'approche par les consortiums a encouragé les synergies, le travail en équipe, le partenariat, la génération de valeur ajoutée, l'apprentissage, une meilleure focalisation des efforts sur les sujets hautement prioritaires, et une recherche de meilleure qualité et plus pertinente. Un impact supérieur est anticipé grâce aux nouvelles technologies et aux produits mis au point dans le cadre des partenariats induits par le NAIP. Enfin, les institutions qui participent aux consortiums ont été renforcées par les programmes de formation et par l'instauration de nouveaux partenariats multi-acteurs. Une illustration en est l'interaction constante entre les secteurs public et privé et les ONG, et la bonne volonté des instituts de l'ICAR à travailler hors de leur domaine habituel. L'ICAR a commencé à généraliser l'approche par les consortiums et le processus de sélection concurrentielle à l'ensemble de ses instituts.

Les problèmes qui restent à régler concernent notamment l'amélioration de la coordination des partenariats, le développement de l'engagement du personnel et de ses aptitudes à nouer des partenariats, les coûts de formation pour les acteurs ou partenaires nouveaux arrivants et l'instauration de procédures efficaces de suivi et d'évaluation'1 (Banque mondiale, 2006, 2012).

1. India: National Agricultural Innovation Project, Project Appraisal Document. Rapport n 34908 -IN, de la Banque mondiale, unité Agriculture et développement rural, Région Asie du Sud, Washington, DC (2006). 
Que ce soit dans le cas des plates-formes, des réseaux ou des consortiums multiacteurs, un sujet récurrent concerne le besoin d'aller au-delà de l'auto-organisation de ces plates-formes et réseaux pour que l'interaction et la collaboration entre les parties prenantes se déroulent sans heurts ni interruptions, par le développement ou la création de fonctions d'intermédiation et de négociation (également appelées courtage ou brokerage) (Perez Perdomo et al., 2010). Le rôle d'intermédiation consiste à prendre le leadership, inciter, catalyser et défendre les intérêts du groupe des parties prenantes en cas de difficultés ou de conflits (voir également l'encadré 2.3 pour le rôle joué par le Bureau d'assistance dans le cas de l'expérience NAIP) et à apporter une aide pour traiter les questions de partage des bénéfices (tels que les droits à la propriété intellectuelle). En réaction à ce qui est de plus en plus considéré comme une lacune importante dans la structure et le fonctionnement habituel des systèmes d'innovation, de nouvelles institutions d'intermédiation peuvent se développer avec comme mission première de remédier à ce manque, comme c'est le cas aux Pays-Bas (Klerkx et Leeuwis, 2008).

\section{Financer l'innovation}

Les initiatives et les approches décrites plus haut nécessitent souvent d'avoir accès à des mécanismes de financement à même d'aider les acteurs concernés à supporter les coûts et frais mis en jeu.

Au nombre des divers mécanismes de financement qui existent (subventions, garanties, capital de risque, incitations fiscales), les allocations ou subventions, et notamment les subventions concurrentielles de recherche, ont fréquemment été utilisées pour promouvoir des activités variées relevant de l'innovation, telles que la recherche tournée vers la demande, la recherche adaptative, les liens recherche-vulgarisationagriculteurs qui améliorent la pertinence et la diffusion des nouvelles technologies, les services tournés vers la demande, les partenariats productifs, et les liens avec les marchés (Banque mondiale, 2010).

On s'est bien plus attaché, jusqu'à présent, à obtenir des fonds pour les institutions de recherche et, dans une moindre mesure, pour les services de conseil agricole et les agriculteurs qu'à appuyer d'autres acteurs et agriculteurs afin qu'ils participent aux processus d'innovation (Banque mondiale, 2010). Cependant, des initiatives SIA tels que NAIP et Papa Andina ont pu bénéficier de l'arrivée de nouveaux mécanismes de financement permettant un meilleur accès aux ressources d'innovation pour une gamme plus large d'acteurs (au point que certains programmes de subvention demandent à ce que plusieurs parties prenantes soumettent une proposition conjointe et se partagent « équitablement» l'allocation entre eux). Les subventions de contrepartie (dans lesquels le montant des fonds alloués est proportionnel au montant des ressources réunies par ailleurs, à partir de leur fonds propres par un ensemble de parties prenantes), souvent sous la forme de fonds nationaux pour l'innovation, gagnent du terrain du fait de leur grande utilité pour promouvoir la génération de technologies pré-commerciales, le transfert et l'adoption de technologies, l'activité économique privée et l'innovation en général, souvent en incluant de nombreuses parties prenantes. En canalisant d'entrée un surcroît d'attention vers la demande et l'utilisation, généralement en attirant les utilisateurs de technologies 
et de connaissances au sein de partenariats, les subventions de contrepartie sont susceptibles de se révéler plus efficaces que les subventions concurrentielles de recherche pour développer l'utilisation de la technologie et des connaissances chez les agriculteurs et les autres entrepreneurs (Banque mondiale, 2010).

Les subventions de contrepartie peuvent cependant poser problème dans certains cas, lorsqu'un grand nombre des parties prenantes ont très peu de ressources propres. Récemment, des mécanismes de financement ont été mis au point à destination des agriculteurs afin de les aider à accéder à l'innovation (voir l'expérience du réseau PROLINNOVA avec le programme Farmer Access to Innovation Resources, Wongtschowski et al., 2010 ; et encadré 2.1). Bien que ce ne soit pas systématique, les subventions aux agriculteurs peuvent comprendre des dispositions pour veiller à ce qu'il soit fait appel à d'autres parties prenantes (chercheurs, services de vulgarisation/conseil agricole, etc.) en tant que prestataires de services (Banque mondiale, 2010 ; Triomphe et al., 2012b).

\section{Promouvoir la commercialisation de la technologie}

Bien que l'approche SIA ne considère pas le transfert de technologies en soi comme un outil suffisant pour aiguillonner l'innovation, la capacité à gérer les mécanismes formels de transfert de technologies reste bien souvent un atout déterminant, par exemple pour s'engager efficacement dans des PPP et, de plus en plus, pour transférer des technologies susceptibles d'être diffusées par les circuits de commercialisation.

Les bureaux de transfert de technologies (Technology Transfer Offices ou TTO), très courants dans les pays développés, sont des unités spécialisées affiliées à un organisme de recherche ou à une université et qui ont pour fonction d'identifier et de protéger les résultats des recherches, ainsi que de faciliter leur application et leur commercialisation. De tels bureaux sont de plus en plus créés dans les pays en voie de développement pour diversifier les sources de financement, inciter le personnel du secteur public à se lancer dans des activités commerciales, et commercialiser les technologies. Les TTO sont à même de faire reconnaître plus largement le travail de l'organisme de recherche dont ils dépendent (renforçant au passage la perception de sa valeur dans la population), de transférer les technologies vers les utilisateurs finaux (semenciers ou agriculteurs, par exemple) et de générer des revenus pour financer la poursuite des recherches. Ils peuvent également fournir une expertise spécialisée sur la protection de la propriété intellectuelle et/ou sur les accords juridiques et contribuer au transfert officiel de technologies depuis des organismes publics, des universités ou le secteur privé vers des partenaires commerciaux ou internationaux.

Certains bureaux de transfert de technologies hébergent en outre des incubateurs afin d'aider les entreprises axées sur la technologie (souvent créées par des chercheurs) à commercialiser leurs produits innovants. L'instauration de liens solides et intimes entre la recherche et les entreprises, et la création d'un environnement porteur à même de favoriser et d'accompagner leur essor sont au centre du mode de fonctionnement des incubateurs. Ils fournissent une assistance pratique en matière de gestion, un accès à des équipements et à des services de financement, d'appui technique et commerciaux, et des locaux communs (Banque mondiale, 2012). 
L'Institut international de recherche sur les cultures des zones tropicales semi-arides (ICRISAT) a ainsi créé un incubateur qui commercialise des technologies mises au point par les chercheurs indiens à destination des petits agriculteurs. La plupart des incubateurs se donnent toutefois une mission plus globale d'appui à l'innovation provenant de petites et moyennes entreprises, par exemple au développement des entreprises agro-alimentaires en général.

Les pôles scientifiques (également appelés pôles technologiques, pôles de recherche, technopôles ou pôles d'excellence) sont des organisations dont l'objectif principal est d'accroître la prospérité locale en favorisant une culture d'innovation et en améliorant la compétitivité des entreprises et des institutions locales fondées sur le savoir. Ils y parviennent en stimulant et en gérant le flux de connaissances et de technologies qui circule entre les parties prenantes concernées, en exploitant les processus d'incubation et les effets d'entraînement, et en fournissant d'autres services à valeur ajoutée ainsi qu'un espace et des installations de haute qualité. Les pôles scientifiques sont utiles pour favoriser les PPP dans des contextes d'innovation plus matures. Ils fonctionnent de manière optimale lorsqu'ils disposent de capitaux d'investissement du secteur privé, d'une expertise technique industrielle, et d'une base de connaissances et de technologies suffisamment solide. Ils constituent un lien utile entre le secteur privé et les instituts de recherche, en particulier les universités, en introduisant sur le marché des produits prometteurs issus de la science et en fournissant un appui pour la modification des produits. Les diverses fonctions assurées par les pôles scientifiques comprennent la facilitation de partenariats publicprivé pour la recherche, la mise à disposition d'infrastructures, ainsi que des services variés, tels que l'aide aux entreprises (Banque mondiale, 2012).

\section{W Défis rencontrés dans la mise en œuvre des systèmes d'innovation agricole}

La mise en œuvre des approches et des instruments décrits plus haut a d'ores et déjà contribué de manière positive au développement de l'innovation, mais plusieurs défis importants restent à relever.

Une première difficulté est la complexité même du travail de compréhension et d'évaluation et - peut-être plus important encore - de celui de conception et de pilotage de la mise en place de dispositifs SIA. Comme le Centre technique de coopération agricole et rurale (CTA) l'a fait remarquer au cours du colloque ISDA, « il est difficile pour la plupart des chercheurs de bien comprendre le concept de système d'innovation, qui est conceptuellement diffus, enraciné dans la théorie économique et passablement éloigné de leur formation disciplinaire ». Même des « courtiers en innovation » et des « coachs en innovation » avec des années d'expérience peuvent peiner à bien comprendre les bases de la pensée des systèmes d'innovation (Pyburn et Woodhill, 2011). La raison en est que les publications consacrées à ce sujet sont en grande partie rédigées en employant un langage et des notions plutôt abstraits et souvent vagues (empruntant des termes tels que réseaux, plates-formes, et innovation institutionnelle, pour n'en citer que quelques-uns) qui relèvent des sciences sociales et qui sont relativement difficiles à expliquer, réclamant d'entrée un certain 
bagage théorique. Qui plus est, même lorsque les concepts sont acquis, il reste à rendre opérationnels la pensée et l'approche SIA - ce qui reste encore limité.

Une autre difficulté réside dans la ténuité du lien de causalité qui existe entre les approches et les instruments potentiellement utiles (tels que ceux décrits plus haut) qui sont appliqués, d'une part, et le résultat obtenu en matière d'innovation, d'autre part. En d'autres termes, l'issue et la direction d'un processus d'innovation ne dépend pas seulement des approches employées ; elles dépendent également, en grande partie, de facteurs et de conditions qui relèvent du fameux mais flou " environnement porteur », qui varie considérablement, par exemple entre des contextes plus agraires ou plus axés sur les marchés, ainsi qu'au sein d'un même pays (Banque mondiale, 2012). Elles sont également influencées par le cadre politique et fiscal, généralement complexe, régissant les aspects scientifiques, technologiques, juridiques, consultatifs et commerciaux, qui tous affectent l'innovation par des voies, tant directes qu'indirectes.

Les approches SIA promeuvent fermement un changement dans la manière d'aborder l'innovation. Cependant, le passage à une échelle supérieure et l'institutionnalisation, en ce qui concerne non seulement les résultats (le notoire quoique difficile à cerner « transfert de technologies »), mais aussi les approches novatrices de l'innovation elles-mêmes, sont une tâche particulièrement ardue, dans la mesure où il peut s'avérer nécessaire de changer la manière dont des institutions et des bureaucraties entières opèrent habituellement - ce qui prend du temps. Pour y parvenir, il convient d'investir dans l'apprentissage et dans le renforcement des capacités (voir plus loin), de fournir des mesures incitatives permettant aux acteurs de mettre en œuvre de nouvelles compétences, et de modifier les cadres réglementaires ou politiques inadéquats. Il est également important d'encourager de nouvelles attitudes et de nouveaux comportements tels que l'ouverture d'esprit, la flexibilité et la capacité d'adaptation. Pour l'exprimer brièvement, le passage à une échelle supérieure et l'institutionnalisation nécessitent que l'on change une partie de « l'environnement porteur » mentionné plus haut.

De tels changements lourds de conséquences appellent la création et le renforcement d'un capital humain suffisant pour satisfaire les besoins des diverses parties prenantes. Plusieurs principes de base ont été dégagés pour pouvoir progresser dans ce sens :

- l'instauration de nouveaux programmes d'enseignement plus stratégiquement en prise avec les besoins des acteurs sociaux et productifs ;

- la mise au point de nouveaux cursus d'enseignement, qui confèrent la capacité de faire face à la complexité, au changement, et aux processus multi-acteurs dans le cadre de l'innovation rurale, tout en permettant une plus grande spécialisation ;

- et le développement des facultés d'innovation des organisations et des individus intervenant dans l'enseignement et la formation agricoles.

De plus, les sommes qui sont ainsi à investir pour le renforcement des capacités sont considérables et ne peuvent être allouées du jour au lendemain. En outre, certaines parties prenantes, et notamment les plus pauvres, peuvent ne pas pouvoir accéder facilement à ces services de renforcement des capacités.

Bien que peu d'auteurs abordent cet aspect de manière explicite, l'application pratique de l'approche par les SIA met en évidence les difficultés qu'il y a à traiter 
concrètement les fortes asymétries en matière de pouvoir, de ressources et de capacités qui peuvent exister entre les parties prenantes, et qui ont souvent par ailleurs des dimensions politiques sous-jacentes. De telles asymétries sont chose courante dans les dispositifs d'innovation dans la plupart des pays en voie de développement (Hocdé et al., 2009 ; Faure et al., 2010). À quel point les initiatives SIA sont-elles en mesure de contribuer concrètement à réduire ces asymétries, par exemple en renforçant la voix, les capacités et l'autonomie des petits agriculteurs et des autres acteurs ruraux désavantagés, est une question âprement débattue. Les expériences PROLINNOVA (encadré 2.1) et Papa Andina (encadré 2.2) mettent explicitement en avant des actions en faveur des plus pauvres dans leur manière de susciter l'innovation. En dépit d'exemples encourageants tels que ceux-ci, obtenir que des parties prenantes aussi défavorisées puissent interagir de manière pertinente et équitable avec celles qui sont plus puissantes ou mieux organisées, et que ce faisant elles soient effectivement en mesure de tirer avantage de la dynamique d'innovation, n'est malheureusement ni automatique ni facile. Les membres de l'establishment des institutions de recherche, de vulgarisation et d'enseignement agricoles - bien souvent le personnel des niveaux intermédiaires des organismes publics - tendent à s'offusquer et à s'opposer à l'idée de devoir partager leur pouvoir et leurs ressources, parce qu'ils craignent de perdre le contrôle et parce qu'ils ne savent pas quoi penser des nouveaux rôles qui leur sont dévolus. De leur côté, les agriculteurs aussi éprouvent des difficultés à assumer de nouveaux rôles. Des dispositifs efficaces d'intermédiation et de renforcement des capacités des parties prenantes les plus modestes peuvent contribuer à atténuer les asymétries, mais la résolution des problèmes de ce type, si et quand la chose est possible, dépend bien plus souvent des évolutions à long terme et d'interventions qui dépassent largement le cadre des initiatives SIA. Ceci conduit à s'interroger sur l'horizon temporel de beaucoup d'interventions d'innovation, en particulier de celles qui sont mises en œuvre dans le cadre de projets à financement extérieur de courte durée, dont les effets positifs transitoires, obtenus dans des conditions de ressources et de règles du jeu artificialisées, tendent à s'estomper dès l'achèvement du projet.

Il convient enfin de mentionner les difficultés qui sont liées au suivi et à l'évaluation appropriés de l'impact des approches, des instruments et des investissements en matière de SIA (UE SCAR, 2012). Étant donné le caractère novateur de ces approches et les attentes qui s'y attachent quant à leur capacité à générer une innovation plus pertinente, il apparaît primordial de se donner les moyens d'évaluer leur impact réel et de comparer entre elles différentes initiatives les mettant en pratique. Cependant, de nombreuses expériences en cours sur les systèmes d'innovation mettent en évidence la complexité de cette tâche, en raison de la multiplicité des dimensions à surveiller et du fait que les indicateurs nécessaires pour les décrire de manière satisfaisante dans le temps et l'espace en tenant compte de la diversité des parties prenantes et des activités mises en jeu exigeraient théoriquement l'estimation d'un très grand nombre de variables quantitatives et qualitatives dont la signification et l'interprétation pourraient ne pas s'avérer simple. Le coût d'un tel suivi est en lui-même un problème, car peu de parties prenantes ou d'organismes de financement sont disposés, ou même simplement capables, d'investir les sommes nécessaires à la mise en place d'un système performant, et donc assez coûteux, de suivi et d'évaluation. 


\section{W Conclusions et perspectives}

Au cours de ce bref tour d'horizon, nous avons discuté de la signification et de l'intérêt de l'approche émergente par les systèmes d'innovation, ainsi que d'approches et instruments avec lesquels elle peut être appliquée dans le secteur agricole, et nous avons présenté quelques uns des problèmes qu'elle pose. Les approches SIA actuelles découlent clairement des enseignements qui ont pu être tirés des approches et paradigmes précédents, tels que le renforcement des systèmes nationaux de recherche agricole et des systèmes d'information et de connaissances agricoles. Les investissements de base dans les infrastructures et l'équipement de recherchedéveloppement, dans les ressources humaines et dans la performance et la responsabilisation du système restent des fondamentaux indispensables auxquels l'approche SIA peut venir ajouter des facettes complémentaires, telles qu'une place particulière accordée à la diversité des parties prenantes et à leurs interactions, aux compétences, et aux liens avec les marchés, le tout en accordant une attention soutenue aux aspects politiques et à l'environnement porteur général.

Il est encore tôt pour évaluer l'impact global de l'approche SIA sur la dynamisation de l'innovation et au-delà sur ses effets, notamment pour comparer l'utilité de cette approche à celle des paradigmes antérieurs, encore très présents, tels que le modèle linéaire de la mise au point et du transfert des technologies. Toutefois, l'attractivité intrinsèque des SIA est forte et, de plus en plus, les acteurs et les organisations, les institutions, et les décideurs, tant dans les pays développés que dans les pays en voie de développement, sont en train d'investir dans des projets et des initiatives à plus grande échelle pour tenter de stimuler l'innovation.

Il est certain que le chemin ne sera pas facile, et l'approche SIA, quel que soit son potentiel intrinsèque, n'est certes pas la panacée qui garantira que les besoins d'innovation dans le domaine du développement agricole et rural, protéiformes et insaisissables, seront toujours pris en compte de manière satisfaisante, dans toutes les situations.

Au stade où nous nous trouvons actuellement, des efforts doivent être fournis dans plusieurs directions pour veiller à ce que l'approche par les SIA puisse déployer tout son potentiel et produire les bénéfices escomptés :

- Faire mieux connaître le potentiel de l'approche SIA auprès des décideurs et des investisseurs. Le manuel consacré aux SIA par la Banque mondiale et ses partenaires représente une tentative dans ce sens, mais des efforts similaires s'appuyant sur des éléments plus diversifiés et mieux ciblés pourraient s'avérer utiles pour faire connaître les fondements de cette approche à d'autres décideurs et investisseurs dans le monde et leur présenter des preuves convaincantes, provenant d'expériences pilotes bien choisies, de son impact. Un des objectifs sera de stimuler la volonté politique et d'apporter des arguments pertinents aux ministères, aux institutions publiques et au secteur privé pour qu'ils approuvent et adoptent un mode d'action efficace fondé sur les systèmes d'innovation.

- Mettre au point des mécanismes de financement de l'innovation qui soient appropriés et solidement nantis. En se basant sur les expériences passées, les mécanismes de financement doivent prendre en compte les dimensions multiples des approches SIA et la dynamique sous-jacente des processus d'innovation réels. Ils 
doivent permettre à des acteurs divers d'avoir un accès souple aux ressources dont ils ont besoin pour s'inscrire dans le jeu dynamique des innovations, à l'échelle qui leur convient, en prise avec les besoins évolutifs des utilisateurs et réactifs aux changements incessants qui affectent l'environnement général et local.

- Constituer une communauté de pratiques sur les SIA. Les efforts en matière de SIA sont encore rares et dispersés, d'où la relative lenteur observée dans les progrès de la compréhension de ces initiatives et dans l'exploitation des expériences passées et en cours dans ce domaine. Alors que les universités agricoles finiront par rattraper leur retard en ce qui concerne l'enseignement des concepts liés aux systèmes d'innovation aux nouvelles générations d'étudiants, l'instauration d'une communauté de pratique active sur les SIA pourrait constituer un bon moyen de dynamiser l'apprentissage collectif et l'expertise en matière d'expériences de SIA, et de développer plus avant les approches et les instruments nécessaires pour réussir leur application dans des contextes variés.

- Mieux comprendre l'approche des SIA, ses implications et son impact. Bien que l'approche SIA soit progressivement en train de développer un cadre opérationnel, elle demeure un concept et une pratique en évolution, qui réclame une meilleure compréhension et une analyse plus approfondie. Il convient en outre d'accorder plus d'importance à l'évaluation des initiatives SIA et de leur impact, ce qui exige de créer des capacités, des outils et des méthodes spécifiques et adaptées, et d'identifier des indicateurs qui pourraient permettre de comparer de manière pertinente et instructive les diverses expériences SIA mises en œuvre aux niveaux national et international.

À des degrés divers, nombre de ces recommandations sont déjà suivies. Ces efforts fournissent progressivement les résultats nécessaires pour permettre une application de l'approche SIA qui soit rigoureuse, réfléchie, souple et soucieuse du contexte. Ce faisant, il convient d'œuvrer sans cesse à ce que l'approche SIA et les autres approches et instruments qui lui sont associés restent suffisamment flexibles, diversifiés et adaptables pour permettre leur application dans l'environnement particulier que l'on désire cibler. Ne pas y parvenir et laisser l'approche SIA devenir un modèle rigide, mécanique et prétendument universellement applicable en l'état pour induire l’innovation, serait le chemin le plus court vers les désillusions.

\section{" Références bibliographiques}

Akrich M., Callon M., Latour B., 1988. À quoi tient le succès des innovations. Premier épisode : l'art de l'intéressement, Deuxième épisode : l'art de choisir les bons porte-parole. Gérer et comprendre. Annales des Mines, 11, 4-17 ; 12, 14-29.

Albaladejo C., Casabianca F., 1997. La recherche-action. Ambitions, pratiques, débats. Inra - SAD. Études et Recherches sur les systèmes Agraires et le Développement n³0.

Alter N., 2000. La trajectoire des innovations, In : L'innovation ordinaire, PUF, Paris, 7-39.

Ashby J., Sperling L., 1995. Institutionalizing participatory, client-driven research and technology development in agriculture. Development and Change, 26, 753-770.

Banque mondiale, 2006. Enhancing agricultural innovation: How to go beyond the strengthening of research systems, World Bank, Washington.

Banque mondiale, 2010. Designing and implementing agricultural innovation funds: Lessons from competitive and matching grant projects, Washington. 
Banque mondiale, 2012. Agricultural innovation systems. An investment sourcebook, World Bank, Washington, $658 \mathrm{p}$.

Benor D., Baxter M., 1984. Training and visit extension. Washington, World Bank and International Bank for Reconstruction and Development, $202 \mathrm{p}$.

Béguin P., 2003. Design as a mutual learning process between users and designers. Interacting with computers, 15 (5), 709-730.

Biggs S., Smith G., 1998. Beyond methodologies: Coalition-building for Participatory Technology Development. World Development, 26(2), 239-248.

Byerlee D., Alex G.E., 1998. Strengthening national agricultural research systems. Policy issues and good practice. The World Bank, Washington, $88 \mathrm{p}$.

Callon M., Lascoumes P., Barthe Y., 2001. Acting in an uncertain world. An essay on technical democracy, The Mit Press, Cambridge, Massachusetts, London, England (English translation 2009).

Chambers R., Pacey A., Thrupp L.A., 1989. Farmer first. Farmer innovation and agricultural research, London, Intermediate Technology Publication.

Devaux A., Horton D., Velasco C., Thiele G., Lopez G., Bernet T., Reinoso I., Ordinola M., 2009. Collective action for market chain innovation in the Andes. Food Policy, 34 (1), 31-38.

Evenson R.E., Gollin D., 2003. Assessing the impact of the green revolution, 1960 to 2000. Science, 300 (5620), 758-762.

Faure G., Gasselin P., Triomphe B., Temple L., Hocdé H., 2010. Innover avec les acteurs du monde rural. La recherche-action en partenariat. Collection «Agricultures tropicales en poche », Éditions Quae - CTA - Presses Agronomiques de Gembloux, 222 p.

Freeman C., 1988. Japan: a new national system of innovation? Technical change and economic theory, Pinter, London.

Fukuda C., Watanabe C., 2008. Japanese and US perspectives on the National Innovation Ecosystem. Technology in Society, 30 (1), 49-63.

Hagmman J., Chuma E., Murwira K., 1996. Improving the output of agricultural extension and research through participatory innovation development and extension; experiences from Zimbabwe. European Journal of Agricultural Education and Extension, 2 (4), 15-23.

Hall A., Sulaiman V.R., Clark N., Yogoband B., 2003. From measuring impact to learning institutional lessons: an innovation systems perspective on improving the management of international agricultural research. Agricultural Systems, 78, 213-241.

Hartwich F., Tola J., 2007. Public-private partnerships for agricultural innovation: concepts and experiences from 124 cases in Latin America. Int. J. Agric. Resources, Governance and Ecology, 6 (2), 240-255.

Hocdé H., Triomphe B., Faure G., Dulcire M., 2009. From participation to partnership, a different way for researchers to accompany innovations processes: challenges and difficultie, In: Innovation Africa: enriching farmers' livelihoods (Sanginga P., Waters-Bayer A., Kaaria S., Njuki J., Wettasinha C., eds), Earthscan, London.

Hounkonnou D., Kossou D., Kuyper T.W., Leeuwis, C., Nederlof S., Röling N., Sakyi-Dawson O., Traoré M., van Huis A., 2012. An innovation systems approach to institutional change: Smallholder development in West Africa. Agricultural Systems, 108, 74-83.

Hussain S.S., Byerlee D., Heisey P.W., 1994. Impacts of the training and visit extension system on farmers' knowledge and adoption of technology: Evidence from Pakistan. Agricultural economics, 10 (1), 39-47.

Jouve P., Mercoiret M.-R., 1987. La recherche développement : une démarche pour mettre les recherches sur les systèmes de production au service du développement rural. Les Cahiers de la Recherche-Développement, 16, 8-13.

Klerkx L., Leeuwis C., 2008. Matching demand and supply in the agricultural knowledge infrastructure: experiences with innovation intermediaries. Food Policy, 33, 260-276.

Knickel K., Tisenkopfs T., Peter S., 2009. Innovation processes in agriculture and rural development. Results of a cross-national analysis of the situation in seven countries, research gaps and recommendations. In-Sight project report. 
Latour B., 1987. Science in action: How to follow scientists and engineers through society (Milton Keynes: Open University Press).

Lewin K., 1946. Action research and minority problems. Journal of Social Issues, 2, 34-46.

Liu M., 1997. Fondements et pratiques de la recherche-action, L'Harmattan, Paris, 350 p.

Lundvall B.A., 1992. National Systems of Innovation, Pinter, London.

Metcalfe J.S., 1995. The economic foundations of technology policy, In : Handbook of the economics of innovation and technological change (Stoneman P., ed.), Oxford University Press.

Nederlof S, Wongtschowski M., ven der Lee F., 2011. Putting heads together. Agricultural innovation platforms in practice, Bulletin 396, KIT Publishers, Amsterdam, $192 \mathrm{p}$.

Nelson R., 1993. National innovation systems: A comparative analysis, University Press, Oxford, New York.

Norman D., Collinson M., 1985. Farming systems research in theory and practice, In : Agricultural systems research for developing countries, Richmond, Australia, 12-15 mai 1985, ACIAR, No 11, 16-30.

OCDE, 1997. National Innovation Systems, Organization of Economic Cooperation and Development, Paris.

Perez Perdomo S., Klerkx L., Leeuwis C., 2010. Innovation brokers and their roles in value chainnetwork innovation: preliminary findings and a research agenda, In : Innovation and sustainable development in agriculture and food (ISDA), Montpellier, France.

Pyburn R., Woodhill J., 2011. Dynamics of rural innovation: A primer for emerging professionals, KIT Publishers, Amsterdam, 128 p.

Rajalahti R., Woelcke J., Pehu E., 2005. Development of research systems to support the changing agricultural sector, In : Proceedings. Agriculture and rural development Discussion Paper 14, World Bank, Washington, DC.

Rajalahti R., Janssen W., Pehu E., 2008. Agricultural innovation systems: from diagnostics toward operational practices, Discussion Paper 38, Washington, World Bank, 105 p.

Rogers E.M., 1983. Diffusion of innovations, New York, Free Press (third edition).

Röling N., 1990. The agricultural research-technology transfer interface: A knowledge systems perspective, In : Making the link; agricultural research and technology transfer in developing countries (Kaimowitz D., ed.), Westview Press, ISNAR. 1-4, 11-23.

Schumpeter J., 1942. Capitalism, socialism, and democracy, Harper and Row, New York.

Spielman D.J., Hartwich F., Grebmer K., 2009. Public-private partnerships and developing-country agriculture: Evidence from the international agricultural research system. Public Administration and Development, 30 (4), 261-276.

Triomphe B., 2012a. Codesigning innovations: How can research engage with multiple stakeholders? In : Agricultural innovation systems. An investment sourcebook, World Bank, Washington, 308-315.

Triomphe B., Wongtschowski M., Krone A., Waters-Bayer A., Lugg D., van Veldhuizen L., 2012b. Providing farmers with direct access to innovation funds, In: Agricultural innovation systems. An investment sourcebook, World Bank, Washington, 435-441.

UE SCAR, 2012. Agricultural knowledge and innovations systems in transition. A reflection paper, Standing Committee on Agricultural Research, Brussels, 117 p.

Veldhuizen L., Waters-Bayer A., de Zeeuw H., 1997. Developing technology with farmers. ETC Netherlands and Zez Books, 1997.

Wongtschowski M., Triomphe B., Krone A., Waters-Bayer A., van Veldhuizen L., 2010. Towards a farmer-governed approach to agricultural research for development: Lessons from international experiences with local innovation support funds, In : Innovation and sustainable development in agriculture and food (ISDA), Montpellier, France - http://hal.archives-ouvertes.fr/hal-00510417/fr/ 


\title{
Chapitre 3 \\ De quels types d'entrepreneurs innovants avons-nous besoin?
}

\author{
Andy Hall et Kumuda Dorai
}

L'innovation agricole fait invariablement intervenir toute une gamme de partenariats, d'alliances et de dispositifs de type réseaux qui relient entre eux les utilisateurs de connaissances, les producteurs de connaissances et les autres acteurs qui contribuent à rendre possible l'innovation au niveau des marchés, des politiques et de la société civile. Il existe aujourd'hui une vaste littérature conceptuelle et empirique qui voit dans l'innovation agricole non pas tant un processus d'invention tiré par la recherche qu'un processus consistant à exploiter des idées (anciennes et nouvelles) d'une manière innovante, dans l'intention bien précise de produire une valeur ajoutée sociale, économique et/ou environnementale (Juma, 2010).

Ce n'est qu'au cours des 20 dernières années environ que les cadres politiques ont progressivement accepté de reconnaître ce qui avait déjà été depuis longtemps établi dans la littérature : l'innovation agricole émerge de l'interaction entre une multiplicité d'acteurs. Cette position est reflétée par l'idée, largement admise, selon laquelle la recherche agricole se conçoit moins comme une activité isolée et autonome que comme faisant partie intégrante d'un système de connaissances agricoles ou, plus récemment, d'un système d'innovation agricole (Banque mondiale, 2006).

Ce qui devient de plus en plus significatif, c'est que l'éventail des acteurs impliqués dans ces partenariats dépasse aujourd'hui le cercle des acteurs classiques des secteurs publics et privés, des ONG et de la recherche, et va jusqu'à inclure un nouvel ensemble d'intervenants qui surprennent souvent la manière de voir conventionnelle et brouillent les lignes, ou endossent des rôles multiples en poursuivant leurs missions pour le bien social et environnemental, tout en surveillant les marges de profitabilité.

Cette nouvelle classe d'entrepreneurs, bien souvent dans la zone d'ombre du $\operatorname{radar}^{1}$ aux yeux du marché et des politiques, est en train d'explorer de nouveaux

1. Ces opérations sont qualifiées comme étant dans « la zone d'ombre du radar » du fait qu'elles sont ignorées ou négligées, non seulement par les décideurs mais également par les acteurs conventionnels du secteur privé, dans le paysage général de l'innovation, tout en étant de nature à apporter beaucoup pour le développement de ce dernier. 
modes - perturbateurs - d'innovation qui répondent aux préoccupations sociales et environnementales que les politiques publiques ne savent par quel bout attaquer. L'accent placé sur le secteur privé - en présumant qu'il s'agit là d'entreprises - dans l'approche par les systèmes d'innovation a masqué l'importance d'autres formes d'entrepreneuriat. Beaucoup de ces dernières ont une longue expérience du développement. Peut-être est-il temps de commencer à regarder les zones d'ombre du radar et à aider les entrepreneurs qui s'y trouvent.

Nous vivons une période déroutante pour ceux qui aiment classer les organisations en catégories confortablement familières et bien étanches. Considérons par exemple le Bangladesh Rural Advancement Committee (le Comité du Bangladesh pour le progrès rural), mieux connu sous l'acronyme de BRAC. Il s'agit d'une organisation non gouvernementale dont la mission consiste à « renforcer les moyens d'action des personnes et des communautés en situation de pauvreté, d'analphabétisme, de maladie et d'injustice sociale ». Mais cette ONG est par ailleurs un acteur majeur des filières, intervenant à la fois au niveau de l'approvisionnement en intrants et de la transformation agroalimentaire. Elle concurrence sérieusement les agro-industries privées, et pourtant elle répond également aux besoins de sa clientèle démunie. Elle revendique l'entrepreneuriat social comme une de ses stratégies maîtresses. Amul, une célèbre coopérative laitière indienne, en constitue un autre exemple. Propriété conjointe de 3,03 millions de producteurs laitiers, elle collecte le lait auprès des éleveurs, le transforme en divers produits qu'elle commercialise, en Inde comme à l'étranger. Amul assure en parallèle le service de fourniture des intrants nécessaires à ses producteurs et offre des formations pour développer les compétences.

Le BRAC et Amul ne sont que deux exemples parmi une foule d'acteurs similaires qui se profilent peu à peu comme des intervenants clés dans le secteur du développement, que les politiques tendent très généralement à ne pas voir, et que la littérature en matière de développement agricole commence tout juste à prendre en compte (encadré 3.1).

\section{Encadré 3.1. Quelques exemples d'esprit d'entreprise \\ Real IPM (Kenya)}

Real IPM est une entreprise privée kenyane qui tire profit de la production et de la vente de bio-pesticides pour le secteur de l'horticulture commerciale en Afrique de l'Est. De philosophie profondément écologique, elle a récemment investi un nouveau marché de taille : les paysans pauvres. En collaboration avec le programme RIU (Research Into Use) du DFID (Department for International Development - ministère britannique du développement international), elle a mis en place un réseau de conseillers dans les villages pour contribuer à promouvoir et à vendre auprès des petits agriculteurs un bio-pesticide contre la striga, une mauvaise herbe parasite du maïs, la culture vivrière de base. L'entreprise a dû franchir un certain nombre d'obstacles en matière de réglementation pour pouvoir homologuer ses produits en vue de leur usage commercial - la législation prévoyait en effet des autorisations pour les pesticides de synthèse, mais pas pour les agents de bio-lutte. Real IPM a 
été forcé de se consacrer à des activités de diffusion des connaissances et de négociation auprès des décideurs, notamment en allant au-devant des pouvoirs publics et des chercheurs, en présentant des preuves scientifiques obtenues par des essais en champs et en cultivant des relations avec diverses parties prenantes du processus de réglementation qui se montraient intéressées. Ce travail a entraîné une révision des réglementations concernant les importations et a suscité la rédaction d'une version préliminaire de la réglementation kenyane en matière d'intrants pour l'agriculture biologique - bien que l'agent de bio-lutte attende encore son autorisation de mise sur le marché.

\section{FIPS (Kenya)}

Farm Input Promotion Services (FIPS) est une entreprise qui fait la promotion de semences et d'engrais en petits conditionnements à destination des paysans pauvres au Kenya. Dans certaines régions rurales du pays, le taux d'adoption des engrais et des semences améliorées reste bas du fait du prix élevé des intrants, seulement disponibles en gros conditionnements. Beaucoup de zones rurales où la demande est faible sont mal desservies par les fournisseurs d'intrants privés. Pour tenter de résoudre ce problème, FIPS a donné un premier coup de pouce à la demande en négociant la fourniture de semences et d'engrais en petits volumes, moins chers à l'achat, auprès d'entreprises productrices d'intrants. FIPS apporte alors des conseils techniques aux agriculteurs pour les encourager à utiliser ces intrants. Pour y parvenir, FIPS joue le rôle d'intermédiaire pour mettre en contact, par des réseaux, les paysans et les distributeurs locaux d'intrants, les organismes de recherche publics et les fabricants d'intrants. Fournir aux agriculteurs un accès à la technologie était l'idée de départ, mais FIPS remplit désormais une fonction de tisseur de liens avec les systèmes d'approvisionnement locaux et avec la recherche.

TerraCycle Inc. (USA)

TerraCycle est une entreprise privée américaine spécialisée dans la fabrication de produits de consommation à partir de rebuts difficiles à recycler. Après avoir commencé en produisant un engrais à base de déchets organiques traités par lombricompostage, l'entreprise a diversifié sa gamme pour proposer des sacs, des bancs publics et des pots de fleur, à partir du recyclage de matériaux utilisés pour le conditionnement de la production d'autres entreprises.

\section{Gram Mooligai Company Ltd. (Inde)}

Gram Mooligai est une société anonyme de droit public spécialisée dans la collecte, la culture, la transformation et la commercialisation de plantes médicinales et de miel pour l'exportation. Elle appartient, par répartition équitable des bénéfices, à plusieurs groupes d'entraide de femmes réunissant plus de 1000 familles dans le sud de l'Inde. Cette société, comme la fondation qui l'a créée à partir de fonds de donateurs, consacre également une partie de ses ressources à former les agriculteurs aux pratiques de l'agriculture biologique.

Ces types d'organisation ouvrent-elles de nouvelles possibilités de renforcer les capacités d'innovation à une époque où l'idée de mettre en place des systèmes d'innovation favorables aux pauvres commence à s'enliser ? Pour répondre à cette question, il est utile de revenir en arrière et de regarder ce que le concept de système d'innovation avait à offrir et pourquoi les rouages se sont grippés. 


\section{W Les systèmes d'innovation : au-delà des concepts ?}

Une caractéristique intéressante du débat autour de la nature de l'innovation agricole dans les pays en voie de développement a été l'idée partagée selon laquelle il s'agit là d'un processus enchâssé dans un ensemble bien plus large de relations que celles que laisse entendre l'enchaînement recherche-vulgarisation-agriculteur. Biggs et Clay (1981) et Biggs (1990) ont évoqué des sources diverses et multiples d'innovation. Röling (1992) a introduit la notion de systèmes de connaissances agricoles et la Wageningen School of Innovation Studies a eu recours à ce type de concept pour explorer les paysages de l'innovation rurale multi-acteurs (Engel, 1995 ; Leeuwis et Pyburn, 2002). Deux débats ont émergé parallèlement l'un à l'autre, renforçant cette perspective multi-acteur. D'une part, dès les années 1980, une tendance à réexaminer le rôle de l'État dans les économies nationales s'est répandue. Cette idée est partie d'Europe et d'Amérique du Nord pour parvenir aux économies émergentes d'Asie du Sud et d'Afrique dans le sillage de l'aide au développement et des conditionnalités liées aux investissements des banques de développement - plus communément appelées ajustements structurels. D'autre part, l'importance du secteur privé dans l'innovation a été progressivement révélée, montrant bien que l'innovation n'était pas exclusivement liée aux activités publiques de recherche-développement (ni d'ailleurs aux activités de recherche-développement en général) mais qu'elle était répartie dans l'ensemble de l'économie. Les pays qui s'en sortaient le mieux se sont avérés être ceux dans lesquels un système national d'innovation était étayé par un réseau dense d'interactions (Freeman, 1995).

Le concept de système national d'innovation a progressivement été adapté pour étudier les processus d'innovation dans le cadre du développement agricole (Hall et al., 1998, 2002 ; Hall, 2007). Ce travail découle des travaux de Biggs, Röling et d'autres, mais est bien plus explicite quant à l'importance des acteurs du secteur privé dans le processus d'innovation agricole, et souligne le fait qu'un macroenvironnement porteur est tout aussi déterminant que les activités de microinnovation des agriculteurs dans leur espace rural (Banque mondiale, 2006). Certaines des premières études sur le sujet ont prédit, un peu prématurément, que les modèles d'innovation qui dépendaient ainsi du secteur privé pouvaient potentiellement se montrer à la hauteur d'objectifs de développement tels que la réduction de la pauvreté (Hall et al., 2002). Depuis, les publications sur les systèmes d'innovation agricole ont couvert une gamme élargie de formules d'innovation - certaines plus participatives, d'autres plus liées à la recherche (Hall, 2009). L'ensemble de ces réflexions soulignent bien le rôle majeur du secteur privé dans divers types d'activités d'innovation et à différents moments de la trajectoire d'innovation (Hall, 2006, 2009).

La plus grande contribution de l'idée de système d'innovation au développement agricole et rural est de nature conceptuelle. Elle a aidé les planificateurs à ré-examiner la place de la recherche agronomique au sein des processus dynamiques d'innovation, et elle a mis en évidence l'importance des interrelations qui permettent de faire le lien entre la recherche et les autres acteurs du processus. Toutefois, le concept de système d'innovation ne constitue pas un nouveau modèle sur lequel calquer l'innovation, mais une métaphore expliquant l'immense diversité 
des manières d'organiser l'innovation à différentes fins et dans différents contextes. Mais comment appliquer son idée maîtresse de créativité diffuse (plutôt que centralisée) pour traiter les aspects de durabilité sociale et environnementale tant débattus dans les arènes internationales?

Un certain nombre de stratégies opérationnelles ont été proposées pour contribuer à renforcer les liens et la cohérence entre les différents secteurs de l'action et des politiques. Beaucoup d'entre elles relèvent de l'idée des partenariats public-privé et, plus généralement, s'appuient sur le rôle du secteur privé comme un des moteurs de l'innovation. Dans la pratique cependant, la mise en place de partenariats s'est révélée difficile. Même lorsque de nouvelles alliances ont été forgées, leur gouvernance pour orienter l'innovation dans le sens d'un programme de développement à la fois social et durable constitue le véritable point d'achoppement.

\section{"Les sources alternatives de perturbation : l'esprit d'entreprise et le développement}

Le paradoxe qui saute aux yeux est que, tandis que nous luttions pour construire des systèmes d'innovation agricoles pour les pauvres sous des intitulés grandioses, une multitude d'innovation à la fois socialement pertinentes et durables se développaient tout autour de nous depuis des années. Le BRAC, Amul, Real IPM, FIPS et Gram Mooligai sont des exemples de la diversité croissante de ces initiatives, certaines anciennes, d'autres plus récentes, allant du mouvement Chipko des femmes pour la protection de l'environnement en Inde, au système de riziculture intensive (une approche innovante et sobre en intrants de la riziculture), aux microcrédits de Mohammed Yunnus au Bangladesh, à la philosophie de la technologie intermédiaire de Schumacher, à la Campaign for Real Ale («campagne pour la bière véritable ») au Royaume-Uni, ainsi qu'à beaucoup d'autres ${ }^{2}$.

À des degrés différents, toutes ces initiatives ont perturbé les modes de production et d'innovation en vigueur. Pourtant, pour des raisons de perception et d'analyse, de nombreux projets de ce genre sont mis en œuvre sans que les marchés et les politiques s'en aperçoivent, dans l'ombre de leur radar. Bien entendu, nombre d'entre eux se soldent simplement par des échecs, et très peu parviennent à percer au niveau national et international, en dépit des opportunités qu'ils représentent économiquement et socialement. Nous sommes nous trompés sur ce qui fait réellement avancer l'innovation, au point de ne pas avoir su voir de remarquables bouillons de créativité ?

Il apparaît de manière flagrante que de nombreux défis et opportunités existent en matière de développement agricole et rural auxquels les innovations conventionnelles axées sur les marchés ne donneront pas suite mais qui, pour des raisons sociales et environnementales, n'en sont pas moins importants.

Il peut être intéressant ici de revenir sur un des premiers articles traitant de l'innovation, rédigé dans les années 1930 par l'autrichien Joseph Schumpeter, expert

2. voir http://aylluinitiative.org/ pour une tentative de cartographie mondiale de ces initiatives. 
en économie et en science politique. Il désigne alors l'esprit d'entreprise comme la force créatrice centrale, évoquant un processus de perturbations successives, où l'on fait quelque chose de différent dans l'espoir d'un profit, une destruction créatrice de l'ancien pour introduire du neuf $(1934,1950)$.

Selon Schumpeter, les entrepreneurs sont les forces motrices nécessaires au progrès économique, sans lesquelles les économies s'immobilisent et dépérissent. Ils sont des entités qui repèrent une opportunité commerciale et montent une opération afin d'y donner suite. Le bon esprit d'entreprise démarre une réaction en chaîne, soutient Schumpeter, poussant d'autres entrepreneurs à propager l'innovation jusqu'au point de « destruction créatrice » : « un état dans lequel la nouvelle opération et toutes celles qui y sont liées rendent concrètement obsolètes les produits, les services et les modèles commerciaux pré-existants ». Ainsi Schumpeter imaginait-il l'entrepreneur œuvrant au sein d'un système plus large, suscitant le changement par l'exemple et prospérant sur ce changement comme s'il s'agissait d'un combustible pour poursuivre dans l'action.

Les entrepreneurs - sociaux, environnementaux ou liés au marché - guettent les opportunités et, fréquemment, complètent ou suppléent l'action défaillante du secteur public ou d'autres acteurs (Mair, 2008). Tandis que le profit économique reste une dimension souvent prise en considération, il ne faut pas pour autant oublier les divers objectifs de développement poursuivis par ailleurs. Dans bien des cas, l'esprit d'entreprise ne vise pas uniquement l'accumulation de gains financiers : il s'agit plutôt de générer de la valeur ajoutée - sociale, commerciale ou environnementale dans un contexte sociétal donné (Dees, 1998).

Outre la prise de risque, à quoi fait appel l'esprit d'entreprise ? Faire preuve d'esprit d'entreprise, c'est prendre la responsabilité de mobiliser des idées, des ressources, des personnes, des processus, des institutions et des politiques pour produire quelque chose de nouveau. Bien entendu, il s'agit là précisément du type de processus que la pensée sur les systèmes d'innovation défendait, bien qu'elle se soit parfois perdue à vouloir trop orchestrer.

\section{"Que trouve-t-on dans les zones d'ombre du radar?}

Ayant reconnu que c'est l'esprit d'entreprise dans son sens général, plutôt que le secteur privé ou les entreprises, qui nous intéresse, nous pouvons dorénavant distinguer différentes formes d'entrepreneuriat et d'innovation, ainsi que le potentiel de synergies entre eux : entrepreneuriat de marché (pour le profit), entrepreneuriat social (pour le changement social), entrepreneuriat environnemental (pour la protection de l'environnement).

Ainsi que la littérature consacrée à l'entrepreneuriat social tient à le faire remarquer (Mair, 2008 ; Gries et Naudé, 2011), ces distinctions masquent le fait que les entreprises sociales ont souvent en parallèle des visées commerciales et environnementales. Ces entreprises comptent également parmi elles des chercheurs jouant le rôle d'entrepreneurs sociaux parce qu'ils y ont vu un moyen de valoriser leurs travaux scientifiques. Le plus intéressant, cependant, est qu'il existe une catégorie 
grandissante d'entrepreneurs qui lancent des initiatives pionnières qui se situent explicitement à la croisée des préoccupations sociales, environnementales et de marché (le point $x$ dans la figure 3.1). Toutes les initiatives citées dans l'introduction appartiennent à cette catégorie.

Ce type d'entreprenariat hybride, actuellement dans les zones d'ombre du radar, ne doit pas être confondu avec la responsabilité sociale des grandes entreprises. Il comprend des organisations œuvrant explicitement à la fois pour leur profit et pour changer les choses. Le point essentiel dont il convient de tenir compte ici est que ces entrepreneurs ne sont pas nécessairement des adeptes du profit maximal, mais des personnes et des groupes qui se satisfont pleinement de vivre en opérant un compromis entre faire des profits et contribuer à un monde meilleur. Ils comptent parmi eux des présumés activistes sociaux comme des innovateurs-perturbateurs. Ils présentent souvent des tendances iconoclastes, défiant ce qu'ils voient autour d'eux comme des anciennes méthodes dépassées et infondées.

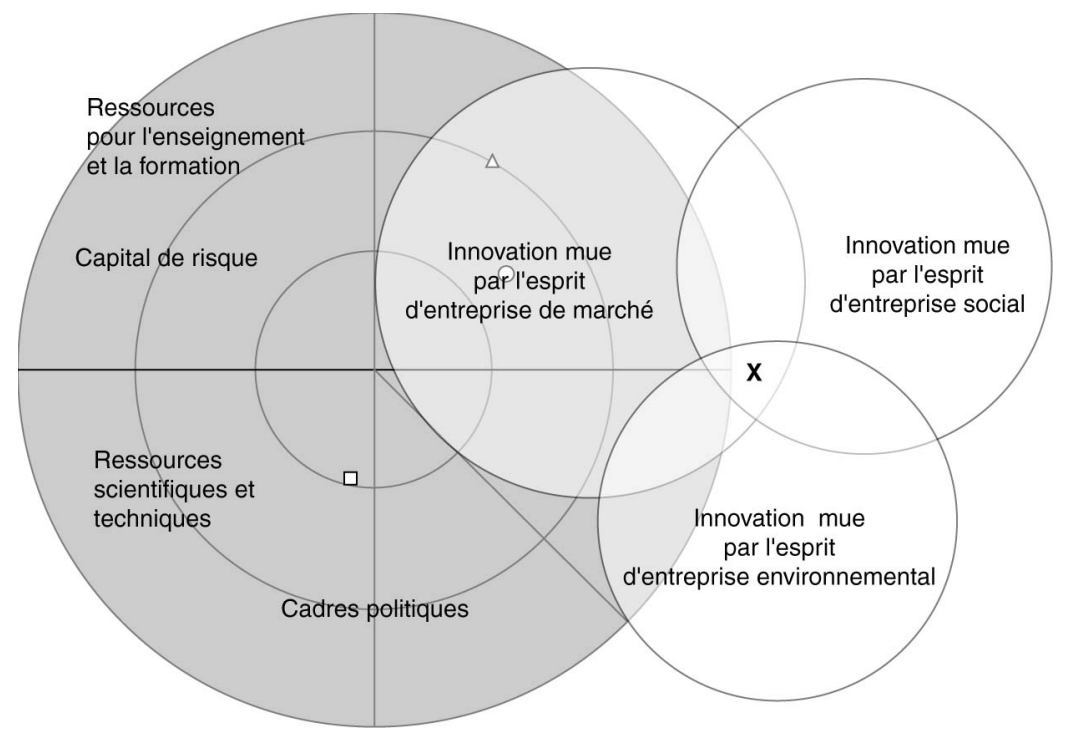

Figure 3.1. Que trouve-t-on dans les zones d'ombre?

Le rayonnement grandissant de ces entrepreneurs dans la zone d'ombre du radar est dû :

- aux limites dans la capacité des grandes entreprises conventionnelles à innover pour gagner les grands marchés des populations les plus pauvres ;

- aux limites des modes d'innovation à l'œuvre dans les zones de production écologiquement fragiles ;

- au déplacement du centre de gravité de l'innovation à l'échelle de la planète, depuis le monde des grandes entreprises de l'hémisphère Nord vers de nouvelles zones de dynamisme économique et social dans les pays du Sud.

À l'Open University britannique, Kaplinsky et al. (2010) soutiennent que ces nouveaux modes d'innovation par perturbations vont jouer un rôle de premier plan en Inde et en Chine, avec des répercussions possibles à l'échelle mondiale. 
Il n'existe pas d'entrepreneur type, mais nous commençons maintenant à nous faire une idée relativement précise des attributs qui le caractérisent :

- auto-organisateur, mû par les opportunités : les individus et les groupes voient les opportunités comme des chances à saisir. Il peut s'agir d'une opportunité de marché (pour le commerce équitable, par exemple, mais également en voyant les populations les plus pauvres comme d'énormes débouchés de marché), une opportunité technique (telles que des avancées technologiques dans le domaine des énergies renouvelables ou des bio-pesticides) ou une opportunité suite à une réorganisation des activités dans un but social (telle que le microcrédit) ;

- réclamant des changements de modèle : presque par définition, les approches de la zone d'ombre réclament des changements dans la manière dont les politiques et les institutions organisent le monde. La société Real IPM a dû lutter pour obtenir les modifications réglementaires permettant l'autorisation et la mise sur le marché d'agents de bio-lutte au titre de «pesticides ». Les mouvements en faveur des produits biologiques partout dans le monde ont eu le succès qu'on leur connaît parce qu'ils ont pu faire amender les réglementations s'appliquant à l'étiquetage des aliments. Le mouvement qui promeut le système de riziculture intensive continue à défier la vision scientifique de la production rizicole ;

- souvent enlisé : tout comme dans le monde des affaires, quantité d'initiatives sociales dans la zone d'ombre échouent. Beaucoup ne sont simplement pas très bonnes. Alors que d'autres ne peuvent plus avancer. Il arrive que les réseaux ne soient pas suffisamment larges pour fournir le soutien technique dont ces entreprises ont besoin pour s'adapter aux évolutions du marché ou de la réglementation, comme

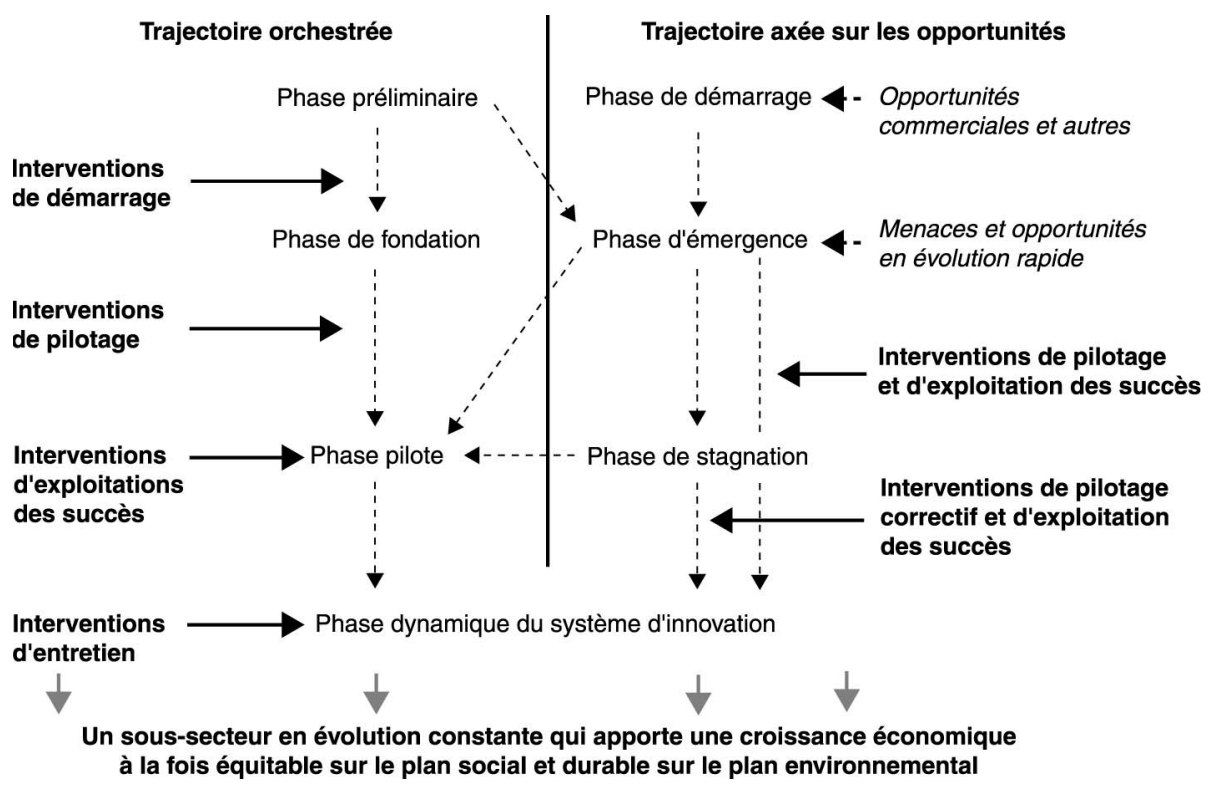

Figure 3.2. Des trajectoires d'innovation contrastées (d'après Banque mondiale, 2006). 
dans le cas de certains projets de commerce équitable. Le secteur des biocarburants au Sénégal s'est enlisé parce qu'aucune coalition d'entreprises et d'ONG travaillant dans ce secteur n'était présente pour faire pression de manière concertée en faveur d'une politique plus propice. Le secteur du biogaz en Suisse a réussi, quant à lui, grâce à une telle coalition. Le mouvement pour les technologies intermédiaires a fini par s'essouffler car il n'arrivait pas à dépasser le travail d'adaptation technique pour faire face aux nouvelles réglementations politiques. Les modes de financement disponibles sont presque toujours à trop court terme pour permettre l'incubation de projets susceptibles d'apporter un changement à la fois social et institutionnel - qui peuvent nécessiter plus de dix ans pour aboutir.

\section{W Conclusions et conséquences en matière de politiques}

La voie à suivre pour aller de l'avant est de consacrer beaucoup plus d'attention politique au soutien des entrepreneurs sociaux de la zone d'ombre du radar. Nous devrions arrêter d'essayer de construire de nouveaux systèmes d'innovation socialement pertinents et mettre plutôt en application les principes des systèmes d'innovation pour soutenir les modes d'innovation hybrides qui inaugurent des modèles d'entreprise répondant aux objectifs de développement les plus cruciaux dans la plupart des pays du Sud, mais également dans le Nord.

Des opportunités existent également ici pour les entreprises et le secteur privé. Beaucoup de ces entrepreneurs de la zone d'ombre sont en train de poser les premiers jalons de modèles d'entreprise qui trouvent des moyens d'accéder à des grands marchés de populations pauvres - une tâche que, pour des raison de taille, de structure et d'optique, bien des grandes entreprises n'ont pas su mener à bien. Il est vraisemblable que ces modèles exerceront une forte attraction sur les entreprises qui sont arrivées aux limites de leur part de marché dans les marchés existants.

Ceci implique quelques modifications générales des politiques pour soutenir l'innovation :

- élargir le mainstream vers une diversité de courants : offrir un soutien à des entrepreneurs et des modes d'entrepreneuriat très divers, occupant une multitude de niches et s'attaquant par divers moyens à différents objectifs, de marché, sociaux ou de durabilité ;

- passer d'une logique de modèles reproductibles à la création d'une mosaïque de succès de niche : au lieu de rechercher des modèles pilotes susceptibles d'être copiés et transposés en vue de nouvelles approches de type « industriel », l'accent doit être mis sur l'identification d'une grande diversité d'entreprises prometteuses et sur l'accompagnement de leur développement ;

- de l'orchestration à la facilitation : l'innovation étant en grande partie autoorganisatrice (du moins au tout début) et mue par l'esprit d'entreprise, les politiques doivent se donner un rôle de soutien ; les activités telles que la recherche doivent être organisées comme des ressources facilement exploitables ; l'incubation et la création d'espaces pour les premiers stades des activités entrepreneuriales deviendront beaucoup plus importantes. 


\section{" Conséquences pratiques}

L'idée à retenir, pour ce qui est des opérations de planification, est que l'accent ne doit plus être mis sur les tentatives de construction de nouveaux systèmes, mais plutôt sur le soutien des modes de créativité en émergence. Ce soutien doit être étendu à l'instauration des conditions permettant en premier lieu à l'innovation des zones d'ombre d'émerger. La figure 3.2 présente en face à face, d'une part, la manière dont les interventions des politiques peuvent être organisées en trajectoires d'innovation orchestrées et, d'autre part, la trajectoire axée sur les opportunités que saisissent les entrepreneurs. Les grandes catégories d'intervention et de soutien proposées ci-dessous seront cruciales :

- la détection : un rôle de premier plan pour la recherche sera de tenter de détecter les nouveaux processus d'innovation prometteurs, qui étaient jusque là dans les zones d'ombre, ayant une forte pertinence sur le plan social et de la durabilité. Une part importante du travail consistera à trier et à éliminer les initiatives dont la probabilité d'échec est élevée ;

- le financement : de nouvelles formes de financement devront être mises à disposition - non seulement des fonds d'encouragement (challenge funds), mais également de nouveaux types de capitaux-risque à objectif social. Il est également important de reconnaître que les retours sur investissement peuvent, avec ce type d'initiatives, ne se manifester qu'après un temps relativement long, du fait de la durée plus importante de la période d'incubation et de la nécessité de faire évoluer les politiques ;

- les services de soutien adaptatifs : la phase initiale de ces initiatives est certes auto-organisatrice, mais correspond par ailleurs à la période pendant laquelle un certain soutien est nécessaire. Des services adaptatifs doivent permettre de faciliter le changement dans les domaines techniques, institutionnels et politiques et également fournir un accompagnement professionnel, une formation lors de l'incubation, des possibilités de réseautage et de financement, ainsi qu'un soutien aux activités de recherche ;

- la réduction des risques : il est important de prévoir des mécanismes - tels que des incitations fiscales, des subventions et de nouveaux mécanismes de financement - pour réduire les risques auxquels sont exposées les nouvelles activités entrepreneuriales.

Le paradoxe suprême est que tandis que nous rêvions à la forme des systèmes d'innovation agricoles du futur - si seulement nous avions pu les voir - ils étaient à nos côtés tout du long. Le défi consiste aujourd'hui à trouver les moyens de structurer un soutien pour les dynamiques d'innovation en évolution constante pour lesquelles les catégories organisationnelles du $\mathrm{xx}^{\mathrm{e}}$ siècle sont rapidement en passe de devenir obsolètes.

\section{" Références bibliographiques}

Banque mondiale, 2006. Enhancing agricultural innovation: how to go beyond the strengthening of research systems, Economic Sector Work report, La Banque Mondiale, Washington DC, pp. 149.

Biggs S.D., 1990. A multiple source of innovation model of agricultural research and technology promotion. World Development, 18 (11), 1481-1499. 
Biggs S.D., Clay E.J., 1981. Sources of innovations in Agricultural Technology. World Development, 9, 321-336.

Dees J.G., 2001. The Meaning of Social Entrepreneurship, Center for the Advancement of Social Entrepreneurship (CASE), May 30.

Engel P.G.H., 1995. Facilitating innovation: an action-oriented approach and participatory methodology to improve innovative social practice in agriculture, Wageningen University, Wageningen, The Netherlands.

Freeman C., 1995. The 'National System of Innovation' in Historical Perspective. Cambridge Journal of Economics, 19 (1), 5-24.

Gries T., Naudé W.A., 2011. Entrepreneurship and human development: A capability approach. Journal of Public Economics, 95 (3), 216-224.

Hall A., 2006. Public-private sector partnerships in a system of agricultural innovation: concepts and challenges. International Journal of Technology Management and Sustainable Development, $5(1)$.

Hall A.J., 2005. Capacity development for agricultural biotechnology in developing countries: an innovation systems view of what it is and how to develop it. Journal of International Development, 19(5), 611-630.

Hall A.J., 2009. Challenges to strengthening agricultural innovation systems: Where do we go from here? In : Farmer first revisited: Farmer-led innovation for agricultural research and development (Scoones I., Chambers R., Thompsons J., eds.), Practical Action, United Kingdom, 30-38.

Hall A.J., Rasheed Sulaiman V., Clark N.G., Sivamohan M.V.K., Yoganand B., 2002. Public-private sector interaction in the Indian agricultural research system: An innovation systems perspective on institutional reform, In : Agricultural research policy in an era of privatization: Experiences from the developing world (Byerlee D., Echeverria R.G., eds), CABI.

Hall A., Sivamohan M.V.K., Clark N., Taylor S., Bockett G., 1998. Institutional developments in Indian agricultural $\mathrm{R}$ and $\mathrm{D}$ systems: Emerging patterns of public and private sector activities. Science, Technology and Development, 16 (3), 51-76.

Juma C., 2010. The New Harvest: Agricultural Innovation in Africa, Oxford University Press.

Kaplinsky R., Chataway J., Clark N., Hanlin R., Kale D., Muraguri L., Papaioannou T., Robbins P., Wamae W., 2010. Below the radar: what does innovation in emerging economies have to offer other low income economies? International Journal of Technology Management and Sustainable Development, 8 (3).

Leeuwis C., Pyburn R., 2002. Wheelbarrows full of frogs: social learning in rural resource management, Koninklijke Van Gorcum, Assen.

Mair J., 2008. Social entrepreneurship: Taking stock and looking ahead. Paper presented at the 2008 World Entrepreneurship Forum.

Roling B., 1992. The emergence of knowledge systems thinking: A changing perception of relationships among innovation, knowledge process and configuration. Knowledge and Policy, 5 (1), 42-64.

Schumpeter J.A., 1934. The theory of economic development, Harvard University, Cambridge MA. Schumpeter J.A., 1950. Capitalism, socialism and democracy, Harper \& Row, New York. 



$$
\begin{gathered}
\text { Apprtie } 2 \\
\begin{array}{c}
\text { aujourdre à innover } \\
\text { aui }
\end{array}
\end{gathered}
$$





\title{
Chapitre 4 \\ Innover dans les systèmes de culture et de production
}

\author{
Jean-Marc MeYnARD
}

Les systèmes agricoles intensifs, fortement utilisateurs d'intrants chimiques (engrais, pesticides, produits vétérinaires), et largement mécanisés, dominent aujourd'hui l'agriculture des pays développés et s'affirment aussi dans de nombreux pays du Sud. Ils sont généralement plus productifs à l'hectare que les systèmes traditionnels; ils sont également le plus souvent économes en main d'œuvre, et ont permis un accroissement sans précédent de la productivité du travail. On s'interroge cependant de plus en plus sur la durabilité de ces systèmes intensifs en intrants : ils sont gourmands en énergie fossile, producteurs de gaz à effet de serre, et globalement défavorables à la biodiversité ; ils polluent les eaux par des nitrates, des composés phosphoriques et des pesticides, etc. S'ils ont contribué à faire reculer les famines et à faire baisser le coût de l'alimentation des urbains, ils ont aussi participé à concentrer la production entre les mains de ceux qui avaient les moyens d'investir dans les intrants et dans la mécanisation, et contribué à accroître la vulnérabilité des paysans qui n'avaient pas accès à ces moyens (de Schutter, 2010).

La question est maintenant posée : ces systèmes intensifs en intrants doivent-ils se généraliser, en corrigeant leurs plus graves défauts, ou faut-il leur substituer d'autres formes d'agriculture? Quels systèmes de production peut-on imaginer pour assurer aux populations rurales et urbaines la sécurité alimentaire et aux paysans un revenu régulier et suffisant, tout en économisant les ressources naturelles, et en favorisant l'expression des services écosystémiques et la cohésion sociale au niveau des territoires (FNH, 2009)?

Dans ce chapitre, nous analysons les logiques agronomiques des systèmes actuels, et leurs déterminants économiques et sociaux, à partir d'exemples divers, en France et au Sud, pour apprécier leurs marges d'évolution, et le pas à franchir pour les rendre durables. Nous montrons qu'il faudra changer de logique agronomique, réinventer des systèmes agricoles en mobilisant des démarches d'ingénierie agro-écologique. Nous en tirons quelques propositions, concernant les orientations de la recherche \& développement agricole et de l'action publique, visant à favoriser les nécessaires évolutions. 


\section{W Logiques agronomique, économique et sociale des systèmes agricoles intensifs en intrants : un exemple en France}

L'intensification par les intrants s'inscrit dans des logiques globales, qui doivent être analysées simultanément aux plans agronomique, économique et social, et en prenant en compte non seulement ce qui se passe au niveau des exploitations agricoles, mais également des territoires et des filières. L'interconnexion de ces différents plans sera illustrée sur le cas des systèmes céréaliers du bassin de la Seine, au nord de la France.

\section{La spécialisation des territoires et des systèmes de production}

Depuis les années 1960, où ils dominaient l'agriculture du bassin de la Seine, les systèmes de polyculture-élevage ont partout reflué. Les régions du centre du Bassin, où les terres sont les plus fertiles, se sont spécialisées en céréaliculture, et l'élevage y a presque disparu. Dans les régions périphériques, l'élevage bovin a été intensifié par le développement du maïs ensilage et l'importation de tourteaux de soja. La figure 4.1, tirée de Schott et al. (2010), illustre les conséquences de cette évolution au niveau de l'occupation des sols : les cartes représentent l'assolement du bassin de la Seine (au nord-ouest, la côte normande, à l'est, la Bourgogne), soit environ $100000 \mathrm{~km}^{2}$, lors des recensements effectués par le ministère de l'Agriculture entre les années 1970 et 2000. On observe un accroissement des surfaces en céréales, et principalement en blé, particulièrement marqué dans la zone centrale du bassin (Îlede-France, Beauce, Brie, Picardie) : le blé occupe en 2000 dans certaines régions plus de $50 \%$ de la surface agricole. La régression de l'élevage de ruminants dans cette zone centrale se traduit par la forte diminution des surfaces en prairies naturelles (non illustré). En 2000, elles n'occupaient des superficies significatives que dans les zones périphériques, telles que la Normandie à l'ouest ou la Thiérache au nord. La spécialisation vers l'élevage intensif dans ces régions est marquée par le développement spectaculaire des surfaces en maïs ensilage (figure 4.1). Cette spécialisation des exploitations et des régions pose de nombreux problèmes écologiques : faible recyclage des éléments minéraux $(\mathrm{N}, \mathrm{P}, \mathrm{K}, \ldots)$ dans les exploitations agricoles, qui conduit à un gaspillage de ressources non renouvelables, et à des pollutions de l'eau (nitrate, phosphore) et de l'air (ammoniac, oxyde nitreux); perte de biodiversité liée au remplacement des prairies par des cultures annuelles ; réduction de la diversité des mosaïques d'habitats défavorable à la biodiversité...

Le cas de la luzerne, illustré par la figure 4.2 (Schott et al., 2010), est emblématique de ce processus de spécialisation régionale. Dans les années 1970, la luzerne était présente sur tout le bassin de la Seine, en général consommée par le bétail dans les fermes où elle était cultivée. Son déclin a suivi celui des systèmes de polyculture élevage, et entre 1980 et 2000, la luzerne s'est concentrée en Champagne crayeuse, dans une zone favorable à une production élevée (sols profonds à très forte réserve en eau), pour alimenter la filière «déshydratation »: la luzerne est déshydratée grâce à l'énergie fossile, pour pouvoir être facilement conservée, transportée, et 
A
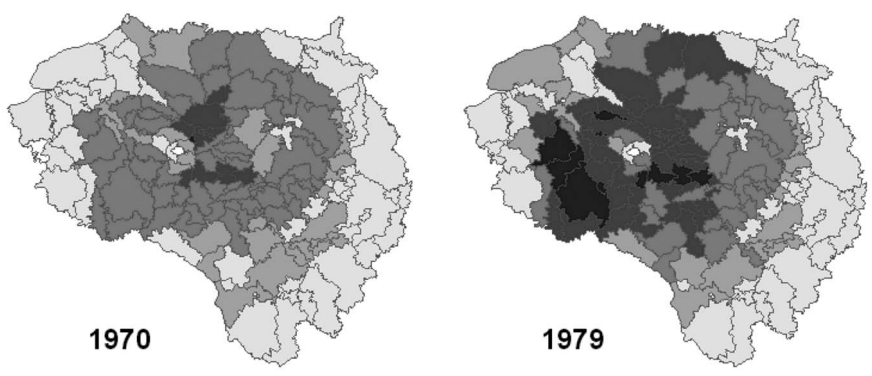

Surfaces en blé tendre en $\%$ de la SAU
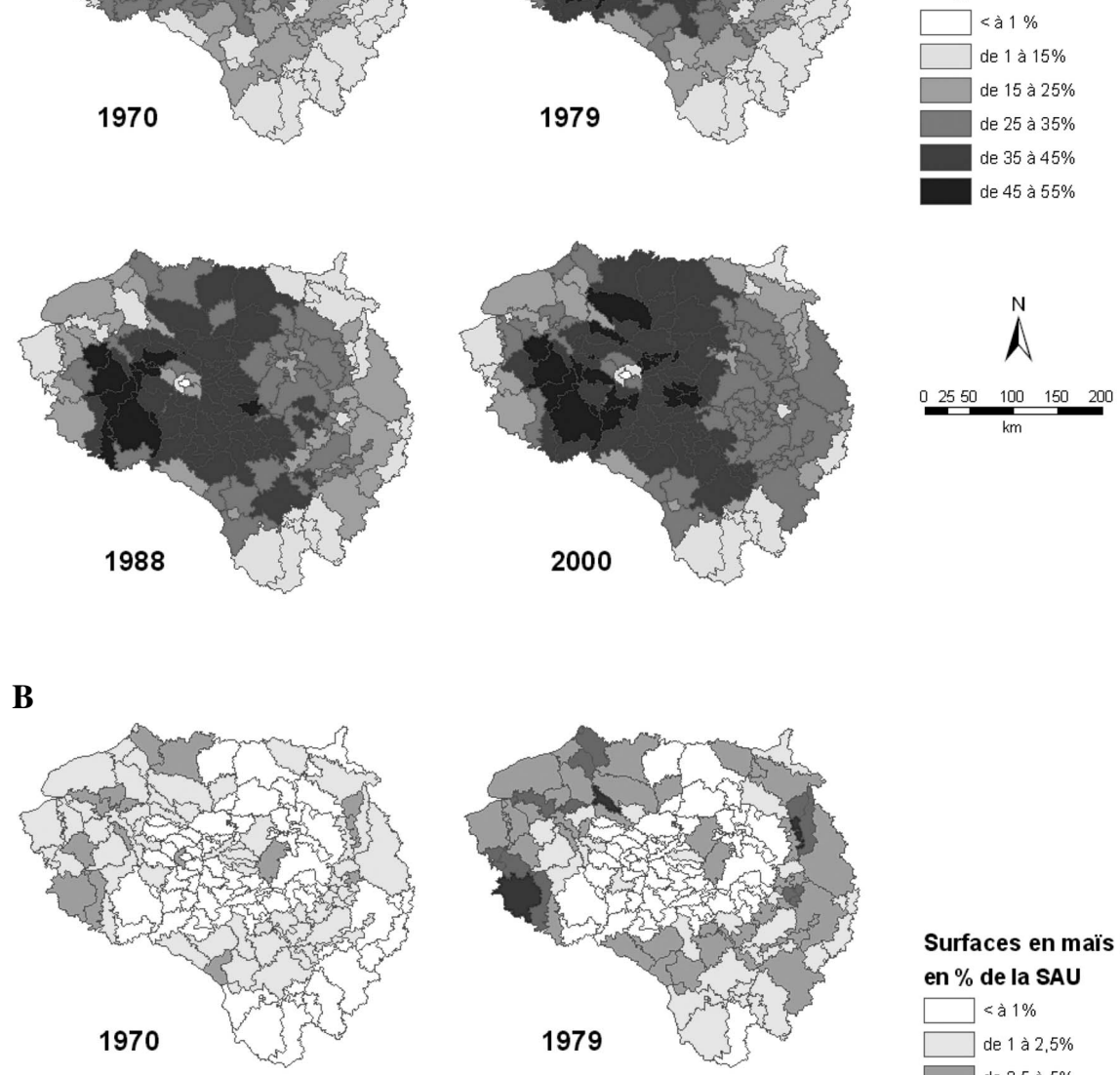

\section{Surfaces en maiis fourrage} en $\%$ de la SAU
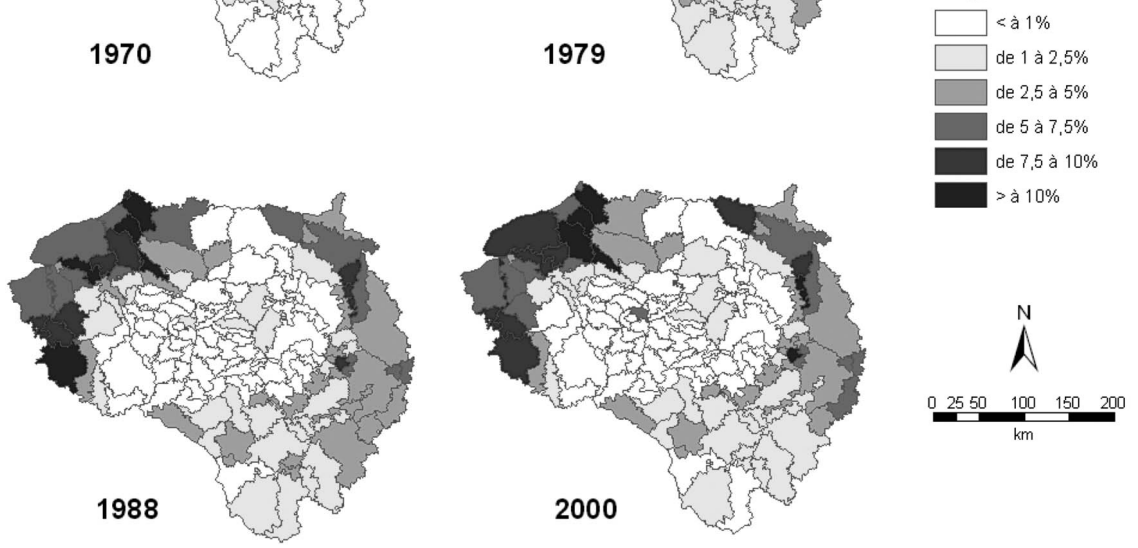

Figure 4.1. La spécialisation des territoires dans le bassin de la Seine : au centre la céréaliculture $(\mathrm{A})$, à la périphérie l'élevage (B, marqué par les surfaces en maïs ensilage) ; d'après Schott et al., 2010. 
incorporée dans des aliments du bétail vendus aux éleveurs des régions spécialisées en élevage. Les années 2000 marquent un déclin de cette filière énergivore, lié à la hausse du prix de l'énergie, à la baisse du soutien européen et à la concurrence du tourteau de soja venu du continent américain.
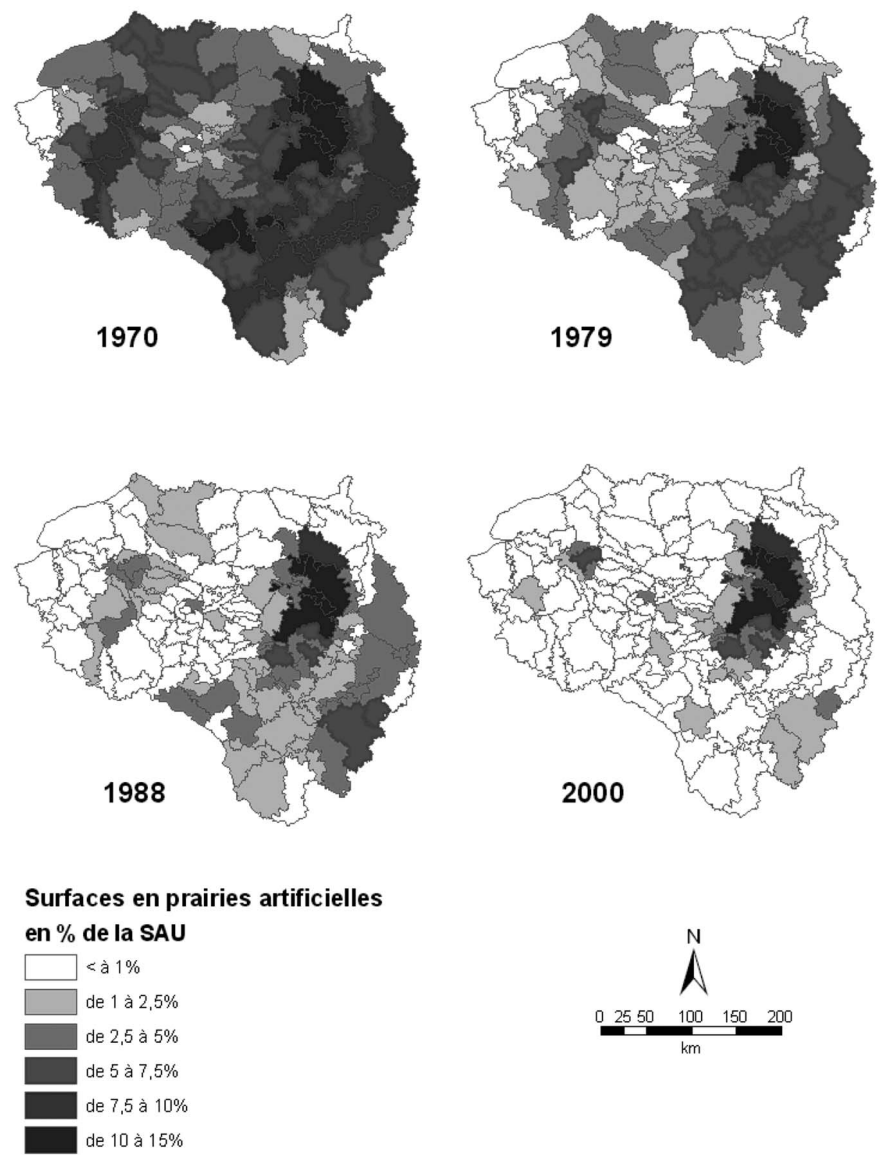

Figure 4.2. La diminution des surfaces en luzerne dans le bassin de la Seine, emblématique d'une agriculture peu économe (d'après Schott et al., 2010).

Cette spécialisation des territoires est structurée par les implantations agroindustrielles qui assurent les débouchés aux produits : l'industrie laitière, qui collecte l'essentiel de la production de lait s'est concentrée dans les zones d'élevage ; inversement, les régions centrales se sont organisées pour collecter, et parfois transformer (sucreries, féculeries par exemple) les produits des grandes cultures. La spécialisation s'accompagne d'un accroissement de la technicité des modes de production, et les agriculteurs spécialisés, comme leurs conseillers, ne ressourcent leurs compétences que dans leurs domaines de spécialisation. Se re-diversifier devient alors d'autant plus difficile pour un agriculteur qu'il lui faut trouver non seulement des débouchés qui n'existent plus localement, mais aussi acquérir sans appui local les savoirs et les références techniques sur les nouvelles productions. 


\section{La réduction du nombre d'espèces cultivées}

Cette spécialisation régionale s'accompagne d'une réduction du nombre d'espèces cultivées, et d'un raccourcissement des rotations. Ainsi, dans les zones de grande culture du bassin de la Seine, les surfaces en blé et en colza ont beaucoup augmenté entre les années 1980 et 2000. À l'opposé, les surfaces en pois protéagineux, en tournesol ou en maïs ont diminué. Les monocultures de blé ou les rotations courtes, telles que colza/blé/blé ou colza/blé/orge, ont augmenté en fréquence (Schott et al., 2010). Toute la filière est impliquée dans cette simplification des assolements : l'exemple de la régression du pois protéagineux (surfaces divisées par 7 en 15 ans en France entre 1994 et 2009) en est l'illustration. Le développement des surfaces en pois protéagineux dans les années 1980 est dû à un soutien résolu des pouvoirs publics (prix élevé garanti, soutien à la sélection variétale et à la mise au point de références techniques), lié à une volonté de réduire la dépendance de l'Union européenne en ressources protéiques pour l'alimentation animale. Le déclin des surfaces s'est amorcé suite à une réduction du soutien des pouvoirs publics, mais aussi en relation avec le développement d'une nouvelle maladie tellurique, Aphanomyces euteiches. Dans la période d'expansion maximale des surfaces en pois, au tournant des années 1980 et 1990, certains agriculteurs ont en effet cultivé un peu trop souvent du pois sur les mêmes parcelles, ce qui a favorisé l'expansion de la maladie. La régression des surfaces a décrédibilisé le pois vis-à-vis des fabricants d'aliments du bétail, qui ne disposaient plus d'un approvisionnement régulier et garanti : le tourteau de soja a retrouvé sa place de source essentielle de protéines dans la composition des aliments du bétail. Les prix du pois en ont pâti, ce qui a accéléré la baisse des surfaces. Les semenciers privés se sont alors interrogés sur l'avenir du pois et ont réduit leur investissement dans la sélection de nouvelles variétés. Le différentiel de productivité avec le blé ou le colza, sur lesquels l'effort de sélection ne se relâche pas, ne se réduira donc pas, ce qui serait nécessaire pour accroître l'intérêt économique au pois protéagineux. C'est bien l'imbrication des stratégies des différents acteurs de la filière qui a abouti au déclin du pois, et qui compliquera singulièrement un retour du pois dans les assolements aujourd'hui souhaité par les pouvoirs publics.

\section{Le rôle clef des pesticides}

Le raccourcissement des rotations augmente les problèmes de parasitisme tellurique et rend difficile la maîtrise des populations d'adventices. L'homogénéisation des assolements accroît les risques de développement des populations de parasites à dissémination aérienne (insectes, maladies cryptogamiques foliaires). Le processus de spécialisation que nous venons de décrire ne serait donc pas possible sans les pesticides. À titre d'illustration, on peut observer que dans le bassin de la Seine, les régions où il y a le plus de colza sont aussi celles dans lesquelles chaque hectare de colza reçoit le plus de traitements pesticides (Schott et al., 2010). De fait, les pesticides sont devenus la clé de voûte des systèmes de culture intensifs actuels, et configurent non seulement les rotations, mais aussi les dates de semis ou les choix variétaux. L'exemple de la culture du blé (Meynard et Girardin, 1991; Lamine et al., 2010) est à cet égard significatif. Pour maximiser la productivité, le blé est semé précocement et à densité forte, il est alimenté régulièrement en engrais azoté, les 
variétés sont choisies en fonction de leur productivité (et non de leur résistance aux maladies). Tous ces choix sont favorables à des niveaux élevés de production, mais ils accroissent aussi les risques d'insectes parasites, de maladies cryptogamiques et d'adventices. Face à ces risques, une couverture serrée de la culture par des traitements phytosanitaires devient impérative. Pour faciliter des interventions rapides, les agriculteurs ont privilégié l'achat de matériels puissants et de grande largeur, et cherché à regrouper et agrandir leurs parcelles. C'est ainsi que dominent dans les paysages de grandes cultures, des systèmes intensifs, pratiqués sur de grandes parcelles génétiquement homogènes, et fortement utilisateurs de pesticides.

Ce rôle clé joué par les pesticides dans les régions de grande culture est renforcé par le système de conseil et les priorités de la sélection. Du fait du rôle clef des pesticides dans la logique des systèmes de culture, les entreprises qui commercialisent ces intrants sont devenues la principale source de conseil aux agriculteurs. Pour lutter contre les bio-agresseurs, ce conseil privilégie le plus souvent les solutions chimiques, simples et d'efficacité spectaculaire (un problème, une solution) plutôt que les méthodes agronomiques préventives, plus complexes à mettre en œuvre et d'efficacité moins directe (Butault et al., 2010). Les résistances variétales sont le plus souvent considérées comme des compléments aux pesticides, et non comme des moyens de lutte privilégiés. De ce fait, le marché des variétés multi-résistantes, qui autorisent des réductions de fongicides significatives, reste limité, ce qui n'incite pas les sélectionneurs à privilégier ce créneau. Étant donné le rôle secondaire des résistances variétales, il n'y a pas de coordination des choix variétaux en vue d'une gestion de la durabilité des résistances. Le contournement de résistances variétales est de ce fait régulièrement observé, et tend à décrédibiliser cette solution.

\section{Un système verrouillé}

Les systèmes de production de la région étudiée apparaissent ainsi comme totalement cohérents avec l'organisation de filières amont et aval, ainsi qu'avec les systèmes de conseil technique. La stratégie de chaque acteur renforce la stratégie des autres. Personne n'a vraiment intérêt à changer de stratégie, tant que les autres n'en changent pas. On est dans un cas typique de «verrouillage technologique ${ }^{1}$ » autour de ces systèmes agricoles spécialisés et intensifs en intrants. Des effets de verrouillage autour de l'utilisation des pesticides ont déjà été décrits dans d'autres pays - Cowan \& Gunby (1996) aux USA, Vanloqueren et Baret (2008) en Belgique et en France par Lamine et al. (2010). La forte cohérence du système sociotechnique qui produit ce verrouillage est le résultat de la remarquable réponse du monde agricole à l'injonction d'accroissement de la production de céréales, et à l'augmentation de la compétitivité internationale de l'agriculture. Cette cohérence se heurte clairement à une prise en compte des nouvelles injonctions relatives à l'environnement. Comme le soulignent les travaux des économistes sur la suppression des quotas laitiers (Daniel et al., 2008) ou sur la diversification des cultures (Farès et al.,

1. Le concept de verrouillage technologique traduit une situation dans laquelle une technologie A peut être adoptée de façon durable voire irréversible au détriment d'une technologie $\mathrm{B}$, et ce même si la technologie B apparaît, ex-post, comme étant la plus efficace (Labarthe, 2010). 
2012), les mécanismes économiques au niveau des filières tendent à renforcer ce verrouillage. Selon le processus de dépendance du chemin (path dependancy) décrit par les économistes de l'innovation (David, 1985 ; Dosi, 1988), certaines innovations, cohérentes avec ce verrouillage (rotations encore plus courtes, outil d'aide à la décision permettant de raisonner les apports d'intrants) ont beaucoup plus de chance d'être adoptées que les autres (Labarthe, 2010). L'évolution possible pour les systèmes est donc strictement limitée : ouvrir le champ des possibles en termes d'innovation suppose de « déverrouiller ».

\section{"Quelles marges de manœuvre pour faire évoluer les systèmes intensifs en intrants ?}

Des systèmes sociotechniques analogues à celui décrit dans le Bassin parisien en France prédominent largement dans les pays développés. Mais ils s'affirment aussi dans des régions agricoles des pays du Sud. Pour saisir à quel point il s'agit d'une évolution globale des systèmes agricoles, qui s'ancre dans des dynamiques décennales et repose sur des acteurs internationaux et les règles du commerce mondial, nous prendrons trois autres exemples emblématiques de cette évolution, dans des contextes écologiques et socio-économiques contrastés : la sojisacion en Pampa argentine, la production de coton en Thailande et la production bananière aux Antilles.

\section{La sojisation de la Pampa argentine (d'après Grosso, 2011)}

Depuis le milieu des années 1990, on assiste dans la Pampa argentine à un fort développement des grandes cultures, et particulièrement du soja, aux dépens des prairies et de l'élevage : la surface en soja dans le pays a ainsi été multipliée par 4 (de 500000 ha à 2 millions d'ha) entre le début des années 1990 et la fin des années 2000. Les variétés de soja cultivées sont, en quasi-totalité, tolérantes aux herbicides (glyphosate en premier lieu). Ce développement du soja est directement lié au fait que sa marge brute est supérieure à celle des autres cultures. Aujourd'hui, environ $60 \%$ des surfaces cultivées sont ensemencées en soja, et de plus en plus de rotations comprennent un retour du soja sur lui-même. Le développement du soja s'est accompagné d'une suppression presque généralisée du labour, ce qui permet une réduction du temps de travail et des coûts de mécanisation. Il existe clairement une synergie entre les deux innovations, semis direct et variétés tolérantes aux herbicides : la suppression du labour suppose une excellente efficacité des herbicides, ce qui est plus facile à obtenir avec des herbicides totaux associés à des variétés tolérantes (soja, mais aussi maïs) qu'avec les stratégies de désherbage classiques. Depuis le début des années 2000, la croissance des marges des grandes cultures (et en particulier celles du soja) est très supérieure à celle du coût de la vie, ce qui conforte la filière soja, accélère la déstructuration des filières animales, attire les investisseurs et favorise la concentration des terres. On assiste ainsi au développement d'une agriculture financière, basée sur la recherche du profit à court terme sur des surfaces cultivées de plusieurs centaines de milliers d'hectares, où toute 
référence à la gestion des rotations et de la fertilité des terres à long terme a disparu (pool de siembra). Avec l'accroissement des surfaces en soja et le raccourcissement des rotations, on assiste au développement de viroses et maladies fongiques, et à l'apparition de résistances au glyphosate, entraînant une augmentation de l'usage de pesticides. La sojisacion, dont les filières sont essentiellement tournées vers l'exportation, améliore les performances économiques de l'agriculture argentine, mais est critiquée pour les nuisances environnementales (Botta et al., 2011) et sociales (en particulier la déstructuration des dynamiques territoriales locales, Albaladéjo, 2012) qui lui sont associées.

\section{La production de coton en Thaïlande (d'après Castella et al., 1999)}

En Thailande, les surfaces cultivées en coton suivent depuis les années 60 une évolution cyclique : augmentation progressive des surfaces et de la production liée à une intensification par les intrants, suivie d'une forte chute des rendements, entrainant celle des surfaces, avant que ne reprenne un nouveau cycle, 10 ou 15 ans après le précédent. L'intensification porte notamment sur l'adoption de variétés « améliorées », très productives, mais moins tolérantes aux attaques d'insectes que les variétés traditionnelles, et sur l'utilisation des insecticides les plus modernes. Au fil du temps, avec la multiplication des surfaces et des traitements, une forte pression de sélection s'exerce sur les insectes parasites du coton, dont certains deviennent résistants aux insecticides. Après une phase d'augmentation des doses et du nombre de traitements, stratégie qui ne fait qu'accroître la sélection des sous-populations résistantes, les insecticides perdent toute efficacité, les populations d'insectes deviennent incontrôlables, et les rendements s'effondrent. La culture du coton est alors remplacée par d'autres productions, jusqu'à ce qu'une autre famille d'insecticides apparaisse, et soit diffusée. Castella et al. (op.cit) montrent que tous les acteurs qui œuvrent autour de l'agriculture concourent au succès du paquet technique «coton intensif » pendant la «phase d'exploitation » : les services de développement agricole, les pouvoirs publics (qui souhaitent accroître la production de coton), les firmes agro-chimiques. Les commerçants locaux, qui sont les seuls interlocuteurs de nombreux agriculteurs (ils leur vendent semences et pesticides, et achètent leur production) sont en position d'imposer le paquet technique aux paysans endettés. Le développement de la protection intégrée (IPM), seul moyen de sortir du cycle infernal, aurait supposé, selon Castella et al. (op.cit.), la mobilisation coordonnée de nombreux acteurs : pouvoirs publics, sélectionneurs, paysans, commerçants, chercheurs, agents de développement... Celle-ci tarde à se mettre en place, et depuis le début des années 2000, les surfaces en coton décroissent de manière continue, et sont aujourd'hui à un niveau très bas (moins de 10000 ha, contre 152000 au plus haut pic, en 1981).

\section{La production bananière dans les Antilles (d'après Clermont-Dauphin et al., 2003)}

Sur les sols volcaniques des hautes terres de Guadeloupe (Antilles françaises), la monoculture intensive de bananiers est pratiquée depuis les années 1980. Tous les 3 
à 5 ans, les bananiers sont replantés, sous forme de vitro-plants indemnes de nématodes, Les matières organiques issues de la bananeraie précédente sont enfouies par un labour. Des doses massives d'engrais sont épandues. De nombreux traitements herbicides, fongicides, insecticides, nématicides sont réalisés. L'étude de l'activité biologique des sols sur un échantillon de parcelles (dont certaines peu intensifiées, volontairement sur-représentées) montre que le labour favorise le développement des populations de nématodes, et au contraire réduit les populations de vers de terre (qui auraient un effet dépresseur sur les populations de nématodes, d'après Lavelle et al., 2004). Les nématicides employés pour circonvenir ces populations de bio-agresseurs apparaissent plus défavorables aux vers de terre et aux espèces de nématodes peu nuisibles qu'aux nématodes les plus nuisibles. Du fait de ce cercle vicieux, quelques années après la plantation, les populations de nématodes sont trop importantes et un nouveau labour est réalisé pour une nouvelle plantation. On retrouve des systèmes de production analogues dans les principales zones de culture de bananes d'exportation. Comme dans les autres exemples, l'organisation de l'amont et de l'aval des filières est très cohérente avec cette production normalisée, favorable à la satisfaction de marchés de masse. Cependant, aux Antilles, ce système de production est aujourd'hui totalement déstabilisé par l'ouverture du marché européen à des bananes produites dans des pays à bas coût de main d'œuvre, et par le constat des dégâts environnementaux liés à l'utilisation historique des pesticides (Blazy, 2011). La filière antillaise est donc en quête d'une évolution de ses pratiques, permettant une reconnaissance de sa spécificité sur les marchés. Dans le cadre du Plan Banane Durable, l'Institut technique tropical, le Cirad et les producteurs se sont engagés dans une démarche d'innovation pour la réduction de l'utilisation des intrants (Dorel et al., 2011 ; Blazy, 2011).

\section{Les marges de manœuvre pour agir sur des systèmes agricoles verrouillés}

Tous ces systèmes fortement utilisateurs d'intrants ont eu des processus d'évolution comparables : finalisés par la maximisation du résultat économique à court terme, ils se caractérisent tous par une spécialisation régionale, le raccourcissement des rotations (allant jusqu'à la monoculture), et un rôle clé assigné aux pesticides. Ils ont également des impacts écologiques et sociaux assez analogues. La biodiversité et les services écosystémiques qui lui sont liés sont partout en recul, tant du fait de l'homogénéisation des mosaïques paysagères que de l'emploi immodéré de pesticides. La spécialisation des territoires ne favorise pas le recyclage des éléments fertilisants, et ces systèmes contribuent au gaspillage de ressources. Au plan social, les impacts de l'usage des pesticides sur la santé des agriculteurs sont mis à l'agenda en France métropolitaine, mais aussi aux Antilles et en Asie ; et la réduction de l'emploi agricole lié à l'intensification par les intrants et la mécanisation vide les campagnes en Argentine, après les avoir vidées en France. Enfin, les systèmes agricoles du Bassin parisien et de la Pampa sont fortement interconnectés, ce qui fait que l'élevage intensif français a une part de responsabilité dans l'évolution des systèmes pampéens et de leurs impacts, de même que le soja de la Pampa contribue aux pollutions des systèmes aquatiques de l'Ouest de la France. 
Ces systèmes intensifs en intrants ont une grande cohérence agronomique ; que l'on veuille les rendre plus propres, moins monotones ou plus sobres, il ne suffira pas d'ajuster une ou deux pratiques, c'est toute leur logique qu'il faudra changer. Leur verrouillage est autant lié à leur forte cohérence interne qu'à la conjonction des stratégies des acteurs de l'amont, de l'aval et du conseil technique avec les politiques publiques. Dans certains cas, l'emballement du système (coton en Thailande ou banane aux Antilles) a conduit à une crise majeure, obligeant à des changements radicaux de mode de production. Ailleurs, les systèmes actuels sont solidement ancrés, et on ne les fera évoluer ni par des « Yaka », ni par un simple effort d'information. Pour conduire une telle évolution, il est indispensable de penser et d'agir de manière systémique, évitant de se laisser abuser par des solutions dont l'apparente simplicité masquerait des effets indirects difficiles à maîtriser. Souvenons-nous des pesticides, qui entre les années 1960 et 1990, ont fait partout figure de solution miracle : la recherche, abusée par l'efficacité de la lutte chimique contre les bio-agresseurs, n'a pas suffisamment investi dans les alternatives, dont aucune n'est aussi « performante » à elle seule, mais qui, combinées dans des stratégies de protection intégrée, offrent des solutions très prometteuses (Vanloqueren et Baret, 2009). Il s'agit de travailler, tant au niveau de l'agroécosystème qu'au niveau du système économique et social, sur différents leviers complémentaires : conception et proposition de systèmes de culture innovants basés sur la compréhension des processus agroécologiques ; réorganisation des programmes de formation et des canaux de diffusion d'information; politiques publiques incitatives, ciblées non seulement sur les agriculteurs, mais aussi sur les autres acteurs de l'agriculture et des territoires (de Schutter, 2010)...

Pour autant, si l'on peut s'accorder sur la nécessité d'une forte évolution des systèmes agricoles pour les inscrire dans le développement durable, il serait présomptueux de tenter de définir ce qu'ils devraient être. Nous ne proposerons donc pas ici un répertoire de systèmes techniques réputés « durables » ou « agroécologiques », qui pourrait laisser penser qu'il existe des «bonnes » solutions, à vocation universelle, que tout un chacun n'aurait plus qu'à mettre en œuvre. Nous nous attacherons plutôt à rappeler les principes d'une démarche d'ingénierie agro-écologique, visant à aider les acteurs à élaborer, individuellement ou collectivement, leurs propres solutions. Face à la diversité des situations, mais aussi aux incertitudes de l'avenir, nous proposons de travailler sur des outils et démarches pour aider les acteurs de terrain à faire évoluer leurs systèmes techniques, à les adapter aux caractéristiques précises de leur situation. Nous garderons comme fil directeur l'exemple des systèmes de grande culture intensive du nord de la France.

\section{" Outils et démarches pour la reconception des systèmes agricoles : quelques pistes de travail}

Comme le souligne de Schutter (2010), l'agroécologie offre un cadre interdisciplinaire pour développer, en partenariat étroit avec les acteurs concernés, une recherche sur la transformation des systèmes agricoles. Ce cadre qui a été explicité dans plusieurs articles de synthèse (Wezel et al., 2009 ; Francis et al., 2003) mobilise 
conjointement les sciences agronomiques, l'écologie et les sciences sociales, avec les savoirs empiriques locaux, pour concevoir des systèmes alimentaires durables. L'agroécologie est définie comme l'étude intégrative de l'écologie de la totalité du système alimentaire (Francis et al., 2003). Cette définition, soulignent ces auteurs, « encourage les chercheurs [...] à embrasser la complétude et la connectivité des systèmes, invite au regard sur l'unicité de chaque lieu et sur les solutions appropriées à ses ressources et à ses contraintes. Elle étend notre pensée au-delà des pratiques de production et des impacts environnementaux immédiats à l'échelle du champ et de la ferme ».

La reconception de systèmes agricoles, dans la voie de l'agroécologie, ne peut ignorer l'existence de tensions, parfois fortes, entre objectifs contradictoires : tension classique entre exigences économiques et environnementales ; tensions entre acteurs du territoire, ayant des objectifs divergents ; tensions entre les logiques individuelles d'acteurs et leurs conséquences au niveau des paysages (par exemple, la concentration du choix de tous les agriculteurs sur les cultures ou les variétés les plus rentables crée des espaces génétiquement homogènes, favorables à la propagation des épidémies). Il s'agira donc de proposer des manières de construire, à différentes échelles, des compromis entre ces objectifs divergents (Meynard et al., 2012). Un autre enjeu, tout aussi important est de faciliter l'adaptation des systèmes agricoles au changement climatique, qui pourrait se traduire par un accroissement des risques climatiques (températures élevées, sécheresse...) et parasitaires (déplacement vers les zones tempérées de parasites des régions chaudes). Le changement climatique remet en question les références des acteurs de l'agriculture, du développement agricole et des territoires, en interrogeant la pertinence des savoirs acquis dans les années antérieures. Il sera donc utile, dans ce contexte, d'aider les acteurs à reconstruire leur capacité à se projeter dans l'avenir, en combinant des connaissances situées acquises dans leurs territoires d'activité et des connaissances issues de modèles climatiques et agroécologiques proposés par les scientifiques.

La reconception des systèmes agricoles interpelle des acteurs variés, en fonction desquels sera organisé le plan de cette dernière partie :

- outils et démarches proposés aux agriculteurs et à leurs conseillers directs ;

- outils et démarches pour aider les acteurs des territoires (par exemple : agriculteurs, pouvoirs publics locaux, associations environnementalistes, résidents...) à explorer des scénarios de gestion concertée ;

- réflexions sur l'action publique visant à accompagner la transformation des systèmes agricoles et favoriser la concertation entre les acteurs des territoires.

\section{Outils et démarches adressés aux agriculteurs et à leurs conseillers techniques}

Les propositions de nouveaux systèmes techniques plus durables, issues de la recherche et du développement, et à destination des agriculteurs, sont nombreuses et variées : outils d'ajustement de la fertilisation, de l'irrigation ou des traitements phytosanitaires, systèmes sans travail du sol, variétés résistantes, lutte biologique et méthodes prophylactiques combinées dans des systèmes de protection intégrée contre les ennemis des cultures, associations de variétés et d'espèces permettant de 
limiter les intrants et d'améliorer leur efficience ; systèmes fourragers autonomes basés sur des prairies plurispécifiques riches en légumineuses.

Mais adapter les systèmes agricoles à la diversité des sols, des agro-écosystèmes et des exploitations agricoles suppose de proposer aux agriculteurs et aux techniciens des démarches pour construire leurs propres solutions. Meynard $(2008,2012)$ relève que les démarches de conception de systèmes agricoles innovants s'organisent en deux grandes familles : la conception de novo et la conception « pas à pas ». La conception de novo vise la construction de systèmes en rupture ; elle peut s'appuyer sur des ateliers de conception ou sur l'exploration in silico de systèmes techniques innovants à l'aide d'un modèle informatique (Rossing et al., 1997 ; Bergez et al., 2010). Dans la conception pas à pas, on ne cherche pas à créer une rupture, mais à organiser une transition progressive vers des systèmes innovants, en s'appuyant sur des boucles d'apprentissage. Ces deux familles de démarches sont complémentaires :

- la conception de novo ouvre le champ des possibles, en débridant l'inventivité, et permet ainsi d'explorer des solutions très innovantes, éventuellement incompatibles aujourd'hui avec le système sociotechnique, mais dont l'exploration contribue à préparer l'avenir ;

- dans la conception pas à pas, l'exploration est plus prudente, mais présente l'avantage de s'adapter plus aisément aux contraintes spécifiques de chaque situation agricole ; l'agriculteur, souvent accompagné par un technicien ou par un collectif de pairs, met au point son nouveau système, en même temps qu'il se convainc de son intérêt.

L'exemple d'une exploitation picarde dans laquelle les dynamiques d'apprentissage de la conception pas à pas ont été mobilisées pour réduire les intrants et les nuisances environnementales est développé dans l'encadré 4.1 (d'après Mischler et al., 2009). L'enjeu de la reconception, dans ce cas précis, a été d'inclure des critères environnementaux dans l'apprentissage des agriculteurs. En effet, chaque année, les agriculteurs tirent parti de leurs observations pour parfaire leur expérience. Ils observent les réactions des sols et des cultures, scrutent le comportement de la nouvelle variété qu'ils ont essayé et testent, dans un coin de champ, l'innovation conseillée par un technicien. Cependant, les critères sur lesquels ils s'appuient pour réaliser cette évaluation sont, le plus souvent, essentiellement liés à la production (rendement, qualité) et aux performances économiques de leur exploitation. Pour se lancer dans la reconception de leurs systèmes, les agriculteurs ont donc besoin que la recherche leur propose :

- une batterie d'indicateurs de diagnostic, aisément mesurables, et appropriables ; la mise au point d'indicateurs agro-écologiques est actuellement très active (Bockstaller et al., 2008, Sadok et al., 2008) ;

- une bibliothèque d'innovations (variétés, produits phytosanitaires, mais aussi outils d'aide à la décision, méthodes de protection intégrée, méthodes de gestion des matières organiques, cultures de diversification, exemples de systèmes de culture innovants conçus par des démarches de type de novo...). Il est important que chaque innovation ait été caractérisée de manière précise, pour aider l'agriculteur dans ses choix : temps de travail, matériel et compétences nécessaires, impacts attendus sur l'environnement et sur la production, effets systémiques sur d'autres pratiques... 


\section{Encadré 4.1. Conception «pas à pas » d'un système de production agroécologique en Picardie (Mischler et al., 2009)}

L'exploitation de M.X, agriculteur en Picardie, est spécialisée en grande culture (céréales, oléo-protéagineux, betterave à sucre). Elle est suivie dans le cadre du programme «Production intégrée » conduit par Agrotransfert Ressources et territoires, les Chambres d'agriculture de Picardie et l'Inra.

En 2002, un diagnostic agronomique et environnemental est réalisé par l'agriculteur et un conseiller. Un point faible majeur est mis en évidence : une utilisation importante de pesticides (indice de fréquence de pesticides IFT de plus de 8 - ce qui veut dire que l'agriculteur a effectué 8 traitements à dose homologuée, en moyenne sur les parcelles de son exploitation), sur des rotations peu diversifiées. Le technicien et l'agriculteur examinent alors ensemble les solutions envisageables : Quelles nouvelles cultures sont possibles ? Pour quels débouchés ? Sont-elles compatibles avec le matériel de l'agriculteur? Avec son organisation du travail ? Sur chacune des cultures, quel mode de conduite adopter pour limiter l'usage de pesticides ? Est-il possible de passer au désherbage mécanique ? Quelles nouvelles variétés ? Peut-on envisager des associations d'espèces ou de variétés ? Dans la "bibliothèque d'innovations » proposées par le conseiller (sur la base de son expérience et des acquis de la recherche), l'agriculteur fait son choix, en tenant compte de ses contraintes spécifiques; il essaie les innovations dans une ou deux parcelles; puis il les étend à l'ensemble de sa ferme. Chaque année, il fait le point avec son conseiller et avec un groupe d'agriculteurs engagés dans la même démarche : Quelles innovations ont donné satisfaction? Quels échecs ? Comment faire évoluer le plan d'action initial ?

Six ans après, il a diversifié ses rotations, changé ses variétés et ses modes de culture, amélioré son bilan énergétique (utilisation de moins d'engrais azoté et réduction du travail du sol) et réduit l'IFT moyen de son exploitation à 3 (variétés résistantes, rotations longues, désherbage mécanique). Le suivi effectué par le technicien montre que le temps de travail est un peu augmenté, mais que le revenu n'est pas affecté.

Les agriculteurs ne peuvent, le plus souvent, conduire seuls de tels changements, qui remettent en cause pratiques, savoirs, représentations sociales et organisation du travail. Les travaux des sociologues (Darré et al., 1994 ; Warner, 2007 ; Lamine et al., 2009) montrent le rôle des groupes d'échange entre agriculteurs dans l'apprentissage des systèmes innovants, à la fois source d'idées et de démultiplication des expériences, et soutien moral face à la prise de risque.

\section{Outils et démarches pour la concertation au niveau des paysages}

Une action au niveau des exploitations agricoles individuelles est évidemment insuffisante pour gérer les processus qui expriment leur effet au niveau des paysages (érosion, impacts sur la biodiversité, pollutions d'aquifères). Elle doit s'accompagner d'une concertation entre fermes voisines et, plus généralement, entre les différents acteurs du territoire, pour imaginer des mosaïques paysagères et des agencements spatiaux de systèmes agricoles (Papy et Torre, 2002 ; Soulard, 2005 ; Thenail et al., 2009). 
Comment favoriser une telle concertation ? Il s'agit d'une question complexe, car les intérêts des différents acteurs peuvent être contradictoires, leurs représentations de la situation inconciliables, ou leur information asymétrique ; certains peuvent ne retirer aucun bénéfice d'une coordination des actes gestionnaires, ou ne pas percevoir les bénéfices qu'ils pourraient en retirer ; les réseaux d'acteurs préexistants peuvent favoriser (mais aussi parfois entraver) les coordinations innovantes. Pour de telles questions, la recherche participative est particulièrement adaptée, car elle est favorable aux processus d'apprentissage collectifs, qui accompagnent la construction de normes collectives et la coordination des pratiques. Parmi les démarches participatives, l'Integrated assessment (Bland, 1999 ; Pahl-Wostl, 2005) et la modélisation d'accompagnement (companion modeling, collectif ComMod, 2006) reposent sur l'utilisation de modèles de simulation comme médiateurs entre les porteurs d'enjeu. Autour des résultats du modèle, s'organisent des comparaisons de scénarios, des débats, des concertations, qui amènent à faire évoluer les scénarios et à favoriser des convergences entre les parties prenantes (stakeholders). À titre d'exemple, la modélisation d'accompagnement est basée sur une approche séquentielle (Etienne et al., 2011) :

- construction d'une représentation partagée des processus territoriaux qu'il s'agit de piloter collectivement. Les chercheurs développent avec les parties prenantes un modèle informatique qui, en s'appuyant sur les connaissances des acteurs locaux, combinées avec celles des scientifiques, rend compte des interactions clef entre les systèmes écologiques, agricoles et sociaux ;

- organisation d'un jeu de rôle, qui, en mettant les acteurs en situation, leur permet d'appréhender, de l'intérieur, la complexité des interactions ;

- comparaison de scénarios construits sur la base du jeu de rôle ; les scénarios diffèrent par la stratégie adoptée par certains acteurs, ou par les réglementations, par exemple. Le modèle permet de peser les avantages et inconvénients des diffèrents scénarios, de les améliorer, et de se rapprocher ainsi de solutions plus satisfaisantes.

Le développement des recherches sur la modélisation des processus spatiaux constitue un atout précieux pour le test, avec les acteurs du territoire, de scénarios d'occupation des sols et d'évolution des systèmes agricoles. Agronomes et écologues doivent se mobiliser ensemble pour modéliser à cette fin les interactions entre systèmes techniques et agro-écosystèmes. Les difficultés méthodologiques d'une telle construction interdisciplinaire sont nombreuses : complexité de la traduction des actes techniques en variables écologiques ; points de vue différents également sur la temporalité des processus ; points de vue non concordants sur les découpages pertinents de l'espace (le paysage n'est réductible ni aux seules parcelles chères à l'agronome, ni aux seules « structures paysagères » considérées par les écologues du paysage).

\section{Quelques réflexions sur l'action publique}

L'action des pouvoirs publics sur l'évolution des systèmes agricoles et des paysages est potentiellement considérable. Pour revenir sur l'exemple du bassin de la Seine du début de cet article, dans le passé, le soutien des prix du blé a contribué à la 
disparition de l'élevage dans les zones favorables à la céréaliculture ; les primes au drainage ont encouragé la régression des prairies naturelles humides, et de leurs services écosystémiques (biodiversité, épuration des eaux...) ; la prime à l'irrigation, créée par la politique agricole des années 1990 a encouragé la multiplication des irrigants et la monoculture de maïs, avec des conséquences parfois graves sur l'état écologique des milieux aquatiques (Meynard et al., 2003). La spécialisation des cultures, le raccourcissement des rotations et le développement de systèmes de culture intensifs fortement consommateurs de pesticides ont ainsi été favorisés par les règles du jeu édictées par les pouvoirs publics.

Les instruments économiques mobilisables pour agir sur la gestion des agro-écosystèmes sont bien connus : aides publiques (mesures agri-environnementales, conditionnalité, soutien à la diffusion d'innovations) ; taxes (sur les pesticides, sur l'énergie...), obligations ou interdictions, quotas. Ces outils pourraient être mieux utilisés pour rémunérer, directement ou indirectement les services écosystémiques rendus par l'agriculture. Cependant, une vision insuffisamment systémique conduit parfois à des effets pervers : par exemple, l'obligation de couverture des sols en hiver, par le semis d'une « culture intermédiaire » (pour réduire les risques de pollution nitrique des eaux) empêche de pratiquer déchaumages et faux semis, techniques qui permettent de réduire les herbicides, et favorisent, dans certains milieux, la multiplication des limaces. De même, en Europe, la disparition annoncée pour 2015 des quotas laitiers va conduire inéluctablement à une concentration de la production chez les exploitants les plus compétitifs, et de la collecte dans les régions où la densité de producteurs est forte. La spécialisation des exploitations et des territoires ne pourra qu'en être accrue.

La promotion de la diversification des cultures via des règlementations ou un soutien financier des pouvoirs publics n'aura cependant un effet sur le long terme que si elle est pérennisée par les mécanismes du marché. La diversification des mosaïques paysagères, favorable à la biodiversité comme à la réduction des pesticides, passe par un soutien des pouvoirs publics à la construction de nouvelles filières : ils doivent aider des filières de diversification à émerger, à se consolider, à se crédibiliser. Ceci supposerait, entre autres, de conduire des actions coordonnées (i) au niveau de la sélection des espèces « orphelines » (rôle de la recherche publique ; soutiens ciblés à la sélection privée) ; (ii) au niveau de la R\&D agronomique (soutien ciblé à l'élaboration de références sur les cultures de diversification..) ; (iii) au niveau de l'aval des filières pour favoriser les innovations technologiques et les coordinations entre acteurs.

On a vu d'autre part, le rôle majeur des dynamiques d'apprentissages individuels et collectifs dans les transformations de l'agriculture. Comment les pouvoirs publics peuvent-ils contribuer à favoriser les dynamiques d'apprentissage ? Tout simplement en donnant la plus grande place, au niveau des incitations et des règlementations, aux démarches de type «management environnemental », de préférence aux démarches de normalisation des pratiques du type « code de bonnes pratiques ». Pour un agronome, les codes de bonnes pratiques agricoles, outil le plus souvent employé pour inciter les agriculteurs à adopter des pratiques vertueuses, constituent un contresens. Et ceci, pour plusieurs raisons : (i) ils visent à standardiser les pratiques, contraignant parfois fortement les capacités des agriculteurs à s'adapter à la 
diversité des sols, des climats et des situations agricoles ; (ii) ils sont codifiés au niveau de la technique agricole élémentaire, alors que les impacts environnementaux dépendent souvent d'interactions entre plusieurs techniques ; (iii) ils sont vécus comme des contraintes, dévalorisant ainsi aux yeux des agriculteurs la protection de l'environnement. L'obligation de résultats semble avoir des vertus pédagogiques que n'a pas l'obligation de moyens, car elle incite les agriculteurs à porter des diagnostics sur leur situation (comparaison entre le « résultat» obtenu, apprécié par un indicateur proposé par les pouvoirs publics, et le « résultat » réel) et les encourage à mettre en œuvre des boucles d'amélioration vertueuses (Meynard, 2010).

La mise en œuvre des politiques environnementales s'appuie de plus en plus souvent sur des démarches de concertation entre acteurs hétérogènes, qui visent, en situation d'incomplétude des connaissances, à adapter le cadre proposé aux spécificités des territoires. Mais ces dispositifs sont couteux et ne s'adressent qu'à des surfaces limitées. En complément de ces dispositifs, nous suggérons que l'action publique s'appuie sur le rôle de coordination des pratiques que pourraient jouer des entreprises ayant une vocation territoriale. Ainsi, en France, les coopératives agricoles, qui opèrent généralement sur des territoires clairement définis, de plusieurs milliers de $\mathrm{km}^{2}$, occupent un nœud de décision essentiel : elles vendent des semences aux agriculteurs et pourraient promouvoir les variétés résistantes aux maladies et des mélanges de variétés ou d'espèces. Elles diffusent un conseil très écouté et, par leur fonction de collecte, pourraient inciter à la diversification des cultures. Les politiques agri-environnementales, qui sont aujourd'hui surtout ciblées sur les agriculteurs devraient sans doute aussi s'intéresser à ces entreprises comme aux collectivités locales qui pilotent l'aménagement de l'espace.

\section{" Conclusion}

Dans son rapport à l'ONU sur le droit à l'alimentation, de Schutter (2010) souligne l'impérieuse nécessité de «faire référence à l'agroécologie et à l'agriculture durable dans les stratégies nationales pour la réalisation du droit à l'alimentation ». Il recommande pour cela de « réorienter les dépenses publiques vers l'agriculture en accordant la priorité à la fourniture de biens publics tels que les services de vulgarisation, les infrastructures rurales et la recherche agricole, en tirant parti des atouts complémentaires des méthodes de sélection génétique des semences et des variétés et des méthodes agroécologiques ». L'analyse des agricultures intensives en intrants et des voies d'évolution pour celles-ci, que nous venons de conduire, aboutit à des conclusions convergentes. Cependant, en mettant l'accent sur la cohérence des systèmes sociotechniques et sur les processus de verrouillage technologique, nous montrons que l'évolution souhaitée ne pourra réussir que si l'ensemble des acteurs concernés, au premier rang desquels les pouvoirs publics, pensent et agissent de manière systémique. Les séduisantes simplifications du type « 1 problème, 1 solution » ou « un objectif de politique publique, 1 instrument $»$ ont sans doute fait leur temps. Les pouvoirs publics ont dans ce cadre un rôle majeur à jouer pour mobiliser l'ensemble des acteurs. Différents instruments pourront être employés : instruments économiques classiques (fiscalité, quotas, interdictions, marchés de droits, subventions...), mais aussi soutien à la recherche et à l'innovation, aux apprentissages, à l'action 
collective et à la coordination des acteurs économiques... L'angle d'attaque que nous avons adopté, l'analyse des processus agronomiques et de leurs liens avec le système économique et social, conduit à une analyse convergente avec celle développée par d'autres auteurs de ce livre sur le concept de système d'innovation : c'est bien sur toutes les composantes du système socio-écologique qu'il est nécessaire d'agir pour engager et accompagner le changement en agriculture. Infléchir des dynamiques économiques et sociales à forte inertie, comme celles que nous avons décrites dans plusieurs régions du monde, supposera de mettre en œuvre sans tarder des politiques volontaristes... Mais aussi d'envoyer des messages clairs aux acteurs, pour qu'ils engagent les mutations nécessaires, ce qui nécessitera une constance des politiques publiques et une transparence dans l'évolution des réglementations. En France, aujourd'hui, l'un des obstacles au développement d'une agriculture durable n'est-il pas dans le fait que les politiques publiques ne sont pas elles-mêmes suffisamment durables (Meynard, 2010)?

\section{" Références bibliographiques}

Albaladéjo C., 2012. Les transformations de l'espace rural pampéen face à la mondialisation. Annales de géographie (sous presse).

Bergez J.E., Colbach N., Crespo O., Garcia F., Jeuffroy M.H., Justes E., Loyce C., Munier-Jolain N., Sadok W., 2010. Designing crop management systems by simulation. Europ. J. Agronomy, 32, 3-9. Bland W.L., 1999. Toward integrated assessment in agriculture. Agricultural Systems, 60, 157-167. Blazy J.-M., 2011. De l'innovation à l'adoption de nouvelles pratiques dans la filière banane. Innovations Agronomiques, 16, 25-37.

Bockstaller C., Guichard L., Makowski D., Aveline A., Girardin P., Plantureux S., 2008. Agri-environmental indicators to assess cropping and farming systems. A review. Agronomy for Sustainable Development, 28, 139-149.

Botta G.F., Tolón-Becerra A., Lastra-Bravo X., Tourn M.C., 2011. A Research of the Environmental and Social Effects of the Adoption of Biotechnological Practices for Soybean Cultivation in Argentina. American Journal of Plant Sciences, 2, 359-369.

Butault J.P., Dedryver C.A., Gary C., Guichard L., Jacquet F., Meynard J.-M., Nicot P., Pitrat M., Reau R., Sauphanor B., Savini I., Volay T., 2010. Ecophyto R\&D, Quelles voizs pour réduire l'usage des pesticides. Synthèse du rapport d'étude. Inra editions, France, 90 pages.

Castella J.C., Jourdain D., Trébuil G., Napompeth B., 1999. A systems approach to understanding obstacles to effective implementation of IPM in Thailand: key issues for the cotton industry. Agriculture, ecosystems and Environment, 72, 17-34.

Clermont-Dauphin C., Cabidoche Y.M., Meynard J.M., 2004. Effects of intensive monocropping of bananas on properties of volcanic soils in the uplands of the French West Indies. Soil Use and Management, 20 (2), 105,113.

Collectif ComMod (Companion Modelling, Cirad-Inra-IRD), 2006. La modélisation comme outil d'accompagnement. Natures, Sciences et Sociétés, 13 (2), 165-168.

Cowan R., Gunby P., 1996. Sprayed to death: Path dependence, lock-in and pest control. Economic Journal, 106 (436), 521-543.

Daniel K., Chatellier V., Chevassus-Lozza E., 2008. Localisation des productions agricoles dans l'UE. L'enjeu de l'évolution des politiques agricole et commerciale. Chambres d'Agriculture $\mathrm{n}^{\circ}$ 969, 24-27.

Darré J.P., 1994. Pairs et experts dans l'agriculture. Dialogues et production de connaissances pour l'action, Ed. Erès, Ramonville Saint-Agne, 227 pages.

David P.A., 1985. Clio and the economics of QWERTY. American Economic Review, 75 (2) 332337. 
Dorel M., Tixier P., Dural D., Zanoletti S., 2011. Alternatives aux intrants chimiques en culture bananière. Innovations Agronomiques, 16, 1-11.

Dosi G., 1988. Sources, procedures and microeconomics. Effects on innovation. Journal of economic literature, 26 (3) 1120-71.

Etienne M., 2010. Companion Modelling. A participatory approach to sustainable development, Éditions Quae, Versailles.

Fares M., Magrini M.B., Triboulet P., 2012. Transition agroécologique, innovation et effets de verrouillage : le rôle de la structure organisationnelle des filières. Cahiers Agricultures, 21 (1), 34-45.

Francis C., Lieblein G., Gliessman S., Breland T.A., Creamer N., Harwood R., Salomonsson L., Helenius J., Rickerl D., Salvador R., Wiedenhoeft M., Simmons S., Allen P., Altieri M., Flora C., Poincelot R., 2003. Agroecology: The ecology of food systems. Journal of Sustainable Agriculture, 22, 99-118.

Grosso S., 2011. Transformations du conseil agricole en région pampéenne argentine et recomposition de la profession "d'ingénieur agronome ", thèse, Université Toulouse le Mirail.

Labarthe P., 2010. Services immatériels et verrouillage technologique. Le cas du conseil technique aux agriculteurs. Economies et Sociétés, 44, 173-196.

Lamine C., Meynard J.-M., Perrot N., Bellon S., 2009. Analyse des formes de transition vers des agricultures plus écologiques : les cas de l'Agriculture Biologique et de la Protection Intégrée. Innovations Agronomiques, 4, 483-493.

Lamine C., Meynard J.M., Bui S., Messéan A., 2010. Réductions d'intrants : des changements techniques, et après? Effets de verrouillage et voies d'évolution à l'échelle du système agri-alimentaire. Innovations Agronomiques, 8, 121-134.

Lavelle P., Blouin M., Boyer J., Cadet P., Laffray D., Pham-Thi A.T., Reversat G., Settle W., Zuily Y., 2004. Plant parasite control and soil fauna diversity. C. R. Biologies, 327, 629-638.

Meynard J.M., Girardin P., 1991. Produire autrement. Courrier de la cellule Environnement de L'Inra, 15,1-19.

Meynard J.M., Dupraz P., Dron D., 2003. Grande culture. Dossiers de l'Environnement de l'Inra. Expertise Collective ATEPE, Agriculture, Territoire, Environnement dans les Politiques Européennes, 69-91.

Meynard J.M., 2008. Produire autrement : réinventer les systèmes de cultures. In : Systèmes de culture innovants et durables (Reau R., Doré T., eds), Editions Éducagri, 11-27.

Meynard J.M., 2010. Réinventer les systèmes agricoles : quelle agronomie pour un développement durable ?, In: Vers une société sobre et désirable (Bourg D., Papaux A., eds), Presses Universitaires de France et Fondation Nicolas Hulot, 342-363.

Meynard J.M., Dedieu B., Bos A.P., 2012. Re-design and co-design of farming systems. An overview of methods and practices, In : Farming Systems Research into the 21st century: The new dynamic (Darnhofer I., Gibon D., Dedieu B., eds), Springer, 407-432.

Mischler P., Lheureux S., Dumoulin F., Menu P., Sene O., Hopquin J.P., Cariolle M., Reau R., Munier-Jolain N., Faloya V., Boizard H., Meynard J.M., 2009. Huit fermes de grande culture engagées en Production Intégrée réduisent les pesticides sans baisse de marge. Le Courrier de l'Environnement, 57, 73-91.

Pahl-Wostl C., 2005. Actor based analysis and modelling approaches. The Integrated Assessment Journal, 5, 97-118.

Papy F., Torre A., 2002. Quelles organisations territoriales pour concilier production agricole et gestion des ressources naturelles? Etudes et Recherches sur les Systèmes Agraires et le Développement, 33, 151-169.

Rossing W.A.H., Meynard J.M., van Ittersum M.K., 1997. Model-based explorations to support development of sustainable farming systems: case studies from France and the Netherlands. Eur. J. Agron., 7, 271-283.

Sadok W., Angevin F., Bergez J.E., Bockstaller C., Colomb B., Guichard L., Reau R., Doré T., 2008. Ex ante assessment of the sustainability of alternative cropping systems: implications for using multi-criteria decision-aid methods. A review, Agronomy for Sustainable Development, 28, 163174. 
Schott C., Mignolet C., Meynard J.M., 2010. Les oléoprotéagineux dans les systèmes de culture : évolution des assolements et des successions culturales depuis les années 1970 dans le bassin de la Seine. Oléagineux, Corps gras, Lipides, 17 (5), 276-291.

de Schutter O., 2010. Rapport du Rapporteur spécial sur le droit à l'alimentation, Organisation des Nations Unies, Assemblée Générale du 20 décembre 2010, 23 pages.

Soulard C.T., 2005. La multifonctionnalité de l'agriculture en pratique : étude des relations entre exploitations agricoles et étangs de la Dombes. Cybergeo, European Journal of Geography, 319, 12 p. http://cybergeo.revues.org/6610 ; DOI : 10.4000/cybergeo.6610

Thenail C., Joannon A., Capitaine M., Souchere V., Mignolet C., Schermann N., Di Pietro F., Pons Y., Gaucherel C., Viaud V., Baudry J., 2009. The contribution of crop-rotation organization in farms to crop-mosaic patterning at local landscape scales. Agriculture Ecosystems and Environment, 131, 207-219.

Vanloqueren G., Baret P., 2008. Why are ecological, low-input, multi-resistant wheat cultivars slow to develop commercially? A Belgian agricultural 'lock-in' case study. Ecological Economics, 66, 436-446.

Vanloqueren G., Baret P., 2009. How agricultural research systems shape a technological regime that develops genetic engineering but locks out agroecological innovations. Research Policy, 38, 971-983.

Warner K.D., 2007. Agroecology in action; extending alternative agriculture through social networks, the MIT Press, Cambridge (USA), London (UK).

Wezel A., Bellon S., Doré T., Francis C., Vallod D., David C., 2009. Agroecology as a science, a movement or a practice. A review. Agronomy for Sustainable Development, 29 (4), 503-515. 



\title{
Chapitre 5 \\ Innovation et insertion sociale, réduire la vulnérabilité des populations rurales
}

\author{
Denis REQUIER-DESJARDINS
}

L'innovation est aujourd'hui au centre de la réflexion sur les trajectoires de développement et de croissance au Nord comme au Sud. Cette importance se manifeste notamment par la référence constante au développement de "l'économie de la connaissance », portée par un faisceau de technologies de l'information et de la communication et qui conduit à un bouleversement des «paradigmes technologiques » au moins aussi majeur que ceux qu'a pu connaître l'économie mondiale lors des premières révolutions industrielles.

La question de l'innovation concerne les pays du Sud, quel que soit leur niveau de développement : leur intégration à l'économie de la connaissance constitue un enjeu fondamental. Elle concerne également l'agriculture et l'agroalimentaire pour au moins trois raisons :

- l'agriculture et l'agroalimentaire utilisent essentiellement le vivant dans leur processus productifs, or les biotechnologies sont certainement un des secteurs d'innovation les plus dynamiques dans la période actuelle, avec un certain nombre de percées majeures dont les impacts ne sont probablement pas pleinement mesurés ; - l'agriculture et l'agroalimentaire, en raison justement de leur utilisation du vivant, ressentent de manière significative la contrainte de s'inscrire dans un processus de développement durable, façonnant les trajectoires d'innovation. Les enjeux autour de la biodiversité, du changement climatique de l'usage des sols, à la base des trois grandes conventions sur l'environnement, interpellent fortement l'agriculture ;

- la capacité de l'agriculture à nourrir une population mondiale en expansion (même si celle-ci est aujourd'hui globalement ralentie) apparait comme une condition forte de la durabilité, qui concerne notamment des populations fragilisées dont l'accès à l'alimentation n'est pas garanti. Ces populations sont souvent très dépendante de l'agriculture, la pauvreté dans le monde restant essentiellement rurale. La question du développement en ce début de millénaire est de plus en plus identifiée par la lutte contre toutes les formes de pauvreté, monétaire mais aussi de capacité, et les discriminations liées à la pauvreté, comme en témoigne la définition des Millenium Development Goals. 
Il est donc légitime de considérer la question de l'innovation dans l'agriculture et l'agroalimentaire du point de vue de sa capacité à réduire l'incidence de la pauvreté dans les zones rurales des pays du Sud et à favoriser l'émergence d'une trajectoire de développement durable, intégrant à la fois les dimensions environnementale mais aussi économique et sociale de la durabilité. C'est à ce questionnement, qui constituait un des hot topics du symposium ISDA, que cet article sera consacré.

Dans cette perspective, nous faisons l'hypothèse que la question de la contribution de l'innovation à la lutte contre la pauvreté dans les pays du Sud se pose différemment si on considère la réduction de la pauvreté des agriculteurs familiaux ou simplement la réduction de la pauvreté rurale. Si dans le premier cas on peut la poser comme la définition d'une forme d'innovation agricole qui puisse être prise en charge par les petits agriculteurs et leur permette d'améliorer leur revenu par leur plus grande efficacité productive, dans le second cas elle renvoie à l'ensemble des activités et des pratiques en zone rurale et au processus de décision au sein des ménages : elle n'est donc plus spécifiquement agricole. La définition de la durabilité des trajectoires de développement des zones rurales au sud s'en trouve modifiée. La question qui se pose alors est celle de l'articulation entre ces deux approches de l'innovation dans les zones rurales.

Nous aborderons cette question en trois temps :

- en premier lieu, nous opérerons un retour sur les concepts d'innovation, de pauvreté et de ruralité, qui soulèvent de nombreuses questions notamment en économie de l'innovation et économie du développement. La relation entre agriculture et ruralité est notamment aujourd'hui en débat ;

- en second lieu, nous nous concentrerons sur les processus d'innovation qui affectent l'agriculture et l'agroalimentaire ;

- enfin nous poserons la question des pratiques innovantes mises en œuvre par les ménages ruraux et plus largement de l'innovation dans les territoires ruraux.

En conclusion, nous poserons la question des politiques publiques, nationales et internationales, de lutte contre la pauvreté dans ce contexte.

\section{" Retours sur quelques concepts}

Nous revenons d'abord brièvement sur le concept d'innovation avant de traiter de la pauvreté et de son rapport avec l'inégalité, ainsi que de la ruralité et de son rapport avec l'agriculture.

\section{L'innovation}

Les premières réflexions sur l'innovation (Schumpeter, 1911) ont mis en évidence une typologie des innovations qui dépasse la simple innovation technologique, centrée sur les procédés de production : s'y adjoint en effet l'innovation produit, l'innovation organisationnelle et l'innovation de marché. Une réflexion sur l'innovation ne peut donc se limiter au cadre strict des processus productifs mais doit prendre en compte, même du point de vue de la discipline économique, les organisations, les institutions et les pratiques sociales. 
Dans une perspective «néo-schumpeterienne », l'économie du changement technique et en particulier les approches évolutionnistes (Nelson et Winter, 1982) ont mis en évidence la distinction entre innovation de rupture et innovation incrémentale, distinction qui renvoie aux notions de paradigme technologique et de trajectoire technologique. Les innovations de rupture créent un nouveau paradigme technologique, c'est-à-dire un ensemble de principes qui orientent la recherche de nouveaux procédés, et les innovations incrémentales viennent approfondir ce paradigme sans en remettre en cause les principes. Les innovations sont en effet dépendantes par rapport au sentier, chacune limitant les options futures et verrouillant la trajectoire technologique, qui seule peut être remise en cause par une innovation de rupture. Ces notions, au départ centrées sur les innovations technologiques, peuvent être étendues aux autres formes d'innovation, notamment aux innovations organisationnelles et institutionnelles ${ }^{1}$.

Ce courant d'analyse a aussi procédé à une extension du champ d'identification des acteurs de l'innovation. Schumpeter établissait une distinction entre invention et innovation. L'invention étant un processus hors marché en amont de l'innovation, et l'innovation consistant dans la valorisation économique d'une invention par un entrepreneur, acteur central de l'innovation. La phase ultérieure de diffusion de l'innovation n'était pas censée affecter le contenu de l'innovation. Pourtant l'économie évolutionniste réintègre l'invention dans le processus d'innovation et considère l'activité de recherche comme une activité économique. De ce fait, l'entrepreneur ou l'entreprise n'est plus le seul acteur de l'innovation qui devient alors un processus complexe présentant des boucles de rétroaction (Kline et Rosenberg, 1986) associant les utilisateurs de l'innovation, entreprises ou acteurs de la société civile, qui peuvent intervenir dans le processus notamment en générant des améliorations incrémentales. L'innovation apparaît désormais comme le produit de l'action collective à travers notamment l'identification de réseaux d'innovation, liés aux formes de la connaissance. «L'économie de la connaissance » fait référence à ces processus complexes : la connaissance est à la fois un bien non rival et un bien appropriable, notamment à travers les dispositifs de propriété intellectuelle ; elle est au surplus son propre input. Le système d'innovation est alors confronté à une tension entre la nécessité de créer et garantir des droits de propriété sur l'innovation pour assurer la rémunération du coût de l'innovation, et la nécessité de la diffuser le plus largement possible pour notamment bénéficier des boucles de rétroaction mentionnées ci-dessus et favoriser l'émergence de nouvelles innovations ${ }^{2}$. L'agriculture n'est évidemment pas à l'abri de ces processus : elle repose sur l'utilisation des processus du vivant et donc sur les biotechnologies, objet de conflit sur la propriété intellectuelle autour du «brevetage du vivant ». Plus généralement, les processus d'innovation en agriculture vont mettre en jeu des boucles de rétroaction impliquant des acteurs bien au-delà de l'agriculture stricto sensu.

1. North (1990) a notamment montré que, à l'échelle des nations, les institutions étaient dépendantes du sentier d'innovation institutionnelle, ce qui peut provoquer des phénomènes de verrouillage institutionnel. Cela remet notamment en cause la position qu'il avait défendue initialement d'une optimisation de la trajectoire institutionnelle dans le cadre du développement des économies de marché.

2. Les débats sur la propriété intellectuelle se font l'écho de cette tension, par exemple à travers la question de la durée des brevets ou de la possibilité de reconnaître une propriété intellectuelle collective. 


\section{Les approches de la pauvreté et des inégalités}

La pauvreté est traditionnellement approchée en référence à un revenu monétaire et à une « ligne de pauvreté » qui représente un revenu susceptible d'assurer la satisfaction d'un niveau de consommation considéré comme essentiel dans une société donnée. Toutefois cette approche, qui pose par ailleurs de nombreux problèmes de définition de la ligne de pauvreté (nationale ou internationale, absolue ou relative en pourcentage de la médiane des revenus par exemple), a été remise en cause par le développement de l'approche dite des « capacités » (capabilities), initiée par Sen (1987, 1999).

Si on en reste dans un premier temps à l'approche monétaire, la simple mesure de l'incidence de la pauvreté, c'est-à-dire de la proportion de la population en dessous de la ligne de pauvreté, indicateur le plus communément utilisé, doit être complétée par la prise en compte de l'intensité de la pauvreté, c'est-à-dire l'écart moyen à la ligne de pauvreté, ainsi que de la profondeur de la pauvreté, c'est-à-dire la répartition de la pauvreté parmi les pauvres. Leur prise en compte amène à s'intéresser en particulier à l'extrême pauvreté et aux effets de sélection entre les pauvres. Cet aspect est d'autant plus important que les pauvres sont non seulement l'objet de politiques spécifiques de lutte contre la pauvreté, mais sont affectés de plus par la dimension dite pro-poor des politiques sectorielles ou macro-économiques où la lutte contre la pauvreté est un «produit joint »d'une politique spécifique. De telles politiques peuvent en effet exercer des effets de sélection parmi les pauvres. Leurs impacts dépendent naturellement largement de la position des pauvres en termes d'intensité et de profondeur de la pauvreté.

Toutefois la mesure strictement monétaire de la pauvreté, sur laquelle repose la définition des indicateurs que nous venons d'évoquer, a été largement remise en cause comme approche exclusive de ce phénomène. D'une part dès le début des années 1980, l'approche dite des « besoins essentiels » identifie la pauvreté par le manque d'accès direct à un certain nombre de biens, en général avec un fort caractère de biens publics et en partie hors marché (eau, santé, hygiène, éducation, etc.) : le niveau d'accès est alors appréhendé par des indicateurs sociaux du développement qui ne renvoient pas à une mesure monétaire. D'autre part, l'approche en termes de capacités ou de « capabilités » (un néologisme inspiré du terme anglais capabilities) prolonge et amplifie celle des besoins essentiels. Plutôt que d'évaluer les situations personnelles en termes de «bien-être », défini comme la satisfaction matérielle à un moment donné, cette approche s'appuie sur le concept de good life, compris comme un projet de vie inscrit dans sa dynamique qui assure à la personne l'estime de soi et la reconnaissance sociale ainsi que la conformité à ses valeurs. Elle insiste dans cette perspective sur la capacité de choix de vie des personnes, qui dépend de leurs «fonctionnements de base », en particulier la santé, l'éducation, la culture, la sécurité, mais aussi la garantie d'un certain nombre de libertés fondamentales d'association et d'expression qui garantissent la reconnaissance de la personne au sein de la société.

Par ailleurs, la relation entre pauvreté et inégalité fait l'objet de nombreuses réflexions, mettant en évidence qu'il s'agit de deux variables différentes même si elles sont profondément articulées : les inégalités renforcent la perception subjective 


\section{Encadré 5.1. Horticulture et approche par les capacités au Cameroun Laurent Parrot, Philippe Pédelahore, Hubert De Bon et Rémi Kahane}

L'approche par les capacités permet de caractériser les transformations qui traversent le monde rural en Afrique Subsaharienne en décrivant par exemple les liens entre l'émergence du secteur rural non agricole et les trajectoires des migrants. Il s'agit alors de connaître les facteurs qui donneront les moyens aux personnes d'atteindre les styles de vie de leur choix.

Selon Dubois et Mahieu (2002), la construction de ces capacités dépend de trois facteurs : les biens et les potentialités possédés qui comprennent les disponibilités en capital, les actifs divers, les relations sociales, les croyances, etc. ; les caractéristiques personnelles ; et enfin, les opportunités sociales et notamment la position des femmes dans les sociétés.

Ces trois facteurs ont été étudiés pour mieux comprendre au Cameroun l'émergence de l'horticulture comme activité agricole principale auprès de ménages localisés dans une zone périurbaine à Muéa, à $40 \mathrm{~km}$ de Douala.

Nous avons montré que l'activité horticole nécessite divers actifs tels que l'accès à la terre et au crédit pour acquérir des intrants, mais aussi un accès aux réseaux sociaux informels pour accéder à la connaissance technique et à l'information sur les marchés. Ces éléments expliquent sans doute pourquoi les horticulteurs sont en moyenne plus âgés que leurs homologues producteurs vivriers. Il existe d'ailleurs un lien entre la pratique de l'horticulture et la diversification dans des activités rurales non agricoles : les ménages s'appuyant sur ces activités pour investir dans des systèmes horticoles.

Enfin, nous avons mis en évidence la prépondérance des groupements de femmes spécialisées en horticulture grâce à leurs réseaux sociaux particulièrement efficaces pour l'accès au crédit et à l'information.

Ces différents actifs se révèlent nécessaires pour atteindre une forme de transformation agricole révélant le passage d'une forme d'agriculture traditionnelle essentiellement vivrière basée sur peu d'intrants et de faibles niveaux de productivité et de rendements (agriculture de subsistance) à une forme adaptée aux contraintes des zones urbaines et péri-urbaines où les processus d'intensification nécessitent des connaissances particulières (agriculture commerciale). Ainsi, la transition urbaine pourrait agir positivement en permettant à des jeunes de migrer en ville dans un premier temps, d'accumuler suffisamment de capital et un certain esprit d'entreprise, puis de revenir avec à l'esprit de nouveaux systèmes de production ou de commercialisation agricole.

Cette approche intégrée dans l'espace et dans le temps illustre l'importance de politiques d'appui cherchant à mieux valoriser les interactions positives entre les secteurs urbain et rural dans le cadre d'un développement intégré (Parrot et al., 2010).

de la pauvreté mais les effets d'entraînement des dépenses des mieux lotis peuvent parfois réduire la pauvreté, alors qu'en revanche certaines mesures de réduction des inégalités peuvent parfois influer défavorablement sur la croissance économique et donc sur le niveau de vie général de la population. La solution rawlsienne (Rawls, 1971) du « voile d'ignorance » vise à résoudre théoriquement cette contradiction en mettant au premier plan la priorité « lexicologique » des libertés fondamentales et en faisant de l'amélioration de la situation des plus défavorisés un critère qui permet d'accepter certaines formes d'inégalité. 
Enfin, en termes de politiques de lutte contre la pauvreté, l'importance du ciblage, notamment en direction des plus pauvres, a été soulignée. Cette préoccupation, qui renvoie à la différenciation des situations de pauvreté mise en évidence ci-dessus, peut parfois rentrer en contradiction avec un principe général qui préconise la mise en ouvre de politiques économiques dites pro-poor. En effet, celles-ci considèrent que la lutte contre la pauvreté découlera des autres résultats visés, sans en faire un objectif central. La conséquence peut être l'existence d'effets pervers de ces politiques qui renforcent les effets de sélection entre les pauvres, en permettant certes parfois une réduction de l'incidence de la pauvreté mais sans en réduire l'intensité ou la profondeur ${ }^{3}$.

De telles considérations peuvent donc mettre en cause le caractère équitable des politiques pro-poor, alors même que leur but est justement d'améliorer l'équité au sein des sociétés concernées.

\section{L'agriculture, l'agroalimentaire et la ruralité}

S'interroger sur l'innovation en agriculture en tant que secteur productif suppose que l'on puisse donner une définition sans ambiguïté de ce secteur. Deux réponses à cette question sont traditionnellement données :

- l'agriculture utilise les processus du vivant pour produire essentiellement des produits agroalimentaires et accessoirement des matières premières industrielles ; - elle est essentiellement pratiquée dans des zones rurales, de faible densité, en raison de leur rapport spécifique à l'espace et à l'usage de la terre.

Ces deux définitions conduisent en fait à considérer l'insertion de l'agriculture dans des ensembles qui l'englobent.

En premier lieu, l'agriculture est fortement intégrée à un secteur agroalimentaire qui constitue une " chaîne de valeur globale " ${ }^{4}$, essentiellement dominée par les acteurs de l'aval, aujourd'hui les acteurs de la grande distribution. La question de l'innovation doit alors être posée à l'échelle du secteur agroalimentaire dans son intégralité et non simplement à celui de l'activité agricole stricto sensu dans la mesure où les effets de réseau et les rétroactions déjà mentionnées vont articuler les différents maillons de cette chaîne de valeur, depuis la production agricole jusqu'à la distribution au consommateur, en intégrant non seulement les différentes phases de la transformation mais aussi l'agrofourniture. Des travaux récents ont par exemple insisté sur l'impact des innovations dans l'ensemble de la chaîne et notamment du développement de la normalisation sur les producteurs agricoles des pays du Sud et en particulier les petits producteurs (Reardon et al., 2009).

En second lieu, l'agriculture renvoie à la ruralité, c'est-à-dire en s'en tenant à une approche économique, aux systèmes d'activités productives pratiquées dans les zones rurales. Les deux termes ont pu même être considérés comme synonymes dans la mesure où la production agricole apparaissait comme une caractéristique

3. On notera que s'interroger sur l'effet des politiques d'innovation sur la réduction de la pauvreté revient à s'interroger sur un mécanisme de ce type.

4. Au sens de Gereffi (1999) et Gereffi et al. (2005). 
essentielle de la ruralité. Pourtant, si pendant longtemps la ruralité d'un territoire a été connotée par l'emprise de l'agriculture comme activité économique dominante, ce qui permettait d'ailleurs d'assimiler les ménages ruraux à « l'agriculture familiale », on voit s'affirmer aujourd'hui un double décalage entre agriculture et ruralité. D’une part on assiste à la montée des activités et de la part des revenus hors exploitation, en grande partie non agricoles, dans l'ensemble des zones rurales et en particulier au sud (Haggblade et al., 2010 ; De Grammont, 2010). Cette diversification se manifeste d'abord à l'échelle du ménage, qui sera ainsi conduit à développer une stratégie de portefeuille vis-à-vis de cet ensemble d'activités dans le but de minimiser les risques qui pèsent sur les « moyens d'existence » du ménage (Ellis et Freeman, 2005). Certains considèrent que l'agriculture peut n'occuper dans ce portefeuille qu'une place résiduelle, voire être une variable d'ajustement (Rigg, 2006). La résultante de ces situations des ménages au niveau méso-économique des territoires ruraux peut être la consolidation d'une base économique "résidentielle », reposant sur les effets multiplicateurs de revenus d'origine externe dépensés localement (tourisme, migration, transferts divers). Cette base peut parfois se substituer à une base " productive ", résultant de l'exportation de biens et de services en dehors du territoire, cette dernière étant essentiellement agricole dans les territoires ruraux. Les effets d'entraînement vont alors stimuler le développement d'activités « domestiques » (Davezies, 2008), généralement non agricoles. D’autre part, et c'est un aspect peut-être jusqu'à présent moins souligné, on voit apparaître des formes d'agriculture, que l'on peut qualifier d'agriculture d'entreprise ou de firme, dans lesquelles l'activité agricole a adopté le même modèle organisationnel que de nombreux secteurs industriels, caractérisé d'une part par la financiarisation de l'activité et l'intervention d'acteurs financiers, et d'autre part la maîtrise d'un paquet technologique articulé sur le développement de l'économie de la connaissance, qui permet notamment la généralisation de la sous-traitance et la mise en place d'une « agriculture en réseau » (Hernandez, 2008 ; Clasadonte, 2010). Dans ce type de modèle, la terre, qui traditionnellement définit l'exploitation agricole et l'identité des agriculteurs, devient un intrant générique parmi d'autres, qui peut faire l'objet d'arbitrages globaux, ce qui déconnecte largement l'entreprise agricole de son inscription territoriale et donc de la ruralité.

Ces éléments aboutissent à configurer une « nouvelle ruralité » dans laquelle le revenu des ruraux ne dépend plus exclusivement, ni même principalement, des revenus de l'exploitation agricole et où l'agriculture relâche son lien au territoire. Compte tenu de cette déconnexion, la question est donc de savoir si des innovations dans l'agriculture ont un impact sur la pauvreté des ménages ruraux définie de la manière la plus extensive possible. Ne faut-il pas aborder directement la question de l'innovation dans les zones rurales, sans passer par la question de l'innovation en agriculture ? Une telle problématique renvoie également à la typologie des innovations, dans la mesure où l'innovation en agriculture aura un contenu technologique important alors que les territoires ruraux peuvent voir l'émergence d'innovations plutôt sociales ou organisationnelles. Si on s'intéresse à l'impact des innovations sur la réduction de la pauvreté rurale, on ne peut donc se limiter aux innovations qui affectent le secteur productif agricole : il faut prendre en compte l'ensemble des processus d'innovation qui affectent les économies et les sociétés rurales. 
Dans ce chapitre, nous évaluerons d'abord dans quelle mesure l'innovation agricole peut être un facteur de réduction de la pauvreté avant d'aborder la question des capacités d'innovation dans les territoires ruraux et de leur impact sur la pauvreté.

\section{" L'innovation agricole est-elle un facteur de réduction de la pauvreté et de la vulnérabilité, et de durabilité des trajectoires de développement?}

L'innovation agricole a été particulièrement dynamique durant les dernières décennies, comme en témoigne l'augmentation importante des rendements et, dans certaines régions, de la production par actif agricole, même si cette augmentation tend à se ralentir 5 .

En grossissant les traits, on peut considérer qu'il existe aujourd'hui deux trajectoires d'innovation, relativement antinomiques même si elles peuvent se rejoindre dans certains contextes, du fait des effets de verrouillage (lock-in) qui les affectent, cette opposition renvoyant à une vision différente de l'avenir de l'agriculture.

La première trajectoire, que l'on peut qualifier d'intensification productiviste, se situe dans le prolongement des innovations agricoles proposées dans les années 1970 aux agricultures du Sud autour de la révolution verte, c'est-à-dire l'usage de semences sélectionnées, la mécanisation et l'utilisation d'intrants agrochimiques (engrais et produits de traitement) dans le cadre d'exploitations privilégiant la spécialisation et donc la monoculture. Cette trajectoire est aujourd'hui fortement marquée par le progrès des biotechnologies, notamment dans le domaine des semences, avec l'apparition et le développement des organismes génétiquement modifiés, qui permettent notamment de diminuer le coût sur les grandes cultures en renforçant les économies d'échelle en matière de traitement phytosanitaire (semences tolérantes au round-up) ou de travail du sol (généralisation du semis direct). Une telle trajectoire a un impact important sur l'évolution de l'organisation de la production, dans la mesure où elle favorise les économies d'échelle ${ }^{6}$, alors que justement la faiblesse des économies d'échelle en agriculture a été l'argument souvent avancé pour expliquer la prédominance des structures d'agriculture familiale, notamment du fait des coûts de transaction liés à l'utilisation d'une main d'œuvre salariée (Lipton, 2006) et du faible taux d'utilisation du capital physique. «L'agriculture en réseau » mentionnée plus haut permet aujourd'hui de diminuer ou de sous-traiter les travaux agricoles intensifs en main-d'œuvre et de mettre en valeur des superficies très étendues. Elle peut être considérée comme une forme d'aboutissement de cette trajectoire.

5. Selon la prospective Agrimonde (Paillard et al., 2010), la production de Kcal/j par ha aurait été multipliée par 2,5 entre 1961 et 2003, la croissance ayant été particulièrement forte en Asie. Dans la zone OCDE, la production alimentaire par actif a été multipliée par 7, mais l'évolution a été beaucoup plus lente pour les autres régions.

6. L'organisation de l'agriculture en réseau et des «pools de siembra » dans le cône sud de l'Amérique latine est l'illustration la plus évidente de cette augmentation de l'échelle de la production (Hernandez, 2008). 
Dans le cadre de cette trajectoire d'intensification, la durabilité environnementale ne peut guère que reposer sur une approche "ségrégationniste ", qui considère que la question de la protection des écosystèmes peut être réglée indépendamment de l'agriculture notamment par la mise en place d'aires protégées, vides de toute activité agricole.

Les innovations proposées aux petits agriculteurs et à l'agriculture familiale des pays du Sud se sont longtemps inscrites dans ce paradigme : il s'agissait notamment de favoriser l'accès des petits agriculteurs aux intrants et à la mécanisation, notamment en les subventionnant. Cependant, on insistait dans ce cadre sur le fait que les innovations doivent toucher en priorité les cultures vivrières, ce qui conduisait à déplorer par exemple le manque de recherches faites dans le domaine des céréales et tubercules traditionnels. On considère également que les innovations doivent être adaptées aux petites exploitations qui correspondent en gros à l'agriculture familiale, puisqu'elle constitue la majeure partie de la population active agricole et que les rendements y sont généralement faibles, donc avec un fort potentiel d'amélioration. Ainsi, l'innovation peut avoir un impact immédiat sur le revenu d'exploitants qui sont les premières victimes de la pauvreté. Dans ce but, on a privilégié par exemple l'appui à des politiques de vulgarisation, associées à des politiques d'accès aux « marchés manquants » (crédit, intrants, etc.) et aux marchés de commercialisation de leurs produits (infrastructures de transport). Les innovations techniques devaient être complétées par des innovations organisationnelles et des investissements en infrastructures permettant un meilleur accès aux marchés et donc l'augmentation de l'échelle de production. Pour certaines productions pour l'exportation, notamment dans le domaine des exportations non traditionnelles (fruits et légumes, fleurs) des formes d'agriculture contractuelle basées sur la maîtrise de l'irrigation, l'utilisation des intrants et le respect de normes de qualité définies par les acteurs dominants des filières agroalimentaires ont pu aussi concerner les agriculteurs familiaux.

Mais le bilan de cette intensification proposée aux petits agriculteurs a pu apparaître décevant (Dethier et al., 2011). Au mieux, elle génère des effets de sélection : seule une minorité de petits agriculteurs peut s'intégrer à ces systèmes et éventuellement développer une montée en gamme (upgrading) de leurs fonctions et de leurs compétences.

La deuxième trajectoire, qui se présente comme un paradigme de rupture, découle des interrogations sur l'impact environnemental des pratiques agricoles, et notamment des pratiques agricoles intensives en intrants et très spécialisées, portées par la première trajectoire. Elle se situe donc dans le débat sur le caractère multifonctionnel de l'agriculture et sur sa capacité à « gérer les écosystèmes cultivés » en les préservant. Elle propose une intensification «agro-écologique » qui privilégie la polyculture plutôt que la monoculture, les engrais naturels plutôt que les engrais chimiques, la lutte biologique plutôt que le recours à des produits de traitement. Elle propose des innovations, notamment en termes d'assolement et d'association de cultures, qui préservent la capacité des écosystèmes à se reproduire durablement, tout en permettant d'augmenter la production alimentaire, en minimisant son coût environnemental, au plan de l'activité agricole. Ces innovations peuvent d'ailleurs être associées à des innovations aux autres stades des filières agroalimentaires, qui réduisent l'impact environnemental par exemple en minimisant les coûts 
de transport en tentant de développer des « filières courtes » de commercialisation reposant sur des accords entre producteurs et consommateurs. Cette trajectoire renvoie à la « révolution doublement verte » (Conway, 1997 ; Griffon, 2006), qui se substituerait à l'approche des années 1970 à la « révolution verte » pour le développement de l'agriculture des pays du Sud.

Bien que ces innovations puissent être prises en charge dans le cadre de grandes exploitations, cette trajectoire d'innovation concerne plus particulièrement les formes d'agriculture familiale, c'est-à-dire des agriculteurs qui sont a priori plus susceptibles d'être affectés par la pauvreté ou simplement la vulnérabilité de leurs systèmes d'actifs. En effet, cette trajectoire s'appuie sur des éléments (associations et rotations des cultures, utilisation de variétés traditionnelles adaptées) qui font partie du patrimoine cognitif des agricultures traditionnelles. On est donc en présence d'une nouvelle formulation de la thèse de l'avantage comparatif de l'agriculture familiale, moins basée sur les coûts de transaction liés à l'usage de la main-d'œuvre salariée et plus sur son adaptation aux contraintes d'une agriculture multifonctionnelle et respectueuse de l'environnement.

En ce qui concerne l'impact sur la pauvreté et la vulnérabilité des populations pauvres des zones rurales ou urbaines des pays du Sud, le premier effet attendu des innovations d'intensification en agriculture est l'augmentation de la productivité agricole, donc des rendements. Ce renforcement des capacités productives des petits producteurs est un facteur de réduction de leur exposition à la pauvreté dans la perspective des « trois voies de sortie de la pauvreté » posées par la Banque mondiale (WDR, 2008). Par ailleurs, elle doit permettre en toute logique une augmentation des disponibilités alimentaires. La question qui doit cependant être posée est de savoir si en soi une telle évolution garantit l'amélioration de la sécurité alimentaire des populations concernées, en particulier dans les pays du Sud (Dethier et al., 2011).

En effet, ce type d'approche se concentre essentiellement sur la relation entre la production et les disponibilités alimentaires. Elle a été remise en cause dès les années 1980 dans le débat sur les bases de la « sécurité alimentaire »: la critique que fait Sen (1982) de l'hypothèse FAD (Food Availability Decline) dans l'explicitation des causes des famines et des crises alimentaires a joué un rôle fondamental dans ce changement de paradigme. Ce changement aboutit à souligner l'importance des « droits à l'alimentation ». L'augmentation de la production n'est pas en soi une garantie de sécurité alimentaire des ménages ; c'est leur capacité à accéder à l'alimentation par les échanges de droits qui est cruciale et l'insécurité alimentaire est un des aspects de la vulnérabilité des ménages en général.

Cependant, la référence à la souveraineté alimentaire peut faire du niveau de productivité agricole sur un territoire, notamment lorsque l'on raisonne à l'échelle nationale, un facteur essentiel de sa sécurité alimentaire dans la mesure où elle peut permettre de limiter les variations sur les prix, notamment pour l'approvisionnement des zones urbaines. La proximité entre producteurs et consommateurs, par exemple dans le cas de «filières courtes », proximité géographique et parfois organisationnelle, est également un facteur de réduction des risques, à la fois pour les consommateurs et pour les producteurs. Dans ce cas, la sécurité alimentaire locale des ménages dépend effectivement des capacités de production locale et du degré d'organisation des producteurs agricoles. Les innovations organisationnelles dans 
les filières agroalimentaires, notamment celles qui permettent de renforcer les liens entre producteurs et consommateurs, peuvent alors être considérées comme des facteurs renforçant l'effectivité des «droits à l'alimentation». De telles innovations organisationnelles sont cohérentes avec une trajectoire d'innovation privilégiant la durabilité environnementale autour des innovations agro-écologiques.

Par ailleurs, on doit considérer non seulement les innovations agricoles mais également les innovations dans l'ensemble des chaînes de valeur agroalimentaires. Or une abondante littérature met l'accent sur la transformation de la gouvernance de ces filières dans le cadre de la globalisation et leur impact sur l'agriculture familiale, avec l'émergence d'une part de la domination des supermarchés dans les pays en développement et d'autre part de grossistes spécialisés sur des produits spécifiques approvisionnant les centrales d'achat des supermarchés (Reardon et Timmer, 2005 ; Reardon et al., 2009). En conséquence, les marchés domestiques sur lesquels sont présents les agriculteurs familiaux sont très souvent en fait des marchés déjà globalisés et fonctionnant sur la base d'innovations techniques et organisationnelles telles que l'utilisation des technologies de l'information ou la gouvernance par les normes de qualité et de conditionnement qui s'imposent à ces agriculteurs. Certains agriculteurs familiaux peuvent s'intégrer à ces chaînes de valeur mais l'effet de sélection entre agriculteurs est important, accentuant le décalage entre un groupe de producteurs agricoles, souvent sous contrat, alors que d'autres, ayant souvent recours à des filières de commercialisation traditionnelles en déclin (grossistes, transporteurs et marchés de gros par exemple) peuvent voir leur activité de production agricole marginalisée.

Cette trajectoire d'innovation en agriculture et en agroalimentaire met en évidence des caractéristiques propres à l'innovation dans les autres secteurs, notamment l'importance des réseaux d'innovation, de l'action collective et des processus d'exclusion autour de l'innovation.

La littérature souligne que les conditions de l'innovation peuvent être particulières en agriculture, notamment parce que le secteur se prête moins bien aux économies d'échelle que d'autres et parce qu'il repose sur les mécanismes du vivant et sur un travail d'accumulation des connaissances opéré par des générations d'agriculteurs. Il semble pourtant que l'agriculture soit également de plus en plus le siège de processus d'innovation finalement assez comparables à ceux que l'on rencontre dans les autres secteurs. Par exemple de nombreux travaux s'intéressent au rôle des réseaux dans les processus d'innovation, notamment dans les « clusters » et il est significatif que des travaux fondateurs pour l'analyse de l'ensemble des secteurs, aient pris des exemples agricoles (Giuliani, 2007, sur le cluster viticole de Colchagua par exemple pour ce qui est de l'analyse des réseaux ou Porter, 1990, sur le California wine cluster).

La question des réseaux d'innovation renvoie à celle de l'action collective en matière d'innovation en agriculture, mais également à la question de l'appropriation des innovations et notamment à celle de la propriété intellectuelle. Deux aspects sont particulièrement important en agriculture, l'existence de brevets autour de la «brevetabilité du vivant » et accessoirement celle de leur durée de protection, et celle de la reconnaissance d'une propriété intellectuelle collective autour des connaissances traditionnelles pour les populations concernées. On retrouve cependant une interrogation générale : comment arbitrer entre la nécessaire valorisation des dépenses 
d'innovation par les rentes liées à l'innovation, et la non moins nécessaire diffusion des innovations ? En effet, les processus d'exclusion autour des réseaux d'action collective sont à la fois les garants de l'efficacité de cette action mais aussi un facteur d'inégalité et d'exclusion comme le montre parfois dans les pays du Sud l'exemple des signaux de qualité.

En conclusion, l'innovation intensificatrice en agriculture risque de renforcer les effets de sélection et de contribuer à la marginalisation de certains secteurs de la population rurale, en particulier de petits producteurs agricoles. On ne peut évidemment pas négliger l'impact que ces processus de sélection peuvent avoir sur la réduction de l'incidence de la pauvreté rurale en permettant l'amélioration du niveau de vie de nombreux agriculteurs familiaux. Toutefois, la question reste posée de leur impact sur l'intensité et la profondeur de la pauvreté, compte tenu des effets de marginalisation de ceux qui ne peuvent s'intégrer aux réseaux d'innovations. Il faut également tenir compte du fait qu'il existe d'autres voies de sortie de la pauvreté rurale, notamment par la diversification des activités des ménages ruraux. La question de la constitution des réseaux et de leur territorialité, tout autant que celles des accords locaux entre producteurs et consommateurs, et de l'organisation des producteurs peut permettre de reposer la question du lien entre agriculture et ruralité à travers leur référence au territoire.

Par ailleurs, les innovations agro-écologiques proposées aux producteurs agricoles sont souvent portées par des organisations dans le cadre de la «coopération par projets » et définies par les experts ou les techniciens. Elles ne correspondent pas forcément au système de valeurs ou aux pratiques des populations concernées (Dietsch, Ruault, 2010) et il n'est donc pas toujours évident qu'elles soient facilement adoptables par les petits agriculteurs. D'une manière générale, les innovations proposées portent essentiellement sur l'agriculture et partent du principe que la seule voie de sortie est le renforcement des capacités de production agricole. La sélection des leaders pour la promotion de ces innovations reproduit dans une certaine mesure les effets de sélection des dispositifs d'innovation de la trajectoire dominante.

Il faut peut-être alors se poser la question des innovations qui peuvent cibler directement la réduction de la pauvreté rurale, innovations qui ne sont alors pas obligatoirement agricoles. En effet, le développement d'activités non agricoles dans les zones rurales est également susceptible de diminuer l'impact de la pauvreté par la création de revenus. Ces activités peuvent d'ailleurs être également liées à la migration qui peut apparaître comme une "voie de sortie de la pauvreté » (Banque mondiale, RDM, 2008). Plus largement, l'innovation peut être vue comme un processus par lequel un groupe humain utilise la connaissance produite par différents agents pour créer une valeur nouvelle (Fernandez-Baca et al., 2010). Appliqué à une communauté rurale, cela étend largement le champ de l'innovation au-delà de la simple activité agricole.

Une condition qui peut permettre de sélectionner les innovations permettant de réduire la pauvreté rurale peut être la mise en place d'une démarche participative, l'adhésion des populations permettant de les légitimer. Toutefois, il faut alors éviter l'instrumentalisation de la «participation » comme méthode de gestion des projets, 
car elle peut renforcer les effets de sélection et l'identification des groupes cibles peut reposer sur des représentations a priori de la société rurale.

\section{Des innovations pour réduire la pauvreté rurale ?}

Existe-t-il des innovations, techniques mais également organisationnelles ou institutionnelles, qui peuvent permettre de combattre la pauvreté et la vulnérabilité des ménages ruraux, étant entendu qu'ils ne se confondent pas forcément avec des unités d'agriculture familiale et qu'ils peuvent être même déconnectés de l'activité agricole?

Si on se pose ainsi la question des innovations réduisant la pauvreté en zone rurale, c'est-à-dire en dehors des innovations techniques ou organisationnelles qui affectent le processus de production dans l'agriculture, on peut retenir essentiellement des innovations organisationnelles ou institutionnelles permettant d'améliorer l'accès à l'alimentation et aux différents éléments du bien-être. La question centrale est celle des acteurs qui portent l'innovation et qui ne peuvent plus être uniquement les producteurs agricoles ou l'ensemble des institutions, publiques ou privées, qui interviennent dans les processus d'innovation technologique en agriculture.

En fait, on peut considérer d'abord que ces innovations, qui visent par exemple à définir de nouvelles stratégies d'accès aux revenus, sont d'abord mises en œuvre par les unités économiques titulaires de ces revenus, c'est-à-dire les ménages, à travers leurs pratiques et leur choix d'activités. Mais on peut envisager aussi des démarches d'action collective au niveau des territoires ainsi que des politiques publiques. Nous allons reprendre ces différents niveaux.

\section{Les ménages ruraux peuvent-ils développer des innovations pour réduire leur exposition à la pauvreté et la vulnérabilité ?}

La pauvreté et la vulnérabilité des ménages ruraux renvoient à leurs " modes de vie » (livelihoods) conditionnés par la détention d'un ensemble de « capitaux » (Ellis et Freeman, 2005). On distingue habituellement le capital physique ou productif, auquel on peut ajouter le capital foncier, le capital humain, le capital financier et le capital social. Ces actifs peuvent être investis dans un ensemble d'activités visant à garantir au ménage un niveau de bien-être mais surtout une capacité à résister aux chocs qui peuvent affecter leurs moyens d'existence. La notion de gestion du risque est centrale dans ces stratégies. La capacité de résilience du ménage dans la gestion de son portefeuille d'activités, qui renvoie à sa stratégie, va déterminer sa sortie ou non de la pauvreté et de la vulnérabilité. L'innovation envisagée à ce niveau renvoie donc à l'émergence de nouvelles activités et de nouvelles sources de revenus et d'actifs associés.

Peut-on considérer que des stratégies de sortie de l'agriculture des ménages ruraux, telles que la diversification des activités mais aussi la migration, sont des stratégies innovantes pour échapper à la pauvreté ? Nous pouvons prendre l'exemple de 
la migration, tant ce phénomène est devenue important pour certains territoires ruraux (Requier-Desjardins, 2010).

Il peut paraître a priori surprenant de considérer que les différentes formes de migration que l'on rencontre en zone rurale, temporaire ou permanente, nationale ou internationale, puissent être considérées comme des stratégies innovantes. Il faut cependant prendre en compte les éléments mis en évidence par la littérature sur la migration et sur l'importance des transferts de migrants dans l'économie des zones rurales. Tout d'abord, face à une littérature qui a d'abord souligné les facteurs push de la migration, vu comme une décision totalement contrainte, les analyses tant de la nouvelle économie de la migration de travail ou les travaux sociologiques sur les réseaux migratoires montrent que la migration est une décision avant tout familiale et qui vise à optimiser l'utilisation des ressources des ménages. Le rapport PNUD 2009 sur le développement humain consacré à la migration met en évidence, dans une perspective de "capabilités », que la mobilité est un droit humain et que les migrants expriment avant tout un projet de vie en migrant. Le débat sur l'utilisation des transferts de migrants dans les zones rurales des pays du Sud, quoi que très controversé, met en évidence un relatif consensus sur leur contribution à la diminution de la pauvreté, mais surtout au renforcement du capital humain des populations qui en bénéficient, ce qui est en soi un élément favorable à l'innovation. La question du financement des investissements productifs et du développement par ce biais de nouvelles activités, voire du transfert de technologie, est débattue mais certains éléments semblent prouver que les transferts peuvent être une source d'amélioration des infrastructures, de développement financier, voire d'adoption de nouvelles technologies, par exemple dans le domaine de l'irrigation. Le caractère circulatoire de la migration peut jouer un rôle important en ce sens.

La mise en avant de ces différents éléments par la littérature a pu conduire à des positions assez radicales : ainsi Rigg (2006) affirme que la lutte contre la pauvreté en zone rurale doit être, dans de nombreux territoires du Sud, déconnectée de la question du développement agricole. Clements et Pritchett (2008), en intégrant les revenus des migrants aux revenus des résidents, considèrent que la migration n'est pas une alternative au développement mais qu'elle est constitutive du développement dans les pays concernés.

Le raisonnement ne concerne pas uniquement les différentes formes de migration, mais aussi la diversification des activités en dehors de l'agriculture qui nécessite l'acquisition de nouvelles compétences de la part d'un certain nombre de membres du ménage. Des expériences nombreuses concernent par exemple le développement d'activités d'artisanat, alimentaire ou non, par exemple par des collectifs de femmes (Boucher et al., 2010).

\section{Au plan méso-économique, quelles innovations territoriales?}

Au niveau méso-économique les innovations organisationnelles vont occuper un rôle essentiel, notamment au plan de la gouvernance des territoires ruraux. Elles reposent notamment sur des processus d'action collective, par exemple sur le renforcement des infrastructures territoriales d'éducation ou de santé, ou sur la mise 


\section{Encadré 5.2. Syal et développement rural : l'Alliance des agro-industries rurales de la Selva Lacandona, Chiapas}

\section{Francois Boucher, Denis Requier-Desjardins et Virginie Brun}

Les concentrations géographiques d'agro-industries rurales (AIR), que l'on observe surtout en Amérique latine, donnent une identité territoriale forte aux dynamiques de développement local, qui reposent sur des articulations complexes entre territoire, acteurs, produits et système d'innovation (Boucher et al., 2003).

Partant de ce constat, un projet de développement de micro-entreprises rurales a été impulsé dans plusieurs communautés indiennes de la Selva Lacandona du Chiapas au Mexique, région qui compte parmi les plus isolées et les plus marginalisées et pauvres du pays. L'objectif de ce projet était triple : réduire la pauvreté dans plusieurs microrégions de la Selva Lacandona ; diminuer la pression sur les ressources naturelles et ralentir les processus de dégradation environnementale ; intégrer les politiques de développement social à un cadre de développement territorial, participatif et soutenable. Deux grandes lignes d'action ont ainsi été définies sur la base de ces objectifs : d'une part, l'aide à l'organisation et à la mise en route de 16 AIR de différents types (transformation agroalimentaire, artisanat, micro-usine de potabilisation d'eau, coopératives de vente, etc.), et d'autre part, le renforcement des capacités de gestion et d'innovation des acteurs impliqués. Différents ateliers participatifs ont ainsi été organisés autour de thèmes identifiés comme prioritaires : innovation technologique, organisation et gestion d'une entreprise, amélioration des procédés de production, analyse de marché et commercialisation. Cette première étape a ensuite permis d'induire une dynamique territoriale collective autour de la consolidation de ces différentes AIR, de la diffusion des savoir-faire et de l'innovation, dynamique qui s'est concrétisée par la formalisation d'une organisation, «l'Alliance des AIR de la Selva Lacandona », et la création d'une marque collective «Agro-industries rurales de la Selva Lacandona ».

Une des principales leçons tirées de ce projet de développement a été de pouvoir identifier et définir les conditions de viabilité des AIR de la Selva Lacandona. Si la rentabilité économique des micro-entreprises s'avère essentielle pour garantir leur viabilité, celle-ci ne semble pas centrale dans le sens où elle ne pose pas de réels problèmes. En revanche, deux aspects sont apparus comme fondamentaux pour assurer le développement durable d'AIR dans une région marginalisée comme celle de la Selva Lacandona : la nécessité d'un environnement préalable favorable, et la résolution des problèmes d'organisation et de leadership. Le premier point, déjà soulevé par de Janvry et al. (2002) dans leur approche en trois phases de la réduction de la pauvreté, se réfère à la nécessité d'investir en premier lieu dans les conditions préalables (éducation, santé, nutrition, infrastructures et services de base) indispensables à la création et à la pérennisation de petites entreprises en milieu rural. Il est en effet apparu qu'une dynamique de type Syal, basée sur une diffusion horizontale des processus d'innovation et sur le savoir-faire et les ressources spécifiques d'un territoire, est intrinsèquement liée à la présence et à l'usage de biens publics locaux fonctionnels, ce qui fait en général défaut dans les régions marginalisées. Le second point se réfère aux problèmes d'organisation, d'action collective et de leadership au sein des groupes, qui résultent le plus souvent d'une méfiance entre les membres et d'une définition des rôles mal comprise. La principale conséquence est un blocage des dynamiques collectives, qui se manifeste par la présence de " passagers clandestins » au sein des groupes et par des situations dans lesquelles les leaders se comportent comme « le chien du jardinier », autrement dit qu'ils ne veulent ni faire ni laisser-faire (Boucher et al., 2010). 
en place de stratégies de commercialisation permettant d'améliorer le revenu des producteurs. Dans cette catégorie, on peut notamment citer :

- les stratégies liées à la mise en place de dispositifs de commerce équitable comme l'ont illustré par exemple les démarches mises en œuvre autour de la commercialisation du café équitable et qui implique la formation de collectifs de producteurs et l'investissement d'une partie des revenus dans la réalisation d'infrastructures communautaires ;

- les stratégies innovantes de commercialisation, qui peuvent notamment recouvrir le développement des filières courtes et l'établissement de liens de proximité avec les consommateurs. Elles tendent vers une territorialisation de la production agricole et une mutualisation des risques productifs avec les consommateurs. Si ce type d'expérience a surtout été développé au nord (AMAP par exemple en France) quelques expériences ont vu le jour au sud, surtout en Amérique latine, portant sur la création de marchés de producteurs, de marchés bios ou de filières courtes approvisionnant les villes (Sao Paulo).

Dans certains contextes, comme par exemple celui des Andes étudiés par Fernandez-Baca et al. (2010), on constate que les projets d'innovation des communautés reflètent la diversification des activités et des structures d'actifs. L'importance des projets portant sur l'artisanat et le tourisme dans les communautés rurales des pays du Sud doit également être soulignée.

L'innovation au niveau du territoire peut être appréhendée comme le positionnement autour de ses « actifs spécifiques » et la mise en place d'une stratégie de qualification des territoires.

De ce point de vue, les stratégies innovantes de qualification des territoires ne concernent pas simplement les produits agricoles. L'approche en termes de «panier de biens » (Pecqueur, 2001) permet de mettre en évidence une articulation entre différentes activités et différents biens et services, privés ou publics, qui partagent une caractéristique ${ }^{7}$ de qualification territoriale. Cette caractéristique est construite notamment par une interaction avec les consommateurs qui la valident, et elle peut être renforcée par des dispositifs institutionnels qui ressortent de l'action collective. Notamment dans le cadre de la diversification des activités en zone rurale, elle peut favoriser au plan du territoire une synergie de l'agriculture et des autres activités, par exemple à travers l'existence de « systèmes agroalimentaires localisés » centrés sur les produits de qualité. La durabilité d'une telle trajectoire de développement territorial suppose cependant une certaine stabilisation de la demande s'adressant à ce panier de biens. Or cette demande est largement nourrie par les différentes formes de base résidentielle et présentielle, autour des aménités du territoire. Il suffit de penser par exemple au rôle du tourisme, mais celui des diasporas de migrants peut être tout aussi important. On peut donc se poser la question de la réplication de ce type de trajectoire. Quelle portée sur les territoires ruraux de quels pays ? La prise en compte des innovations au niveau territorial, plutôt qu'à celui des ménages, permet de s'interroger sur la dimension des politiques et des projets qui peuvent être définis à ce niveau.

7. Au sens de Lancaster (1971). 
Encadré 5.3. Enseignements tirés du « Panorama Andino » sur l'innovation rurale dans les Andes

Edith Fernandez-Baca, Maria Paz Montoya et Natalia Yañez.

Le Consortium pour le développement durable de l'écorégion andine (CONDESAN - Consortium for the Development of the Andes Ecoregion) a conduit une étude pour répertorier les expériences d'innovation rurale dans la région concernée. Vingt exemples ont été analysés dans les zones rurales andines de sept pays (Argentine, Bolivie, Chili, Colombie, Équateur, Pérou et Venezuela).

Cette étude a montré que ces pays, confrontés à une grande diversité de problèmes de développement et de croissance économique, utilisent différents types d'instruments pour faire face aux réalités économiques, sociales et environnementales qui leur sont propres. Les politiques de développement, de croissance et de lutte contre la pauvreté varient également d'un pays à l'autre.

D'un point de vue interne, une telle hétérogénéité peut être considérée comme une force puisqu'elle découle des capacités et des compétences locales et/ou nationales et du degré de développement institutionnel de chaque segment de la société. Cependant, elle est également de nature à faire obstacle à la mise en place de programmes communs en matière d'innovation dans la région andine. Il reste toutefois possible de tenter de comprendre comment la capacité à innover se développe en suivant des scénarios différents dans un contexte de rareté et de pauvreté. Ces enseignements peuvent alors être transposés en pratiques publiquement reconnues à l'échelle locale et régionale, puis adaptés de manière créative en stimulant de nouveaux processus d'innovation endogènes.

En matière d'innovation, il existe des opportunités et des solutions. Les opportunités d'innover sont économiques, sociales, environnementales et institutionnelles, tandis que les solutions (ou les innovations qui permettent de faire se rejoindre des besoins et des opportunités) peuvent être liées à des techniques afférentes à la production et aux services, relever de l'organisation ou de la commercialisation, de l'aménagement du territoire, ou encore se présenter comme une combinaison de ces différents aspects.

Dans la pratique, plutôt que des systèmes d'innovation ruraux, on observe le renforcement des réseaux de coopération - principalement sous l'impulsion des notables locaux - visant à assurer l'apprentissage collectif et l'accumulation d'expérience pratique par DUI (Doing, Using, Interacting, ou « faire, utiliser, interagir »). Il manque cependant à ces réseaux un acteur qui ferait le lien, d'une part entre les organismes de recherche-développement et les acteurs de la société civile qui génèrent le savoir et les innovations, et d'autre part, les organisations rurales sociales et productives qui ont besoin de savoirs et d'innovations. Ce problème devient un défi que les politiques locales et nationales se doivent de relever.

Il est encore aujourd'hui nécessaire de renforcer les politiques ciblant la réduction de la pauvreté, qui peuvent ne pas être à proprement parler des politiques d'innovation mais qui sont susceptibles de servir de base pour combattre la pauvreté dans toutes ses dimensions. Leur rôle clé dans le renforcement du capital humain et des capacités des plus démunis permet de constituer un environnement propice à l'innovation (Fernandez-Baca et al., 2010). 


\section{Quelles innovations pour les politiques publiques au niveau national?}

Les politiques publiques qui s'attaquent à la pauvreté, et plus particulièrement à la pauvreté rurale, empruntent deux voies :

- d'abord, elles reposent sur l'orientation pro-poor de l'ensemble des politiques économiques développées en direction de l'agriculture et des zones rurales, en essayant de maximiser les externalités positives de ces politiques. La question de la mesure de l'impact sur la pauvreté et en particulier sur l'extrême pauvreté reste posée, du fait de la difficulté du ciblage, qui peut se traduire par des effets de sélection entre les pauvres, comme nous l'avons souligné pour les politiques d'innovation agricole et agroalimentaire ;

- puis, on a vu apparaître récemment des politiques de lutte directe contre la pauvreté, essentiellement sous la forme de transferts sous conditions. Des programmes innovants ont cherché à améliorer le ciblage de ces politiques en direction des plus pauvres et des plus vulnérables. Ainsi des politiques macro-sociales de lutte contre la pauvreté en zone rurale (opportunidades au Mexique, bolsa familia au Brésil), créant avant tout une conditionnalité sur le renforcement du capital humain des bénéficiaires et ciblant par exemple les femmes au sein des ménages ainsi que la scolarisation des enfants, ont représenté des innovations importantes en matière de lutte contre la pauvreté dans des zones rurales ${ }^{8}$, mais elles sont en soi déconnectées de toute référence spécifique à l'agriculture, ce qui nous renvoie à la problématique de la relation entre agriculture et pauvreté rurale.

Les politiques publiques d'appui à l'innovation en agroalimentaire, quant à elles, mobilisent les acteurs classiques de l'innovation, notamment ceux de la recherche-développement et de la vulgarisation et développent des systèmes d'incitation vis-à-vis des acteurs privés.

Il faut souligner l'importance de la contextualisation tant des politiques de lutte contre la pauvreté rurale que des politiques d'innovation agricole et agroalimentaire au sens strict. Cette contextualisation a souvent un caractère local ou régional et renvoie également à la spécialisation agricole. Par exemple, dans certains territoires où l'agriculture représente la source de revenu essentiel des ménages ruraux, il est possible qu'une politique d'intensification agricole ait des effets positifs sur la pauvreté. Dans d'autres au contraire, où les sources de revenus sont beaucoup plus diversifiées, la ruralité apparaît comme une entrée privilégiée pour penser une politique de développement territorial. Les structures agraires, l'importance relative des agricultures familiale et entrepreneuriale notamment, de même que l'orientation technico-économique des systèmes de production, sont évidemment un élément déterminant de ce choix.

8. On considère que Bolsa Familia au Brésil est responsable de la baisse significative du coefficient de Gini enregistrée depuis le début des années 2000. 


\section{$\rightarrow$ Conclusion}

Au terme de ce chapitre, il convient de s'interroger sur la question suivante : quels acteurs et quelles politiques publiques pour quels types d'innovation? Il nous paraît pertinent de partir des innovations pour pouvoir ensuite aborder la question des acteurs et des politiques.

L'éventail des innovations que nous avons été amenés à couvrir est particulièrement vaste puisqu'il couvre les innovations agricoles autour de l'intensification, qu'elle soit « productiviste » ou " agro-écologique », les innovations institutionnelles ou organisationnelles menées tant au plan du secteur agroalimentaire au sens large que des territoires ruraux. La dualité secteur/territoire dans le champ de l'innovation nous est apparue particulièrement importante. Or chaque type d'innovation suppose la définition de "parties prenantes », ayant un enjeu autour du processus et des porteurs de l'innovation (recherche, pouvoirs publics, entreprises, etc.). $\mathrm{Si}$ les porteurs de l'innovation peuvent être généralement bien identifiés, la question des parties prenantes est beaucoup plus délicate, dans la mesure où on peut poser comme principe que l'inscription de l'innovation dans un processus de développement durable doit reposer sur son caractère participatif, nécessitant un compromis entre les parties prenantes, la recherche agronomique, les producteurs, les organisations paysannes mais aussi la société civile et l'ensemble des acteurs des systèmes de gouvernance. En revanche, le succès de l'innovation exige que le processus soit maîtrisable au plan de l'action collective des acteurs qui la portent.

Si l'enjeu est celui de l'innovation technologique en agriculture ou en agro-alimentaire, la mise en œuvre de l'innovation implique des effets de sélection entre acteurs, notamment dans le processus de diffusion. Ces effets de sélection sont probablement une condition du succès de l'innovation. Si l'enjeu est celui de l'impact des politiques d'innovation sur la pauvreté rurale, il faut probablement retenir un processus participatif qui permette d'inclure les «plus pauvres des pauvres » et justement éviter les effets de sélection. On est donc confronté à un dilemme renforcé encore par le décalage entre la dimension territoriale du développement rural et de la réduction de la pauvreté rurale, et la dimension sectorielle des innovations technologiques ou organisationnelles en agroalimentaire.

Les politiques publiques concernées se déclinent donc en trois rubriques :

- des politiques ciblées sur la réduction de la pauvreté qui ne sont pas en soi des politiques d'innovation, mais qui peuvent constituer un socle dans la lutte contre la pauvreté dans ses différentes dimensions. Leur conditionnalité dans le renforcement du capital humain et des capacités des plus pauvres constituent un environnement favorable à l'accueil de l'innovation ;

- des politiques d'innovation dans le secteur agroalimentaire qui, dans une exigence de développement durable, doivent se tourner vers la recherche de l'intensification agro-écologique : ces politiques sont plus enclines que les politiques d'innovation intensificatrice traditionnelle à avoir une dimension territoriale affirmée et à cibler les acteurs de l'agriculture familiale; le résultat peut être favorable à la réduction de la pauvreté rurale, mais ce n'est pas forcément leur objectif initial ;

- des politiques territoriales de développement rural : c'est à ce niveau que peut s'organiser la mise en cohérence de la lutte contre la pauvreté rurale et de 
l'innovation pour un développement durable mais ces politiques doivent introduire une dimension importante d'innovation institutionnelle.

Le champ des politiques publiques concernées excède donc largement l'habituelle définition des politiques de développement agricole et les frontières habituellement définies de la ruralité. La difficulté est celle d'une approche globale et compréhensive de ces politiques du fait de la segmentation des compétences et des institutions chargées de les appliquer. C'est donc dans la capacité de les articuler que réside l'enjeu essentiel de leur contribution à un développement rural durable qui intègre la dimension sociale de la durabilité.

\section{" Références bibliographiques}

Boucher F., Requier-Desjardins D., Brun V., 2010. SYAL : un nouvel outil pour le developpement de territoires marginaux. Les leçons de l'alliance des agro-industries rurales de la Selva Lacandona, Chiapas, In : Innovation and Sustainable Development in Agriculture and Food (ISDA), Montpellier, France -http://hal.archives-ouvertes.fr/hal-00521013/fr/

Clasadonte L., 2008. Network companies, another way on thinking agriculture. A supply chain management vision in South America, Master thesis, Wageningen University.

Clemens M., Pritchett L., 2008. Income per natural: measuring development as if people mattered more than places, Center for Global Development, $\mathrm{n}^{\circ} 143$, mars 20.

Conway G., 1997. The doubly green revolution: food for all in the 21st century, Penguin, London.

Davezies L., 2008. La France et ses territoires, la circulation invisible des richesses, Seuil, Paris.

De Janvry A., Aranjo C., Sadoulet E., 2002. El desarrollo rural con una visión territorial, Universidad de California en Berkeley, $12 \mathrm{p}$.

De Grammont H., 2010. México: boom Agricola y persistencia de la pobreza rural, In: Boom Agrícola y persistencia de la pobreza rural, CEPAL, 225-261.

Dethier J.-J., Effenberger A., 2011. Agriculture and development: a brief review of the literature, Policy research Working Paper 5553, Banque mondiale.

Dietsch R., Ruault C., 2010. Dispositifs d'appui à des processus locaux d'innovation et integration des paysans pauvres dans les montagnes sèches d'Amérique Centrale : une difficile articulation, In : Innovation and Sustainable Development in Agriculture and Food (ISDA), Montpellier, France - http://hal.archives-ouvertes.fr/hal-00522726

Dubois J.L., Mahieu F.R., 2002. La dimension sociale du développement durable: réduction de la pauvreté ou durabilité sociale ? In : Développement durable ? Doctrines, pratiques, évaluations (Martin J.Y., ed.), IRD, Paris, 73-94.

Ellis F., Freeman H., 2005. Rural livelihoods and poverty reduction policies, Routledge, Londres, $405 \mathrm{p}$.

Fernandez-Baca E., Montoya M.P., Yañez N., 2010. Innovation for poverty reduction with inclusion in the andean region. Panorama Andino - Learning from case studies on locally promoted innovation experiences, In : Innovation and Sustainable Development in Agriculture and Food (ISDA), Montpellier, France -http://hal.archives-ouvertes.fr/hal-00523493/en/

Gereffi G., 1999. International trade and industrial upgrading in the apparel commodity chain. Journal of International Economics, 48, 37-70.

Gereffi G., Humphrey J., 2005. The governance of global value chains. Review of International Political Economy, 12-1, 78-104.

Giuliani E., 2007. The selective nature of knowledge networks in clusters: evidence from the wine industry. Journal of Economic Geography, 7, 139-168.

Griffon M., 2006. Nourrir la planète : pour une révolution doublement verte, Odile Jacob, Paris.

Haggblade S., Hazell P., Reardon T., 2010. The Rural Non-Farm Economy, prospecs for growth and poverty reduction. World Development, 38-10, 1414-1441. 
Hernandez V., 2008. El fenómeno económico del boom de la soja y el empresariado innovador. Desarrollo económico, 47-187, 331-365.

Kline S., Rosenberg N., 1986. An overview of innovation, In : The positive sum strategy (Landau R., Rosenberg N., eds), National Academy Press, Washington.

Lancaster K., 1966. A new approach to consumer's theory. Journal of Political Economy, 74 (2), 132.

Lipton M., 2006. Can small farmers survive, prosper, or be the key channel to cut mass poverty? The Electronic Journal of Agricultural and Development Economics, 3 (1), 58-85.

Nelson R., Winter S., 1982. For an evolutionary theory of economic change, Harvard University Press, Cambridge.

North D., 1990. Institutions, institutional change and economic performance, Cambridge University Press.

Paillard S., Treyer S., Dorin B., 2010. Agrimonde : scénarios et défis pour nourrir le monde en 2050, éditions Quae, Versailles.

Parrot L., Pedelahore P., De Bon H., Kahane R., 2010. Urban and peri-urban horticulture and the capability approach: the case of the south-west province of cameroon, In : Innovation and Sustainable Development in Agriculture and Food (ISDA), Montpellier, France - http://hal.archivesouvertes.fr/hal-00516466/fr/

Pecqueur B., 2001. Qualité et développement rural : l'hypothèse du panier de biens et services territorialisés. Economie rurale, 261, 37-49.

Porter M., 1990. The Competitive Advantage of Nations, Free Press, New York.

Rawls J., 1971. Théorie de la justice, traduction française, 1987, Seuil.

Reardon T., Timmer C.P., 2007. Transformation of markets for agricultural output in developing countries since 1950. How has thinking changed? In : Handbook of Agricultural Economics (Evenson R.E., Pingali P., eds.), Vol. 3, Agricultural Development: Farmers, Farm Production and Farm Markets. Elsevier Press, Amsterdam, 2808-2855.

Reardon T., Barret C., Berdegué J., 2009. Agrifood industry transformation and small farmers in developing countries. World Development, 37-11, 1717-1727.

Requier-Desjardins D., 2010. International migration from Southern countries rural areas: which impact on agricultural and rural sustainability? In : Innovation and Sustainable Development in Agriculture and Food (ISDA), Montpellier, France - http://hal.archives-ouvertes.fr/hal-00521365

Rigg J., 2005. Land farming, livelihoods and poverty: rethinking the links in the rural south. World Development, 34-1, 180-202.

Schumpeter J., 1911. Théorie de l'évolution économique traduction française, 1935, Paris, Librairie Dalloz.

Sen A., 1982. Poverty and Famines: An Essay on Entitlements and Deprivation, Clarendon Press, Oxford.

Sen A., 1987. On ethics and economics, Basil Blackwell, Oxford.

Sen A., 1999. Development as freedom, Oxford University Press, Oxford. 



\title{
Chapitre 6
}

\section{Innovations de marché axées sur la qualité : dimensions sociales et d'équité}

\author{
Estelle Biénabe, Cerkia Bramley, Johann Kirsten
}

Ce que nous appelons innovations de marché dans les systèmes agroalimentaires correspond essentiellement - en particulier d'après les contributions présentées à l'occasion du symposium ISDA - à des innovations organisationnelles ou institutionnelles qui concernent plusieurs acteurs liés entre eux par leurs interventions, directes ou indirectes, au sein des filières (à savoir, les producteurs, les transformateurs de l'agrobusiness, les revendeurs, les consommateurs, les ONG et les organismes publics). Ces liens reflètent des formes de plus en plus sophistiquées d'intermédiation et de coordination à l'intérieur des filières (mécanismes de contrat et schémas d'approvisionnement, certification, stratégies d'étiquetage et d'image de marque, etc.) qui, dans bien des cas, représentent des innovations majeures ayant des répercussions significatives en matière de durabilité. Ces innovations, qui concernent un ensemble de plus en plus diversifié de parties prenantes, peuvent être liées à plusieurs types de préoccupations en matière de développement durable, et plus particulièrement à des problèmes écologiques (tels que l'exploitation et la dégradation des ressources) ou de déséquilibre des relations commerciales.

Nous nous intéresserons ici plus spécifiquement au rapport qui existe entre les innovations de marché axées sur la qualité et l'accès au marché des petits agriculteurs, à savoir les conditions et le niveau de leur participation à la chaîne d'approvisionnement alimentaire. Il est important de noter que le problème de l'équité dans l'accès au marché n'est en rien statique et qu'il est, au contraire, extrêmement dynamique. Ainsi, selon Berdegué et al. (2011), « assurer une inclusion pérenne est beaucoup plus difficile et insaisissable que d'obtenir un accès initial à des marchés dynamiques ». Nous explorerons les conséquences, pour le développement durable, de plusieurs innovations de marché axées sur la qualité, en nous inspirant, d'une part 
des réflexions provenant de la littérature sur le sujet et d'autre part, d'études de cas mettant en évidence la diversité des éléments à prendre en considération et des vecteurs de changement dans les filières.

\section{" Innovations axées sur la qualité et implications pour les petits agriculteurs}

\section{Une approche globale de la filière}

L'approche analytique adoptée dans ce chapitre vise à analyser et à évaluer les innovations de marché et leurs répercussions en considérant la filière dans son ensemble. Elle fait appel aux outils analytiques mis au point dans le contexte des chaînes de valeur globales. Ainsi qu'il a été souligné dans l'introduction, les innovations de marché axées sur la qualité sont principalement de nature organisationnelle ou institutionnelle et s'appuient sur les rapports qu'entretiennent les divers acteurs qui interviennent directement ou indirectement dans la filière. L'approche qui consiste à appréhender cette dernière dans sa globalité s'avère particulièrement utile pour comprendre les conséquences sociales des innovations de marché. En effet, le cadre conceptuel qu'elle apporte permet de mieux analyser les aspects de la gouvernance au sein des filières et la capacité des divers acteurs à tirer profit de leur participation et à la pérenniser. La gouvernance d'une filière dépend de l'influence qu'exerce chaque partie prenante sur cette filière, et de la capacité des divers acteurs à dicter les règles qui s'y appliquent (c'est-à-dire qui régissent les rapports entre acteurs). La concentration qui s'est opérée au niveau de la transformation et de la distribution a favorisé la position dominante d'un petit nombre d'entreprises agroalimentaires dans le contrôle de nombreuses filières de ce secteur.

La gouvernance des filières est par ailleurs encadrée par des dynamiques de régulation plus générales, et notamment par les interactions entre les régulations publiques, ou émanant de l'État, et les activités de régulation du secteur privé. Les manifestations de ces dynamiques sont parfois propres à certaines filières. Ainsi qu'il a été relevé dans les discussions qui ont eu lieu pendant le symposium ISDA, prendre en considération les problèmes de gouvernance et les interactions entre les régulations publiques et les actions des parties prenantes privées contribue à combler la division conceptuelle - trop souvent supposée - entre le marché et l'État.

\section{Innovations axées sur la qualité et sophistication de la coordination}

Ainsi qu'il a été mis en évidence au cours du symposium ISDA, les innovations de marché axées sur la qualité sont principalement associées à la mise au point de normes. Il est désormais communément admis que la mise en place progressive de chaînes d'approvisionnement axées sur la qualité et fonctionnant par le biais de normes modifie de manière significative les modes de coordination au sein des 
filières concernées. En remodelant l'organisation de la production et les relations commerciales, elle agit sur la gouvernance et sur la participation aux marchés. De nombreux auteurs ont en effet attiré l'attention sur l'abandon progressif, dans les filières longues et dans les filières axées sur la qualité, des marchés spots caractérisés par l'anonymat des fournisseurs et par l'absence de responsabilité clairement identifiée, vers plus de formes de coordination verticale (voir notamment Gibbon et Ponte, 2005). Selon Ruben et al. (2006), cette plus grande coordination verticale, qui s'appuie sur des dispositions contractuelles complexes, vient de pair avec une surveillance plus étroite de la qualité des produits et des normes portant sur les procédés. La concurrence entre acteurs individuels tend à s'estomper au profit d'une concurrence entre filières (Hanf et Pienadz, 2007). Une coordination renforcée entre les acteurs tout au long de la filière est déterminante pour satisfaire la fourniture de nouvelles dimensions de la qualité et générer ainsi de la différenciation. Ceci va dans le même sens que les observations empiriques et l'analyse de Wilson et al. (2000) concernant les performances commerciales de deux produits d'appellation d'origine protégée (des pommes de terre précoces du Royaume-Uni et des Pays-Bas), qui montrent que des disparités dans les niveaux de coopération et de coordination au sein de la filière se traduisent par des différences significatives en ce qui concerne les caractéristiques techniques du produit, la promotion de la marque et le degré de sensibilisation des consommateurs.

Toutefois, bien qu'une coordination verticale renforcée et des systèmes de gestion plus sophistiqués de la qualité soient utilisés dans le cadre de stratégies de différenciation par des acteurs dominants des filières, il convient de souligner qu'en parallèle l'action collective se développe entre de grandes filières et entre entreprises, en particulier au niveau de la grande distribution. Le référentiel GlobalG.A.P. illustre de manière particulièrement parlante les efforts des distributeurs pour standardiser les filières internationales (Henson et Reardon, 2005). Le passage de normes propres à un distributeur particulier à des normes s'appliquant à un groupe de distributeurs - elles-mêmes contrôlées par la certification par tierce partie - entraîne un resserrement de la coordination horizontale lorsque ces normes privées, collectives et volontaires sont adoptées (Havinga, 2008). Il existe en effet des intérêts communs à limiter les coûts de transaction correspondant à la mise en place de ces normes. Fulponi (2006a) a analysé l'émergence de coalitions d'entreprises en vue d'instaurer des normes privées, collectives et volontaires comme un élément clé de l'évolution des systèmes alimentaires. GlobalG.A.P., mais également la certification Eco-Friendly, les directives de l'IFOAM pour l'agriculture biologique, et les initiatives Fairtrade et Ethical Trading en sont plusieurs exemples. Le fait que ces normes entraînent une coordination à la fois verticale et horizontale est confirmé par Hammoudi et al. (2009).

Les études qui se fondent sur l'économie des coûts de transaction aident à mieux comprendre cette évolution vers une plus grande coordination dans les filières et une plus grande complexité des systèmes de gestion de la qualité. Raynaud et al. (2002) analysent la structure de la gouvernance dans 42 études de cas réalisées dans trois secteurs agroalimentaires et dans sept pays européens. Ils montrent que l'agent qui met au point un signal de qualité dont la valeur dépend d'autres acteurs de la filière élabore des mécanismes spécifiques pour régir les transactions à un niveau bilatéral afin de s'assurer une crédibilité par un système fiable de garantie du produit. Il peut 
en découler divers types de rapports contractuels en fonction des signaux de qualité. Barcala et al. (2007) insistent également sur le fait que l'impact sur la qualité des produits diffère selon les mécanismes de gouvernance et les formes d'organisation. Leurs résultats indiquent que les mécanismes de gouvernance les plus axés sur le marché relevés dans leur étude (quasi intégrations et indications géographiques) vont de pair avec :

- des mécanismes de coordination tels que des règles et des routines visant à définir parfaitement les normes et les spécificités ;

- un ensemble complémentaire de dispositifs de contrôle de la qualité fondés sur une supervision directe.

Dans la même veine, Ménard et Valceschini (2005) font remarquer que le type de signal de qualité influence la gouvernance des transactions, ajoutant que les marques privées sont plus souvent associées à une intégration verticale que les marques qui ont un lien avec une certification publique ${ }^{1}$. Ils insistent en outre sur l'importance de prendre en considération le lien entre, d'une part, les structures de gouvernance au niveau des filières conçues pour régler des problèmes particuliers de signalisation de la qualité et d'autre part, le niveau macroéconomique auquel les mécanismes institutionnels sont mis au point afin de renforcer la crédibilité des modes d'organisation adoptés (tels que le recours croissant à la certification par tierce partie accréditée par des institutions publiques).

Ponte et Gibbon (2005) font valoir que la capacité à capturer des informations complexes concernant la qualité dans les normes, les labels, les certifications et les codifications atténuent le besoin d'intégration verticale engendré par l'amélioration de la signalisation de la qualité et par la complexification de la gestion. En effet, dans de nombreuses filières de produits à haute valeur ajoutée, les entreprises dominantes utilisent des normes et des stratégies d'image de marque pour exercer un contrôle sur les fournisseurs sans nécessairement passer par la structure de propriété (CNUCED, 2007). Selon Ponte et Gibbon (2005), le contrôle du mode de qualification et de la gestion de l'information joue un rôle important dans l'aptitude des entreprises dominantes à exercer leur « leadership fonctionnel». Ces auteurs ont montré que la capacité des entreprises à transférer des informations relativement immatérielles à leurs fournisseurs et à standardiser, et/ou à faire certifier à l'extérieur, les multiples dimensions de plus en plus complexes de la qualité des biens et des services autorise des formes de coordination relativement flexibles et un dynamisme important. La CNUCED (2007) souligne également l'importance accrue, pour faire face à la concurrence et maîtriser les filières, de s'assurer le contrôle et la possession des éléments d'actifs incorporels - et notamment les informations et les marques - plutôt que des moyens physiques de production.

1. La certification est une procédure par laquelle une tierce partie donne son assurance écrite qu'un produit, un procédé ou un service est en conformité avec certaines normes (Guide ISO, 1996). On appelle organisme de certification ou certificateur l'organisation chargée de la certification. Il peut conduire l'audit ou l'inspection lui-même, ou sous-traiter cette opération à un organisme d'audit ou d'inspection. Le système des règles, des procédures et de gestion utilisées pour la certification, et qui comprend le référentiel au regard duquel la société est certifiée, est appelé programme de certification. Un organisme de certification peut conduire divers programmes de certification. La capacité de ces organismes de certification à se charger d'un programme de certification est évaluée et accréditée par une institution ayant autorité (FAO, 2007). 


\section{Petits agriculteurs et différents types de dynamiques de qualité dans les filières}

Reardon et al. (2003) signalent les effets de la sophistication des filières en termes de renforcement du pouvoir de négociation des acteurs de l'aval et d'exclusion des petits producteurs agricoles, notamment dans le contexte des pays en voie de développement. Codron et al. (2005) abordent ces questions du point de vue des stratégies de qualité adoptées par les distributeurs lorsqu'ils sont confrontés à un système de gestion de la qualité qui leur est imposé. Ils soulignent que l'allocation des coûts associés à la mise en œuvre de ces stratégies est déterminée par les rapports verticaux qui existent entre distributeurs et fournisseurs, et donc par la répartition du pouvoir de négociation entre ces catégories d'acteurs. Vorley (2001) analyse ce problème dans le contexte de la mise au point des normes et autres exigences en lien avec des considérations de durabilité apparues suite aux pressions de la société civile (ONG). À l'instar de Ponte et Gibbon (2005), il soutient que ces normes contribuent à consolider l'emprise des principaux acteurs aval sur la chaîne d'approvisionnement et à renforcer les obstacles à l'entrée sur le marché. En effet, les acteurs de l'aval ont ainsi la capacité de transférer à leurs fournisseurs la charge que représentent les coûts et les risques associés à la mise en conformité - ce qui leur permet d'intervenir de manière active dans les décisions qui sont prises à l'échelle des exploitations agricoles sans passer par une intégration verticale. Un exemple parlant de ce type d'intervention est le référentiel GlobalG.A.P. qui exige l'application de « bonnes pratiques agricoles » (Good Agricultural Practices, GAPs) au niveau de la production primaire et qui étend les principes d'identification et de gestion des risques à la production sur les exploitations. L'affaiblissement du pouvoir de négociation des agriculteurs est particulièrement inquiétant à la lumière de la diminution de l'intervention publique en soutien à l'agriculture et dans les marchés.

Le développement des filières coordonnées verticalement, caractérisées par l'utilisation de normes privées et contrôlées par des acteurs majeurs du secteur agroalimentaire, a changé les règles de participation dans les marchés. Il en a résulté une plus grande disjonction entre les prix aux producteurs et les prix sur les marchés finaux (Vorley, 2001). Dans de nombreuses régions du monde, cette évolution contribue de manière significative à confiner les petits agriculteurs sur les marchés locaux peu rémunérateurs (Ssemwanga, 2005). Elle les tient à l'écart des filières de produits à haute valeur ajoutée (dont les marchés à l'exportation) et les empêche de tirer profit des tendances en cours en matière de qualité. Cependant, Vorley (2001) met par ailleurs en avant les processus de différenciation rurale et de diversification qui interviennent actuellement dans l'économie des petites exploitations, et souligne la nécessité d'en comprendre les implications. Dans le contexte quasi général d'un environnement libéralisé, l'accès aux marchés dépend avant tout de la capacité à tirer profit des « avantages commerciaux ». Vorley (2001) insiste de ce fait sur l'importance de mieux comprendre les changements précis qui sont apparus dans les termes de l'échange liant les agriculteurs et les acteurs aval des filières en fonction des situations. Il s'agit d'identifier la nature du relèvement qualitatif observé dans les diverses filières, de la mettre en relation avec la capacité d'innovation des acteurs et d'en évaluer les répercussions. Comme le relève Giovannucci (2003), les attributs sociaux et environnementaux sont de moins en moins des facteurs de différenciation. Ils se 
rapprochent des critères de marché conventionnels et deviendront de plus en plus des prérequis indispensables pour l'intégration au sein des marchés plus développés - affectant ainsi plus fortement les petits agriculteurs. Il est de ce fait absolument crucial de bien comprendre les capacités et les limites des innovations de marché et celles des petits agriculteurs en ce qui concerne le développement et la mise à profit de leurs « avantages commerciaux ». Dans la section qui suit, nous tenterons d'apporter des éléments de réponses à ces questions en nous appuyant à la fois sur la littérature et sur les débats qui ont eu lieu à l'occasion du symposium ISDA au sujet d'un certain nombre d'innovations de marché étudiées dans différents pays.

\section{- Les effets des démarches de qualité pour les petits agriculteurs : des observations peu concluantes}

\section{Aperçu général sur les effets excluants des démarches de qualité}

Comme nous l'avons vu dans la section précédente, les filières axées sur la qualité et l'utilisation croissante de normes privées ont clairement des répercussions sur la participation des petits agriculteurs. Les normes privées sont rapidement en train de devenir le facteur clé qui détermine l'accès aux marchés (Henson et Reardon, 2005). Le respect des normes requiert des capacités suffisantes en matière d'accès aux informations pertinentes, d'adaptation des pratiques de production et de mise en application de nouveaux procédés (Giovanucci et Reardon, 2000). Qui plus est, Chemnitz (2007) montre que les normes privées sont généralement plus coûteuses que les exigences gouvernementales pour ce qui est de la communication et de la documentation des processus de certification et qu'elles sont de ce fait susceptibles d'avoir des effets excluants plus significatifs. Ces derniers sont exacerbés par le fait que les normes des distributeurs privés en particulier concentrent leur action sur les processus de gestion, en sus des contrôles de produit classiques, pour obtenir les résultats souhaités (Fulponi, 2006a). Le manque d'harmonisation entre les divers référentiels publics et privés vient encore compliquer les exigences de conformité et rend de plus en plus difficile la participation des producteurs dans ces filières (voir par exemple Henson et Humphrey, 2009).

Selon Farina et al. (2005), en termes d'investissement et de gestion, certains indices montrent que les petits agriculteurs seront plus gravement affectés par ce processus, en particulier dans les pays en voie de développement. L'absence de possibilité de bénéficier à titre individuel d'économies d'échelle contraint fortement leur capacité à se conformer au cadre normatif de plus en plus draconien et à payer les coûts croissants de mise en conformité (Dolan et Humphrey, 2000). Cette situation amplifie encore les inégalités déjà marquées qui existent entre exploitations agricoles de taille différente en ce qui concerne leur capacité à approvisionner les entreprises dominantes, qui exigent des niveaux de production élevés et qui préfèrent bien souvent collaborer avec les gros exploitants, étant donné les surcoûts de communication et de contrôle qu'il y a à travailler avec des petits producteurs (Swinnen, 
2005). Vorley (2001) se penche par ailleurs sur les possibilités de ces derniers à tirer bénéfice d'un " avantage commercial » dans un contexte où le marché favorise la capacité des gros exploitants à se charger des processus après récolte et du transport plutôt que celle des petits agriculteurs à fournir des produits de qualité moins chers. Les petits agriculteurs peuvent certes présenter un certain avantage concurrentiel dans une série de marchés axés sur la qualité (produits biologiques, par exemple), pour les processus intensifs en main-d'œuvre notamment, mais les conditions de participation au marché décrites plus haut dans les filières coordonnées verticalement et dominées par des normes privées les empêchent souvent de mettre à profit leur « avantage commercial».

Toutefois, ainsi que Swinnen (2005) le fait remarquer, les observations sont peu concluantes en ce qui concerne le degré d'exclusion de ces petits agriculteurs. Si la plupart des études concluent à l'existence d'une dynamique d'exclusion, il en est d'autres qui évoquent le potentiel d'inclusion que présentent les filières axées sur la qualité dans les pays en voie de développement (Giovannucci, 2003 ; Ponte et Gibbon, 2005). Les paragraphes qui suivent proposent d'explorer et de discuter plus avant les diverses possibilités d'inclusion qui pourraient être apportées par les innovations de marché axées sur la qualité.

\section{Éclairages sur les possibilités d'inclusion des mécanismes contractuels : considérations concernant les processus d'apprentissage}

L'importance croissante des normes et des mécanismes de coordination au sein des filières s'est avérée certes un obstacle pour les petits agriculteurs, mais également une opportunité de s'engager dans un processus d'apprentissage susceptible de les aider à améliorer leur accès aux marchés. En effet, les caractéristiques techniques relatives aux normes présentent les informations sous une forme codifiée et regroupée (Fulponi, 2006b). En outre, le recours aux normes peut simplifier la gestion des informations concernant la qualité des produits et rendre moins laborieux le fait de s'approvisionner auprès d'un grand nombre de petits agriculteurs (Chemnitz, 2007). En consolidant les liens entre les acteurs, les filières coordonnées sont en mesure de développer les capacités des petits producteurs à comprendre les exigences des acheteurs et les nouveaux besoins, et à s'y conformer.

Sur ce sujet, la présentation de Bolo au symposium ISDA sur la filière de la fleur coupée au Kenya apporte des éléments intéressants (encadré 6.1). Elle illustre certaines des limites du rôle joué par l'instauration de partenariats et la contractualisation des rapports entre les exportateurs et les petits planteurs dans le renforcement des capacités de ces derniers. Bien que ces mécanismes aient permis aux petits producteurs d'accéder au marché, et qu'ils aient ainsi contribué à abaisser les barrières les excluant d'une importante filière exportatrice de produits à haute valeur ajoutée, l'auteur indique que la relation est à sens unique - des directives et des instructions strictes confinant presque entièrement les planteurs aux seules activités de production. Les contrats d'approvisionnement prévoient certes une formation, et les exportateurs aident les planteurs à renforcer leurs capacités à se conformer aux 
exigences requises, mais cette formation se limite essentiellement aux opérations de production proprement dites, et les exportateurs ne divulguent généralement pas leur savoir concernant les activités génératrices de valeur ajoutée (les opérations après récolte et la vente). De telles dispositions empêchent les planteurs de prendre part à ces activités.

Encadré 6.1. Apprendre à exporter : partenariats agriculteurs-exportateurs au Kenya

\section{Maurice Ochieng Bolo}

Les partenariats contractuels entre petits agriculteurs et exportateurs sont considérés nécessaires pour renforcer les capacités des premiers tout en accordant aux seconds un contrôle pour garantir la livraison en temps et en heure de produits de haute qualité. Dans le cadre de cette étude, 116 agriculteurs/exportateurs ont tout d'abord été interrogés à l'aide d'un questionnaire structuré, puis trois cas particuliers de partenariats contractuels en cours ont été sélectionnés et examinés afin de décrire en détail la manière dont les institutions et les systèmes de gouvernance influencent les compétences des agriculteurs. Selon les résultats de cette enquête, les agriculteurs ont estimé l'accroissement de leurs compétences résultant de ces partenariats à $58,6 \%$ pour les capacités de production, à $23,3 \%$ pour les capacités de commercialisation, et à $18,2 \%$ seulement pour les capacités de génération de valeur ajoutée. Ce résultat est attribué aux dispositions institutionnelles établies dans ces contrats.

\section{Des contrats comme institutions}

Ces partenariats « agriculteur-exportateur » se heurtent à plusieurs obstacles, dont notamment les surcoûts de transaction et de coordination que les exportateurs doivent assumer du fait du nombre élevé de petits fournisseurs avec lesquels ils travaillent, les risques de comportement opportuniste auxquels sont exposés les exportateurs de la part des agriculteurs, et la possibilité que des concurrents viennent s'approvisionner auprès de fournisseurs compétents déjà formés par un autre exportateur. Ces enjeux maintiennent les exportateurs sous une pression permanente, d'où la nécessité pour eux de s'assurer que des mesures de protection sont en place. Cette protection est assurée par les institutions - les règlements, les lois, les normes et les codes de conduite qui régulent les comportements - telles qu'inscrites dans les contrats.

\section{Un double verrouillage}

Les dispositions de ces contrats montrent que les exportateurs doivent apporter une formation aux agriculteurs en ce qui concerne la production de «fleurs de bonne qualité ». Cette formation couvre les opérations qui vont de la plantation à la récolte, mais non celles qui interviennent après la récolte, qui sont réservées aux exportateurs. De même, les clauses liées aux activités qui génèrent de la valeur ajoutée, notamment le tri et le calibrage, la mise en bouquets et le conditionnement, ne concernent que les exportateurs. Les agriculteurs ne se chargent que du tout premier tri, sous le contrôle des exportateurs. Le reste des opérations de création de valeur ajoutée se déroule au sein des installations des exportateurs, où les fleurs sont ensachées et soumises à des solutions de pré-traitements avant d'être emballées et conditionnées pour l'exportation. Afin de maintenir « captifs » les agriculteurs, les contrats interdisent à ces derniers d'établir d'autres contrats avec d'autres exportateurs pour la fourniture du même produit. 
Les opérations post-récolte, la production de valeur ajoutée et les personnalisations à la demande de la clientèle sont des paliers qui font obstacle à la progression des petits agriculteurs. Ces étapes correspondent à un savoir « spécialisé » détenu par les exportateurs, qui possèdent également les infrastructures techniques nécessaires à cette production de valeur ajoutée. Comme l'apprentissage se fait par degrés successifs (Cohen et Levinthal, 1990) et que l'accroissement des compétences est source de compétitivité (Teece, 1998), de réduction des coûts et d'amélioration du niveau de performance et de fiabilité (Levinthal et March, 1993), les connaissances acquises au sein de ces partenariats constituent les « sites récepteurs » susceptibles d'accueillir les connaissances approfondies du futur. Il semblerait cependant que les petits agriculteurs ne soient pas en mesure de développer des "sites récepteurs » pour le savoir relatif à la création de valeur ajoutée, et qu'ils finissent enfermés dans la production, creusant encore ainsi le fossé de leur marginalisation (Bolo, 2010).

D'autres contributions au symposium ont également souligné le caractère incomplet du processus d'apprentissage qui découle des rapports contractuels entre les petits agriculteurs et les acteurs aval de la filière, y compris lorsque les seconds font preuve d'un certain engagement en faveur de la démarginalisation des premiers par le biais d'une contractualisation à long terme (voir Dulcire, 2010, au sujet des plantations de cacao à Saõ Tomé). Dulcire (2010) s'interroge sur la capacité des petits producteurs à renforcer leurs compétences en matière de production et de commercialisation et à devenir moins dépendants des interventions extérieures. Selon cet auteur, bien que les acteurs de l'aval soient intéressés par un approvisionnement de qualité auprès de petits producteurs dans le cadre plus large d'une stratégie de labellisation de la qualité (produits biologiques ou du commerce équitable), le fait que les termes du contrat soient en grande partie définis à l'extérieur puis imposés nuit beaucoup à la capacité des producteurs à s'approprier le processus, d'où un apprentissage tronqué, tant sur le plan technique qu'organisationnel.

\section{Éclairages sur les possibilités de modifications des règles commerciales}

Comme l'a montré Altenburg (2006), certaines modifications apportées aux règles et aux conditions qui régissent l'approvisionnement de filières engagées dans une démarche de qualité sont en elles-mêmes susceptibles de favoriser les petits agriculteurs.

\section{Les règles du commerce équitable}

Loconto et Simbua se sont penchés sur les répercussions des règles du commerce équitable dans les filières du thé en Tanzanie. Ces auteurs s'interrogent sur le réel degré d'influence des règles du commerce équitable sur la gouvernance des filières du thé en Afrique. Leurs résultats, qui ne corroborent pas les affirmations d'Altenburg (encadré 6.2), sont intéressants en ce que les règles du commerce équitable sont justement sensées changer les règles du marché au profit des petits agriculteurs. Or ils montrent que la capacité des petits producteurs à obtenir et à maintenir leur 
participation aux filières du commerce équitable doit plus aux systèmes d'organisation spécifiques pré-existants qui régissent les rapports, au sein de ces filières, entre ces petits producteurs et les usines de transformation, qu'aux attributs mêmes de ces filières qui sont sensés découler des principes et des règles sous-jacents à la certification équitable (prix de vente élevé, investissement dans le développement, possibilité de paiements à l'avance, engagement à long terme par le biais d'une contractualisation). Si l'engagement à long terme des parties prenantes favorise des rapports plus équilibrés et plus durables entre les planteurs et les transformateurs, les rapports de propriété ne sont pas déterminants pour la pérennité de la participation aux filières de commerce équitable. La participation durable au sein de ces filières semble donc être plus liée à des rapports équilibrés pré-existants qu'aux dispositifs conçus par le commerce équitable pour développer une participation plus équitable des petits agriculteurs.

Lev et Stevenson (2010) montrent toutefois, aux États-Unis, l'intérêt commun que des exploitations agricoles de taille moyenne et des entreprises agroalimentaires travaillant à une échelle régionale peuvent avoir dans l'instauration de modèles commerciaux « équitables » qui leur sont propres. Ces modèles consistent en des alliances stratégiques fondées sur des notions d'équité, sur la transparence et sur des identités de marque communes (itinéraire particulier des denrées produites) qui entretiennent la confiance et le soutien des consommateurs. Dans un tel contexte, des règles de «commerce équitable » sont élaborées en interne entre les agriculteurs et les entreprises agroalimentaires, dans le cadre d'une démarche visant à développer certains aspects qualitatifs pour accroître le caractère distinctif de leurs produits et leur compétitivité sur les marchés régionaux. Bien que ces modèles ne ciblent pas particulièrement les petits agriculteurs, ils permettent à ceux qui ne sont ni compétitifs sur les marchés internationaux ni adaptés à une commercialisation directe à l'échelle locale de poursuivre leur activité et de contribuer ainsi à la durabilité sociale (vitalité de la communauté) et environnementale (structure diversifiée et résiliente de l'agriculture). Ces modèles s'appuient sur l'action collective, à la fois horizontalement, entre agriculteurs, et verticalement, entre ces derniers et l'entreprise aval.

\section{Les mécanismes de certification}

Les contributions présentées au symposium ISDA permettent par ailleurs de mieux comprendre la manière dont les mécanismes et les règles de certification influencent les dynamiques sociales liées aux marchés, et donc leur potentiel en matière d'insertion des petits agriculteurs. Ainsi Sabourin (2010) montre-t-il que les programmes de certification faisant intervenir des processus collectifs (co-certifications, certifications de groupes et mécanismes participatifs de certification) peuvent contribuer à réintroduire une certaine réciprocité dans le "système capitaliste de marché d'échange » et, par conséquent, à soutenir des types différents de dynamiques sociales fondées sur le marché. Il montre en particulier que ces programmes offrent de meilleures opportunités pour prendre en considération et valoriser les spécificités des petits agriculteurs.

D'autres intervenants au symposium ISDA considèrent par ailleurs qu'il convient de s'interroger sur les capacités effectives des divers mécanismes reposant sur la 


\section{Encadré 6.2. Les normes du commerce équitable comme moyens d'innover ? Allison Loconto et Emmanuel Simbua}

Ce cas interroge le degré d'influence réel des règles du commerce équitable sur la gouvernance au sein des filières de production en Afrique, et plus précisément sur l'objectif ultime du commerce équitable, à savoir la démarginalisation des petits producteurs. En s'appuyant sur des données provenant du secteur du thé en Tanzanie, Loconto et Simbua ont examiné les systèmes d'organisation régissant les rapports entre les petits producteurs et les usines de transformation faisant partie des filières certifiées « équitable ». D'après leurs résultats, plusieurs modalités existent en matière de propriété au sein de ces filières certifiées et opèrent d'une manière relativement indépendante du système de certification « équitable ». En effet, les rapports anciens et institutionnalisés entre la direction des usines de transformation et les petits planteurs de thé influencent, positivement ou négativement selon les cas, l'intégration de ces producteurs aux marchés du commerce équitable.

Dans la pratique, Loconto et Simbua relèvent que les usines de transformation qui font partie du réseau "équitable » sont celles qui s'étaient déjà engagées dans un mouvement général d'innovation organisationnelle dans le cadre de l'adoption de modèles commerciaux plus généraux. Les innovations en question incluent notamment la propriété conjointe des usines de transformation par des coopératives de petits planteurs de thé et des investisseurs privés, et la négociation collective, pour chaque usine, de contrats la liant aux producteurs qui la fournissent. Ces contrats comprennent des subventions aux moyens de production et des crédits pour les planteurs, et, de la part des usines, la garantie d'un paiement au moment dû. Qui plus est, les prix négociés à l'échelle locale sont fortement influencés par les rapports de force locaux, dans lesquels les coopératives de petits cultivateurs ont plus de poids que ce qui est communément admis. Enfin, les auteurs ont trouvé que, sur les quatre situations étudiées, une seule concernait une association de petits planteurs qui avait su conserver sa certification « équitable ».

Les auteurs suggèrent de ce fait qu'une innovation organisationnelle permettant effectivement une plus grande participation des parties prenantes aux prises de décision et au contrôle des ressources est importante pour la conservation de la certification équitable. Cependant, ils révèlent également que les bénéfices perçus du commerce équitable n'augmentent pas nécessairement avec le nombre de parts de l'usine de transformation qui sont possédées : il semble plutôt que ce soient les rapports à long terme qui existaient entre les acteurs de la filière et les autres parties prenantes qui ont su façonner des relations plus durables entre planteurs et transformateurs dans le secteur du thé. La plupart des caractéristiques des filières du commerce équitable (telles qu'un meilleur prix de vente, un investissement dans le développement, la possibilité de paiements à l'avance, des engagements contractuels à long terme) existent déjà dans certaines filières de production de thé en Tanzanie. Pour résumer, il apparaîtrait que ce soient ces conditions pré-existantes qui facilitent la participation aux filières du commerce équitable plutôt que l'inverse (Loconto et Simbua, 2010).

qualité et les normes à refléter, exploiter et valoriser la diversité des systèmes et des conditions de production. Mateos et Ghezán (encadré 6.3) insistent sur l'importance de prendre ces problèmes en considération dans le cadre des processus nationaux de réglementation concernant les normes de l'agriculture biologique en comparant les cas de l'Argentine et du Brésil. Elles étudient la trajectoire argentine en matière 


\section{Encadré 6.3. Construction sociale des normes dans l'agriculture biologique et inclusion des petits agriculteurs en Argentine}

\section{Mónica Mateos et Graciela Ghezán}

Dans le cas des produits issus de l'agriculture biologique, l'analyse de la construction sociale du processus de qualité permet de mieux comprendre la coexistence de conventions multiples (Busch, 2004). Les normes commerciales, mises au point par le biais de forums hybrides (Callon, 2002) ou de réseaux socio-techniques, mettent en évidence les controverses qui existent au sujet de l'inclusion des petits agriculteurs.

Le cas de l'Argentine illustre la prééminence des considérations de marché sur celles relatives à l'inclusion des petits producteurs dans la réglementation concernant les normes de l'agriculture biologique. La majorité de la production agricole biologique du pays (95\%) est vendue sur le marché international, l'Argentine étant le premier pays tiers à bénéficier d'une certification de l'Union Européenne pour sa production biologique. Pendant les années 1990, les exigences légalement requises pour la certification d'un pays tiers ne tenaient pas compte des difficultés éprouvées par les petits agriculteurs pour adapter leurs pratiques de production et mettre en application les nouveaux procédés.

Il est intéressant de constater que les réglementations en matière d'agriculture biologique en Amérique latine ont évolué en quelques années. Initialement, la dynamique brésilienne était l'exact opposé de celle observée en Argentine, des mouvements sociaux et des organisations non gouvernementales (ONG) tentant d'emblée d'inclure les petits producteurs et de prendre en considération les préoccupations des consommateurs locaux en développant une agriculture écologique adaptée aux conditions locales. Sur ce point, le Brésil ne se contente pas de prendre en compte les considérations de marché imposées par les marchés internationaux (Meirelles, 2003). Les transformations récentes sont principalement liées aux contextes sociaux, économiques et politiques différents. Au cours des dernières années, un surcroît d'informations et de connaissances est apparu au sujet de plusieurs expériences incluant des petits agriculteurs. Ces cas d'inclusion, hétérogènes, vont du commerce équitable au développement d'une agriculture écologique.

En Argentine aujourd'hui, ainsi que le met en évidence l'analyse des négociations concernant les différentes dimensions de la qualité dans les produits de l'agriculture biologique, la promotion récente de l'agriculture familiale, basée sur le développement durable et sur la diffusion de processus de certification collective, ouvre un nouveau débat autour des normes et des règles qui étaient en vigueur jusqu'ici.

Ainsi, dans le cadre du processus de modification de la législation sur l'agriculture biologique, l'objectif est d'inclure les petits producteurs par le biais de diverses approches, telles que la modulation du type de certification requis en fonction des marchés (domestique ou international) et l'inclusion de nouvelles formes de certification participative (à travers des mouvements sociaux ou des associations de producteurs) avec une participation variable de l'État selon les cas. La direction et la rapidité de ces évolutions sont liées aux capacités de ces acteurs à faire la jonction avec les réseaux innovants nationaux ou régionaux pour ce qui concerne tant les aspects de production que d'organisation (Mateos et Ghezán, 2010).

de réglementation et le changement d'optique qui a été amené par l'évolution de la dynamique sociale. Leur analyse de la réglementation des normes relatives à l'agriculture biologique en Argentine incite à s'interroger sur les parties prenantes 
qui participent à l'élaboration des politiques et sur la nature de la réglementation publique qui régit les activités des intervenants et le fonctionnement du marché. Ceci souligne la nécessité de ne pas considérer l'État et le marché comme des forces opposées mais plutôt comme des dimensions étroitement imbriquées.

\section{" Conclusion}

Ce chapitre a été principalement consacré aux innovations axées sur le marché et aux innovations de filière dans les systèmes agroalimentaires. Ces innovations se sont développées de manière significative au cours des dernières années, en même temps qu'une dynamique de la qualité et le développement des normes. Elles sont étroitement associées à des formes de plus en plus sophistiquées d'intermédiation et de coordination au sein des filières, ce qui a un impact sur la gouvernance des filières et des conséquences significatives pour ce qui est des aspects sociaux de la durabilité. Ainsi qu'il a été montré dans ce chapitre, le potentiel des innovations de marché pour initier des changements favorisant une insertion croissante et prolongée des petits agriculteurs dépend beaucoup de qui contrôle ou porte ces innovations. Leurs capacités à améliorer la participation des petits producteurs est considérablement limitée par l'asymétrie structurelle du pouvoir, qui est encore très largement répandue. Dans bien des cas, les innovations qui concernent le marché ou les questions de qualité se sont traduites par un renforcement des positions dominantes. Les innovations de marché sont certes susceptibles de valoriser des pratiques ou des ressources locales en tant qu'avantages commerciaux et de faciliter ainsi, en théorie, l'accès des petits agriculteurs au marché, mais cela dépend dans une très large mesure des types de processus à l'œuvre, et notamment des possibilités d'instaurer des relations plus équilibrées. Plusieurs dimensions se sont ici révélées importantes, en particulier : les possibilités d'apprentissage et d'autonomisation (tant sur le plan technique qu'organisationnel) et la nature des processus impliqués ; la nature des processus réglementaires relatifs à la certification et à la mise en place des normes ; et les aspects relatifs à l'action collective, qui se sont avérés une dimension sous-jacente décisive dans les différents cas étudiés. En sus des considérations de marché abordées dans ce chapitre, beaucoup des contributions au symposium ISDA ont enfin souligné le rôle clé de l'action collective autour du développement et de la signalisation de la qualité à l'échelon local pour tirer parti de ressources particulières (telles que des atouts territoriaux) par la co-construction de ressources territorialisées ou locales porteuses d'un message de qualité et de savoir-faire.

Ces trois dimensions - l'action collective et les dynamiques sociales sous-jacentes à l'élaboration des normes et des processus de qualifications, la diffusion des savoirs, et les règles régissant les processus réglementaires - doivent toutes être examinées d'un œil critique lorsqu'il s'agit de considérer les possibilités offertes par les diverses innovations de marché en matière d'inclusion des petits agriculteurs. Elles sont tout à la fois causes et conséquences de la nature et de la portée des marchés dans lesquels sont introduites ces innovations de filière, et notamment de l'échelle géographique de ces marchés (local, régional ou international) et des spécificités mises en valeur dans les filières (commerce équitable ou agriculture biologique, par exemple). 


\section{" Références bibliographiques}

Altenburg T., 2006. Symposium: shaping value chains for development. The European Journal of Development Research, 18.

Barcala M.F., Gonzalez-Diaz M., Raynaud E., 2007. The governance of quality: The case of the agrifood brand names. $3^{\text {rd }}$ International Conference on economics and management networks, Rotterdam School of Management, 28 - 30 juin, Erasmus University.

Berdegué J., Biénabe E., Peppelenbos L., 2011. Conclusions: Innovative practices in connecting small-scale producers with dynamic markets, In : Reconnecting markets: innovative global practices in connecting small-scale producers with dynamic food markets (Biénabe E., Berdegué J., Peppelenbos L., Belt J., eds), Gower Publishing, Royaume-Uni.

Bolo M.O., 2010. Learning to export: building farmers' capabilities through partnerships in kenya's flower industry, In : Innovation and Sustainable Development in Agriculture and Food (ISDA), Montpellier, France - http://hal.archives-ouvertes.fr/hal-00526145/en/

Busch L., 2004. Grades and standards in the Social Construction of safe food, In : The politics of food (Lien M.E., Nerlich B., eds), Berg, Oxford, Royaume-Uni.

Callon M., Meadel C., Rabeharisoa V., 2002. The economy of qualities. Economy and Society, 31(2).

Chemnitz C., Grethe H., Kleinwechter U., 2007. Quality standards for food products - A particular burden for small producers in developing countries? In : Pro-poor development in low income countries: food, agriculture, trade and environment, Colloque EAAE, 25-27 octobre 2007, Montpellier, France.

Codron J., Giraud-Héraud E., Solar L., 2005. Minimum quality standards, premium private labels, and European meat and fresh produce retailing. Food policy, 30.

Cohen W.M., Levinthal D.A., 1990. Absorptive capacity: A new Perspective on Learning and Innovation. Administrative Science Quarterly, 35 (1), 128-152.

Conférence des Nations Unies sur le Commerce et le Développement (CNUCED), 2007. Privatesector-set Standards and Developing Countries' Exports of Fresh Fruit and Vegetables: Synthesis of Country-case Studies in Africa (Ghana, Kenya, Uganda), Asia (Malaysia, Thailand, Viet Nam), and Latin America (Argentina, Brazil, Costa Rica). Note d'information par le Secrétariat de la CNUCED pour le Regional Workshop on Good Agricultural Practices in Eastern and Southern Africa: Practices and Policies, FAO-CNUCED, 6-9 mars 2007, Nairobi, Kenya.

Dolan C., Humphrey J., 2000. Governance and trade in fresh vegetables: the impact of UK supermarkets on African horticultural industries. Journal of Development Studies, 35, 147-177.

Dulcire M., 2010. La mise en place participative d'une filière cacao à Sao Tome. L'organisation des producteurs en tant que facteur d'émancipation, In : Innovation and Sustainable Development in Agriculture and Food (ISDA), Montpellier, France - http://hal.archives-ouvertes.fr/hal-00510555/

Farina E.M.M.Q., Gutman G.E., Lavarello P.J, Nunes J., Reardon T., 2005. Private and public milk standards in Argentina and Brazil. Food Policy, 10.

Food and Agriculture Organization (FAO), 2007. Private standards in the United States and European Union markets for fruit and vegetables - implications for developing countries. FAO commodity studies, www.fao.org.

Fulponi L., 2006a. Private Standards and the Shaping of the Agro-Food system. Rapport de l'OCDE, Paris, France.

Fulponi L., 2006b. Private voluntary standards in the food system: the perspective of major food retailers in OECD countries. Food Policy, 31.

Gibbon P., Ponte S., 2005. Trading Down: Africa, Value Chains and the Global Economy, Temple University Press, Philadelphia, États-Unis.

Giovannucci D., Reardon T., 2000. Understanding grades and standards and how to apply them, Banque Mondiale, Washington DC, États-Unis.

Giovannucci D., 2003. Emerging issues in the marketing and trade of organic products, In: Organic Agriculture: Sustainability, Markets and Policies, Séminaire de l'OCDE sur l'agriculture biologique, septembre 2002, OCDE, Paris, France. 
Hammoudi A., Hoffmann R., Surry Y., 2009. Food safety standards and agri-food supply chains: an introductory overview. European Review of Agricultural Economics, 36 (4), 469-478.

Hanf J.H., Pienadz A., 2007. Quality management in supply chain networks. The cases of Poland. International Food and Agribusiness Management Review, 10 (4), 103-128.

Havinga T., 2008. Actors in private food regulation: taking responsibility or passing the buck to someone else? In : Private Governance in the Global Agro-Food System, 23-25 avril 2008, Munster, Allemagne.

Henson S., Humphrey J., 2009. The impacts of private food safety standards on the food chain and on public standard-setting processes. Trente-Deuxième Session du Programme mixte FAO/OMS sur les normes alimentaires, Commission du Codex Alimentarius, FAO, 29 juin - 4 juillet 2009, Rome, Italie.

Henson S., Reardon T., 2005. Private agri-food standards: implication for food policy and the agrifood system. Food Policy, 30.

Lev L., Stevenson J., 2010. Acting collectively to develop midscale food value chains in the U.S., In : Innovation and Sustainable Development in Agriculture and Food (ISDA), Montpellier, France - http://hal.archives-ouvertes.fr/hal-00520462/

Levinthal D.A., March J.G., 1993. The myopia of learning. Strategic management journal, 14, 95-112.

Loconto A.M., Simbua E.F., 2010. Organizing smallholder production for sustainability lessons learned from fairtrade certification in the Tanzanian tea industry, In : Innovation and Sustainable Development in Agriculture and Food (ISDA), Montpellier, France - http://hal.archives-ouvertes.fr/ hal-00529061/fr/

Mateos M., Ghezán G., 2010. El proceso de construcción social de normas de calidad en alimentos organicos y la inclusión de pequeños productores el caso de Argentina, In : Innovation and Sustainable Development in Agriculture and Food (ISDA), Montpellier, France - http://hal.archivesouvertes.fr/hal-00566243/fr/

Meirelles L., 2003. La certificación de productos orgánico -caminos y descaminos, http://www.centroecologico.org.br/artigo_detalhe.php?id_artigo $=25$

Ménard C., Valceschini E., 2005. New institutions for governing the agri-food industry. European Review of Agricultural Economics, 32 (3).

Ponte S., Gibbon P., 2005. Quality standards, conventions and the governance of global value chains. Economy and society, 34 (1).

Raynaud E., Savée L., Valceschini E., 2002. Governance of the agri-food chains as a vector of credibility for quality signalization in Europe, In : Exploring diversity in the European Agri-food System, $10^{\mathrm{e}}$ congrès de l'EAAE, 28-31 août 2002, Zaragoza, Espagne.

Reardon T., Timmer C.P., Barrett C.B., Berdegue J., 2003. The rise of supermarkets in Africa, Asia, and Latin America. American Journal of Agricultural Economics, 85 (5), 1140-1146.

Ruben R., Slingerland M.A., Nijhoff H., 2006. Agro-food chains and networks for development: issues, approaches and strategies, In : Agro-food supply chains and networks for development, actes du séminaire de Frontis, Wageningen, Pays-Bas, 6-7 septembre 2004, Frontis-Kluwer/Springer Verlag, Wageningen UR-Frontis Series 14.

Sabourin E., 2010. Agri-food qualification and certification process as an interface between exchange marketing and reciprocity, In : Innovation and Sustainable Development in Agriculture and Food (ISDA), Montpellier, France - http://hal.archives-ouvertes.fr/hal_00521969/

Ssemwanga J., 2005. Présentation à la réunion de clôture de l'action concertée de l'Union Européenne High quality and safe food chains (SafeACC), 22 mai 2005, Buenos Aires, Argentine.

Swinnen J.F.M., 2005. When the market comes to you - or not: the dynamics of vertical coordination in agri-food chains in transition. In : Final Report of the World Bank (ECSSD) ESW on Dynamics of Vertical Coordination in ECA Agrifood Chains: Implications for Policy and Bank Operations, Banque Mondiale, Washington D.C., États-Unis.

Teece D.J., 1998. Capturing value from knowledge assets: the new economy, markets for know-how and intangible assets. California Management Review, 40 (3).

Teece D.J., Pisano G., Shuen A., 1997. Dynamic capabilities and strategic management. Strategic Management Journal, 18 (7), 509-533. 
Vorley B., 2001. The chains of agriculture: sustainability and the restructuring of agri-food markets. Document d'opinion de l'IIED et du RING préparé en vue du World Summit on Sustainable Development.

Wilson N., Van Ittersum K., Fearne A., 2000. Cooperation and coordination in the supply chain: a comparison between the Jersey Royal and the Opperdoezer Ronde potato. In: The socio-economics of origin-labelled products in agro-food supply chains: spatial, institutional and co-ordination aspects (Sylvander B., Barjolle D., Arfini F., eds), Série Actes et Communications, 17 (1), Inra, France. 


\title{
Chapitre 7 \\ Innovations et gouvernance des territoires ruraux
}

\author{
André Torre et Frédéric WaLleT
}

L'idée que l'innovation ou la créativité pourraient être à l'origine des processus de développement des territoires est apparue assez récemment dans la littérature, ainsi que dans les politiques et actions publiques. Ce n'est que ces dernières années que s'est imposé le fait que les activités nouvelles pouvaient présenter un intérêt, voire même constituer le moteur de la croissance des territoires (Regional Science Policy and Practice, 2011), et encore cette approche est le plus souvent circonscrite aux seuls secteurs de haute technologie ou de la nouvelle économie.

Il a fallu attendre les années 1990 pour voir apparaître des travaux qui mettent l'innovation au cour de la croissance des régions ou des territoires, et signalent la place importante tenue dans ce mécanisme par les entreprises innovantes, les clusters et les regroupements d'activités créatrices de haute technologie, la diffusion spatiale des technologies et ses limites géographiques en termes de spillovers, puis les problèmes de capacité d'absorption et de reproductibilité difficile des innovations produites ailleurs. Ce mouvement s'est accompagné de la mise en œuvre de nombreuses politiques locales, nationales ou communautaires en faveur de l'innovation, telles que la création de technopoles ou de parcs d'activités scientifiques, la dévolution de crédits importants en faveur de la recherche et développement, ou l'approfondissement du lien recherche/industrie. Dans tous les cas, c'est la conception de l'innovation high-tech qui est privilégiée, l'accent étant mis sur la création ou le transfert d'innovations de très haut niveau, devant profiter aux entreprises qui les utilisent ainsi qu'à leur réseau de sous-traitants, fournisseurs ou voisins géographiques, puis bénéficier par capillarité à l'ensemble de l'économie locale.

Le modèle de développement régional ou territorial qui en découle est axé sur les activités high-tech : l'innovation est considérée comme le principal moteur de la croissance (version édulcorée du développement), ainsi que comme un moyen de se différencier et donc de lever en partie la contrainte concurrentielle. Les institutions internationales (ODCE, UE...) et les gouvernements, qui prônent ces politiques de développement fondées sur la compétitivité et l'innovation, mettent en place des dispositifs visant à intensifier la sélection entre les territoires. Il en résulte alors souvent une certaine forme d'angélisme des gestionnaires des territoires, 
désireux d'entrer dans le jeu de la concurrence, qui considèrent que la valorisation des ressources locales et l'appui aux secteurs de pointe suffisent à générer du développement.

Mais les territoires ne jouent pas à armes égales dans cette course à l'excellence technologique : tous n'ont pas des ressources facilement valorisables, ni les compétences pour les valoriser. C'est notamment vrai pour les territoires ruraux, ou pour les pays du Sud, qui conduisent à reposer la question de la nature de l'innovation et des conditions de son émergence sur les territoires. Cet article a pour objet d'explorer le lien entre trois éléments essentiels : l'innovation, le développement territorial et la gouvernance. Dans une première partie, nous présentons les principaux modèles de développement et leurs déclinaisons dans les territoires ruraux ou agricoles. Nous abordons ensuite la question de la place de l'innovation dans les approches du développement, en considérant successivement les approches de l'innovation territorialisée et les politiques territoriales d'innovation. Nous terminons enfin par l'analyse des modes de gouvernance des espaces ruraux et périurbains, qu'il s'agisse des processus de délibération, des acteurs et structures de gouvernance ou des mécanismes respectifs de conflits et de concertation, considérés comme des expressions ou des vecteurs de l'innovation sur les territoires.

\section{" Les modèles de développement régional et territorial}

Qu'ils soient centrés sur les questions rurales et agricoles ou plus généralement attachés à définir les conditions de croissance et de succès des économies régionales, les travaux consacrés à la thématique du développement font la part belle à l'étude des mécanismes économiques. On constate facilement que l'intérêt pour les questions d'innovation, très mesuré pendant l'après-guerre ou les Trente Glorieuses, est maintenant devenu l'une des composantes majeures de ces approches, tant la thématique du développement est étroitement liée à celle de l'innovation, sous toutes ses formes. Trois grandes visions concurrentes du développement coexistent, correspondant à des présupposés de nature analytique forts, dans lesquels l'innovation est plus ou moins présente (Torre et Wallet, 2012).

\section{Le développement comme équilibre optimal}

On trouve tout d'abord les courants de pensée qui s'attachent avant tout à définir une balance des intérêts et des gains retirés par les différents acteurs locaux du processus de développement et à rechercher des principes qui permettront d'atteindre le maximum de satisfaction des parties prenantes. Appartiennent à ce groupe les approches fondatrices de la théorie néo-classique, qui envisagent une croissance homothétique fondée sur les inputs en termes de capital et de travail, ultérieurement étendus à un troisième intrant de nature plus technologique, le plus souvent la connaissance ou le volume des investissements en matière de recherche et développement (R\&D) (Solow, 2000). Dans ces approches, l'innovation est essentiellement considérée sous l'angle d'un input destiné à améliorer l'efficacité de l'affectation et de l'usage des facteurs de production, et leur productivité. Il s'agit d'évaluer le 
volume de production et sa croissance, et de les mettre en parallèle avec le caractère optimal de la combinaison de facteurs et les efforts réalisés en termes de productivité ou d'accumulation du capital par exemple (voir Johansson et al., 2001). Cette approche, qui considère la possibilité d'élimination à terme des disparités interrégionales, a connu un succès important mais relatif, principalement en raison de ses limites en termes de croissance homothétique et de son incapacité à rendre compte des déséquilibres signalés très tôt par les auteurs de la polarisation ou de la croissance par le bas par exemple.

\section{Le développement comme source d'inégalités et de polarisation}

Le deuxième bloc d'analyses, le plus important, est constitué par les approches qui pensent que les compromis passés entre les acteurs locaux sont purement provisoires et que les processus de développement génèrent des inégalités interrégionales difficilement réductibles. À rebours des précédentes, ces analyses considèrent que le développement accompagne et contribue à creuser les disparités entre régions ou territoires, souvent de manière durable. Elles soulignent également l'existence de systèmes locaux présentant des particularités marquées au niveau institutionnel, économique et technique, et dont les succès ou les échecs provoquent des processus de développement fondamentalement déséquilibrés. Ces travaux trouvent leur origine dans les analyses des pôles de croissance, initiées par Perroux, Myrdal, puis Hirschman ou Higgins. L'idée de départ de Perroux (1961) est que le développement ne peut pas survenir partout, au même moment, et avec la même intensité. La preuve en est l'existence de pays ou de zones en retard de développement, que la théorie des pôles de croissance a été la première à reconnaitre. Le développement repose sur un processus de polarisation des activités, lui-même fondé sur l'existence de grandes firmes motrices, situées au cœur des régions les plus développées, et qui sont le vecteur de l'innovation et de sa diffusion déséquilibrée entre les territoires.

Avec la crise du fordisme et l'incapacité des modèles traditionnels à rendre compte des évolutions du capitalisme, comme de la performance de formes d'organisation alternatives au modèle de la grande firme, ont émergé des analyses mettant les facteurs intangibles au cour des dynamiques de développement. Ainsi, Porter (Porter, 1985, 1990), dont les approches ont connu un large retentissement, explique l'avantage compétitif d'une région ou d'un territoire à partir de quatre grands facteurs qui doivent être travaillés dans le but de procurer une avance sur les zones concurrentes : les stratégies, structures et rivalité (rivalry) des entreprises, l'état de la demande, les relations spatiales entretenues avec les industries liées, et l'état des ressources ou facteurs de production (traditionnels ou en termes de compétences). Les analyses en termes d'économie résidentielle ou présentielle, qui fondent le développement territorial sur la captation de revenus extérieurs, présentent une autre illustration des disparités interrégionales (Davezies, 2008).

L'analyse des systèmes locaux de production (SPL), qui prend naissance dans les années 1970, repose également sur la constatation de processus de développement spatialement différenciés. Initiée par les analyses des districts italiens (Beccatini, 1990), puis par une série de déclinaisons allant des milieux aux clusters en passant par les systèmes agro-alimentaires ou les SPL, elle est fondée sur le caractère 
systémique des relations entretenues par les acteurs qui appartiennent et dessinent ensemble un territoire fondé sur leurs relations de coopération et leurs projets communs. On retrouve ici l'idée du développement par le bas, chère à des auteurs comme Stohr (1986) ainsi qu'une volonté de typologisation des formes de développement (les districts italiens, les systèmes fondés sur le public, les systèmes avec un cœur de grandes firmes, ou fondés sur l'innovation...) (Markusen, 1996), mais peu d'analyse des processus même de développement et de leur dynamique.

La nouvelle économie géographique, initiée par Krugman (1991) et popularisée par des auteurs tels que Fujita, Thisse ou Ottaviano par exemple (Fujita et Thisse, 1997, 2001 ; Ottaviano et Thisse, 2004), a ensuite formalisé la probabilité importante de survenance de phénomènes de polarisation spatiale et de concentration des activités. Se posent les questions des capacités d'entraînement d'une activité au niveau régional (par exemple l'effet d'entraînement de la construction), de l'impact réciproque de la localisation des entreprises et de celle des travailleurs-consommateurs, ainsi que de la maitrise de la diminution des coûts de transport, qui ne fait que renforcer les processus de polarisation au détriment des zones périphériques.

\section{Le développement comme processus dynamique lié à l'innovation}

Un troisième et dernier bloc de recherche repose sur l'idée que le développement régional ou territorial est profondément lié à l'existence de ruptures dynamiques, résultant de processus d'innovation ou de création, à l'origine de rythmes et de volumes de développement différents selon les régions ou les territoires (Dunford, 1993 ; Scott et Storper, 2003). Les analyses du développement régional fondées sur les processus d'innovation et de régulation, ainsi qu'une partie des approches systémiques considèrent ainsi que les systèmes locaux sont soumis à des phases successives de croissance et de stagnation, voire de récession (Colletis et al., 1999), qui aiguisent ou réduisent les inégalités entre catégories sociales, les fruits de la croissance pouvant se voir appropriés par certains groupes ou par des entreprises off-shore aux mains de capitaux extérieurs. Ce sont avant tout les chocs internes qui génèrent des transformations du système et conduisent à l'apparition de processus de concentration spatiale des personnes et des richesses, ainsi que de zones d'exclusion sociale et spatiale. L'innovation, sa création et sa diffusion se trouvent alors au cour de ces approches (Cooke et Morgan, 1998).

Au cours de la dernière décennie, l'analyse des dynamiques spatiales s'est enrichie de travaux ancrés dans la filiation de la théorie évolutionniste (Frenken et Boschma, 2007), qui considèrent la répartition inégale des activités dans l'espace comme le résultat de processus historiques largement contingents. L'économie géographique évolutionniste accorde une place prédominante à la dimension entrepreneuriale, qu'il s'agisse de la généalogie ou des processus d'émergence, de croissance, de déclin et de cessation d'activité des entreprises (Boschma et Frenken, 2011). L'accent est mis particulièrement sur le rôle joué par les spin-off et la mobilité de la main d'œuvre dans les processus de développement territorial (Maskell, 2001), ainsi que sur le mécanisme de réplication des routines au sein du tissu industriel local. Tirant avantage des proximités géographique, industrielle et technologique entre secteurs 
d'activités (Torre, 2008), ainsi que des dispositifs institutionnels et des structures des réseaux, ces technologies se diffusent par effet boule de neige entre les entreprises et industries technologiquement liées, et finissent par verrouiller les systèmes locaux dans des dépendances spatiales aux sentiers de croissance. Ce processus fonctionne particulièrement bien quand les industries sont émergentes ou reposent sur des technologies connexes, la faible distance cognitive favorisant la circulation des externalités de connaissance (Nooteboom, 2000).

\section{" Les politiques de développement par l'innovation}

Une des caractéristiques des politiques de développement contemporaines est de considérer que le dynamisme local en matière d'innovation, de production et de transfert des connaissances constitue l'une des clés du développement régional, d'où l'effort important des régions et collectivités locales dans ce domaine. Les politiques de soutien à l'innovation, source de rendements croissants, font aujourd'hui partie de l'arsenal des décideurs, qui y voient l'argument suprême en matière de croissance ou de développement (Hall, 1994). Elles reposent sur la constatation de la difficulté à s'approprier les gains de l'innovation, qui appelle une intervention de l'État pour compenser un possible sous-investissement en matière de R\&D. Ces stratégies, qui ont donné naissance à des politiques de promotion des activités high-tech (Goldstein, 2009) ou de grands programmes industriels de type Airbus, sont également considérées comme pertinentes pour les espaces ruraux, les régions isolées et les PME qui manquent de ressources.

\section{L'innovation technologique au sein de pôles de développement}

Les approches traitant du rôle de l'innovation dans les dynamiques de développement territorial et régional sont fondées sur la prise en compte de l'importance des activités de R\&D ou d'innovation en matière de développement local. En partie inspirées des analyses de Schumpeter, elles reposent sur l'idée que les innovations constituent la clé des processus de développement et que les efforts réalisés en matière de $R \& D$ ou d'incitations à innover peuvent jouer un rôle important dans la mise en place et le succès de dynamiques de croissance. Il s'agit souvent d'une approche systémique, qui souligne le rôle joué par le transfert et la diffusion des innovations au niveau local (Feldman, 1994 ; Autant-Bernard et al., 2007), ainsi que l'importance des relations de face à face et des phases d'essaimage par installations de spin offs ou d'accompagnement de l'effort de création (nurseries, hôtels de projets...). Le moteur du développement réside alors dans l'existence de spillovers localisés d'innovation ou de connaissances, qui se diffusent au sein du système local et peuvent donner naissance à des systèmes locaux d'innovations très compétitifs, du type technopoles ou pôles de compétitivité par exemple. C'est l'innovation qui tire le développement, et marque la différence entre les systèmes dynamiques et les autres.

La concentration des efforts d'investissement industriel sur des pôles clairement identifiés est aujourd'hui dominante dans les politiques européennes, mais une de 
ses limites repose sur sa conception linéaire, qui minimise l'importance des boucles de rétroaction et de l'incertitude dans les processus d'innovation. Elle conduit à des résultats assez pauvres, dans la mesure où elle omet la concentration géographique de la $R \& D$ et de l'innovation dans un petit nombre de régions ainsi que le phénomène d'exploitation des nouveaux savoirs hors des zones soutenues. De plus, les politiques de « choix du gagnant » (picking-the-winner policy) visant à sélectionner les zones les plus propices à l'innovation et les secteurs les plus dynamiques en termes de création d'emplois futurs (biotechnologie, nanotechnologies) peuvent voir leur utilité et leur pertinence interrogées (Boschma, 2009). Outre le fait qu'il est impossible de prédire les futures régions de croissance ou secteurs gagnants car les nouvelles industries sont souvent le résultat de processus spontanés plutôt que d'interventions orchestrées, elles conduisent à opter partout pour les mêmes activités, alors que les systèmes industriels et d'innovation sont très différents et souvent lacunaires (Camagni, 1995). Les phénomènes d'inertie et de lock-in conduisent ainsi la grande majorité des régions à échouer dans le développement de ces industries, entrainant des pertes énormes de ressources publiques.

Ces analyses prennent appui sur les changements de perception des processus d'innovation, passant du modèle linéaire au modèle interactif (Lundvall, 1992). Là où le modèle linéaire, inspiré de l'organisation taylorienne de la production, décrivait l'innovation comme un processus univoque cheminant de l'idée initiale à la production et à la commercialisation, le modèle interactif met l'accent sur le caractère interactif et itératif de l'innovation, entre des organisations étroitement liées aux différents stades de son développement. L'innovation est alors considérée comme une construction sociale prenant forme au sein d'une diversité de configurations géographiques (Wolfe et Gertler, 2002). Le modèle linéaire décrivait une division spatiale du travail fondée sur une spécialisation hiérarchique fonctionnelle, certaines régions bénéficiant des effets positifs, en matière de revenus et de croissance, du fait de leur positionnement et de leur spécialisation dans les activités de R\&D. A contrario, le modèle interactif met davantage l'accent sur les relations étroites entre utilisateurs et producteurs de connaissances, par le biais de leur proximité géographique et/ou des TIC. Par conséquent, les contextes institutionnels territoriaux sont essentiels pour expliquer le potentiel et les performances en matière d'innovation (Puttilli et Tecco, 2010 ; Bonaudo et al., 2010), certains territoires s'avérant nettement supérieurs à d'autres pour produire et adapter des innovations (Malecki, 1997).

La question de l'échelle à laquelle se déploie le processus d'innovation en lien avec les dynamiques de développement constitue également un élément essentiel du débat. À partir des travaux en termes de systèmes nationaux d'innovation (Lundvall, 1992 ; Nelson, 1993 ; Freeman, 1995 ; Amable et al., 1997), des réflexions sur leurs déclinaisons régionales ont cherché à comprendre à quelles conditions les réseaux et dispositifs institutionnels locaux et régionaux étaient plus ou moins favorables à l'innovation et quelles étaient les conditions de leur adaptation et de leur permanence dans le temps (Lundvall et Maskell, 2000). Il en a résulté des approches en termes de systèmes régionaux d'innovation (Cooke et Morgan, 1998), qui s'intéressent aux formes d'ancrage des innovations sur les territoires et tentent de mettre en évidence les conditions conduisant à des systèmes performants. Ces recherches insistent sur l'importance de la présence d'éléments tels que les infrastructures physiques et 


\section{Encadré 7.1. Les systèmes agroalimentaires localisés (Syal)}

L'approche en termes de Syal prend naissance à la fin $\mathrm{du} \mathrm{Xx}^{\mathrm{e}}$ siècle suite au constat de l'organisation des échanges et relations entre les acteurs locaux impliqués dans une activité de production agricole ou agro-alimentaire. Cette notion désigne « des organisations de production et de service (unité d'exploitation agricole, entreprises agroalimentaires, entreprises commerciales, entreprises de restauration...) associées de par leurs caractéristiques et leur fonctionnement à un territoire spécifique. Le milieu, les produits, les hommes, leurs institutions, leurs savoir-faire, leurs comportements alimentaires, leurs réseaux de relations se combinent dans un territoire pour produire une forme d'organisation agroalimentaire à une échelle spatiale donnée » (Muchnik, 1996).

Le concept, qui a rapidement connu un succès important auprès d'une partie de la communauté scientifique mais également auprès de décideurs et de pouvoirs publics (Muchnik et de Sainte Marie, 2010), constitue bien évidemment une extension de la notion de systèmes localisés de production et d'innovation et autres clusters aux dimensions de production agroalimentaire. Les Syal se rencontrent souvent dans des zones rurales, en particulier dans celles de pays en voie de développement, où l'organisation locale des acteurs du processus de production des aliments repose sur des liens locaux et la mise en commun de savoir-faire et de techniques (Sanz Cañada, 2010).

Par ailleurs, on note des références importantes à la dimension technique de production des biens, qui se trouve en relation étroite avec le fait social. On ne peut prendre en compte les modes de production et les techniques afférentes sans considérer les modalités d'organisation sociale des acteurs, ainsi que la construction conjointe des liens sociaux et des déterminants techniques dans l'action. On notera toutefois que ces systèmes restent encore orphelins d'une véritable appréciation en termes d'analyse : insuffisamment fondés, ils révèlent tout leur intérêt, sans que l'on soit encore capable de les rattacher à une approche théorique déterminante ou dominante, même si les pistes en termes d'économie de proximité ou de biens clubs pourraient donner lieu à des développements intéressants dans les années à venir (Perrier-Cornet, 2009).

technologiques, les liens entre industrie et universités en matière de $\mathrm{R} \& \mathrm{D}$, le haut niveau de qualification de la main d'œuvre sur le marché local du travail, l'existence de dispositifs de capital risque, mais aussi sur des facteurs moins tangibles liés au contexte social local, comme l'existence de savoir-faire et d'une culture technique régionale ou une proximité des cadres cognitifs collectifs. Le rôle des dispositifs institutionnels régionaux et locaux se révèle donc essentiel pour réduire l'incertitude et soutenir la coordination et l'action collective favorable aux processus d'innovation, les systèmes performants étant ainsi caractérisés par un haut niveau d'interactions locales et de relations d'interdépendance, où l'innovation est soutenue par des organisations publiques ou privées.

\section{L'innovation par la création de connaissances}

Des travaux plus récents soulignent le rôle central joué par la connaissance et ses implications en matière de développement territorial et régional, en lien avec 
les processus d'innovation. Selon cette lecture, le développement pourrait se comprendre comme le passage d'un ensemble d'actifs composé de produits faiblement élaborés et exploités par une main d'œuvre peu qualifiée, à un ensemble d'actifs basés sur la connaissance et exploités par une main d'œuvre qualifiée, l'information étant alors considérée comme une matière première primordiale (Lundvall et Maskell, 2000). La capacité d'apprentissage se révèle ainsi essentielle au potentiel d'adaptation des territoires et des régions pour leur développement, l'apprentissage étant considéré comme un processus collectif, social et géographique qui induit un changement dans les capacités ou la compréhension des individus ou des organisations.

Certains travaux insistent davantage sur la tension entre représentation et décision individuelle et innovation collective, mettant au centre de l'analyse les processus de création et de diffusion de connaissances. Dans cette perspective, les approches en termes de communautés de pratiques ancrées territorialement apportent notamment une grille conceptuelle originale mettant en exergue l'importance des routines et des réseaux, et se rapprochant des travaux en termes de ville créative (Cohendet et Simon, 2008), et d'économie géographique évolutionniste (Frenken et Boschma, 2007).

Enfin, les relations d'interdépendances non marchandes entre institutions sont déterminantes dans les performances des territoires et des régions en matière d'innovation, de croissance de la productivité et de développement. Les relations de confiance, mais aussi des niveaux élevés de connaissances tacites et l'existence de routines, structurent des dispositifs locaux de conventions et de coordinations spécifiques, constituant autant de ressources relationnelles favorables à l'augmentation des capacités d'apprentissage et à l'établissement d'avantages difficilement reproductibles par d'autres territoires. Dans cette perspective, les espaces d'agglomération et plus largement les territoires urbains sont considérés comme favorables à l'innovation et à la création de connaissances en raison des externalités cognitives qu'ils sont susceptibles de générer (Scott et Storper, 2003).

La reconnaissance du rôle de l'innovation, des connaissances et de l'apprentissage dans les processus de développement régional et territorial a eu des conséquences en termes d'évolution des politiques de développement, qui se caractérisent désormais le plus souvent par la combinaison d'interventions en faveur des infrastructures (transport, télécommunications haut débit...) et un soutien à des composantes moins tangibles comme la structuration de réseaux et le transfert de connaissances, afin de renforcer les capacités collectives de création de connaissances et d'apprentissage. L'enjeu est alors de construire des actifs endogènes au territoire, ce qui suppose la mobilisation des forces locales dans le cadre d'interactions où les logiques d'expérimentation (marquées par l'acceptation des processus d'essai-erreur) prennent le pas sur la mise en application de solutions prédéfinies, en dépit des contraintes pesant sur les finances publiques. C'est la raison pour laquelle de tels dispositifs d'intervention publique sont davantage liés au contexte de leur construction que dotés d'une valeur universelle. Toutefois, l'examen des stratégies engagées au niveau des territoires et des régions (de surcroit au sein d'un même cadre national) démontre la relativement faible créativité des solutions à l'œuvre et la difficulté pour la plupart des territoires de se démarquer nettement et durablement. 


\section{Vers une innovation territoriale ?}

En dépit d'avancées indéniables sur la compréhension des liens entre innovation, connaissance, apprentissage et développement régional au cours des vingt dernières années, les constructions théoriques ainsi proposées restent caractérisées par la diversité et la faiblesse de leur conceptualisation et de leur formalisation, ainsi que par le flou des messages adressés aux décideurs en vue d'améliorer les politiques publiques. Souvent fondées sur les seules activités high-tech, déterminées par la technologie et une culture d'entreprise répondant largement aux impératifs marchands, elles réduisent le champ de l'innovation aux dimensions les plus technologiques, négligeant au passage non seulement les innovations incrémentales mais aussi de nombreux territoires qui ne se distinguent pas par leur adhésion aux principes du high-tech mais se caractérisent néanmoins par une intense activité d'innovation de différentes sortes (sociale, organisationnelle, institutionnelle...). Par ailleurs, et au-delà d'une unité sémantique de façade sur les notions mobilisées, ces modèles analytiques sont en réalité porteurs de visions différentes des dynamiques d'innovation, qui nuisent à l'établissement d'un cadre théorique clair.

\section{Encadré 7.2. Un exemple d'innovation socio-territoriale : la transition agroécologique au Brésil}

\section{Marc Piraux et Philippe Bonnal}

La transition agroécologique de systèmes de production agricole est un processus complexe qui implique des changements techniques, sociaux et institutionnels. Le semi-aride brésilien, où la transition a été initiée il y a plus de 20 ans, constitue un exemple intéressant d'innovation. Partant du souci de résoudre des problèmes sociaux engendrés par le manque d'eau, aggravés par ailleurs par l'inadéquation des propositions techniques des pouvoirs publics et les méthodes interventionnistes d'un État relativement centralisé, la transition a conduit à une série de changements caractéristiques de l'innovation sociale et territoriale : i) ils répondent à la satisfaction des besoins des populations locales, une condition importante de l'innovation sociale ; ii) ils ont renforcé les processus d'apprentissage pour développer les capacités de lecture partagée des agroécosystèmes et valoriser les pratiques socio-productives peu ou pas valorisées socialement ; ces pratiques ont constitué le support des débats pour la construction d'un projet alternatif ; iii) ils ont pour base le mouvement social d'expérimentations menées par les agriculteurs eux-mêmes, ce qui a conduit à une consolidation des réseaux sociaux ; iv) ils valorisent enfin un processus de gouvernance multi-échelle des innovations agricoles qui favorise l'intégration sociale et l'insertion politique des paysans. Si ces innovations sont ancrées dans le territoire, elles ont permis aussi la création et la mise en place de dispositifs (tels que le réseau d'articulation du semi-aride - ASA) et de politiques publiques spécifiques à la région.

Ces innovations ont interpellé la capacité à faire évoluer la sphère publique liée aux actions de développement. L'expérimentation institutionnelle a donc été incontournable. L'innovation institutionnelle dont l'État a fait preuve consiste à avoir su transformer les expériences territorialisées d'acteurs sociaux en politiques publiques. En ce sens, elle peut être considérée comme l'appropriation par l'État d'une innovation 
développée à l'échelle locale. Le succès mitigé de cette transformation s'explique par le caractère périphérique et fragmentaire du changement institutionnel réalisé au sein de l'appareil d'État. Les trajectoires des innovations sociales et institutionnelles s'inscrivent dans des rapports de pouvoirs qu'elles cherchent à modifier. Il ressort que la durabilité des innovations socio-territoriales locales dépend aussi d'un mouvement complémentaire d'uniformisation des normes au sein même de l'État (Piraux et al., 2010 ; Bonnal et Piraux, 2010).

Une manière d'avancer sur ces questions, et en particulier d'intégrer la question de l'innovation à l'analyse de l'ensemble des territoires, y compris ruraux, serait d'élargir le débat à la prise en compte de la notion d'innovation territoriale, dans toutes ses dimensions. Son approfondissement doit permettre de progresser dans la compréhension de la déclinaison des progrès de l'humanité à l'échelle territoriale (Moulaert et Sekia, 2003) et de rendre l'analyse des modèles d'innovation utile pour les communautés locales. Certaines approches, par exemple les travaux du GREMI sur la notion de milieu innovateur (Camagni et Maillat, 2006), ont investigué la notion d'innovation territoriale dans des territoires plus ruraux ou moins développés, en s'appuyant sur les innovations organisationnelles et la mobilisation des populations locales. Les règles d'action collective et les dispositifs institutionnels sont alors considérés comme des facteurs explicatifs des dynamiques territoriales innovantes, et l'innovation regardée comme une construction sociale conditionnée par le contexte géographique dans lequel elle s'inscrit. Ancrée dans les pratiques, elle est donc nécessairement située dans l'espace. La question de l'innovation territoriale est également investie par les champs émergents de l'économie sociale et solidaire et du développement durable (Zaoual, 2008). Des concepts nouveaux ont été forgés comme celui d'innovation sociale (Klein et Harrison, 2007 ; Hillier et al., 2004) qui décrit un ensemble de pratiques d'innovation d'entreprises en réponse à des besoins sociaux peu ou non satisfaits et/ou mettant en ouvre des processus permettant d'inscrire une démarche de transformation sociale dans la durée. Ces initiatives révèlent la place éminente occupée par les territoires comme creuset des formes nouvelles d'organisation et de partenariat innovants, en milieu urbain comme dans les espaces ruraux.

\section{" Quelle gouvernance pour faire émerger l'innovation dans les espaces ruraux et périurbains?}

L'analyse des processus de développement, initialement centrée sur les dimensions économiques, s'est progressivement ouverte à la question de l'innovation, amenant à considérer le maillage des relations sociales et institutionnelles au niveau local, ainsi que les interactions et imbrications entre échelles et niveaux géographiques. Cette complexité accrue conduit à poser la question de la gouvernance des territoires, dans le but de faire émerger des processus innovants, mais aussi d'accorder 
les désirs des différentes composantes des populations locales et de les articuler avec les politiques et régulations globales.

Les processus de gouvernance des territoires sont aujourd'hui le lieu d'intenses bouleversements, qui contribuent aux phases d'innovation territoriale et constituent ainsi le moteur du développement et de la croissance dans les territoires ruraux ou urbains. Ces dispositifs de gouvernance sont en quelque sorte des laboratoires du changement, car ils permettent d'accompagner et parfois d'anticiper les mutations en cours dans les territoires, en leur donnant forme, en permettant de maintenir le dialogue et l'expression des oppositions, ainsi que d'éviter les affrontements violents, ou l'échec du développement par atonie ou expatriation. Ces changements s'incarnent sous les deux formes polaires et jumelles des conflits et de la concertation, qui constituent les modes d'expression et les courroies de transmission des innovations en cours au niveau des territoires.

\section{Concertation et négociation pour définir une vision partagée}

Il s'agit tout d'abord des mécanismes de négociation, et plus particulièrement de concertation, et de leur mise en place au niveau local. On peut, selon Beuret (2006), distinguer différents types d'opérations mobilisables au sein de démarches participatives, marquées par un niveau croissant d'implication, et qui contribuent à des niveaux divers aux processus de gouvernance des territoires. La communication cherche à faire passer un message et obtenir l'adhésion du public à une proposition : elle peut faire partie de démarches participatives, par exemple lorsqu'il s'agit de convaincre certains groupes qu'ils ont intérêt à participer plutôt qu'avoir recours au rapport de force ; l'information permet de transmettre des données qui permettront aux individus ou groupes cibles visés de se construire une opinion et de participer au débat ; la consultation vise à collecter les avis des acteurs, sans apporter de garanties quant à la prise en compte des opinions exprimées ; le dialogue va permettre des rapprochements entre les parties prenantes et la construction de langages et références communes; la concertation recherche la construction collective de visions, d'objectifs, de projets communs, en vue d'agir ou de décider ensemble ; enfin, la négociation cible l'obtention d'une décision acceptée par l'ensemble des parties prenantes.

Ces mécanismes ont donné naissance, ces dernières décennies, à des inventions et interventions de toutes natures, qui ont en commun de faciliter la mise en place du paradigme de la concertation, dont témoigne notamment le succès des travaux d'Ostrom $(1990 ; 2005)$ en matière de définition des dispositifs de gouvernance des ressources naturelles communes abordés par l'angle de la définition des droits de propriété par les communautés locales. Toutefois, ces mécanismes ne paraissent pas totalement stabilisés et génèrent de nombreux débats et controverses quant à leurs utilités respectives (Blatrix et al., 2007 ; Mermet et Berlan-Darqué, 2009). Un relatif consensus s'établit néanmoins pour admettre que la mise en œuvre de différentes formes de participation des acteurs privés ou semi-publics aux débats ou à la décision publique permet d'avancer vers des processus de gouvernance territoriale plus harmonieux et démocratiques. Il en résulte ainsi un certain nombre de mécanismes et d'outils de la gouvernance des territoires, comme celui qui est présenté 
dans l'encadré 7.3. En témoigne, dans le cas français, de la Loi Bouchardeau de 1983 à la Loi de 2002 relative à la démocratie de proximité, la complexification de la décision en matière de projets d'infrastructures publiques, avec la déclaration d'utilité publique, les enquêtes publiques, et l'instauration de la Commission nationale du débat public, des concertations préalables à l'élaboration et à la révision des documents d'urbanisme, puis des commissions consultatives des services publics locaux ${ }^{1} \ldots$

\section{Encadré 7.3. Co-construction d'un guide de la gouvernance territoriale Hélène Rey-Valette et Eduardo Chia}

Dans le cadre du projet Gouv.Innov ${ }^{1}$ (Gouvernance des territoires et développement rural : une analyse par les innovations organisationnelles), nous avons analysé les dynamiques de la gouvernance territoriale dans des situations et dispositifs de gestion intégrée et durable des territoires y compris périurbains. Une attention particulière a été portée à la place des acteurs agricoles. Il s'agissait d'observer, d'analyser et d'accompagner la mise en place et la conduite de dispositifs de gouvernance. Un objectif, opérationnel, était de proposer aux acteurs du développement local un guide pour la mise en œuvre de la gouvernance territoriale. Ce dernier a été co-construit par l'équipe pluridisciplinaire de chercheurs du projet Gouv.Innov et un panel d'agents de développement et de représentants du monde agricole. La co-construction du guide est partie d'une analyse générique de la gouvernance territoriale. Elle permet de tenir compte de la diversité des dispositifs ainsi que de leur caractère historiquement construit par rapport aux processus territoriaux ainsi que de leur encastrement spatial et des dynamiques multi-échelles. Quatre rubriques et étapes sont distinguées qui correspondent à des catégories complémentaires d'analyse, qui peuvent être déroulées de façon séquentielle ou en se limitant à un des aspects selon les besoins :

- améliorer la compréhension (étape 1, connaissance des acteurs, institutions et procédures) ;

- analyser (approches détaillées des interactions fonctionnelles (étape 2) et territoriales (étape 3) nécessitant des enquêtes, des entretiens ou des formes d'observation participante);

- évaluer les produits et les effets du processus (étape 4), correspond au niveau le plus approfondi qui intègre une logique de réflexivité avec des besoins d'information plus importants.

Le guide issu de ce travail collectif de co-construction témoigne du caractère opératoire de la définition opérationnelle de la gouvernance en offrant des voies pour renouveler l'ingénierie de la gouvernance territoriale dans plusieurs champs, en renforçant : (i) les phases préparatoires, (ii) l'appui aux acteurs, (iii) le pilotage et la coordination de l'action publique, (iv) l'évaluation et (v) l'institutionnalisation. Il offre aussi plusieurs illustrations de terrain. Il constitue un « outil » de réflexivité et d'accompagnement qui contribue à l'ingénierie de la gouvernance territoriale http:/www.lameta.univ-montp1.fr/ggov (Rey-Valette et al., 2010, 2011).

1. Projet financé par le programme «Pour et Sur le Développement Régional (PSDR)» du LanguedocRoussillon. 
Les processus de concertation, marqués par une intention coopérative, constituent l'un des laboratoires les plus importants de la coordination en vue de la gouvernance des territoires. Basée sur une mise en relation structurée et durable entre des acteurs qui acceptent de partager de l'information, de discuter de problèmes ou d'enjeux spécifiques afin de convenir d'objectifs communs et d'éventuelles actions collectives (Bourque, 2008), leur construction collective permet de les distinguer des autres formes de coopération et de participation à l'action publique. La concertation recouvre ainsi « des processus de construction collective de visions, d'objectifs, de projets communs, en vue d'agir ou de décider ensemble » (Beuret, 2006), mais peut aussi être utilisée par un acteur qui entend se positionner comme tiers, pour favoriser une coordination entre diverses parties, comme c'est le cas pour les agents de développement par exemple. Elle prend forme sur des scènes, ou arènes, autour desquelles s'articulent les échanges entre des groupes composés de personnes physiques ou morales caractérisées par les mêmes actes relatifs à l'objet de la concertation et les mêmes comportements et prises de positions. L'histoire du processus de concertation est souvent faite d'une ou plusieurs controverses, mais le scénario n'est pas écrit à l'avance et sera élaboré au fil d'un itinéraire de concertation.

\section{Le rôle des conflits dans les processus d'innovation}

Nos recherches sur les conflits dans les espaces ruraux et périurbains (Torre et al., 2006, 2010) révèlent que cette dimension est également essentielle dans les processus d'aménagement du territoire, de développement régional ou de gouvernance des diverses fonctionnalités locales; elle s'impose, en termes de recours aux tribunaux, de manifestations médiatiques ou de démonstration de violence. Loin d'être, la plupart du temps, des oppositions aveugles ou purement égoïstes, les conflits d'usage de l'espace constituent ainsi une manière d'entrer dans la discussion sur les enjeux et les chemins du développement territorial, et d'infléchir les décisions en prenant part au processus en cours alors que l'on en avait été exclu (Dowding et al., 2000). C'est la raison pour laquelle ils portent, soit sur les décisions qui ont été prises en matière d'aménagement (négociation arbitrée), soit sur la composition et la représentativité des instances en charge de la décision (négociation arbitrale). Le conflit fait ainsi partie intégrante du processus de délibération au niveau local, en permettant une expression de la démocratie locale, ainsi que la réintégration de parties prenantes oubliées ou lésées dans une phase antérieure d'élaboration des projets.

Les conflits d'usage de l'espace constituent ainsi une forme de résistance et d'expression des oppositions à des décisions qui laissent insatisfaite une partie de la population locale (Darly et Torre, 2011). Certaines innovations locales, qu'elles soient de nature technique ou organisationnelle, provoquent une résistance, qui peut donner naissance à des conflits. Les changements majeurs, qui impliquent une reconfiguration des usages de l'espace (installation d'infrastructures de transport ou de déchets, nouveaux plans locaux d'urbanismes, zonages territoriaux ou environnementaux) génèrent des conflits dont l'étendue spatiale et sociale peut prendre beaucoup d'ampleur. Les conflits sont des signaux des évolutions sociales, techniques et économiques, des révélateurs de la nouveauté et des innovations. Ils témoignent des oppositions que suscitent ces dernières, des discussions autour de leur mise en 
œuvre, de leur (non) acceptabilité éventuelle, ainsi que de la mise en place des procédures de gouvernance et de leur transformation sous l'influence de ces dynamiques de changement. Tout changement provoque des oppositions ou des résistances, plus ou moins fondées ou pertinentes. Il serait toutefois simpliste de considérer ces résistances comme le signe systématique d'une opposition réactionnaire au changement. Dans de nombreux cas, elles reflètent davantage des divergences quant aux orientations soutenues par les initiatives nouvelles qui s'imposent aux populations, qu'une volonté de maintien immuable des situations antérieures. Pendant ces phases de conflit se jouent des recompositions sociales ou de groupes d'intérêts, et des changements de nature technique ou juridique. Après le conflit restent les nouveaux accords au niveau local, les nouveaux modes de gouvernance, les nouvelles configurations des tours de tables, ainsi que les actes techniques (changements de tracés, aménagements divers, modifications des documents d'urbanisme...), qui résultent des négociations précédentes. Ferments de l'innovation territoriale, les conflits sont ainsi à la fois issus et à l'origine des mutations territoriales.

La gouvernance des territoires ne se limite donc pas à une vision idyllique des relations économiques et sociales, i.e. aux formes de coopération et de constructions communes. Il s'agit également d'une interaction entre des forces poussant à la coopération et d'autres forces, qui poussent au conflit (Torre et Traversac, 2011). Loin d'être un long fleuve tranquille les processus de développement territorial et leur déroulement dans le temps sont faits de phases de négociations, de collaboration ou d'apaisement, mais également de périodes beaucoup plus animées, ou conflictuelles, au cours desquelles certains groupes ou catégories d'acteurs s'opposent, parfois avec violence, pour définir les marches à suivre et les options à retenir. Le processus de gouvernance des territoires présente ainsi deux faces complémentaires, dont l'importance réciproque varie selon les périodes et les situations. Il se nourrit de ces tendances opposées (Glazer et Konrad, 2005), dont la synthèse conduit à la définition de sentiers de développement territorial.

\section{W Conclusion}

Aujourd'hui de nombreux auteurs considèrent que se dessine un nouveau paradigme du développement rural, qui s'autonomiserait du modèle agro-industriel et hygiénique de production dominant fondé sur l'utilisation d'intrants chimiques et le contrôle sanitaire des produits, tout en construisant une représentation des espaces ruraux alternative à celle de la dépendance à l'égard du phénomène d'urbanisation (Röling et de Jong, 1998 ; Marsden, 2006). S'y ajoute la montée des problématiques environnementales et de développement durable, qui viennent à la fois impacter fortement la conception des activités présentes sur les zones rurales - et tout particulièrement l'activité agricole - et infléchir les politiques publiques et leurs déclinaisons locales, en particulier en matière de zonages (Natura 2000, directives habitats, trames vertes et bleues, etc.).

Ce nouveau paradigme émergerait à la fois dans les pratiques et interventions des acteurs de terrain et dans les politiques publiques, le développement rural se pensant alors comme un processus multi-niveaux, multi-acteurs et multi-facettes (van der 
Ploeg et al., 2000). Multi-niveaux en matière de diversité des politiques et institutions destinées à traiter la question du développement rural, ainsi que d'évolution des relations entre agriculture et société, avec la prise en compte de la production de biens publics, la construction d'un nouveau modèle de production agricole intégrant les interactions entre agriculture et autres activités, et la combinaison des activités à l'échelle des entreprises en milieu rural. Multi-acteurs, avec les interactions entre agriculteurs et autres acteurs des espaces ruraux et les politiques de développement rural visant à faire émerger de nouvelles articulations entre local et global, mais pouvant également être un moyen de restaurer la légitimité des élites locales ou de jouer sur les intérêts clientélistes. Multi-facettes enfin : le développement rural se déploie en une gamme de pratiques différenciées dont certaines sont émergentes et parfois interconnectées (gestion des paysages, conservation de la nature, agritourisme, agriculture biologique, produits agricoles spécifiques, circuits courts...), de sorte que des éléments considérés comme superflus dans le paradigme moderniste acquièrent des rôles nouveaux dans les relations entre exploitations, mais aussi entre agriculteurs et population urbaine.

\section{" Références bibliographiques}

Amable B., Barré R., Boyer R., 1997. Les systèmes d'innovation à l'ère de la globalisation, Economica, Paris.

Autant-Bernard C., Mairesse J., Massard N., 2007. Spatial knowledge diffusion through collaborative networks. Papers in Regional Science, 86 (3), 341-350.

Becattini G., 1990. The Marshallian Industrial Districts as a Socio-Economic Notion, In : Industrial Districts and Inter-Firm Cooperation in Italy (Pyke F., Becattini G., Sengenberger W., eds), International Institute of Labour Studies, Geneva.

Beuret J.E., 2006. La conduite de la concertation. Pour la gestion de l'environnement et le partage des ressources, L'Harmattan, Paris, $340 \mathrm{p}$.

Blatrix C., Blondiaux L., Fourniau J.M., Heriad-Dubreil B., Lefebvre R., Revel M., 2007. Le débat public : une expérience française de démocratie participative. La Découverte, $416 \mathrm{p}$.

Bonaudo T., Coutinho C., Poccard-Chapuis R., Lescoat P., Lossouarn J., Tourrand J.-F., 2010. Poultry industry and the sustainable development of territories: what links? what conditions? In : Innovation and Sustainable Development in Agriculture and Food (ISDA), Montpellier, France http://hal.archives-ouvertes.fr/hal-00522800/fr/

Bonnal P., Piraux M., 2010. Actions publiques territoriales en milieu rural et innovations l'exemple du territoire de la Borborema et de l'articulation du semi-aride au Brésil, In : Innovation and Sustainable Development in Agriculture and Food (ISDA), Montpellier, France - http://hal.archivesouvertes.fr/hal-00522109/fr/

Boschma R., Frenken K., 2011. The emerging empirics of evolutionary economic geography. Papers in Evolutionary Economic Geography, 11.01, URRC, Utrecht University.

Boschma R., 2009. Evolutionary economic geography and its implications for regional innovation policy. Papers in Evolutionary Economic Geography, 09.12, URRC, Utrecht University.

Bourque D., 2008. Concertation et partenariat. Entre levier et piège du développement des communautés, Presses de l'Université du Québec, Collection Initiatives, 142 p.

Camagni R., Maillat D., 2006. Milieux Innovateurs. Théorie et politiques. Anthropos, Economica, Paris.

Camagni R., 1995. The concept of innovative milieu and its relevance for public policies in European lagging regions. Papers in Regional Science, 74 (4), 317-340.

Cohendet P., Simon L., 2008. Knowledge intensive firms, communities and creative cities, Oxford University Press. 
Colletis G., Pecqueur B., Rychen F., Zimmermann J., et al., 1999. Construction territoriale et dynamiques productives. Sciences de la Société, 48, 35-54.

Cooke P., Morgan K., 1998. The Associational Economy: Firms, Regions and Innovation, Oxford University Press, Oxford.

Darly S., Torre A., 2011. Land-use conflicts and the sharing of resources between urban and agricultural activities in the Greater Paris Region. Results based on information provided by the daily regional press, In : Towns in a rural world (de Noronha Vaz T., Van Leeuwen E., Nijkamp P., eds), Ashgate, London.

Davezies L., 2008. La République et ses Territoires. Editions du Seuil, Paris.

Dowding K., John P., Mergoupis T., Van Vugt M., 2000. Exit, voice and loyalty: Analytic and empirical developments. European Journal of Political Research, 37, 469-495.

Dunford M., 1993. Regional disparities in European Community: evidence from the REGIO databank. Regional Studies, 27 (8), 727-743.

Feldman M., 2000. Location and innovation: the new economic geography of innovation, spillovers and agglomeration, In: The Oxford Handbook of Economic Geography (Clark G.L., Feldman M., Gertler G., eds), Oxford University Press, Oxford.

Freeman C., 1995. The 'National System of Innovation' in Historical Perspective. Cambridge Journal of Economics, 19, 5-24.

Frenken K., Boschma R., 2007. A theoretical framework for evolutionary economic geography: industrial dynamics and urban growth as a branching process. Journal of Economic Geography, 7 (5), 635-649.

Fujita M., Thisse J.F., 1997. Economie géographique, problèmes anciens et nouvelles perspectives. Annales d'Economie et de Statistique, 45, 37-87.

Fujita M., Thisse J.F., 2001. Economie et marché. Cahiers d'Economie et de Sociologie Rurale, 58-59, 11-57.

Glazer A., Konrad K.A., 2005. Conflict and Governance. Springer Verlag, 201 p.

Goldstein H., 2009. Theory and Practice of Technology-based Economic Development, In: Theories of Local Economic development (Rowe J.E., ed.), Ashgate, Burlington, 375 p.

Hall P., 1994. Innovation, Economics and Evolution. Harvester Wheatsheaf, New York.

Hillier J., Moulaert F., Nussbaumer J., 2004. Trois essais sur le rôle de l'innovation sociale dans le développement territorial. Géographie, économie, société, 2/2004 (6), 129-152.

Johansson B., Karlsson C., Stough R.R., 2001. Theories of Endogenous Regional Growth, Springer, Heidelberg, New York.

Klein J.L., Harrison D., 2007. L'innovation sociale. Emergence et effets sur la transformation des sociétés, Presses de l'Université du Québec.

Krugman P., 1991. Geography and Trade, MIT Press, Cambridge.

Lundvall B.-A., 1992. National Innovation System: Towards a Theory of Innovation and Interactive Learning, Pinter, London

Lundvall B.-A., Maskell P., 2000. Nation states and economic development: from national systems of production to national systems of knowledge creation and learning, In : The Oxford Handbook of Economic Geography (Clark G.L., Feldman M., Gertler G., eds), Oxford University Press, Oxford.

Malecki E., 1997. Technology and Economic Development: The Dynamics of Local, Regional and National Competitiveness, 2nd ed., Addison Wesley Longmann, London.

Markusen A., 1996. Sticky Places in Slippery Space: a Typology of Industrial Districts. Economic Geography, 72 (2), 294-314.

Marsden T., 2006. Pathways in the sociology of rural knowledge, In : The Handbook of Rural Studies (Cloke P., Marsden T., Mooney P., eds), Sage Publications, London, 510 p.

Maskell P., 2001. The firm in economic geography. Economic Geography, 77 (4), 329-344.

Mermet L., Berlan-Darqué M., 2009. Environnement : décider autrement. Nouvelles pratiques et nouveaux enjeux de la concertation, L'Harmattan, $378 \mathrm{p}$.

Moulaert F., Sekia F., 2003. Territorial Innovation Models: A Critical Survey. Regional Studies, 37 (3), 289-302. 
Muchnik J., de Sainte Marie C., 2010. Le temps des Syal. Techniques, vivres et territoires, éditions Quae, Versailles.

Muchnik J., 1996. Systèmes agroalimentaires localisés : organisation, innovations et développement local, Proposition issue de la consultation du Cirad Stratégies de recherche dans le domaine de la socioéconomie de l'alimentation et des industries agroalimentaires, Cirad, $27 \mathrm{p}$.

Nelson R., 1993. National Innovations Systems. A Comparative Analysis, Oxford University Press, Oxford.

Nooteboom, B., 2000. Learning and Innovation in Organizations and Economies. Oxford University Press, Oxford.

Ostrom E., 1990. Governing the commons: The evolution of institutions for collective action, Cambridge University Press.

Ostrom E., 2005. Understanding Institutional Diversity, Princeton University Press, Princeton, NJ.

Ottaviano G., Thisse J.F., 2004. Agglomeration and economic geography, In : Handbook of Regional and Urban Economics (Henderson, J.V., Thisse, J.F., eds), edition 1, Elsevier, vol. 4, chap. 58, 2563-2608.

Perrier-Cornet P., 2009. Les systèmes agroalimentaires localisés sont-ils ancrés localement ? Un bilan de la littérature contemporaine sur les Syal, In : Politiques agricoles et territoires (Aubert F., Piveteau V., Schmitt B., eds), éditions Quae, 223 p.

Perroux F., 1961. L'économie du xx ${ }^{\mathrm{e}}$ siècle. Presses Universitaires de Grenoble, Grenoble.

Piraux M., Silveira L., Diniz P., Duque G., 2010. La transition agroécologique comme une innovation socio-territoriale. In : Innovation and Sustainable Development in Agriculture and Food (ISDA), Montpellier, France - http://hal.archives-ouvertes.fr/hal-00512788/fr/

Porter M.E., 1985. Competitive Advantage, The Free Press, New York.

Porter M.E., 1990. The Competitive Advantage of Nations, The Free Press, New York.

Puttilli M., Tecco N., 2010. Implications of biodiesel production chains in restructuring rural space, In : Innovation and Sustainable Development in Agriculture and Food (ISDA), Montpellier, France - http://hal.archives-ouvertes.fr/hal-00520767/fr/

Regional Science Policy and Practice, 2011. Special issue on innovation and creativity as the core of regional and local development policy, August.

Rey-Valette H., Chia E., Soulard C., Mathe S., Michel L., Nougaredes B., et al., 2010. Innovations et gouvernance territoriale : une analyse par les dispositifs, In : Innovation and Sustainable Development in Agriculture and Food (ISDA), Montpellier, France - http://hal.archives-ouvertes.fr/hal00520264/fr/

Röling N., de Jong F., 1998. Learning: Shifting paradigms in education and extension studies. The Journal of Agricultural Education and Extension, 5 (3), 143-161.

Sanz Cañada J., 2010. Territorial externalities in local agro-food systems of typical food products the olive oil protected designations of origin in Spain. In : Innovation and Sustainable Development in Agriculture and Food (ISDA), Montpellier, France - http://hal.archives-ouvertes.fr/hal-00530963/ $\mathrm{fr} /$

Scott A.J., Storper M., 2003. Regions, globalization, development. Regional Studies, 37 (6-7), 579593.

Solow R.M., 2000. Growth theory: an exposition, Oxford University Press, New York.

Stöhr W.B., 1986. Regional Innovation Complexes. Papers in Regional Science, 59, 29-44.

Torre A., Traversac J.B., 2011. Territorial Governance, Springer Verlag, Heidelberg and New York.

Torre A., Wallet F., 2012. The intriguing question of regional and territorial development in rural areas. Analytical variations and public policies. European Planning Studies, Special issue on Rural areas and Agrofood business (accepted).

Torre A., 2008. On the role played by temporary geographical proximity in knowledge transfer. Regional Studies, 42 (6), 869-889.

Torre A., 2010. Conflits environnementaux et territoires, In : Développement Durable et Territoire (Zuindeau B., ed.), Presses Universitaires du Septentrion, 518 p. 
Torre A., Aznar O., Bonin M., Caron A., Chia E., Galman M., Guérin M., Jeanneaux P., Kirat T., Lefranc C., Melot R., Paoli J.C., Salazar M.I., Thinon P., 2006. Conflits et tensions autour des usages de l'espace dans les territoires ruraux et périurbains. Le cas de six zones géographiques françaises. Revue d'Economie Régionale et Urbaine, 3, 415-453.

van der Ploeg J.D., Renting H., Brunori G., Knicken K., Mannion J., Marsden T., de Roest K., Sevilla Guzman E., Ventura F., 2000. Rural Development: from practices and policies towards theory. Sociologia Ruralis, 40 (4), 391-408.

Wolfe D.A., Gertler M., 2002. Innovation and social learning: an introduction, In : Innovation and Social Learning: Institutional Adaptation in an Era of Technological Change (Gertler M., Wolfe D.A., eds), Palgrave Macmillan, Basingstoke.

Zaoual H., 2008. Développement durable des territoires : économie sociale, environnement et innovations, Coll. marchés et organisations, L'Harmattan. 
Partie 3

Concevoir des futurs pour l'agriculture et l'agroalimentaire 



\title{
Chapitre 8 \\ L'agrobiodiversité, vers des instruments de protection innovants
}

\author{
Juliana SANTILLI
}

\begin{abstract}
À l'intersection de la biodiversité et de l'agriculture, le concept innovant d' « agrobiodiversité » a émergé au cours des 10 à 15 dernières années, dans un contexte interdisciplinaire faisant intervenir plusieurs domaines de savoir (agronomie, anthropologie, écologie, botanique, génétique, biologie de la conservation, etc.). Il reflète les relations dynamiques et complexes qui existent entre les sociétés humaines, les plantes cultivées, les animaux domestiques et les écosystèmes au sein desquels tous interagissent. L'agrobiodiversité entretient des liens directs avec la sécurité alimentaire, la santé, l'équité sociale, la lutte contre la faim, la durabilité environnementale et le développement durable rural. Tout comme la biodiversité sauvage, l'agrobiodiversité a été perçue comme menacée et nécessitant impérieusement d'être sauvegardée par le biais de nouveaux instruments juridiques tant au niveau national qu'international. Après une exposition du raisonnement qui soustend la notion d'agrobiodiversité, ce chapitre présente la gamme des nouveaux outils juridiques qui s'y appliquent et qui tendent à adopter un point de vue plus systémique de l'agroécosystème, considéré comme un tout, au lieu de se concentrer sur des constituants particuliers. Ces nouveaux instruments juridiques prêtent pour la première fois attention aux droits des agriculteurs, aux systèmes agricoles en tant qu'éléments du patrimoine culturel, et au rôle des aires protégées dans la promotion de l'agrobiodiversité. La sauvegarde des systèmes ingénieux du patrimoine agricole mondial est également abordée. Les deux dernières décennies ont vu chez les législateurs s'engager un processus d'innovation fertile qui vise à protéger et à valoriser les systèmes agricoles locaux traditionnels et l'agrobiodiversité élevée qu'ils recèlent.
\end{abstract}

\section{" L'agrobiodiversité : un concept en construction}

La biodiversité, ou diversité biologique, c'est-à-dire la diversité des formes de vie, recouvre trois niveaux de variabilité : la diversité des espèces, la diversité génétique (la variabilité qui existe au sein d'un ensemble d'individus appartenant à une même espèce) et la diversité écologique, qui prend en compte les différents écosystèmes et paysages. Il en va de même avec l'agrobiodiversité, qui intègre la diversité des espèces (celle des différentes espèces cultivées, telles que le riz, le maïs, la courge, 
la tomate, etc., appelée diversité interspécifique), la diversité génétique au sein de chaque espèce (celle des différentes variétés de maïs, de haricot, etc., appelée diversité intraspécifique), et la diversité des écosystèmes cultivés, également désignés sous le terme d'agroécosystèmes (systèmes agroforestiers, agriculture itinérante, jardins potagers, rizières, etc.). Les connaissances et la culture locales font également partie intégrante de l'agrobiodiversité, dans la mesure où cette dernière est entretenue par une activité humaine, l'agriculture.

Selon Cromwell et al. (2003), la biodiversité agricole englobe :

- des végétaux supérieurs, à savoir les plantes cultivées, les plantes sauvages récoltées et gérées pour l'alimentation, les arbres sur les terres agricoles, pâturages et parcours ;

- des animaux supérieurs, à savoir, les animaux domestiques, les animaux sauvages chassés pour l'alimentation, ainsi que les poissons sauvages et d'élevage ;

- des arthropodes, essentiellement des insectes, y compris des pollinisateurs (abeilles, papillons, par exemple), les insectes nuisibles (guêpes, coléoptères, par exemple) et les insectes participant au cycle du sol (notamment les termites) ;

- d'autres macro-organismes (par exemple les vers de terre);

- des micro-organismes (par exemple rhizobia, champignons, pathogènes produisant des maladies).

Les fonctions de l'agrobiodiversité sont liées à la production durable de produits agricoles et d'autres produits alimentaires, y compris la fourniture de composants de base pour l'évolution ou l'amélioration génétique délibérée de nouvelles variétés végétales utiles; le soutien biologique de la production par le biais, par exemple, du biotope du sol, des pollinisateurs et des prédateurs; et un plus large éventail de services écologiques assurés par les agroécosystèmes, tels que la protection du paysage, la préservation des sols, la santé, le cycle et la qualité de l'eau, et la qualité de l'air (Cromwell et al., 2003).

On associe le plus souvent l'agrobiodiversité aux plantes cultivées. Toutefois, Cromwell et al. (2003) font remarquer que les espèces de plantes sauvages jouent un rôle important sur le plan nutritionnel et culturel. Les aliments tirés d'espèces sauvages font partie intégrante du régime alimentaire quotidien de nombreux ménages pauvres en milieu rural. En outre, la diversité des animaux domestiques constitue une autre grande composante de l'agrobiodiversité. Non seulement ils fournissent des aliments, mais aussi des vêtements, engrais et combustibles (tirés du fumier). Les forces sociales et culturelles sont souvent les principaux facteurs à l'œuvre dans la diversification des animaux domestiques (et des systèmes de production associés) et dans le façonnage des races typiques d'une localité. La plupart des races locales des zones rurales ont été créées par des communautés d'éleveurssélectionneurs, et la gestion efficace de la diversité génétique animale est déterminante pour la sécurité alimentaire et pour le développement durable à l'échelle mondiale.

La Convention sur la diversité biologique (CDB) ne propose aucune définition de l'agrobiodiversité, mais, selon la décision V/5 adoptée au cours de la Ve Conférence des parties à la CDB (CDP-5) : «L'expression diversité biologique agricole inclut toutes les composantes de la diversité biologique relatives à l'alimentation et l'agriculture : l'hétérogénéité et la variabilité des végétaux, des animaux et des 
micro-organismes au niveau génétique, des espèces et des écosystèmes qui sont nécessaires pour préserver les fonctions clés de l'agro-écosystème, de ses structures et de ses processus ». Ainsi, selon la décision V/5 de la CDB, la Conférence des parties a reconnu « la nature particulière de la diversité biologique agricole, ses caractéristiques, et les problèmes exigeant des solutions distinctives ». Les caractères distinctifs comprennent les points suivants :

- la diversité biologique agricole est essentielle pour répondre aux besoins alimentaires fondamentaux des humains et assurer leur subsistance ;

- la diversité biologique agricole est gérée activement par les agriculteurs; plusieurs éléments constitutifs de la diversité biologique agricole ne pourraient survivre sans cette intervention humaine. Le savoir et la culture autochtones font partie intégrante de la gestion de la diversité biologique agricole ;

- il y a une grande interdépendance entre pays à l'égard des ressources génétiques pour l'agriculture et l'alimentation, surtout parce plusieurs importants systèmes agricoles dépendent de cultures et d'espèces animales introduites de l'extérieur ;

- pour les cultures et les animaux domestiques, la diversité à l'intérieur des espèces est au moins aussi importante que la diversité entre les espèces, et elle a été largement accrue par l'agriculture ;

- à cause de l'importance de l'intervention humaine dans la gestion de la diversité biologique agricole, sa conservation dans des systèmes de production est intrinsèquement liée à l'utilisation durable. La préservation au moyen de zones protégées est moins importante ;

- néanmoins, dans les systèmes agricoles de type industriel, la diversité biologique est largement conservée ex situ dans des banques de gènes ou des produits pour l'élevage et non pas à la ferme ;

- les interactions entre l'environnement, les ressources génétiques et les modes de gestion qui se produisent in situ au sein des agrosystèmes contribuent souvent au maintien d'un ensemble dynamique d'éléments constitutifs de la diversité biologique agricole

\section{H Les diverses contributions de l'agrobiodiversité}

C'est la diversité des plantes cultivées et des animaux domestiques, et leur capacité à s'adapter aux conditions adverses de l'environnement (climat, sol, végétation, etc.) ainsi qu'aux besoins spécifiques des êtres humains, qui permet aux agriculteurs de survivre dans les régions les plus difficiles de la planète. Dans bien des circonstances, c'est le fait de cultiver plusieurs espèces et variétés différentes qui protège le paysan contre une perte totale de sa récolte due à des ravageurs, des maladies, des sécheresses, des inondations, ou d'autres facteurs. Par contre, dans les monocultures, dont la base génétique est extrêmement étroite, c'est l'inverse qui est vrai : les ravageurs et les maladies s'attaquent à la seule espèce cultivée et anéantissent l'ensemble de la récolte.

L'agrobiodiversité est par ailleurs un outil essentiel pour progresser vers une agriculture plus durable. L'agriculture est considérée durable lorsque les activités agricoles produisent des denrées alimentaires et d'autres produits pour satisfaire les 
besoins humains de manière pérenne, sans incidence dégradante à long terme sur l'environnement. Pour y parvenir, l'agriculture doit éviter d'infliger des dommages graves ou irréversibles aux services des écosystèmes dont elle dépend (tels que la fertilité des sols, la quantité et la qualité de l'eau, la variabilité génétique, les pollinisateurs, etc.) et doit avoir un impact acceptable sur l'environnement plus général (gestion environnementale responsable). Au lieu de s'en remettre aux intrants extérieurs, l'agriculture durable s'appuiera de plus en plus sur la biodiversité agricole, utilisant au mieux une gamme aussi large que possible d'espèces et de variétés pour augmenter la résilience du système face aux chocs extérieurs et pour accroître les récoltes (Bioversity International, 2010).

L'agrobiodiversité n'est pas seulement associée à la production durable de produits alimentaires. Elle contribue également de manière importante à favoriser la santé et la sécurité alimentaire et nutritionnelle des populations humaines. Les nutritionnistes recommandent un régime alimentaire diversifié (équilibré en protéines, vitamines, minéraux et autres nutriments), condition fondamentale pour rester en bonne santé. Un régime alimentaire déséquilibré peut entraîner une « faim invisible » due à des carences en micronutriments essentiels (vitamines et oligo-éléments) dans l'alimentation quotidienne. Les effets de cette « faim invisible », très divers, peuvent aller jusqu'à des retards du développement mental, un affaiblissement du système immunitaire et une perte de force et d'énergie. Environ 2 milliards de personnes seraient ainsi concernées par une carence en fer, et autour de 800 millions manqueraient de vitamine A, dont la carence est susceptible d'entraîner une cécité dans les cas graves (GTZ, 2006). Le meilleur moyen de se prémunir contre les carences alimentaires est d'avoir un régime alimentaire varié, ce qui permet de veiller à un apport suffisant de tous les macro-éléments et micro-éléments indispensables à une bonne santé.

Seuls des systèmes agricoles diversifiés sont en mesure de proposer une alimentation plus nutritive et mieux équilibrée. La lutte contre la faim et la pauvreté passe nécessairement par des pratiques agricoles plus durables. Il existe une relation directe entre la diminution de la diversité agricole et l'appauvrissement des régimes alimentaires. L'érosion génétique dans les champs n'affecte pas seulement les agriculteurs, mais également les consommateurs. Les modèles de productions agricoles ont des répercussions directes sur l'alimentation et la santé des êtres humains. L'agriculture « moderne » et l'utilisation d'un nombre d'espèces réduit favorise une standardisation des habitudes alimentaires et une perte de l'intérêt culturel pour les espèces locales. L'agrobiodiversité est ainsi directement associée à au moins deux des objectifs du millénaire pour le développement : l'éradication de la pauvreté et de la faim, et la durabilité environnementale. Selon la FAO (Sommet mondial de l'alimentation, 1996), la sécurité alimentaire est assurée quand « toutes les personnes, en tout temps, ont économiquement, socialement et physiquement accès à une alimentation suffisante, sûre et nutritive qui satisfait leurs besoins nutritionnels et leurs préférences alimentaires pour leur permettre de mener une vie active et saine ».

Enfin, il est important de souligner que l'agrobiodiversité est une composante essentielle des stratégies nationales et internationales d'adaptation au changement climatique planétaire. Il existe une relation étroite entre le changement climatique, la sécurité alimentaire et l'agrobiodiversité : c'est grâce à la diversité qu'ils recèlent que les espèces, les variétés et les agroécosystèmes sont capables de s'adapter aux 
changements et aux variations des conditions du milieu. Ils ne sauront surmonter les défis futurs, dont ceux amenés par le changement climatique, que si la diversité génétique qu'ils ont en eux est suffisamment grande. Les interactions entre l'agrobiodiversité et le changement climatique sont nombreuses - ce dernier a déjà un effet, en réduisant le nombre des espèces et des écosystèmes agricoles. L'agrobiodiversité, quant à elle, est indispensable pour pouvoir faire face aux conséquences du réchauffement planétaire.

\section{W L'agrobiodiversité et les instruments juridiques}

\section{L'agrobiodiversité et les législations sur les semences}

Les semences - un terme qui sera utilisé ici dans son acceptation la plus large, incluant l'ensemble du matériel de propagation de végétaux - contiennent toute la vie d'une plante et constituent la base même de l'agrobiodiversité. On ne peut de ce fait bien comprendre l'impact des systèmes juridiques sur la diversité agricole sans analyser les réglementations qui régissent la production, la commercialisation et l'utilisation des semences, collectivement appelées législation sur les semences.

Les législations sur les semences ont pour objectif de veiller à l'identité et à la qualité du matériel de propagation végétal, en réglementant la manière avec laquelle ce dernier est produit, utilisé et commercialisé. Elles doivent être distinguées des droits de la propriété intellectuelle, ainsi que des droits des obtenteurs, qui confèrent des droits de propriété sur les nouvelles variétés végétales. Cependant, il reste que les législations sur les semences et les droits de propriété intellectuelle sur les variétés végétales suivent une logique semblable et ont certains concepts en commun.

Les lois sur les semences ont été adoptées en tant que «support juridique » au processus de « modernisation » et d' « industrialisation » de l'agriculture, dont les principaux paradigmes étaient le productivisme, la standardisation des produits agricoles et la segmentation des différentes étapes qui interviennent dans la production agricole. Ce nouveau paradigme industriel a favorisé les variétés végétales à haut rendement, homogènes et stables, très dépendantes de l'apport d'intrants de provenance extérieure. Dans son analyse de l'émergence et de l'évolution des systèmes semenciers formels et commerciaux, Louwaars (2007) explique qu'ils ont vu le jour dans les pays industrialisés au cours de la deuxième moitié du XIX ${ }^{\mathrm{e}}$ siècle. Dans les années 1960 et 1970, beaucoup de pays en voie de développement ont commencé à leur tour à promouvoir ces systèmes, avec l'appui des organisations internationales. Cependant, les systèmes semenciers formels et commerciaux n'ont jamais été en mesure de remplacer entièrement les systèmes semenciers informels des agriculteurs eux-mêmes et leur matériel végétal bien adapté aux conditions locales.

Bonneuil et al. (2006) expliquent que la «modernisation » de l'agriculture a encouragé l'idée que l'amélioration des plantes et la production des semences étaient des activités à réserver à des secteurs professionnels spécialisés (sélectionneurs, agronomes, etc.). Cette conception considérait les agriculteurs uniquement comme des producteurs, simples consommateurs de semences et d'intrants agricoles produits de 
manière industrielle, et leur déniait le rôle d'innovateurs et de détenteurs de savoirs et de savoir-faire d'importance cruciale pour la durabilité des agroécosystèmes et pour la conservation de l'agrobiodiversité in situ, et sur les exploitations mêmes (on farm). Les semences et les variétés produites par les agriculteurs, adaptées aux conditions locales, ont été de plus en plus remplacées par des variétés « modernes » uniformes. Comme l'expliquent Bonneuil et al. (2006), ces conceptions - qui stipulent que les variétés homogènes et stables sont ce qu'il y a de mieux pour les systèmes agricoles, quels qu'ils soient, et que seuls des scientifiques professionnels

\section{Encadré 8.1. Quand des paysans de communautés pauvres combinent sélection variétale participative et gestion in situ et $e x$ situ (Honduras) \\ Henri Hocdé, Juan Carlos Rosas et Rodolfo Araya}

Dans les zones montagneuses du centre du pays, perchées à $1000 \mathrm{~m}$ d'altitude, des communautés pauvres qui ne bénéficient pas ou peu des services de la recherche (concentrés sur des zones géographiques plus propices à des conditions de production dites « modernes ») s'organisent en Comité de recherche agricole local (dénommé CIAL). Avec l'appui de professionnels d'ONG et de quelques chercheurs sélectionneurs nationaux, ils se lancent dans la création variétale de cultures vivrières (haricot et maïs). Avec succès, au terme de nombreuses années qui ont exigé patience, ténacité, persévérance, apprentissage, doutes, motivation, organisation. Palmichal, Cedron, Nueva Esperanza, Capulin, Olotillo mejorado... de nouvelles variétés apparaissent dans le paysage et sont cultivées. Concomitamment, les CIAL installent ce qu'ils appellent des banques de semences communautaires où ils conservent les variétés locales de leurs communautés mais aussi des communautés environnantes ainsi que les variétés améliorées qu'ils mettent progressivement au point (plus de 70 variétés locales de haricot, une quarantaine de maïs, une vingtaine de variétés améliorées, des semences de légumineuses, d'arbres, etc). Des échantillons des variétés conservées dans les communautés de ces montagnes sont entreposés également dans la banque de germoplasme national hébergée dans le centre de recherche qui les appuie. Ce même centre est lui-même connecté avec le niveau international, la banque de germoplasme (CIAT pour le cas du haricot). Cette interconnexion facilite la gestion-préservation de l'agrobiodiversité in situ et ex situ.

Il est éclairant de replacer leurs travaux dans le temps. À partir de 2005, les CIAL avaient commencé à identifier, répertorier, inventorier la gamme de variétés que les paysans de la région semaient et à les stocker dans leurs banques. De là, ils ont identifié celles qui pouvaient leur servir de parents dans les croisements effectués avec des variétés provenant de la banque nationale. Ils ont ensuite multiplié les semences des variétés créées et approvisionné les paysans de leurs communautés. Les CIAL ont alors monté des foires de la biodiversité au cœur des agglomérations principales de leurs zones, rendant ainsi la réalité de l'agrobiodiversité plus visible pour un plus grand nombre de personnes, rurales et citadines, précisant son sens, son utilité et la façon de la gérer. Progressivement, en complément du travail accompli par les banques communautaires de semences, et en articulation avec elles, certains paysans se sont lancés dans la conservation individuelle de variétés. Ils ont été baptisés " gardiens de semence ». Pour ces communautés, c'est bien l'ensemble et l'enchainement de ces diverses opérations qui contribuent à la gestion (et enrichissement) de l'agrobiodiversité (Hocdé et al., 2010). 
sont capables d'innovation en agriculture - se trouvent aux fondements des législations sur les semences qui ont été adoptées dans le sillage de la révolution verte. Il s'ensuit que ces législations tendent à être biaisées en faveur des systèmes semenciers considérés « officiels » et ne prennent pas en considération le rôle joué par les systèmes semenciers « locaux » (considérés « officieux» ou « informels ») qui sont gérés par les agriculteurs eux-mêmes et qui font intervenir la production, la multiplication, la distribution, des échanges, des améliorations et la conservation des semences au niveau local.

Les législations concernant les semences doivent tenir compte de la diversité des besoins des systèmes agricoles. Elles doivent reconnaître la valeur des divers systèmes semenciers pour différentes situations, et reconnaître le rôle important des agriculteurs pour la conservation et l'utilisation durable des ressources génétiques végétales à des fins alimentaires et agricoles.

Les réglementations en matière de semences doivent être ré-examinées et amendées pour prendre en compte la nécessité d'encourager la conservation de l'agrobiodiversité in situ et on farm (sur l'exploitation). Elles doivent soutenir le développement et l'entretien de la diversité des systèmes agricoles (et, pour ce faire, elles doivent envisager la nécessité de faire coexister des systèmes semenciers diversifiés), élargir la palette de diversité génétique à la disposition des agriculteurs et promouvoir une utilisation plus étendue des variétés et des espèces locales, adaptées aux conditions locales.

\section{L'agrobiodiversité et les droits des agriculteurs}

La mise en œuvre des droits des agriculteurs est fondamentale pour permettre la conservation et l'utilisation durable de l'agrobiodiversité. Les droits des agriculteurs comprennent les droits collectifs au foncier et à la réforme agraire, à l'eau, aux ressources naturelles, à l'énergie, aux technologies appropriées, ainsi qu'à l'éducation, aux soins médicaux, à la participation politique et à la liberté d'association, entre autres choses. Ces droits sont intrinsèquement liés entre eux, mais ici nous choisirons de nous concentrer sur les droits des agriculteurs ainsi qu'ils ont été définis dans le préambule et dans l'article 9 du Traité international sur les ressources phytogénétiques pour l'alimentation et l'agriculture (International Treaty on Plant Genetic Resources for Food and Agriculture ou ITPGRFA). Cet instrument constitue une importante opportunité pour élaborer et mettre en œuvre les droits des agriculteurs à l'échelon national, dans la mesure où il s'agit du premier traité international juridiquement contraignant qui reconnaisse explicitement les droits des agriculteurs. Nous détaillerons ensuite les principales dispositions relatives aux droits des agriculteurs, ainsi qu'elles sont présentées dans l'article 9 de ce traité.

«Article 9.1. Les parties contractantes reconnaissent l'énorme contribution que les communautés locales et autochtones ainsi que les agriculteurs de toutes les régions du monde, et spécialement ceux des centres d'origine et de diversité des plantes cultivées, ont apportée et continueront d'apporter à la conservation et à la mise en valeur des ressources phytogénétiques qui constituent la base de la production alimentaire et agricole dans le monde entier.

Article 9.2. Les parties contractantes conviennent que la responsabilité de la réalisation des droits des agriculteurs, pour ce qui est des ressources phytogénétiques pour l'alimentation 
et l'agriculture, est du ressort des gouvernements. En fonction de ses besoins et priorités, chaque partie contractante devrait, selon qu'il convient et sous réserve de la législation nationale, prendre des mesures pour protéger et promouvoir les droits des agriculteurs, y compris :

- la protection des connaissances traditionnelles présentant un intérêt pour les ressources phytogénétiques pour l'alimentation et l'agriculture ;

- le droit de participer équitablement au partage des avantages découlant de l'utilisation des ressources phytogénétiques pour l'alimentation et l'agriculture ;

- le droit de participer à la prise de décisions, au niveau national, sur les questions relatives à la conservation et à l'utilisation durable des ressources phytogénétiques pour l'alimentation et l'agriculture.

Article 9.3. Rien dans cet article ne devra être interprété comme limitant les droits que peuvent avoir les agriculteurs de conserver, d'utiliser, d'échanger et de vendre des semences de ferme ou du matériel de multiplication, sous réserve des dispositions de la législation nationale et selon qu'il convient. »

Bien que ce traité reconnaisse que les pays doivent prendre des mesures pour protéger les droits des agriculteurs, chaque pays peut décider des mesures à mettre en place, et les politiques et les actions qui figurent dans le traité n'y sont qu'à titre indicatif, laissant aux États la possibilité d'opter pour d'autres. Le préambule au traité mentionne explicitement le droit des agriculteurs à « conserver, utiliser, échanger et vendre des semences de ferme et d'autres matériels de multiplication ». Cependant, il est affirmé plus loin, dans l'article 9.3, que « rien dans cet article ne devra être interprété comme limitant les droits que peuvent avoir les agriculteurs de conserver, d'utiliser, d'échanger et de vendre des semences de ferme ou du matériel de multiplication, sous réserve des dispositions de la législation nationale et selon qu'il convient ». Ainsi, bien que les droits des agriculteurs soient affirmativement reconnus dans le préambule, l'article 9.3 reste neutre et précise seulement que la décision est à prendre par chaque pays, en fonction de sa législation nationale. L'article 9.3 reflète le manque de consensus qui existait entre les pays favorables à une reconnaissance effective des droits des agriculteurs à conserver, utiliser, échanger et vendre des semences produites sur leurs exploitations, et les pays qui étaient opposés à une telle reconnaissance, susceptible d'entraîner une restriction des droits des obtenteurs professionnels.

L'article 9.3 n'impose toutefois aucune restriction quant aux options qui peuvent être adoptées par les pays en matière d'application des droits des agriculteurs au niveau national, même si cela comprend une limitation des droits de la propriété intellectuelle sur les variétés végétales. Il s'agit sans doute là d'une des questions les plus controversées en ce qui concerne la mise en œuvre des droits des agriculteurs. Ni la proposition de l'Union européenne ni celle des États-Unis soumises à l'occasion de l'élaboration du Traité international sur les ressources phytogénétiques pour l'alimentation et l'agriculture ne faisaient allusion aux droits des agriculteurs à conserver, utiliser, échanger et vendre les semences produites sur les exploitations.

Or, pour la conservation et l'utilisation durable de la biodiversité, il est fondamental de veiller à ce que suffisamment d'espace juridique subsiste dans les législations sur les semences et dans les droits de la propriété intellectuelle pour que les agriculteurs puissent continuer à conserver, utiliser, échanger et vendre les semences et le matériel de propagation qui sont produits sur leurs exploitations. Il est en outre 
important de noter que ces semences de ferme comprennent tant des variétés traditionnelles que des variétés améliorées qui ont fait l'objet d'un travail de sélection et d'amélioration supplémentaire par les agriculteurs.

Pour veiller à ce que les plantes cultivées bénéficient d'une base génétique diversifiée, les législations sur les semences et les droits de la propriété intellectuelle doivent, les unes comme les autres, contenir des exceptions qui permettent aux agriculteurs petits producteurs ou producteurs locaux de conserver, d'utiliser, d'échanger et de vendre des semences et autres matériels de multiplication produits sur place à d'autres agriculteurs petits producteurs ou producteurs locaux, du moment que ces activités se déroulent sur les marchés locaux et entre agriculteurs locaux.

\section{Encadré 8.2. Le rôle des curadoras mapuches dans la conservation des variétés de quinoa}

\section{Max Thomet et Didier Bazile}

Le quinoa (Chenopodium quinoa Willd.) a été domestiqué il y a environ 7000 ans dans les Andes, région dans laquelle il est resté un aliment de base des populations indigènes de Colombie, Équateur, Pérou, Bolivie et Chili. Cette culture très rustique a su s'adapter à une grande diversité de milieux. Bien que cette plante soit souvent associée dans les esprits à l'altiplano où elle est majoritairement cultivée par les indiens aymaras, le quinoa fait aussi partie de la culture ancestrale des indiens mapuches du sud du Chili. Des traces archéologiques montrent une domestication récente entre 1200-1 400 après J.C. Base de leur alimentation par le passé, beaucoup moins consommé aujourd'hui, il reste toujours utilisé pour fabriquer le Mudä̈, une boisson consommée lors des cérémonies religieuses. Variétés des Incas ou variétés endémiques, le quinoa mapuche, aussi appelé Kinwa ou Dawe, n'a donc jamais complètement disparu malgré l'introduction de nombreuses espèces exotiques lors de la conquête espagnole.

Le Tukun est le concept mapuche pour nommer le potager (la huerta) où les indigènes cultivaient auparavant de très petites surfaces. Il reflète la cosmogonie du peuple mapuche dans sa forme, sa disposition et les types de plantes qui le composent, l'application du calendrier lunaire pour les semis et le repiquage, et la transmission orale des connaissances. Le potager mapuche était auparavant inséré dans les éléments forestiers comme autant de microcosmes alimentaires en parfait mimétisme dans le paysage. Jusqu'à nos jours, la responsabilité du potager incombe encore aux femmes, prendre soin des enfants ou des plantes correspondant à une qualité jugée féminine. C'est ainsi que durant des milliers d'années, des semences ont été collectées, échangées et adaptées dans des espaces semi-contrôlés. L'autoproduction et l'échange de semences sont un des mécanismes qui permettent de maintenir, voire d'augmenter, la diversité d'une année à l'autre. Comme cela a pu être montré aussi ailleurs dans le monde dans différents systèmes traditionnels très complexes d'échanges, au niveau des communautés mapuche, il a été mis en évidence que certains paysans participaient avec une fréquence plus élevée que les autres aux échanges. Ils comptent aussi une plus grande diversité d'espèces et de variétés dans leurs parcelles que le reste de la communauté. Ces personnes ressources pour l'agrobiodiversité assument pour le groupe le rôle de curadoras. Elles sont à la fois les gardiennes des semences et doivent protéger les plantes, mais elles sont aussi les détentrices des savoirs alimentaires, médicinaux ou autres pour lesquels elles ont la 
responsabilité d'assurer une continuité dans la chaîne de transmission des savoirs et des ressources génétiques pour que les variétés perdurent dans le temps.

Dans les communautés mapuche du sud Chili, le quinoa reste cultivé à $85 \%$ par les femmes qui sèment chacune en moyenne entre 2 et 4 variétés paysannes. Il existe aujourd'hui deux types d'échanges qui représentent l'accès principal aux semences. Le premier est un échange « individuel » (entre personnes ou familles) à l'intérieur de la communauté, basé sur une confiance étroite pour une transmission dans le secret. Le second est une sorte de marché traditionnel (Trafkintü) organisé sous forme d'événements publics (dans le sens d'une ouverture à différentes communautés) avec une cérémonie d'introduction et la présentation de chaque participant. Les curadoras correspondent aux nouds des réseaux d'échanges de semences c'est pourquoi elles contrôlent les deux types d'échanges.

Aujourd'hui, ces femmes curadoras participent activement comme formatrice dans les actions de conservation de la biodiversité. Elles travaillent en particulier en lien avec l'ANAMURI (Asociación Nacional de Mujeres Rurales e Indígenas) à la défense des semences paysannes dans le cadre de la campagne nationale Semillas campesinas : Patrimonio de los pueblos al servicio de la Humanidad. Des événements ont lieu chaque jour dans tous les territoires du pays ; ils reposent sur les stratégies propres des communautés paysannes comme les foires et échanges de semences (Trafkintü en Mapudungun, la langue mapuche), ou encore la participation à des séminaires ou à des journées de mobilisations publiques. Ces dernières années, le point focal de leurs actions a concentré tous les efforts sur le rejet de l'adoption des conventions et traités internationaux tels que le passage à l'acte de 1991 de l'UPOV (Union pour la protection des obtentions végétales) et les lois nationales chiliennes qui y sont liées car elles restreignent le droit ancestral au libre accès et à l'échange des semences.

En conclusion, la prise en compte et la valorisation de ces connaissances locales de gestion et de maintien de l'agrobiodiversité par les communautés agricoles traditionnelles est fondamentale. Le développement de nouvelles stratégies de conservation in situ de la biodiversité se doit d'intégrer ces savoirs et pratiques afin d'inverser les processus actuels d'érosion génétique générés par l'agriculture conventionnelle et les systèmes semenciers, privés ou étatiques, dits modernes (Aleman et al., 2010 ; Martínez et al., 2010).

La définition de ce qu'est un marché « local » est complexe et doit prendre en considération les dimensions socioculturelles autant que politico-administratives et agronomiques. Plusieurs intervenants ont déjà soumis des propositions visant à mieux équilibrer les droits de la propriété intellectuelle et les droits des agriculteurs à conserver, utiliser, échanger et vendre des semences de ferme (de variétés protégées), par exemple en limitant les droits des agriculteurs aux plantes cultivées destinées à la consommation nationale (ce qui exclut les productions destinées à l'exportation) ou, alternativement, à l'alimentation humaine et animale (ce qui exclut notamment les plantes ornementales). Ces deux propositions sont l'une et l'autre juridiquement et politiquement applicables et devraient être étudiées sérieusement par les pays parties au Traité international de la FAO sur les ressources phytogénétiques pour l'alimentation et l'agriculture dans le cadre de l'application des droits des agriculteurs au niveau national. 


\section{L'agrobiodiversité et la législation sur le patrimoine culturel}

Pour Sauer (1986), les plantes cultivées sont des « objets façonnés culturels », tandis que Emperaire et al. (2008) les considèrent comme des « objets de nature biologique, mais d'essence culturelle ». La culture est d'ailleurs présente à l'intérieur du terme «agriculture », et un des sens du mot «culture » est bien la culture de la terre. La culture et l'agriculture sont de ce fait intimement liées, et il est possible d'utiliser des instruments juridiques de protection du patrimoine culturel pour reconnaître et soutenir les systèmes agricoles à agrobiodiversité élevée et toutes leurs composantes, aussi bien matérielles (les agroécosystèmes et les plantes cultivées, par exemple) qu'immatérielles (les techniques, les pratiques, les savoirs agricoles, etc.).

La sauvegarde des habitudes alimentaires traditionnelles et de la diversité des régimes alimentaires constitue un autre moyen de soutenir l'agrobiodiversité et la sécurité alimentaire. La préservation de la diversité génétique des plantes cultivées ne peut être dissociée de l'utilisation de ces plantes, et promouvoir les régimes alimentaires traditionnels, qui sont souvent plus diversifiés, contribue à sauvegarder ensemble le patrimoine biologique et culturel de l'humanité. Il en découle que les instruments juridiques de préservation du patrimoine culturel sont susceptibles d'être utilisés (et l'ont d'ailleurs été dans certains cas) pour œuvrer en faveur de l'agrobiodiversité et de la diversité alimentaire par des moyens innovants : c'est notamment le cas de la convention concernant la protection du patrimoine mondial, culturel et naturel et de la convention pour la sauvegarde du patrimoine culturel immatériel de l'Unesco (Organisation des Nations unies pour l'éducation, la science et la culture).

Depuis ses origines, la liste représentative du patrimoine culturel immatériel de l'humanité de l'Unesco a été essentiellement orientée vers les arts de la scène et les artisanats. Plus récemment, l'Unesco semble avoir accepté l'idée que les traditions culinaires sont une expression culturelle aussi fondamentale, pour l'identité culturelle, et aussi digne de reconnaissance que la danse, le théâtre et la musique. Comme le patrimoine alimentaire est directement lié à la diversité génétique des plantes cultivées, il est intéressant de rappeler que trois systèmes culinaires ont été ajoutés à la liste en 2010 : la cuisine traditionnelle mexicaine, les régimes alimentaires méditerranéens d'Espagne, de Grèce, d'Italie et du Maroc, et le repas gastronomique des français. L'Unesco œuvre également en partenariat avec Bioversity International et avec le ministère kényan du Patrimoine national et de la culture sur un projet appelé Safeguarding Traditional Foodways of Two Communities in Kenya (sauvegarde des habitudes alimentaires traditionnelles de deux communautés au Kenya). Dans le cadre de ce projet, il est reconnu que les habitudes alimentaires, tant quotidiennes qu'associées à des événements particuliers (tels que des rituels ou des festivités), sont une composante importante du patrimoine immatériel des populations locales.

Une autre initiative allant dans le même sens est née au Brésil. Le 8 novembre 2010, l'Institut national du patrimoine artistique et historique (IPHAN, l'agence fédérale brésilienne pour la protection du patrimoine culturel) a reconnu pour la première fois un système agricole traditionnel à agrobiodiversité élevée, celui de la région du Rio Negro (nord-ouest de l'Amazonie brésilienne), comme « patrimoine culturel immatériel du Brésil ». Selon Emperaire et al. (2008), dans le contexte du Rio Negro, 
Encadré 8.3. De la ressource phytogénétique au patrimoine culturel : les bases sociales de la gestion de l'agrobiodiversité en Amazonie brésilienne

\section{Ludivine Eloy et Laure Emperaire}

La région amazonienne peut être qualifiée de mégadiverse par sa diversité biologique spontanée et par celle en plantes cultivées, sélectionnées et maintenues par les populations locales. La pérennité de cette agrobiodiversité est aujourd'hui remise en question par la diffusion de modèles et de techniques agricoles qualifiés de modernes, le développement des infrastructures, l'urbanisation etc. Les systèmes semenciers traditionnels sont en voie d'appauvrissement tant sur le plan des ressources phytogénétiques en jeu que sur celui des connaissances et pratiques qui y sont associées.

Le programme « Populations locales, agrobiodiversité et connaissances traditionnelles associées » (Pacta), mené en coopération entre l'Unicamp et l'IRD, coordonné par Mauro Almeida et Laure Emperaire, s'interroge sur le devenir de ces agricultures à faibles intrants et sur l'agrobiodiversité qui y est associée.

La recherche menée en Amazonie brésilienne, en particulier dans la région du Rio Negro, vise à comprendre les bases sociales et culturelles de tels systèmes. L'originalité de la démarche proposée n'est pas de s'intéresser aux savoirs en tant que corpus de données opérationnelles mais de tenter de comprendre leur signification sociale et les valeurs dont ils sont porteurs. Nous mettons en évidence que l'amplitude de la diversité gérée par les populations amérindiennes, dépasse largement des exigences fonctionnelles, agronomiques ou alimentaires. L'analyse des réseaux de circulation de l'ensemble des plantes cultivées des unités familiales met en évidence deux niveaux de gestion de la diversité agricole. Le premier opère au sein de l'exploitation : il est imposé par le fonctionnement de l'agriculture sur brûlis qui requiert le transfert annuel du germoplasme de l'ancien abattis à celui nouvellement défriché. Le maillage du deuxième réseau se situe à l'échelle régionale, parfois sur des centaines de kilomètres. L'enjeu de l'échange dépasse la seule obtention d'une variété nouvelle, la plante est aussi le vecteur de significations sociales. Avoir de nombreuses espèces ou variétés cultivées est synonyme de prestige et d'une sociabilité accomplie. La diversité des statuts des plantes cultivées au sein de la société est révélée par la forme de cette circulation : don, échange ou transmission. Ces modèles conceptuels locaux se heurtent aux savoirs formalisés au sein des institutions de développement agricole, ou les complètent. La question est d'identifier comment ces stratégies locales de gestion de la biodiversité peuvent être reconnues et valorisées en termes d'apport à la conservation des ressources phytogénétiques (Emperaire et al., 2010 ; Cardoso et al., 2010).

un système agricole peut être compris comme « un ensemble de savoirs, de mythes, d'expressions orales, de pratiques, de produits, de techniques, d'objets manufacturés et d'autres manifestations et expressions associées qui font intervenir une gestion des espaces, la culture de végétaux, la transformation des produits agricoles, et les systèmes alimentaires locaux. " Ces auteurs expliquent que la notion de système permet de relier le patrimoine culturel (au nom de quoi une reconnaissance est recherchée) à un ensemble plus complexe et plus large de relations sociales. Pour eux, l'enregistrement du système agricole du Rio Negro comme patrimoine culturel immatériel est « un exemple concret qui montre comment des instruments et des 
politiques de protection du patrimoine culturel peuvent être utilisés en faveur de l'agrobiodiversité, de la diversité culturelle et des systèmes agricoles locaux. »

Le 23 décembre 2009, le Pérou a émis une Résolution directoriale nationale (Resolución Directoral Nacional N N $^{\circ}$ 1986/INC) déclarant «patrimoine culturel national » les savoirs, les pratiques et les techniques associés à la culture traditionnelle du maïs dans la vallée sacrée des Incas, dans les Andes péruviennes. C'était la première fois au Pérou qu'un système agricole était reconnu patrimoine culturel. Selon cette Résolution, le maïs, ou sara en langue Quechua, bien que d'origine méso-américaine, a été cultivé dans les Andes péruvienne depuis des millénaires, et cette céréale est représentée dans la région par 55 variétés.

Il est également intéressant de relever qu'en 1992, la convention de l'Unesco concernant la protection du patrimoine mondial, culturel et naturel a mis en place une nouvelle catégorie, innovante, de sites protégés, celle de « paysage culturel ». Ces « paysages culturels » sont habilités à figurer dans la liste du patrimoine mondial de l'Unesco lorsque les interactions entre la population et leur environnement sont considérés comme ayant « une valeur universelle exceptionnelle ». Plusieurs paysages agricoles importants ont d'ores et déjà été inscrits sur cette liste en tant que "paysages culturels ", notamment les rizières en terrasses des cordillères des Philippines, le paysage archéologique des premières plantations de café du sud-est de Cuba, le paysage agricole du sud d'Öland en Suède, et le paysage pastoral de la puszta du Parc national de Hortobágy en Hongrie. Ces exemples illustrent la possibilité d'utiliser la catégorie des " paysages culturels » pour promouvoir et sauvegarder les sites d'agrobiodiversité patrimoniale (Singh et Varaprasad, 2008). Ainsi est-il possible de préserver le riche patrimoine génétique des plantes cultivées et des animaux d'élevage associés aux agroécosystèmes à diversité élevée, sans oublier les techniques, les pratiques et les innovations agricoles des populations locales, non seulement par le biais d'instruments relevant de la législation sur l'environnement, mais encore en faisant appel aux outils de sauvegarde du patrimoine culturel tels que ceux mentionnés plus haut.

\section{L'agrobiodiversité et les aires protégées}

Le rôle des aires protégées dans la conservation et l'utilisation durable de l'agrobiodiversité a été considérablement sous-estimé. Au niveau international, il n'existe toujours pas de catégorie d'aire protégée qui soit spécifiquement consacrée à la sauvegarde de l'agrobiodiversité, qu'il s'agisse de la conservation in situ d'espèces sauvages parentes d'espèces cultivées, ou de la gestion, en exploitation (on farm) de systèmes à agrobiodiversité élevée (bien que d'autres catégories de sites protégés aient pu occasionnellement servir à la conservation de l'agrobiodiversité).

Les aires protégées ont vu leur effectif mondial passer d'environ 56000 en 1996 à près de 70000 en 2007, tandis que leur superficie totale passait de 13 millions à 17,5 millions de $\mathrm{km}^{2}$ au cours de la même période - mais il reste que, selon le $2^{\mathrm{e}}$ Rapport sur l'état des ressources phytogénétiques pour l'alimentation et l'agriculture dans le monde (FAO, 2010, p. 34), les secteurs accueillant l'agrobiodiversité la plus riche (notamment les centres d'origine et/ou de diversité) sont significativement moins protégés que dans la moyenne mondiale. Ce même rapport précise 
qu'un nombre significatif de ressources phytogénétiques pour l'alimentation et l'agriculture, dont des espèces sauvages parentes d'espèces cultivées et des plantes utiles récoltées dans la nature, se trouvent à l'extérieur de zones protégées (dans des champs cultivés, des vergers, des prairies, etc.) et ne bénéficient de ce fait d'aucune forme de protection juridique.

Comme l'ont déjà fait remarqué plusieurs auteurs (Phillips, 2005 ; Rössler, 2005 ; Santilli, 2005), un glissement s'est opéré dans le paradigme qui sous-tend les efforts de conservation, passant de la désignation de sites naturels exceptionnels sans occupation humaine (zones primaires ou quasi primaires) à la reconnaissance de la valeur de sites de patrimoine naturel dans un contexte paysager comportant une population humaine résidente. Autrement dit, plus d'attention est accordée à l'interaction entre les humains et la nature, et l'importance de sites protégés centrés sur des paysages habités est de plus en plus admise et acceptée. Dans le sillage de cette évolution, une nouvelle catégorie d'aire protégée, de type « réserve d'agrobiodiversité » ou « paysage protégé à agrobiodiversité élevée » pourrait être instaurée, spécifiquement axée sur la gestion de la biodiversité agricole et sur la reconnaissance des techniques, des pratiques et des savoirs agricoles traditionnels. La plupart des initiatives visant à préserver l'agrobiodiversité au sein d'aires protégées sont mises en œuvre dans des parcs ou des réserves biologiques qui n'étaient pas conçus au départ pour la sauvegarde des cultures et des zones cultivées. Il existe toutefois quelques exceptions, dont notamment le Parque de la Papa, créé par des communautés indigènes du Pérou, et la réserve de la Sierra del Manantlán, au Mexique.

La création d'une catégorie d'aire protégée reconnue à l'échelon international et spécialement destinée à préserver l'agrobiodiversité contribuerait à une meilleure prise de conscience générale de l'importance de la diversité agricole et de ses implications pour la sécurité alimentaire, l'alimentation, la santé, l'équité sociale et la durabilité environnementale. Pendant longtemps, la plupart des politiques et des démarches de conservation ont été concentrées sur la biodiversité sauvage, comme s'il était plus important pour l'humanité de sauvegarder les espèces végétales et animales sauvages que la diversité des plantes cultivées telles que le riz, les haricots, le blé ou la pomme de terre, qui figurent quotidiennement dans notre alimentation. Qui plus est, le manque d'intégration des approches utilisées par les politiques agricoles et environnementales est bien souvent à l'origine d'un cloisonnement et d'une incoordination des efforts de préservation des espèces et des écosystèmes sauvages, d'une part, et de conservation ex situ d'espèces cultivées ou domestiquées, d'autre part, respectivement consentis par des agences de protection de l'environnement et par des institutions de recherche agronomique n'interagissant pratiquement pas entre elles.

La création de réserves d'agrobiodiversité permettrait en outre d'attirer un peu plus l'attention des décideurs sur la pertinence d'identifier les hotspots de l'agrobiodiversité, de prospecter et d'inventorier les zones à haute diversité agricole (notamment en ce qui concerne les espèces sauvages parentes d'espèces cultivées, les variétés et races locales ou traditionnelles, et les systèmes agricoles ingénieux), et de définir les critères et les méthodes à employer pour l'identification et la gestion adaptative de ces sites. Des initiatives de ce type contribueraient à améliorer la compréhension scientifique de la gestion in situ, sur l'exploitation (on farm), de l'agrobiodiversité, 
et à mieux faire reconnaître la valeur des systèmes semenciers locaux et des réseaux sociaux pour le maintien de la diversité génétique végétale. Une catégorie d'aire protégée spécialement conçue pour encourager la gestion de l'agrobiodiversité sur les exploitations mêmes (on farm) devrait permettre une utilisation durable des ressources phytogénétiques et orienter les efforts sur la gestion adaptative et sur les interactions entre la population humaine et le milieu naturel.

Étant donné que ce sont les systèmes agricoles traditionnels et locaux qui préservent et gèrent l'essentiel de l'agrobiodiversité, il serait possible de créer une réserve d'agrobiodiversité uniquement avec le soutien et l'engagement des agriculteurs résidant et travaillant dans la zone sélectionnée. La participation des agriculteurs serait indispensable pour la mise au point et l'application des plans de gestion et des actions, qui devraient promouvoir une approche intégrée (et complémentaire) des systèmes de connaissances scientifiques et traditionnels. Au lieu de se limiter à la simple conservation de l'environnement, les réserves d'agrobiodiversité devraient, afin de se donner une durabilité sociale et politique, notamment dans les pays en voie de développement, satisfaire des objectifs plus ambitieux de développement durable local et d'inclusion sociale.

Elles peuvent être établies sur des terres qui ne sont pas propriété publique. Toutefois, il serait évidemment incohérent d'exproprier les propriétaires exploitants éventuellement localisés à l'intérieur de leur périmètre. Elles pourraient être mises en place par des accords avec les agriculteurs locaux qui résident dans le secteur désigné et qui exploitent ces agroécosystèmes à agrobiodiversité élevée. Il serait envisageable d'octroyer une compensation aux agriculteurs concernés pour leurs contributions à la conservation de la biodiversité agricole, par exemple sous la forme d'un paiement pour services environnementaux rendus.

\section{L'agrobiodiversité et les systèmes ingénieux du patrimoine agricole mondial (Sipam)}

En 2002, la FAO a lancé une initiative pour la préservation et la gestion adaptative des systèmes ingénieux du patrimoine agricole mondial (Sipam). Ce projet a pour objectif de jeter les fondations de la reconnaissance internationale, de la conservation dynamique et de la gestion adaptative de ces Sipam et de la biodiversité agricole, des systèmes de connaissances, de la sécurité alimentaire, de la sécurité des moyens de subsistance, et des cultures qui leur sont associés, partout sur la planète. Ces systèmes ingénieux sont définis comme étant « des systèmes et des paysages remarquables d'exploitation de terres riches en une diversité biologique d'importance mondiale et évoluant grâce à l'adaptation d'une communauté à son environnement, à ses besoins et à ses aspirations au développement durable ». Le programme Sipam est financé par le Fonds pour l'environnement mondial (FEM) par l'intermédiaire du programme des Nations unies pour le développement (PNUD). Parmi ses autres partenaires figurent l'Unesco, le Centre international d'études pour la conservation et la restauration des biens culturels (ICCROM), l'Université des Nations unies (UNU), le Fonds international de développement agricole (FIDA), Bioversity International et l'Union internationale pour la conservation de la nature (UICN). 
Des universités, le secteur privé et des organisations de la société civile participent également à des projets pilotes.

Le concept de systèmes ingénieux est à distinguer de celui de site patrimonial ou de paysage protégé (protected landscape) conventionnel, et il est également plus complexe. Bien que les promoteurs du projet Sipam reconnaissent que l'expérience de l'Unesco dans l'identification des sites et des paysages du patrimoine mondial, notamment en ce qui concerne la catégorie des paysages vivants essentiellement évolutifs, se soit avérée utile pour favoriser l'agrobiodiversité, ils considèrent que cette catégorie aurait besoin d'intégrer une vision plus sensible aux aspects agricoles et une approche axée sur les systèmes d'utilisation des terres. Le projet Sipam, qui œuvre à instaurer un mécanisme de reconnaissance de ces systèmes ingénieux qui soit accepté à l'échelon international, examine actuellement la possibilité d'inclure des systèmes agricoles dans les catégories existantes de paysages culturels ou de créer une nouvelle catégorie de patrimoine mondial. Ce projet vise à faciliter l'intégration de la conservation de l'agrobiodiversité au sein des politiques et des plans nationaux relatifs à la biodiversité, dans le but de renforcer les capacités des pays (notamment de ceux accueillant des systèmes pilotes) à promouvoir une utilisation durable de l'agrobiodiversité. Les populations pratiquant une agriculture paysanne familiale traditionnelle et les peuples indigènes en seraient les principaux bénéficiaires. L'objectif est de créer un réseau de systèmes ingénieux et de lancer un programme à long terme et évolutif. Environ 200 systèmes agricoles ont d'ores et déjà été identifiés, et des systèmes pilotes ont été établis dans un certain nombre de pays :

- 1. le système agricole des Andes, des vallées de Cuzco et de Puno, non loin de la cité Inca de Machu Picchu (Pérou). Cette région, où les cultures sont réalisées en terrasses, parfois jusqu'à $4000 \mathrm{~m}$ d'altitude, est le centre d'origine de la pomme de terre (domestiquée par les peuples indigènes Aymara et Quechua), du quinoa, du quinquina, de la coca, de l'amaranthe, du piment et de légumes-racines, tels que la arracacha et le yacón, qui tiennent une place fondamentale dans les régimes alimentaires de la région ;

- 2. le système agricole de l'archipel de Chiloé, dans le sud du Chili, est un centre d'origine et de diversité de la pomme de terre. Environ 200 variétés locales de cette espèce y sont encore cultivées par le peuple indigène Huilliche, ainsi qu'une variété d'ail appelé ajo chilote qui n'existe que sur les terres volcaniques de cet archipel ;

-3. les rizières en terrasses de la province d'Ifugao, dans les montagnes philippines, qui figurent également sur la liste des sites du patrimoine mondial de l'Unesco, dans la catégorie des « paysages culturels » de la Convention du patrimoine mondial. Il s'agit d'un agroécosystème situé en zone montagneuse d'altitude, au sein duquel les terrasses épousent les courbes du relief en formant un ensemble de micro bassins hydrographiques qui fonctionnent comme des systèmes d'irrigation et de filtration. Il est estimé que 565 variétés de riz y sont préservées (Nozawa et al., 2008) ;

- 4. les systèmes agricoles des oasis de la région du Maghreb (Algérie, Maroc et Tunisie). Les oasis du Maghreb constituent des îlots de végétation verdoyante dans un milieu naturel caractérisé par de très fortes contraintes. Elles abritent un système de production agricole intensif, très diversifié et hautement productif qui 
a été élaboré sur plusieurs millénaires. Elles produisent des dattes, d'autres fruits (grenades, figues, pêches, pommes, raisin, etc.), des légumineuses, des céréales, des légumes, des plantes médicinales, etc. L'oasis de Tamegroute, au Maroc, figure par ailleurs au programme sur « l'Homme et la biosphère » de l'Unesco et fait partie de la réserve de biosphère des oasis du sud marocain ;

-5 . le système de pisciculture-riziculture en Chine. Les poissons sont élevés en symbiose avec les rizières inondées. Ils apportent en effet la fumure pour la croissance du riz, régulent les conditions microclimatiques du milieu, ameublissent le substrat, agitent l'eau et consomment les larves et les mauvaises herbes, tandis que les plants de riz leur fournissent de l'ombre et de la nourriture. Ce système traditionnel existe depuis la dynastie des Han, il y a 2000 ans ;

-6 . les rizières en terrasses des Hani (Chine). Ces rizières se trouvent dans la préfecture autonome Hani et Yi de Honghe, dans le sud-est de la Province du Yunnan. Le paysage écologique se compose typiquement d'un assemblage de zones forestières, de zones d'habitations, et de zones de terrasses surplombant les rivières. La minorité ethnique Hani vit dans cet environnement depuis plus de 1300 ans ;

-7 . le système traditionnel de riziculture de Wannian (Chine). Le riz traditionnel de Wannian est une variété remarquable, très ancienne et hautement spécialisée, qui ne peut se développer que dans l'eau, le sol et les conditions climatiques particulières des environs du village de Heqiao. Cette variété a besoin d'être irriguée en permanence par de l'eau de source froide. Les forêts environnantes jouent un rôle crucial dans la préservation des sols et de la ressource en eau. L'ensemble des forêts et des rizières font partie du même système agroforestier à biodiversité élevée ;

-8 . le système pastoral des Masaï et l'agroécosystème des hauts plateaux (Kenya et Tanzanie). Plus de $75 \%$ de la population africaine réside en zone rurale, dans des petits villages et sur des exploitations familiales. Ce projet vise à renforcer la viabilité des petites exploitations, de l'agriculture traditionnelle et des systèmes agropastoraux, et à renforcer la sécurité alimentaire et nutritionnelle des populations indigènes qui dépendent de ces systèmes au Kenya et en Tanzanie. Le projet a pour objectif la gestion adaptative et la préservation des paysages productifs des populations Masaï et Tapade.

Le programme Sipam vise à identifier, définir et soutenir les formes de sauvegarde et de gestion dynamique de ces systèmes agricoles afin de permettre aux agriculteurs locaux d'entretenir la diversité biologique tout en préservant les ressources naturelles indispensables à leur survie. Il œuvre à mettre au point des politiques et des incitations publiques pour la conservation de la biodiversité, et des savoirs traditionnels associés, in situ et sur les exploitations (on farm). Un des traits caractéristiques de ces systèmes agricoles traditionnels est justement l'agrobiodiversité particulièrement élevée qu'ils entretiennent : au moins 177 variétés de pommes de terre ont été inventoriées dans les Andes péruviennes, environ 20 variétés traditionnelles de riz dans les systèmes de pisciculture-riziculture en Chine et plus de 10 variétés de dattes dans les oasis du Maghreb. Tout en faisant reconnaître la valeur de ces systèmes agricoles traditionnels ou locaux et en ouvrant à leur sauvegarde, le programme Sipam constitue par ailleurs une source d'informations pour étayer les discussions concernant la création d'aires protégées spécialement dédiées à la sauvegarde de l'agrobiodiversité. 


\title{
" Conclusion
}

La complexité extrême de l'univers agraire et agricole de la planète tient non seulement à la diversité considérable des paysages et des écosystèmes (dotés de caractéristiques environnementales propres), mais encore à la grande variété des modes de subsistance et des stratégies de survie et de production des agriculteurs. Bien qu'il soit courant de considérer le monde agricole comme scindé en deux pôles contrastés, avec l'agrobusiness industriel d'une part et la petite paysannerie de l'autre, la vérité est que notre diversité agricole culturelle et biologique n'est pas constituée d'un simple binôme, mais d'une multiplicité de systèmes d'exploitation très divers (indigènes, traditionnels, familiaux, paysans, biologiques, ou encore monoculturaux). Le cadre juridique de l'agrobiodiversité est encore en chantier, à l'échelle internationale comme dans la plupart des pays, ce qui donne à toutes les parties prenantes sociales une chance de réfléchir et de discuter pour définir comment les politiques et la législation pourraient contribuer plus efficacement à la gestion et à l'utilisation durable de l'agrobiodiversité in situ et sur les exploitations (on farm), ainsi qu'à la sécurité alimentaire, à la diversité culturelle et à l'équité sociale (Santilli, 2011). Bien des instruments juridiques dont il a été question dans ce chapitre ne sont pas encore finalisés ou sont en cours de révision. D'autres doivent être pensés et mis au point, ou, déjà en place à l'échelon international, restent à appliquer au niveau national. Pour les législateurs et les décideurs, un tel contexte est une belle occasion de faire preuve d'innovation en créant de nouveaux outils conçus pour renforcer les processus biologiques, sociaux et culturels complexes qui entretiennent et enrichissent l'agrobiodiversité.

\section{\# Références bibliographiques}

\begin{abstract}
Aleman J., Thomet M., Bazile D., Pham J.-L., 2010. Central role of nodal farmers in seed exchanges for biodiversity dynamics example of «Curadoras » for the quinoa conservation in mapuche communities in south Chile, In : Innovation and Sustainable Development in Agriculture and Food (ISDA), Montpellier, France - http://hal.archives-ouvertes.fr/hal-00530950/fr/
\end{abstract}

Bioversity International, 2010. Agriculture, agricultural biodiversity and sustainability, booklet based on the paper 'Sustainable Agriculture and the Sustainable Use of Agricultural Biodiversity: Concepts, Trends and Challenges', In : 14th meeting of the Convention on Biological Diversity's Subsidiary Body on Scientific, Technical and Technological Advice, Nairobi, Kenya.

Bonneuil C., Demeulenaere E., Thomas F., Joly P.B., Allaire G., Goldringer I., 2006. Innover autrement? La recherche face à l'avènement d'un nouveau régime de production et de régulation des savoirs en génétique végétale, In: Quelles variétés et semences pour des agricultures paysannes durables ? (Gasselin, P., Clèment O., eds), Les Dossiers de L'Environnement de l'Inra, no. 30, $29-52$.

Cardoso T., Eloy Pereira L., Emperaire L., 2010. Rôle des dynamiques spatio-temporelles dans la conservation de l'agrobiodiversité des systèmes agricoles amérindiens du bas rio Negro (Amazonas, Bresil), In : Innovation and Sustainable Development in Agriculture and Food (ISDA), Montpellier, France - http://hal.archives-ouvertes.fr/hal-00512235/fr

Cromwell E., Cooper D, Mulvany P., 2003. Defining agricultural biodiversity, In : Conservation and Sustainable Use of Agricultural Biodiversity: A Sourcebook, Centro Internacional de la Papa (CIP) and Users' Perspective with Agricultural Research and Development (UPWARD), Manila, vol. 1, chapter 1, pp. 1-12. 
Emperaire L., Velthem L.H.V., Oliveira A.G., 2008. Patrimônio cultural imaterial e sistema agrícola: o manejo da diversidade agrícola no médio Rio Negro (AM), In : 26th Meeting of the Brazilian Anthropology Association, June 1-4, 2008, Porto Seguro, Bahia, Brazil.

Emperaire L., Almeida M., Carneiro Da Cunha M., Eloy L., 2010. Innover, Transmettre. La diversité agricole en Amazonie brésilienne, In : Innovation and Sustainable Development in Agriculture and Food (ISDA), Montpellier, France - http://hal.archives-ouvertes.fr/hal-00512260

FAO, 1996. Rome Declaration on World Food Security and World Food Summit Plan of Action. World Food Summit, November 13-17, 1996, FAO, Rome.

FAO, 2010. Second Report on the State of the World's Plant Genetic Resources for Food and Agriculture, Commission on Genetic Resources for Food and Agriculture, FAO, Rome, http://www. fao.org/agriculture/crops/core-themes/theme/seeds-pgr/sow/en/ (consulté le 10 Decembre 2010).

GTZ (German Technical Cooperation), 2006. Agrobiodiversity - the key to food security', sector project 'People, Food and Biodiversity (Division 45), Germany.

Hocdé H., Rosas J.C. Araya R., 2010. Co-desarrollo de variedades entre agricultores, científicos y profesionales, biodiversidad y otras cosas, In : Innovation and Sustainable Development in Agriculture and Food (ISDA), Montpellier, France - http://hal.archives-ouvertes.fr/hal-00531488/ fr/

Louwaars N., 2007. Seeds of confusion: the impact of policies on seed systems, PhD dissertation, Wageningen, the Netherlands.

Louwaars N., 2010. Seed systems and plant genetic resources for food and agriculture: thematic background study, In : Second Report on the State of the World's Plant Genetic Resources, FAO, Rome.

Martínez E.A., Bazile D., Thomet M., Delatorre J., Salazar E., León Lobos P., Von Baer I., Núñez L., 2010. Neo-liberalism in chile and its impacts on agriculture and biodiversity conservation of quinoa: a lesson for strengthening and developing new partnerships, In : Innovation and Sustainable Development in Agriculture and Food (ISDA), Montpellier, France - http://hal.archives-ouvertes.fr/ hal-00523036/en/

Nozawa C., Malingan M., Plantilla A., Ong J., 2008. Evolving culture, evolving landscapes: the Philippine rice terraces, In: Protected Landscapes and Agrobiodiversity Values (Amend T., Brown J., Kothari A., Philipps A., Solton S., eds.), Values of Protected Landscapes and Seascapes, a series published by the Protected Landscapes Task Force of IUCN's World Commission on Protected Areas, IUCN, Gland, Switzerland.

Phillips A., 2005. Landscape as a meeting ground: Category V protected landscapes/seascapes and world heritage cultural landscapes, In : The Protected Landscape Approach: Linking Nature, Culture and Community (Brown J., Mitchell N., Beresford M., eds.), IUCN, Gland, Switzerland, Cambridge, UK, 19-35.

Rössler M., 2005. World heritage cultural landscapes: a global perspective, In : The Protected Landscape Approach: Linking Nature, Culture and Community (Brown J., Mitchell N., Beresford M., eds.), IUCN, Gland, Switzerland, and Cambridge, UK, 37-46.

Santilli J., 2005. Socioambientalismo e Novos Direitos: Proteção Jurídica à Diversidade Biológica e Cultural, Editora Peiropolis, ISA/IEB, São Paulo, Brazil.

Santilli J., 2011. Agrobiodiversity and the Law: Regulating Genetic resources, Food Security and Cultural Diversity, Earthscan, London.

Sauer C., 1986. As plantas cultivadas na América do Sul tropical, In : Suma Etnológica Brasileira: Etnobiologia (Ribeiro B., ed.), 3rd ed., Vozes, FINEP, Petrópolis, Brazil, 59-90.

Singh A., Varaprasad K.S., 2008. Criteria for identification and assessment of agrobiodivesity heritage sites: evolving sustainable agriculture. Current Science, 94 (9), 1131-1138 (http://cs-test.ias. ac.in/cs/Downloads/article_42630.pdf; accessed April 30, 2012). 



\title{
Chapitre 9
}

\section{Politiques pour promouvoir l'innovation, le cas de la Méditerranée}

\author{
Karim Hussein et Khalid El Harizi ${ }^{1}$
}

\section{Wes défis globaux et régionaux en matière d'agriculture et d'alimentation : l'impératif de l'innovation}

Il est communément admis aujourd'hui que l'innovation est cruciale dans le domaine des sciences, des technologies et des pratiques agricoles pour pouvoir relever les défis que rencontrent les systèmes alimentaires, en particulier dans les pays à revenu faible ou intermédiaire. Tous les échelons politiques reconnaissent le rôle déterminant des filières agroalimentaires performantes, mais également des petits exploitants familiaux, qui produisent la majeure partie des denrées dans les pays en voie de développement. Il devient de ce fait nécessaire de développer des partenariats innovants entre les divers acteurs tout au long des filières et des chaînes de valeur.

L'innovation en matière d'agriculture et d'alimentation, en particulier à destination des petits producteurs, se retrouve aujourd'hui en tête des préoccupations internationales et des priorités des politiques de développement. Â la suite des initiatives prises ces dernières années par le groupe des huit pays développés en matière de sécurité alimentaire ${ }^{2}$, les ministres du groupe des vingt pays industrialisés et en voie

1. Bien que l'analyse et les conclusions présentées dans ce chapitre demeurent sous l'entière responsabilité des auteurs, elles s'appuient sur des éléments dégagés à l'occasion des présentations et des discussions qui ont eu lieu pendant le symposium ISDA à Montpellier, dans le cadre de la table ronde « Politiques pour promouvoir l'innovation : le cas de la Méditerranée » organisée par l'Organisation de coopération et de développement économiques (OCDE), le Fonds international de développement agricole (FIDA) et ARIMNet, avec le soutien de la Fondation Agropolis. Les auteurs remercient chaleureusement pour leurs contributions les autres membres du panel : Prof. Mohamed Ait-Kadi (président du Conseil général du développement agricole, Maroc), Prof. Osama Momtaz (coordinateur pour le réseau biotechnologie agricole de l'Association of Agricultural Institutions in the Near East and North Africa/assistant directeur de recherche à l'Agriculture Genetic Engineering Research Institute-Agricultural Research Centre, Égypte), Claude Torre (Agence française de développement, France), et Gang Zhang (administrateur principal, direction de la science, de la technologie et de l'industrie, OCDE).

2. Voir par exemple les passages sur l'agriculture et la sécurité alimentaire du Rapport de l'examen mutuel de l'efficacité du développement en Afrique de la CEA/ONU et de l'OCDE qui détaillent les 
de développement à la Conférence du G20 de Montpellier (France) sur la recherche agricole pour le développement, en Septembre 2011, ont insisté sur l'importance de l'innovation, de la recherche et de la hausse de la productivité agricole, qui ont à nouveau été remises à l'honneur par les dirigeants du G20 au Sommet de Cannes (France) en novembre 2011. La nécessité d'encourager le partage des innovations avec les pays en voie de développement, et entre ces derniers, a été soulignée en ces occasions. Une priorité élevée a également été accordée à la résolution des problèmes de sécurité alimentaire et de volatilité des prix des denrées par le biais d'investissements dans les sciences et les technologies afin d'accroître la productivité et la mise en commun des connaissances.

Cette récente prise de conscience internationale résulte d'une combinaison de facteurs. En premier lieu, la crainte que la croissance démographique ne fasse progresser la demande alimentaire plus rapidement que les possibilités d'augmentation de la productivité agricole. En effet, une étude des Nations unies a indiqué que la population mondiale atteindrait 7 milliards en octobre 2011 et laisse entendre un dépassement des 9 milliards vers 2050. Selon une estimation du G20, la production agricole mondiale devra augmenter de $70 \%$ sur cette même période pour pouvoir nourrir l'humanité. Deuxièmement, les flambées et la volatilité récentes et marquées du prix des denrées ont suscité une inquiétude quant à la suffisance de l'approvisionnement alimentaire : les maximums des cours enregistrés en 2008 et 2010 ont entraîné des instabilités, des émeutes et des morts dans plusieurs pays à revenu faible ou intermédiaire (y compris en Afrique de l'Ouest et en Afrique du Nord). La tendance semble durable pour ce qui est des principales céréales et autres denrées de base sur les marchés internationaux et régionaux. Selon l'Organisation des Nations unies pour l'alimentation et l'agriculture (FAO) et l'OCDE, les prix demeureront environ de $20 \%$ supérieurs entre 2011 et 2020 à ce qu'ils étaient sur la période 2001-2010 (OCDE-FAO, 2011). Troisièmement, il est prévu que le changement et la variabilité climatiques auront des effets négatifs sur la production agricole et alimentaire, qui affecteront plus que les autres les régions arides et semi-arides telles que l'Afrique du Nord, le Sahel et la Méditerranée.

À ces préoccupations internationales viennent s'en ajouter d'autres : la concurrence exacerbée qui sévit au niveau des marchés globalisés et régionaux réclamant un accroissement de l'efficacité et de la productivité, la hausse des cours de l'énergie, qui se répercute sur le prix des intrants agricoles clés (tels que les engrais), et les situations caractérisées par des instabilités, des conflits, des institutions faibles, des inégalités et des problèmes de gouvernance. Ensemble, ce sont là des défis majeurs pour le développement durable dans l'agriculture et l'alimentation au $\mathrm{xxI}^{\mathrm{e}}$ siècle, pour lesquels des réponses innovantes devront être dégagées dans ces mêmes secteurs, notamment dans les pays en voie de développement et les pays à revenu intermédiaire.

Alors que les «pratiques optimales » deviennent obsolètes ou demandent à être adaptées à des environnements en mutation, les acteurs du développement doivent

engagements du G8 et du G20 à soutenir le développement agricole et la sécurité alimentaire en Afrique et qui analysent jusqu'à quel point ces engagements ont effectivement été tenus (www.africapartnershipforum.org). 
constamment mettre au point des nouvelles mesures qui soient efficaces. Pour un impact durable sur la pauvreté rurale, il convient de relever ces défis complexes, à facettes multiples, et de saisir les opportunités qui se présentent - ce qui exige d'être en mesure d'innover.

Dans un monde de plus en plus globalisé, l'innovation joue un rôle pivot dans le renforcement de la compétitivité et le développement des opportunités, en zone rurale comme dans le secteur de l'agriculture et de l'alimentation en général. Des études de cas provenant du continent africain ont montré que certaines conditions sont nécessaires pour faciliter l'accès à l'innovation : des approches participatives ; des partenariats entre les producteurs agricoles et leurs organisations, les fournisseurs de services agricoles, les organismes d'État et les acteurs du secteur privé ; la stimulation des synergies entre les sources formelles et informelles de connaissance et d'innovation ; l'accès au financement et au crédit ; un contexte institutionnel propice à l'apprentissage et à l'innovation ; et enfin des réseaux mondiaux encourageant le dialogue entre innovateurs (voir par exemple Hussein, 2001 ; Juma, 2010). Ces conditions réclament un environnement porteur, soutenu par des politiques appropriées et innovantes. Les Nations unies considèrent en effet que l'innovation et les politiques innovantes sont décisives pour la réalisation des objectifs du millénaire pour le développement et pour la réduction de la pauvreté, notamment en Afrique (Nations unies, 2010).

Les politiques d'appui à l'innovation, à la recherche ciblée, au partage actif des connaissances et aux systèmes performants de conseil agricole ne suffisent pas. Des politiques bien conçues sont essentielles pour pouvoir accroître la productivité, la profitabilité et la durabilité environnementale des systèmes agricoles et alimentaires - notamment en ce qui concerne l'agriculture familiale et les exploitants à petite échelle. Toutefois, ces politiques ont besoin d'être soutenues par un surcroît de ressources - publiques et privées, nationales et internationales - afin de susciter une véritable transformation agricole qui bénéficie à toute la fraction la plus pauvre des producteurs et des consommateurs, notamment en Afrique du Nord. Étant donné la gravité des problèmes économiques, sociaux, et politiques que doit surmonter la rive sud de la Méditerranée, il s'agit là désormais d'un impératif politique pour les gouvernements et les organisations régionales et internationales. Les gouvernements de la région ont un rôle vital à jouer, et il est de leur responsabilité de créer un cadre politique approprié qui encourage l'innovation dans le domaine de l'agriculture et de l'alimentation tout en veillant à ce que les innovations soient accessibles à tous.

À partir de cas concrets de politiques de promotion de l'innovation agricole, cette contribution présente les difficultés et les enseignements tirés de ces expériences. Ces exemples comprennent des expériences nationales et infra-nationales au Maroc, la mise en place d'un réseau régional de systèmes de recherche agricole couvrant le Proche-Orient et l'Afrique du Nord, et la collaboration de partenaires de développement dans le cadre de politiques et de stratégies en faveur de l'innovation (FIDA, AFD et OCDE). 


\section{- Les concepts clés de l'innovation et leurs implications en matière de politiques, de recherche et d'action}

La notion d'innovation, complexe et multidimensionnelle, a été abordée dans le cadre d'autres contributions au présent ouvrage ainsi que dans la littérature générale consacrée à l'innovation (voir par exemple Berdegué, 2005 ; Biggs, 2008 ; Hall, 2006 ; Hall et al., 2001 ; FIDA, 2007 ; Banque mondiale, 2006). Ici, l'innovation peut simplement être perçue comme la recherche d'une meilleure solution à un défi quelconque par l'introduction d'un changement. Ces défis peuvent être considérés comme des « problèmes » à surmonter, pour lesquels des solutions efficaces doivent être mises au point. La qualité de ces solutions est un aspect clé de l'innovation.

L'innovation peut être conçue non seulement comme un résultat, mais comme un processus, qui donne lieu à un apprentissage permanent. Un modèle simplifié de processus d'innovation comprend l'analyse des circonstances locales, la reconnaissance des problèmes spécifiques à résoudre, la description de la demande, l'élaboration d'une solution innovante, son expérimentation et son application sur le terrain. Les innovations utiles peuvent être diffusées, partagées, puis étendues à un nombre plus élevé d'acteurs, d'une part, et à une plus grande diversité de contextes, d'autre part. Le processus d'innovation s'opère par paliers ou de manière radicale. Dans le premier cas, les innovations apparaissent par exemple sous la forme de petites améliorations qui, ensemble, sont susceptibles d'apporter un certain progrès à la vie quotidienne des populations avec un degré d'incertitude faible. L'innovation plus radicale suppose la mise au point d'une solution nouvelle - associée à un degré plus élevé de risque et d'incertitude, étant donné qu'elle modifie les pratiques en vigueur ${ }^{3}$.

Les partenariats entre chercheurs, universités, fournisseurs de services agricoles, organisations de la société civile et secteur privé (y compris les producteurs) facilitent la génération de solutions plus efficaces et plus performantes aux défis spécifiques que rencontrent l'agriculture durable et le développement rural. Il est ici indispensable de disposer d'un environnement et d'un contexte politique propices, qui à la fois stimulent la formation de nouveaux partenariats et offrent aux divers acteurs un cadre qui leur permette de travailler conjointement et avec succès à promouvoir les innovations technologiques, institutionnelles et politiques.

Ainsi les politiques sont-elles une dimension à étudier de près lorsque l'on se penche sur la question de l'innovation pour le développement durable dans l'agroalimentaire. Une attention particulière doit être accordée aux rôles joués par les politiques nationales et infra-nationales et par les organisations régionales et multilatérales dans la promotion de l'innovation, de la collaboration et de la coopération pour soutenir les processus innovants.

3. Bien souvent, les techniques innovantes sont des pratiques qui sont nouvelles dans un contexte local particulier, plutôt qu'issues d'idées entièrement nouvelles, bien que leur mise au point puisse procéder d'une créativité et d'une innovation locales plus que d'un simple transfert de technologie (Berdegué, 2005). 
Lorsqu'il s'agit d'aborder les problèmes de politiques en matière d'innovation dans ce domaine, trois questions fondamentales se présentent :

- premièrement, quels sont les principaux moteurs et les principaux espaces de l'innovation agricole et rurale pour le développement durable aux niveaux national, régional et mondial?

- deuxièmement, quelles sont les institutions qui ont su transmettre et promouvoir avec succès l'apprentissage et l'innovation, et de quel soutien ont-elles besoin ?

- troisièmement, quels cadres de gouvernance faut-il pour pouvoir affecter les ressources et gérer concrètement la complexité au service de l'innovation (par exemple : approches participatives de l'apprentissage, politiques publiques, financement et évaluation de l'impact)?

La table ronde consacrée aux politiques, organisée dans le cadre de l'ISDA de Montpellier en 2010, a contribué à dégager certains attributs clés des politiques qui, parmi celles actuellement appliquées en région méditerranéenne, promeuvent avec succès l'innovation dans l'agriculture et l'alimentation.

\section{- Les cadres et stratégies internationaux pour favoriser l'innovation dans les sciences et les technologies ${ }^{4}$}

Les politiques en matière d'agriculture et de sécurité alimentaire ont une importance cruciale pour le développement mondial et reconnue, au plus haut niveau, par les dirigeants du G20, au vu de l'extrême volatilité des prix des denrées sur les marchés régionaux et mondiaux en 2008 et 2010 et de ses répercussions sur les aliments de base.

Une étude récente de l'OCDE a indiqué que le doublement des prix des produits agricoles n'avait pas été majoritairement dû à la spéculation des marchés. L'OCDE et la FAO prédisent par ailleurs dans leurs Perspectives agricoles que le cours des produits agricoles restera vraisemblablement élevé et poursuivra sa hausse dans la décennie à venir - quoique peut-être sans réitérer les pics de 2007-2008 qui ont vu quadrupler le prix des céréales de base. Le défi de l'agriculture et de la sécurité alimentaire se déploie ainsi à l'échelle de la planète, et discuter de la manière d'y faire face en encourageant l'innovation paraît parfaitement pertinent à ce moment précis. De fait, l'OCDE a déjà travaillé sur l'innovation, la sécurité alimentaire et l'agriculture, au cours de l'année 2010 et depuis, y compris en organisant une réunion d'experts en 2010 pour réfléchir sur les moyens de faire de l'agriculture un secteur à forte concentration d'expertise, et en réunissant des experts et des représentants des gouvernements autour de la question de la sécurité alimentaire et de la volatilité des prix dans le cadre des discussions du G20, et de la formulation de la nouvelle stratégie de développement de l'OCDE.

Il est admis que l'innovation doit faire partie de la solution aux défis du développement agricole, de la productivité et de la sécurité alimentaire. Des instances et

4. Le contenu de cette section s'appuie sur la contribution de M. Gang Zhang, administrateur principal de la direction de la science, de la technologie et de l'industrie de l'OCDE, Paris. 
organisations internationales clés ont par conséquent mis au point des cadres et des stratégies à l'échelle internationale pour stimuler l'innovation dans les domaines de la science, de la technologie et de l'agriculture. L'OCDE a ainsi mis en place sa propre stratégie pour l'innovation en 2010 (OCDE, 2010). Reconnaissant qu'il n'existe pas de « recette universelle » sur ce point, elle y recommande une série de principes en matière de politiques d'innovation applicables aux pays de l'OCDE aussi bien qu'aux pays en voie de développement.

Ces principes mettent notamment l'accent sur les points suivants :

- doter les individus des moyens nécessaires pour innover, par l'éducation et la formation pour l'acquisition de compétences et de savoir-faire ;

- créer des conditions-cadres politiques et financières propices à la libération de l'innovation, notamment des structures favorables à la concurrence, un accès au financement, l'instauration de droits de la propriété intellectuelle, etc. ;

- créer et mettre en application le savoir, par un investissement dans la recherche-développement qui facilite la circulation et l'utilisation des connaissances en stimulant la formation de regroupements et de réseaux;

- mobiliser l'innovation pour relever les défis mondiaux et sociaux en améliorant la coopération et les transferts scientifiques et technologiques à l'échelon international, y compris par la mise en place de mécanismes internationaux de financement de l'innovation et de partage des coûts associés à la résolution des défis mondiaux, au nombre desquels figure celui de la sécurité alimentaire et de l'agriculture ;

- améliorer la gouvernance et l'évaluation des politiques d'innovation, y compris afin d'assurer la cohérence de ces dernières, en faisant de l'innovation une composante centrale de l'action gouvernementale, avec une vigoureuse impulsion politique au plus haut niveau.

La recommandation de principes ne suffit pas toujours et les pays doivent pouvoir disposer d'une assistance supplémentaire pour la mise en œuvre. L'OCDE les aide à perfectionner leurs stratégies d'innovation nationales sur la base de ses rapports par pays concernant les politiques d'innovation. Dans ces rapports, cette organisation évalue la performance de chaque pays en matière d'innovation et émet des recommandations quant aux politiques susceptibles d'améliorer leur système national d'innovation. Des rapports ont été rédigés concernant non seulement ses pays membres, mais également le Chili, la Chine et quelques économies émergentes telles que l'Afrique du Sud. Pour ce qui est des pays en voie de développement, l'OCDE prépare actuellement des rapports pour la région sud-est asiatique, le Viet Nam (avec la Banque mondiale) et le Pérou (avec la Banque interaméricaine de développement).

L'OCDE a publié en août 2010, conjointement avec le Centre de recherches pour le développement international (CRDI, Canada), une étude qui explore de manière plus approfondie le programme d'innovation pour le développement (OCDECRDI, 2010). Cette étude examine le rôle de l'innovation dans les pays en voie de développement, notamment en Afrique. Elle souligne que l'innovation est un moteur de la croissance économique à long terme et analyse les systèmes d'innovation et leur application, le rôle capital des connaissances dans l'innovation au service du développement et l'importance de disposer d'études et de statistiques nationales officielles qui soient comparables en matière d'innovation. Elle insiste sur la 
nécessité d'inscrire l'innovation dans un plan de développement général et formule des recommandations pour promouvoir, tant dans le secteur formel que dans le secteur informel, des activités visant à transformer l'agriculture en une industrie axée sur le savoir apte à stimuler la croissance économique.

De plus, l'OCDE travaille sur une gouvernance favorisant une meilleure coopération multilatérale dans les sciences, la technologie et l'innovation en vue d'affronter les défis mondiaux, y compris ceux relevant de l'agriculture et de la sécurité alimentaire. Ce projet est nécessaire pour renforcer la contribution de la science, de la technologie et de l'innovation à la résolution des grandes problématiques qui se posent à l'échelle mondiale, telles que le changement climatique, l'agriculture et la sécurité alimentaire, la sécurité énergétique et la santé. Il se propose d'aborder la question de la gouvernance en analysant le besoin de nouvelles approches et en formulant des recommandations en matière de nouveaux mécanismes de gouvernance. Il se concentre sur quatre problématiques fondamentales de gouvernance qui concernent les politiques d'innovation : (i) l'élaboration d'un programme et la fixation des priorités, (ii) les mécanismes de financement, (iii) les options en matière de cadres institutionnels, et (iv) la concrétisation du savoir scientifique en véritables solutions. Ce travail proposera des recommandations en matière de principes et de pratiques optimales pour l'OCDE dans le domaine des politiques et des stratégies d'innovation, selon les cas.

De nombreux pays sont susceptibles de tirer avantage d'une participation aux initiatives internationales et régionales qui visent à s'attaquer aux défis communs touchant à l'agriculture. Dans la formulation des politiques nationales d'innovation, il convient de tenir compte de la coopération internationale et régionale, qui doit faire partie intégrante de ces politiques (voir encadré 9.1).

\section{Encadré 9.1. Un exemple de réseau interrégional pour stimuler l'innovation agricole ${ }^{5}$}

L'expérience de l'Association des instituts de recherche agricole du Proche-Orient et de l'Afrique du Nord (AARINENA, Association of Agricultural Research Institutions in the Near East and North Africa), réseau interrégional, apporte des enseignements utiles en matière de coopération et de stratégies nécessaires à l'échelon régional pour favoriser l'innovation agricole. Elle permet notamment de mieux éclairer les trois axes de travail qui nous intéressent.

Espaces d'innovation agricole et rurale

pour le développement durable à l'échelon régional

Les stratégies nationales qui ciblent les petits exploitants réclament une certaine adaptation des systèmes de recherche et d'innovation. Dans le même temps, les stratégies nationales de recherche doivent diriger leurs efforts vers des priorités stratégiques nationales dans ce secteur, afin de pouvoir stimuler l'innovation et

5. Le contenu de cette section s'inspire de la contribution du Prof. Osama Momtaz, coordinateur pour le réseau de Biotechnologie Agricole du réseau AARINENA et assistant directeur de recherche à l'AGERI-ARC, Égypte. Voir http://www.aarinena.org 
être en mesure de répondre aux défis plus généraux. Cela implique de renforcer les infrastructures nationales et les capacités des ressources humaines à évaluer le développement de l'agriculture durable. Il est crucial pour les systèmes nationaux de recherche agricole (SNRA) de s'intégrer aux communautés internationales de recherche-développement en agriculture par le biais des plates-formes régionales pour la coordination des politiques et de l'apprentissage : les uns et les autres ont en effet des intérêts en commun et tous doivent s'adapter aux évolutions des normes et des réglementations internationales (telles que les incessantes modifications des réglementations concernant la prévention des risques biotechnologiques, un domaine dans lequel la coopération régionale se développe). La consolidation de la coopération régionale entre les SNRA du Proche-Orient et de l'Afrique du Nord en matière de renforcement des capacités et de gestion des ressources humaines par l'apprentissage mutuel constitue un champ d'action important auquel les réseaux régionaux tels que l'AARINENA peuvent apporter une valeur ajoutée.

Le soutien nécessaire aux institutions

de recherche pour la promotion de l'apprentissage et de l'innovation

Les SNRA demeurent des acteurs clés du développement et de la promotion de l'innovation agricole. Ils ont été mis en place dans le but de répondre aux besoins nationaux et d'évaluer le développement de la recherche agricole dans les domaines de l'apprentissage, de l'innovation et de la recherche. Aujourd'hui, ils doivent accéder et s'intégrer aux dimensions techniques régionales et mondiales du développement agricole et rural ainsi qu'à la gestion des politiques afin d'être en mesure d'influer sur les échelons régionaux et mondiaux et d'interagir avec eux. Pour cela, il leur faut resserrer les liens avec les universités et les acteurs sur le terrain.

\section{Cadres de gouvernance nécessaires pour stimuler les systèmes d'innovation}

Des politiques nationales saines qui visent à promouvoir la mise au point et la diffusion de technologies agricoles, à faire appliquer les réglementations appropriées, à mettre en place des stratégies efficaces de gestion et de gestion des risques (par exemple en systématisant l'évaluation des risques et l'analyse des coûts et bénéfices) et à élaborer des stratégies de communication contribuent à stimuler l'évolution et l'application de la recherche et de l'innovation agricoles au service du développement durable. Ces stratégies nationales de développement des technologies et des innovations agricoles doivent être préparées dans le cadre de la stratégie générale de développement du pays et doivent être mises à exécution par les responsables de l'action gouvernementale.

Malgré des contextes et des défis très semblables, ces pays ont éprouvé des difficultés, dans le passé, à parvenir à un niveau de coopération qui permette de promouvoir l'innovation agricole et rurale pour un développement durable en région méditerranéenne. Dans le contexte des mutations rapides qui interviennent actuellement en Afrique du Nord sur le plan social et politique, il peut être espéré, une fois que les priorités nationales de réforme auront été prises en considération, que plus d'attention pourra être consacrée à approfondir la coopération régionale, indispensable à la réalisation des objectifs communs en matière de sécurité alimentaire et de développement agricole. 


\section{- Le Plan Vert Maroc, exemple d'une stratégie nationale d'innovation ${ }^{6}$}

Au Maroc, les zones rurales et le secteur agricole ont connu de profondes transformations au cours des cinquante dernières années. Ce secteur représente environ $19 \%$ du produit national brut, emploie 4 millions de personnes en zone rurale travaillant sur un total de 1,5 millions d'exploitations - 13,5 millions de personnes dépendant en outre en grande partie de l'agriculture pour leur subsistance - et assure la sécurité alimentaire de près de 30 millions de consommateurs dans le pays (ministère de l'Agriculture du Maroc, 2008, 2009). Toutefois, de sérieux obstacles au progrès sont apparus ces dernières années. Ces obstacles sont en partie dus aux difficultés rencontrées dans l'ajustement du secteur agricole domestique aux nouveaux défis économiques, sociaux et environnementaux, et également aux changements liés à la mondialisation et aux opportunités et aux contraintes engendrées par ce processus. L'agriculture a eu du mal à bien s'adapter à ces évolutions pour rester compétitive et pour optimiser sa contribution à la croissance économique nationale. Les défis à relever ont obligé le Maroc à réagir et à innover en investissant mieux dans les moteurs du progrès et en tirant le meilleur parti du potentiel et des ressources humaines du pays.

Le gouvernement considère l'agriculture comme le principal moteur de la croissance économique nationale pour la décennie en cours. Par ailleurs, il reconnaît l'existence des enjeux sociaux qui touchent les producteurs comme les consommateurs, ainsi que le fait que le développement agricole doit être durable et doit prendre en compte la rareté des ressources en eau et la nécessité d'une mise en valeur territoriale rationnelle. Ainsi l'innovation sociale, agricole et économique se trouve-t-elle au coeur de la stratégie agricole marocaine - le Plan Maroc Vert. Ce plan national de développement agricole, lancé en 2008, est considéré comme une stratégie innovante de développement national qui favorise les investissements dans le développement agricole au Maroc en tenant compte de l'importance économique et sociale de ce secteur. Le plan repose sur deux piliers : d'une part, la mise au point de produits agricoles à haute valeur ajoutée et à haute productivité (ciblant environ 400000 exploitants à travers jusqu'à 900 projets) et, d'autre part, le développement social et la réduction de la pauvreté (ciblant environ 600000 à 800000 exploitants par le biais de 400 projets). Afin de pouvoir mettre en ouvre ces actions, le Plan se donne pour objectif d'attirer de 150 à 170 milliards de dirhams marocains d'investissement dans le secteur sur 10 ans (2010-2020). Ce cas illustre comment les pays méditerranéens espèrent optimiser la contribution de leur secteur agricole au produit intérieur brut et au développement général de la région.

Le Plan Maroc Vert représente un bouleversement par rapport au modèle dominant de développement des années précédentes, selon lequel l'agriculture « moderne et efficace », d'une part, et l'agriculture «traditionnelle » marginalisée, d'autre part, représentaient deux manières opposées d'aborder le développement. Cet ancien modèle projetait une vision étriquée du développement agricole, qui inhibait le fort

6. Le contenu de cette section se fonde sur la contribution du Dr Mohamed Ait-Kadi, président du Conseil général du développement agricole (CGDA), Maroc. 
potentiel d'innovation porté par la richesse et la diversité du secteur, ne faisait que très peu participer les acteurs des filières et n'accordait qu'une attention minime à l'exploitation des opportunités de marché qui se présentaient. Cette approche se traduisait par une marginalisation des petites exploitations.

Le plan est édifié sur un réel changement de paradigme. Il s'articule autour de deux principes de base : (1) « l'agriculture pour tous » et (2) la promotion d'une agriculture diversifiée, à la fois socialement inclusive et « moderne ». Il s'appuie sur les fondements suivants :

- premièrement, la reconnaissance du fait que l'agriculture sera la principale source de croissance pour les 10-15 ans à venir ;

- deuxièmement, le regroupement des producteurs en une organisation en partenariat avec d'autres acteurs tels que l'État, des institutions de financement, des fournisseurs d'intrants et l'agro-industrie afin que les petits exploitants pauvres puissent bénéficier de meilleures marges de profit et de revenus plus élevés ;

- troisièmement, l'inclusion de tous les acteurs - de l'agriculture irriguée très productive à haute valeur ajoutée jusqu'aux petites exploitations communautaires et familiales des montagnes et des oasis ;

- quatrièmement, la nécessité d'attirer des investissements privés pour compléter les apports de l'État ;

- cinquièmement, une approche contractuelle de la mise en œuvre de jusqu'à 1500 projets concrets ;

- sixièmement, la préservation des ressources naturelles pour veiller à la durabilité des activités agricoles ;

- et septièmement, la restructuration du cadre sectoriel (refonte des politiques concernant le foncier, l'eau et la fiscalité, modernisation des marchés et amélioration de l'accès à ceux-ci, et restructuration du ministère afin de mieux accompagner, suivre et évaluer ces évolutions).

Pour répondre de manière inclusive aux défis et aux objectifs décrits ci-dessus pour ce secteur, les politiques et les stratégies agricoles nationales et infra-nationales doivent donner la priorité au renforcement des capacités des producteurs agricoles, en reconnaissant que l'innovation occupe une position centrale dans cette approche et cette stratégie. Promouvoir l'innovation recouvre ici notamment : prendre en compte la diversité, réformer les systèmes de recherche-développement agricoles en favorisant une meilleure interface entre les agriculteurs, les producteurs et les chercheurs, constituer des pôles régionaux («agropôles ») réunissant en un même lieu la recherche agronomique et les autres services agricoles de soutien à la commercialisation (y compris des services relatifs aux produits et au marketing ainsi que des opérateurs du secteur privé), créer des centres de ressources à l'attention des agriculteurs familiaux et exploitants à petite échelle susceptibles d'agir en tant que médiateurs ou «têtes de pont » pour les innovations, et faire élaborer des plans régionaux conjointement par les collectivités locales et par le gouvernement, avec la participation de l'ensemble des parties prenantes. Pour que ce plan puisse être appliqué, un certain nombre de réformes sont nécessaires.

Le Plan Maroc Vert a été mis au point afin d'apporter une solution concrète à des défis réels, et représente une rupture majeure avec le modèle de développement des cinq dernières décennies. Il fait appel à une nouvelle approche consultative, 
négociée et contractuelle, conçue pour appliquer la stratégie par le biais d'accords sur des objectifs négociés de production et de prix pour des produits et des filières spécifiques. Cette démarche a été appliquée verticalement, par chaîne d'approvisionnement, par l'intermédiaire de contrats concernant l'élaboration de produits prioritaires, négociés avec des représentants des organisations professionnelles, y compris de producteurs, au sein du sous-secteur. Elle a également été appliquée horizontalement, au niveau des acteurs du développement agricole local et territorial, au travers de contrats régionaux et locaux établis entre les acteurs de la filière et les autorités régionales et/ou locales. Ces deux types d'activités ont été soutenus par des initiatives horizontales, trans-sectorielles, en lien avec la gestion de l'eau et $\mathrm{du}$ foncier, la commercialisation et les politiques commerciales, le financement, et la recherche-développement.

Plusieurs enseignements quant aux stratégies et aux politiques nationales susceptibles de stimuler efficacement l'innovation agricole dans la région peuvent être tirés de cette initiative :

- l'importance des approches transactionnelles pour clarifier et rapprocher les rôles de l'État et des différents types d'acteurs et de producteurs du secteur dans le processus d'élaboration des politiques ;

- l'importance de la décentralisation - la gouvernance distributive et la démocratie locale faisant partie des politiques de promotion de l'innovation de sorte que des personnalités locales ayant connaissance d'innovations locales puissent émerger et prendre part au processus d'élaboration des politiques et des stratégies ;

- la nécessité de stimuler l'innovation institutionnelle ;

- la nécessité de disposer de financements (y compris du secteur privé et des banques) pour soutenir l'innovation et la mise en ouvre des politiques ;

- la nécessité d'asseoir le progrès effectif dans ce secteur sur un esprit d'entreprise dynamique.

\section{W Programmes pour stimuler l'innovation agricole à l'échelon local ${ }^{7}$}

Les aspects micro-locaux des processus d'innovation, organisationnelle et institutionnelle en particulier, doivent également être pris en compte dans les politiques nationales d'innovation. Des exemples provenant de programmes de développement mis en œuvre au Maroc avec le soutien de l'AFD, entre autres, apportent un éclairage instructif sur les leçons qui peuvent être tirées des initiatives locales et des dynamiques institutionnelles locales pour la formulation et l'application des cadres juridiques nationaux, tels que le Plan Maroc Vert. Une analyse de l'expérience acquise dans le cadre de projets de l'ENFI (École nationale forestière d'ingénieurs), de l'IAV (Institut agronomique et vétérinaire Hassan II), de l'ENA (École nationale d'administration de Rabat), de l'Université de Rabat, de l'IRD (Institut de recherche pour le développement), de l'AFD, du Cirad (Centre de coopération

7. Le contenu de cette section s'inspire de la contribution de Claude Torre (AFD) dans le cadre de la table ronde de l'ISDA. 
internationale en recherche agronomique pour le développement) et d'autres organismes travaillant sur les services de l'eau, les services agro-pastoraux et d'autres aspects des secteurs de l'eau et de l'agriculture au Maroc dans les années 1990, se révèle riche d'enseignements en ce qui concerne les politiques nationales en faveur de l'innovation. Ces expériences, bien que faisant appel à des approches différentes, se sont dans l'ensemble révélées positives pour ce qui est de la mobilisation des acteurs et de l'amélioration des techniques de gestion des ressources naturelles locales.

Le Haut commissariat au plan (HCP, 2007) a souligné les limites des anciennes politiques agricoles marocaines, liées à une approche excessivement interventionniste et technocratique qui ne laissait pas suffisamment de place à une appropriation par les acteurs ruraux. Deux mesures se sont avérées cruciales, à savoir une innovation institutionnelle s'appuyant sur les institutions et l'autonomie locales d'une part, et, d'autre part, la co-construction d'institutions durables en collaboration avec les acteurs locaux. De manière similaire, une évaluation de plusieurs programmes de développement rural intégré soutenus par la Banque mondiale a conclu à la nécessité d'une continuité du soutien financier et, pour ce faire, à celle d'une décentralisation réelle du pouvoir et des responsabilités.

L'étude consacrée à la gestion concertée des ressources naturelles (GCRN) - un rapport récapitulatif des leçons tirées d'une série de projets de développement rural - a montré que ces programmes se sont concentrés sur les formes traditionnelles de GCRN. La plus connue de ces dernières est l'agdal, un système de pâturage géré collectivement. Un programme a été mis en œuvre dans le but d'améliorer et de moderniser le fonctionnement de l'agdal par l'incorporation d'éléments tels qu'une formalisation par écrit des règles collectives, la légalisation des associations de développement local avec autonomie fonctionnelle, la mise en place de coopératives ethno-lignagères avec un fonctionnement plus démocratique et une participation plus active des jeunes et des femmes, des chartes de territoire, des contrats pour l'usage des ressources forestières et pastorales, et des systèmes de paiement pour les services environnementaux.

Plusieurs enseignements découlent de ces expériences, en particulier :

- il est important de prendre conscience des institutions, des droits et des règles qui existent localement, de les comprendre et de les intégrer à une législation ou à une politique nationale de promotion de l'agriculture durable, du développement rural et de l'innovation pour créer un cadre juridique « hybride » qui reflète les réalités locales et qui relie les politiques nationales aux plans locaux; la co-construction de politiques respectant l'autonomie locale est un facteur déterminant de la réussite des stratégies et des politiques nationales d'innovation efficaces et durables ;

- les acteurs et les institutions locaux sont à la base du succès des politiques de promotion de l'innovation dans l'agriculture et l'alimentation ; les politiques et les plans de développement touchant à un territoire géographique donné doivent reconnaître les institutions locales et les organisations ou coopératives de producteurs dans la loi ; l'inclusion de celles-ci implique en outre la nécessité de renforcer les capacités des acteurs locaux à mettre en œuvre et à modeler les politiques ;

- une approche spatiale ou territoriale de la mise en œuvre des politiques nationales de développement et d'innovation dans le monde rural facilite l'autonomisation et 
la coordination des différents acteurs, tels que les organisations locales, la société civile et les fournisseurs de services agricoles ;

- s'engager auprès des institutions locales et les renforcer est un processus lent qui réclame une approche progressive et à long terme de l'élaboration et de l'application des politiques. Les stratégies nationales doivent pouvoir prendre en compte et intégrer la diversité des acteurs et de leurs besoins ; la décentralisation doit être réelle et permettre aux collectivités locales de mobiliser des ressources financières pour soutenir ces institutions locales ;

- les innovations sont suscitées par l'instauration d'une législation « hybride » et d'une culture de contrats qui mettent en place des incitations économiques durables et négociées ;

- dans tous les cas, les stratégies et les plans nationaux d'innovation restent importants pour faciliter la cohérence entre les échelons locaux et nationaux de développement et d'innovation dans le secteur, d'une part, et, d'autre part, les défis surgis des contextes économique, environnemental et commercial internationaux.

\section{- Implications pour la promotion de l'innovation en Méditerranée}

Les études de cas analysées plus haut démontrent l'importance de politiques spécialement conçues pour promouvoir l'innovation dans le domaine de l'agriculture durable et de l'alimentation. Elles permettent par ailleurs de dégager un certain nombre d'enseignements concernant la mise au point et l'application de politiques pertinentes en la matière.

Les politiques d'innovation en Méditerranée doivent tenir compte des réalités politiques, sociales et géographiques, et au premier chef de la diversité des contextes, même au sein de l'unique « région » que constitue le pourtour méditerranéen. Cette région comporte en effet deux parties bien distinctes, la rive nord et la rive sud, qui affichent des caractéristiques contrastées quant aux contextes, aux niveaux de développement économique, aux ressources disponibles, aux contraintes subies et aux opportunités qui se présentent.

La rareté des ressources en eau, les contraintes foncières, le déficit alimentaire et les conflits concernant l'utilisation des terres constituent autant de défis communs qui réclament des innovations à l'échelle régionale. Il convient de réfléchir à ce qui peut être fait conjointement pour résoudre ces problèmes graves auxquels tous sont confrontés.

Dans le cadre de l'élaboration de politiques nationales visant à promouvoir une transformation radicale par rapport au passé afin d'être à la hauteur des nouveaux enjeux du développement durable dans le domaine agricole et alimentaire, il importe de trouver des moyens d'éviter de porter atteinte aux droits d'usage et d'accès aux ressources existants tout en prenant en considération les risques particuliers touchant aux femmes et à leurs droits.

Pour réussir, les politiques doivent tenir compte de l'expérience locale (encadré 9.2). Renforcer les capacités des acteurs est essentiel, tant au sein du gouvernement qu'à 
l'échelle locale, et l'expérience déjà acquise dans ce domaine précis révèle que la principale lacune à combler concerne l'aptitude du personnel à travailler sur le terrain, au niveau local, d'une manière efficace. Il s'agit là d'une compétence humaine subtile pour laquelle les formations officielles s'avèrent insuffisantes. Toutefois, il est important de s'assurer que l'État, par ses efforts dans ce sens, n'en vienne pas à évincer les initiatives locales et celles des exploitants agricoles eux-mêmes. L'innovation naît de l'interaction entre les politiques et les acteurs - un changement d'état d'esprit, de vision et de mentalité. Elle demande plus qu'une simple participation et exige de faire des compromis entre les intérêts et les acteurs.

\section{Encadré 9.2. Apprentissages pour la gestion participative de l'irrigation au Maroc Zakaria Kadiri, Marcel Kuper et Mostafa Errahj}

En 1995, une conférence nationale sur la nouvelle doctrine internationale de la «gestion participative de l'irrigation » a été co-organisée à Marrakech par le ministère de l'Agriculture et la Banque mondiale. Il s'agissait de mettre cette doctrine sur l'agenda des politiques nationales appelées à rendre effectif le désengagement de l'État par l'implication des associations d'irrigants dans la gestion des périmètres irrigués. C'est autour de cette innovation institutionnelle que l'État a mis en place deux périmètres irrigués dans le pré-Rif marocain : le Moyen Sebou en 1994 sur 6500 ha et Sahla en 2005 sur 3242 ha. Une deuxième tranche du projet Moyen Sebou est actuellement en construction sur 4600 ha.

De par sa configuration technique conçue pour une gestion centralisée par l'État, le périmètre du Moyen Sebou connaît les mêmes contraintes des grands périmètres en mode gravitaire : un accès à l'eau partagé entre plusieurs associations et suivant un tour d'eau, une difficulté de recouvrir les redevances, et des équipements hydrauliques complexes (stations de pompage, notamment) nécessitant des interventions lourdes et une expertise pas toujours disponible localement. L'implication des agriculteurs à travers des associations d'irrigants n'est intervenue que progressivement, souvent après la mise en place du périmètre. Malgré cette participation a posteriori, les associations d'irrigants se sont progressivement appropriées la gestion du périmètre. Elles ont embauché des jeunes de la région pour prendre en charge le fonctionnement du réseau. Elles ont établi de nouvelles règles concernant la gestion des tours d'eau pour faciliter l'irrigation de nuit, et soulager la demande en période de pointe en incitant les riverains de la rivière à irriguer par pompage privé. Elles ont aussi instauré des règles facilitant le recouvrement des redevances d'eau, par exemple en demandant aux irrigants de payer la facture d'eau avant irrigation, ou en constituant un référent unique en la personne du directeur technique pour échapper au clientélisme potentiel de certains membres des bureaux des associations. Certaines associations ont intégré de nouvelles parcelles dans le périmètre ou ont obtenu un accès direct à l'eau d'irrigation.

Le projet Sahla a été largement inspiré par l'expérience du Moyen Sebou, en partie parce que l'équipe responsable de la construction du périmètre était auparavant impliquée dans la mise en place du projet Moyen Sebou. Contrairement à ce dernier, à Sahla, l'équipe était installée sur le terrain et comprenait une équipe d'animateurs, jeunes diplômés de la zone, chargés de l'animation et de l'appui à la mise en place des associations d'irrigants. Conscients que la mise en valeur agricole n'avait pas été suffisamment réfléchie pendant la phase de construction du périmètre du Moyen Sebou, l'équipe a mis en place un programme de formations et réunions 
d'animations pour créer des organisations professionnelles agricoles et pas seulement des associations d'irrigants.

L'expérience du modèle Moyen Sebou-Sahla comme innovation institutionnelle montre un double processus d'apprentissage, à la fois par les agriculteurs et par l'administration. Les associations d'irrigants sont devenues progressivement des acteurs effectifs, même si cela n'est pas le cas pour toutes les associations où des échecs sont constatés. L'appropriation de l'innovation institutionnelle passe d'abord par la maîtrise de la gestion de l'aménagement à travers les associations d'irrigants tout en adaptant les règles de gestion, et ensuite par la capacité des agriculteurs leaders à investir d'autres sphères comme les OPA, le développement local ou la politique locale. Pour l'administration, cette innovation a constitué un véritable laboratoire, déléguer la gestion d'un nouveau périmètre aux agriculteurs ne suscite plus les mêmes craintes et les agents de l'État se sont habitués à être en négociation permanente avec les agriculteurs.

Enfin, notre propos montre que l'innovation institutionnelle n'est pas uniquement une « composante technique » pour gérer les périmètres irrigués, elle dépend aussi d'une volonté politique au niveau national pour « démocratiser » ces périmètres et des rapports de force entre groupes d'intérêts sur le terrain. Dans un contexte de transition, la mise en pratique de la gestion participative en irrigation ne se fait pas sans difficultés, et elle se fait concurrencer par d'autres modèles d'action. Les politiques nationales explorent ainsi d'autres innovations présentées comme plus efficaces. Il s'agit en particulier de la mise en place de partenariats public-privé dans la gestion des périmètres irrigués. La place des agriculteurs dans ces partenariats n'est pas évidente au premier abord. Est-ce que des associations d'irrigants pourront s'imposer comme partenaire à part entière dans de tels dispositifs ou se ferontelles marginaliser dans un entre-deux entre l'administration et sociétés privées ? (Kadiri et al., 2010)

Ainsi que l'ont puissamment démontré les bouleversements du « Printemps arabe » de 2011, les bureaucraties gouvernementales et les institutions de la région, y compris celles associées à la recherche, au développement et aux politiques agricoles, doivent revoir la manière avec laquelle elles opèrent et organiser leurs priorités de sorte à pouvoir répondre aux espérances et aux attentes des citoyens, tant ruraux qu'urbains. Mais l'ampleur effective des réformes dont elles sont capables pour satisfaire les demandes de la population reste incertaine.

Afin de rester pertinents, les systèmes de recherche-développement des pays de la Méditerranée, et notamment de sa rive sud, demandent à être remodelés pour pouvoir aborder un programme d'innovation radical et participatif, garantir une utilisation équitable des ressources naturelles, mettre au point des réponses appropriées aux défis agricoles liés à l'évolution du climat, et tirer les leçons des changements qui interviennent dans la base de ressources et dans le comportement des populations. Les organismes de recherche-développement se doivent d'accroître leur capacité à travailler en coopération étroite avec les institutions de développement et les institutions locales et acteurs locaux. En outre, la recherche a besoin du soutien et de l'engagement fermes de l'État, et de ressources suffisantes de la part de l'ensemble des parties prenantes, pour pouvoir générer des résultats utiles et opportuns (voir les exemples d'innovations institutionnelles dans l'encadré 9.3). 


\section{Encadré 9.3. Innovations institutionnelles de la nouvelle stratégie céréalière en Tunisie Raoudha Khaldi}

En 2007, la flambée des prix mondiaux des céréales a provoqué en Tunisie un doublement de sa facture d'achat qui a conduit l'État à dresser une nouvelle stratégie céréalière (2009-2013) basée sur la promotion des céréales irriguées, comme alternative technique aux rendements qui demeurent faibles et fluctuants. Ce choix politique devrait s'accompagner de changements au niveau du dispositif institutionnel pour mieux assurer le transfert des innovations techniques et améliorer la relation recherche-vulgarisation-profession.

La nouvelle stratégie politique a préconisé trois innovations institutionnelles principales :

- création en 2009 d'un Institut national des grandes cultures (INGC) dont le rôle est de valoriser les acquis scientifiques. L'accent est mis, en priorité, sur la recherche appliquée, la communication avec les agriculteurs et la vulgarisation. Les céréaliculteurs tireraient profit des expertises et savoir-faire de l'Institut directement ou par le biais des organisations professionnelles, groupements de développement et coopératives de services agricoles. La démarche s'appuie sur les contratsprogrammes ;

- mise en place de projets de recherche fédérés (PRF) dans le domaine des grandes cultures. Pour mieux assurer son rôle de coordination, l'Institution de la recherche et de l'enseignement supérieur agricole (IRESA) a mis en place depuis 1998 des Commissions de programmation et d'évaluation de la recherche agricole (CPERA) regroupant des représentants de la recherche, l'administration publique et la profession (agriculteurs, agro-industriels, distributeurs). La recherche est articulée autour de projets fédérateurs (PF) pluridisciplinaires et multi institutionnels sur des thèmes prioritaires préalablement arrêtés. L'objectif est de mettre fin aux interventions descendantes, ponctuelles et isolées et promouvoir la recherche-développement $(\mathrm{R} \& \mathrm{D})$ pour mieux répondre aux besoins des agriculteurs. Des conventions de R\&D ont été établies avec les structures de développement. (les Commissariats régionaux au développement agricole, les Offices de développement et les Groupements interprofessionnels). Dans le domaine des grandes cultures, une nouvelle génération de projets, appelés Projets de recherche fédérés (PRF) a été initiée pour la période (2009-2012) afin de combler les défaillances des premiers projets : priorités de recherche définies par grande zone, ouverture des équipes aux partenaires socio-économiques locaux (producteurs, ONG, décideurs politiques, institutions régionales, agro-industriels), travail en réseau et contribution au transfert des innovations ;

- association de la profession aux efforts de transfert des innovations. L'Union tunisienne de l'agriculture et de la Pêche (UTAP), organisation syndicale, a renforcé depuis 2008 ses liens avec la recherche en lançant un programme unique pour l'accompagnement des agriculteurs à travers des accords de partenariats.

En conclusion, la transition vers un système institutionnel efficace est encore difficile. Les innovations institutionnelles récentes pourraient améliorer les performances techniques des exploitations, mais des efforts sont à orienter vers une vraie démarche participative, une vulgarisation rapprochée et ciblée. Bien que la recherche soit sur la voie d'une meilleure programmation, le processus de promotion, développement et partage des innovations avec les acteurs reste à la charge de plusieurs institutions, rendant complexe la coordination et la mise au point d'un 
programme cohérent. La transition promue par la nouvelle stratégie politique devrait être accompagnée d'une mise en service de « conseillers agricoles », représentant la nouvelle forme de vulgarisation privée auprès des exploitants De tels conseillers existent depuis 1998, mais étaient principalement au service des grands producteurs. Pour permettre une action plus large, la mise en place de groupements de producteurs est encouragée à un niveau local, de type groupement de développement agricole, pour prendre en charge les services des conseillers et établir des partenariats pour la promotion de l'accès à l'innovation agricole qui n'est aujourd'hui qu'au profit des grands producteurs (Khaldi et al., 2010.)

Les décideurs considèrent souvent le concept d'《 innovation » comme trop vague ou trop général pour pouvoir être appliqué aux politiques nationales concernant l'agriculture durable et le développement rural. En effet, il convient d'être plus clair quant au rôle souhaitable du gouvernement en matière d'encouragement à l'innovation dans l'agriculture et l'alimentation. De plus, le contexte des politiques changeant constamment, il faut que les concepts et les approches utilisés pour promouvoir l'innovation intègrent, gèrent et s'adaptent à ces évolutions en cours au fil du temps.

On ne saurait dynamiser le développement local dans ce secteur sans prendre en considération les enjeux et les politiques de développement régionaux et mondiaux. Il n'existe pas de solution universelle : les politiques les mieux adaptées à la résolution de chaque problème particulier sont à établir au cas par cas.

En région méditerranéenne, les politiques concernant ce secteur sont souvent trop déconnectées des réalités et des priorités locales. Les visions et les politiques nationales doivent s'adapter à la diversité, aux contextes locaux, aux consommateurs, aux marchés et aux infrastructures en place - en un mot, elles doivent devenir plus « humaines » et plus cohérentes. Comment parvenir à introduire de tels changements dans l'élaboration des politiques est une question cruciale qui reste à résoudre.

L'agriculture et le secteur de l'alimentation sont confrontés à un environnement qui évolue très vite. Les pays méditerranéens doivent développer leurs capacités d'analyse des politiques afin d'être en mesure de comprendre ces changements et d'y répondre. Il serait utile de mettre sur pied un centre de réflexion régional pour la Méditerranée, créant l'espace et le potentiel nécessaires à un recours effectif au dialogue, au partage des connaissances et au renforcement des compétences dans la région, embrassant l'ensemble des pays méditerranéens d'Afrique du Nord, et en liaison avec ceux du sud de l'Europe.

\section{" Conclusion}

Favoriser l'innovation et l'accès équitable à cette dernière passe nécessairement par la mise en place de cadres politiques adaptés. Les organisations internationales de développement, telles que l'OCDE, l'IFAD, la Banque mondiale et d'autres, reconnaissent toutes aujourd'hui l'importance vitale de l'innovation et ont mis au 
point leurs propres stratégies en la matière. Il reste cependant que, dans le domaine de l'agriculture et de l'alimentation, l'innovation n'a pas la même signification pour tous. Bien souvent, les exploitations familiales, les producteurs à petite échelle et les consommateurs ne sont pas la préoccupation première de ces stratégies et politiques. Les politiques conçues pour promouvoir l'innovation doivent être souples et adaptées au contexte, à la diversité des acteurs (ce qui inclut les agriculteurs à faible revenu) et aux transformations sociales, économiques et politiques qui les affectent.

Ce chapitre a eu pour thème principal les politiques de promotion de l'innovation dans l'agriculture et l'alimentation en région méditerranéenne, et notamment en Afrique du Nord. Cette partie du monde a pris une importance singulière en 2011, avec les bouleversements politiques, économiques et sociaux qui y ont été observés. L'espoir s'est mêlé à l'appréhension et aux désillusions alors que des mouvements sociaux se sont faits jour dans plusieurs pays méditerranéens d'Afrique du Nord pour réclamer des changements radicaux, une meilleure représentation et l'équité.

Les pays du G8 ont lancé des initiatives concrètes et ont alloué des ressources significatives en faveur du développement économique et de la réforme de la gouvernance dans ces pays, par exemple par le biais du partenariat de Deauville entre le G8 et quatre pays du Moyen-Orient et d'Afrique du Nord, dont le Maroc, l'Égypte, et la Tunisie. Le mandat de la Banque européenne pour la reconstruction et le développement (BERD) a par ailleurs été étendu à l'Afrique du Nord, pour laquelle des ressources considérables ont été mobilisées afin de soutenir la réforme de la gouvernance et les investissements. Ces actions ont modifié le tableau général pour installer l'équité, l'emploi et l'inclusion économique et sociale dans la région en tête des priorités politiques nationales, régionales et internationales. L'agriculture demeurant un secteur clé de ces économies, et la stabilité des prix des denrées alimentaires étant décisive pour le maintien de la paix urbaine et rurale, on ne saurait trop insister sur l'importance cruciale de mettre en place des politiques dynamiques en matière d'innovation agricole.

De l'analyse des politiques, des stratégies et des réseaux de promotion de l'innovation et du développement durable dans l'agriculture et l'alimentation en Méditerranée, qui a été esquissée dans ce chapitre, nous pouvons tirer les conclusions ci-dessous, articulées autour des trois grandes questions formulées précédemment. En effet, ces trois axes de réflexion sont susceptibles de pouvoir servir de guide pour l'examen périodique des stratégies et des politiques d'innovation dans la région, dans un contexte politique, social et économique régional en mutation rapide.

\section{Moteurs et espaces d'innovation agricole et rurale pour un développement durable}

\section{Clarifier le concept d'innovation}

Les innovations sont des solutions à la fois nouvelles et meilleures pour trouver une issue à des défis ou obstacles concrets spécifiques de contextes ou d'acteurs 
particuliers. Les acteurs locaux, le secteur privé, les communautés et les États ont tous des rôles à jouer dans l'identification et la promotion de l'innovation. Dans le contexte des pays en voie de développement, il est utile de penser l'innovation comme une conversion de connaissances - formelles, informelles et provenant de quelque source que ce soit - en valeur. L'innovation n'est pas synonyme de pure invention ; c'est en grande partie un processus qui commence après le stade de l'invention proprement dite. Enfin, l'innovation implique un changement d'état d'esprit, une transformation ou une adaptation des approches et des pratiques en vigueur jusque là.

\section{Moteurs et espaces de l'innovation}

Les grands défis tels que la sécurité alimentaire nationale et régionale, la volatilité des cours des denrées alimentaires, la pression environnementale et le changement climatique sont les moteurs qui appellent l'innovation, tandis que les processus d'innovation s'opèrent dans les espaces physiques (zones géographiques, territoires, régions, etc.), dans les filières et chaînes de valeurs des produits, dans les environnements juridiques et dans les contextes écologiques et politiques mondiaux. Pour que les cadres politiques de l'innovation soient opérants, ils doivent prendre en compte ce complexe de moteurs et d'espaces.

\section{Institutions de promotion de l'apprentissage et l'innovation}

\section{Le rôle des institutions locales, des organisations des filières et des acteurs}

Les acteurs locaux, les entrepreneurs du secteur privé et les initiatives locales sont des rouages essentiels de l'innovation, car ce sont eux qui convertissent le savoir en valeur pour l'économie en général. Les innovations institutionnelles, depuis le niveau local jusqu'aux niveaux infra-national et national, sont déterminantes pour pouvoir être à la hauteur des enjeux du développement du secteur agricole et alimentaire.

\section{Le renforcement des capacités}

Il est capital de renforcer les capacités des acteurs à tous les niveaux des systèmes d'innovation, depuis le producteur le plus démuni jusqu'aux intervenants issus de l'agro-business, des organismes de recherche-développement, et des administrations de l'État concernées par la recherche et le développement agricoles. Il sera alors plus facile de faire en sorte que les innovations soient pertinentes et réellement mues par la demande, tout en veillant à ce que les cadres politiques soient suffisamment adaptables et souples pour répondre à une grande diversité de réalités et d'opportunités.

\section{L'analyse des politiques et l'examen par les pairs}

Les pays ont besoin de renforcer leurs capacités à conduire des analyses de politiques au niveau national et régional afin de pouvoir formuler des réponses appropriées aux 
défis émergents et de faire circuler l'expérience acquise au bénéfice de l'ensemble de leurs territoires et des autres pays de la région. Les réseaux, tels que le réseau AARINENA décrit plus haut, peuvent ici se révéler de précieux atouts. En outre, les politiques d'encouragement à l'innovation doivent s'appuyer sur une meilleure compréhension des interactions qui existent entre les différentes parties prenantes confrontées à un défi commun susceptible de réagir favorablement à un traitement innovant. Un groupe de réflexion pour la région méditerranéenne paraîtrait opportun à cette fin, tout comme le développement de réelles capacités de dialogue entre les divers pays de la région et entre les deux rives de la Méditerranée.

\section{L'apprentissage des politiques}

L'apprentissage par les pairs, les plates-formes coopératives internationales d'apprentissage (telles que celles des forums de l'OCDE consacrés aux techniques et aux politiques) et l'apprentissage sud-sud sont indispensables pour faire progresser les politiques. Il est essentiel de retenir les enseignements tirés des études de cas et de mettre au point les outils et le matériel appropriés pour encourager ce processus.

\section{Cadres de gouvernance pour gérer la complexité de l'innovation}

\section{Le rôle du gouvernement}

L'État n'est pas le seul intervenant - ni même l'acteur principal - dans les processus complexes d'innovation. Il joue néanmoins un rôle important en mettant en place des incitations, en créant un environnement qui encourage et qui récompense l'innovation, et en développant l'aptitude de la société à innover. Les gouvernements doivent créer les conditions, investir des ressources et introduire les incitations qui permettent de favoriser l'innovation chez des acteurs très divers. Pour ce faire, il devient pressant de réformer le secteur public et les fournisseurs de services dans la région. Ce rôle de soutien à l'innovation de l'État s'articule autour de quatre pôles : (i) identifier les défis auxquels est confronté le pays (ou la région) et pour lesquels de meilleures solutions sont requises, (ii) développer les compétences des personnes en matière de créativité et de gestion des innovations, (iii) établir et contribuer à établir des espaces et des processus au service de l'apprentissage et de l'innovation, où les risques liés à l'innovation sont mieux régulés et gérés, et où les succès sont rendus plus probables ou plus rapides, et (iv) mettre en place des incitations et aplanir les obstacles qui s'opposent à l'innovation. Il ressort de tout ceci que l'innovation doit figurer au nombre des grandes priorités des politiques publiques.

\section{L’intégration régionale et les plates-formes régionales}

Ainsi qu'il a été démontré dans plusieurs régions du monde, l'intégration régionale, l'ouverture et la régulation des marchés de biens, la technologie et la présence de main-d'œuvre expérimentée sont des facteurs susceptibles de favoriser des processus d'innovation plus rapides avec de bons résultats sur le plan de la productivité et du développement durable. Les plates-formes régionales sont cruciales pour la mise en 
commun des approches, des pratiques optimales et des innovations, ainsi que pour l'élaboration de cadres politiques coordonnés de promotion de l'innovation.

\section{L'équité sociale, la participation et l'accès équitable à l'innovation}

Les politiques de promotion de l'innovation doivent accorder suffisamment d'attention aux dynamiques sociales et technologiques de l'innovation si elles veulent réussir à long terme. Elles doivent de ce fait en premier lieu s'attaquer aux questions de l'équité sociale et de l'accès aux innovations, en reconnaissant que les processus d'innovation et d'apprentissage s'appuyant sur l'action collective sont susceptibles de passer à côté de certains acteurs et de certains membres des communautés. Il importe donc de veiller à ce que les agriculteurs à petite échelle et les exploitations familiales puissent avoir voix au chapitre et accéder à l'innovation, mais également à ce que les femmes et les jeunes des ménages ou des minorités installés sur des terres ingrates aient le même accès que les autres aux opportunités offertes par les innovations. Les systèmes d'innovation doivent être participatifs à tous les niveaux de prise de décision et de mise en œuvre, et doivent permettre à l'ensemble des parties prenantes de s'exprimer.

\section{Considérations finales}

L'expérience présentée dans ce chapitre permet de dégager sept spécificités communes aux politiques qui ont su effectivement promouvoir l'innovation et le développement durable dans l'agriculture et l'alimentation :

- il faut se doter, aux échelons national, régional et mondial, de cadres politiques et de stratégies de promotion de l'innovation qui soient à la fois appropriées et complémentaires ;

- les politiques doivent partir des acteurs, des contextes, des cultures et des processus de transformation et s'y adapter. Elles doivent être «co-construites » pour être efficaces et pertinentes ;

- les systèmes d'innovation doivent être participatifs et inclure toutes les parties prenantes des sociétés concernées ;

- de nombreux obstacles s'opposent à l'élaboration de politiques pertinentes en faveur du développement durable dans l'agriculture et l'alimentation, mais l'expérience montre que la mise au point, l'application et la surveillance des politiques doivent s'opérer de manière consultative et participative ;

- pour y parvenir, il est essentiel d'investir et de proposer des incitations en faveur du renforcement des capacités - tant à l'échelle locale qu'au niveau des institutions nationales de recherche-développement ;

- les priorités des systèmes de recherche-développement dans le secteur doivent évoluer et s'adapter pour se montrer à la hauteur des nouveaux défis et des nouvelles priorités qui se profilent aux niveaux local et national ;

- dans un environnement en mutation, les cadres politiques doivent être adaptables et flexibles. 


\section{" Références bibliographiques}

Banque mondiale, 2006. Enhancing agricultural innovation: How to go beyond the strengthening of research systems. Agricultural and Rural Development. WB, Washington, USA.

Berdegué J., 2005. Pro-poor innovation systems, FIDA, Rome, Italie.

Biggs S., 2008. Learning from the positive to reduce rural poverty and increase social justice: institutional innovations in agricultural and natural resources research and development. Experimental Agriculture, 44, 37-60.

CEA/ONU-OCDE, 2010-2011. Examen mutuel de l'efficacité du développement en Afrique. Paris, France, http://www.africapartnershipforum.org

FIDA, 2007. Stratégie du FIDA en matière d'innovation. FIDA, Rome, Italie.

Hall A.J., Sivamohan M.V.K., Clark N., Taylor S., Bockett G., 2001. Why Research Partnerships Really Matter: Innovation Theory, Institutional Arrangements and Implications for the Developing New Technology for the Poor. World Development, 29 (5), 783-797.

Hall A., 2006. Public private sector partnership in a system of agricultural innovations: concepts and challenges. International Journal of Technology Management and Sustainable Development, 5, 1.

HCP, 2007. Prospective Maroc 2030 - Agriculture 2030 : Quels avenirs pour le Maroc ? Haut Commissariat au Plan, Maroc.

Hussein K., 2001. Producer Organizations and Agricultural Technology in West Africa: Institutions that give farmers a voice. Development, 44 (4), 61-66.

Juma C., 2010. The New Harvest: Agricultural Innovation in Africa, Oxford University Press.

Kadiri Z., Belmoumene K., Kuper M., Faysse N., Tozy M., Errahj M., 2010. L'innovation institutionnelle dix ans plus tard : quelles opportunités pour les agriculteurs, et quels apprentissages pour les pouvoirs publics? Cas des associations d'irrigants au Nord du Maroc, In : Innovation and Sustainable Development in Agriculture and Food (ISDA), Montpellier, France - http://hal.archivesouvertes.fr/hal-00523316/en/

Khaldi R., Zied Dhraief M., Albouchi L., 2010. Innovations institutionnelles face à la crise pour une meilleure adoption des innovations techniques des céréales irriguées en Tunisie, In: Innovation and Sustainable Development in Agriculture and Food (ISDA), Montpellier, France - http://hal. archives-ouvertes.fr/hal-00522038

Ministère de l'Agriculture du Maroc, 2008. Plan Maroc Vert : Premières perspectives sur la stratégie agricole. Présentation PowerPoint en ligne.

Ministère de l'Agriculture du Maroc, 2009. Plan Maroc Vert. Présentation PowerPoint.

OCDE, 2010. La stratégie de l'OCDE pour l'innovation : Pour prendre une longueur d'avance, OCDE, Paris, France.

OCDE-CRDI, 2010. Innovation and the Development Agenda, OCDE, Paris, France.

OCDE-FAO, 2011. OECD-FAO Agricultural Outlook 2011-2020 http://www.agri-outlook.org/

ONU, 2010. Assessing Progress in Africa Toward the Millennium Development Goals. ONU, New York, USA. 


\title{
Chapitre 10 \\ Rôle d'une gouvernance redynamisée de l'agriculture africaine
}

\author{
Papa Abdoulaye SECK, \\ Aliou Diagne, Ibrahima BAmBA
}

L'année 2010 revêt pour l'Afrique une signification toute particulière. Elle marque pour la plupart de ses pays le cinquantième anniversaire de leur accession à la souveraineté internationale. À notre sens, une telle occasion doit aussi être saisie pour s'interroger sur les performances des agricultures.

Même si le secteur agricole connaît une croissance appréciable depuis le milieu des années 1990 (Badiane, 2008), sa performance depuis les années 1960 apparaît globalement insatisfaisante (Banque mondiale, 2007). Car pour certaines denrées alimentaires de première nécessité, le continent africain, malgré ses innombrables potentialités agricoles, connaît un déficit alimentaire préoccupant depuis plusieurs décennies.

Le secteur agricole en Afrique contribue à hauteur de $16 \%$ à la formation du PIB comparé à une moyenne d'environ 1 à $8 \%$ pour les autres continents. Il mobilise en moyenne près de $75 \%$ de la population, mais sa place dans les budgets publics reste faible. D'après la Banque mondiale (2007), la part des dépenses publiques dans l'agriculture africaine était inférieure à $4 \%$ en 2007. Malgré la prédominance de l'agriculture comme source de revenu et de moyens d'existence pour la majorité des populations africaines, le continent demeure un importateur net de produits alimentaires de première nécessité comme le riz et le maïs. Ainsi, l'Afrique abrite $15 \%$ de la population mondiale, mais absorbe, respectivement, $32 \%$ et $25 \%$ des importations mondiales de riz et de blé. Selon la FAO, près d'un Africain sur trois souffre de sous-alimentation en 2006 alors que dans l'ensemble du monde ce taux est estimé à $13 \%$.

Ce faible niveau de performance de l'agriculture africaine ne résulte pas entièrement de l'absence de politiques agricoles, mais elle est en partie imputable au sous-investissement dans ce secteur vital et surtout à l'insuffisance de la pertinence et de la cohérence des politiques agricoles par rapport aux véritables enjeux du présent et du futur. L'accroissement rapide de la population et son urbanisation à grande vitesse constituent deux défis majeurs pour l'agriculture africaine. En effet, l'Afrique a enregistré un taux d'accroissement annuel de sa population de $2,5 \%$ contre $1,2 \%$ pour l'ensemble du monde entre 2000 et 2005. Par ailleurs, l'Afrique dans son ensemble 
s'urbanise à grande vitesse. Actuellement, le taux d'urbanisation du continent est de $38 \%$. Il est estimé que le nombre d'africains vivant en ville atteindra $48 \%$ en 2030 (UN, 2006). Avec une urbanisation galopante et une forte croissance démographique, un des principaux enjeux présents et futurs pour l'agriculture africaine est d'augmenter durablement la productivité et la production agricole pour nourrir les populations africaines tout en préservant les ressources naturelles.

Pour mieux prendre en compte les enjeux de l'agriculture africaine et relever le défi actuel et futur de l'alimentation des populations africaines, une transformation qualitative de l'agriculture africaine s'avère nécessaire. Cette transformation passerait par une modification de son mode de gouvernance en passant d'une approche linéaire et descendante top-down de l'administration de l'économie rurale, où les politiques sont décidées par l'État sans participation des populations, à une économie délocalisée, «co-gérée » et «co-évaluée » par tous les acteurs. Ce mode de gestion rénovée de l'agriculture africaine nécessite le développement d'un environnement politique, économique et institutionnel stable, cohérent et équitable permettant aux différents acteurs des chaînes des valeurs agricoles d'adhérer aux orientations politiques conjointement définies et de nouer des relations commerciales durables et mutuellement bénéfiques. Le mode de conception novateur de la politique agricole proposé est fondé sur une gouvernance plus démocratique de ce secteur. Il implique l'adhésion et l'engagement effectif de tous les acteurs dans des processus transparents d'établissement des priorités et de prise de décision.

Dans ce chapitre, quelques propositions pour une réforme du mode de gouvernance de l'agriculture africaine seront présentées. Dans un premier temps, une brève et sélective étiologie des contre-performances de l'agriculture africaine est dressée. Puis, quelques dispositions et mesures pratiques à mettre en œuvre pour une transformation qualitative de l'agriculture africaine sont présentées.

\section{"Étiologie de la mauvaise prise en charge des questions agricoles}

L'augmentation de la production agricole est une condition nécessaire pour nourrir les populations africaines, mais elle est loin d'être suffisante. Un développement agricole durable nécessite une cohérence de l'ensemble des politiques de développement, notamment de la politique de développement agricole avec les politiques macroéconomiques, commerciales, d'industrialisation, de recherche et de développement, d'éducation et de formation, etc. Mais dans nombre de pays africains, les politiques agricoles étaient conçues comme si l'agriculture pouvait améliorer ses performances en faisant abstraction des autres composantes de la vie socioéconomique. À titre d'exemple, un large déséquilibre des comptes extérieurs, ou de la balance des paiements ou encore une forte instabilité du taux de change peut mettre à mal toute politique de relance du secteur agricole. Krueger et al. (1988) ont montré que les taxations implicites de l'agriculture découlant d'une surévaluation du taux de change ou des politiques de protection du secteur industriel avaient un impact sur les distorsions des prix agricoles qui dominait la taxation directe du secteur à travers les taxes levées sur les exportations. 
Par le passé, les États africains ont eu tendance à privilégier les approches productivistes qui consistaient à rechercher une augmentation des superficies cultivées, des rendements, et donc des productions agricoles sans trop se soucier de la rentabilité au niveau de tous les maillons de la chaîne de valeurs agricoles (Banque mondiale, 2007 ; Badiane, 1994). En d'autres termes, les États ont tenté de régler les problèmes à l'intérieur des champs et ignorer ceux qui sont à l'extérieur. Les signes d'échec repérables de cette approche sont nombreux. Il s'agit entre autres de la baisse des revenus ruraux dus à l'absence de circuits de commercialisation appropriés de la production locale et des coûts de transaction élevés dus au déficit d'infrastructure et des difficultés d'accès aux zones de consommation.

En outre, il était usuel de constater encore que des décideurs politiques déterminaient unilatéralement des spéculations prioritaires à promouvoir, les superficies à emblaver et les niveaux de production à atteindre sans aucune concertation avec les producteurs, les transformateurs et les autres parties prenantes. Cette façon d'opérer qui persiste encore dans une certaine mesure ne reconnaît aucune capacité d'analyse aux producteurs et aux opérateurs privés. Dans certains cas, les décideurs commandent des études qui vont s'appuyer sur des processus participatifs, mais sans associer les acteurs des filières agricoles aux processus d'établissement des priorités et de prise de décisions. Dans ce cas de figure, les acteurs sont consultés mais il n'y a pas encore de co-décision. Il faut aussi ajouter l'inefficacité des interventions étatiques liées à la non-prise en considération de l'innovation pouvant améliorer les performances des chaînes de valeur mais également la timidité des subventions et leur répartition sur des bases souvent subjectives parce que fondées sur des considérations politiques.

\section{Formation des acteurs et faible niveau d'organisation}

La formation et l'information des opérateurs des filières agricoles ne sont pas articulées autour de programmes durables et continus d'acquisition de nouvelles idées, connaissances et savoir-faire. De manière générale, elles sont liées à des projets, donc s'inscrivant dans un laps de temps généralement court. Sans formation et information adaptées, certains transferts de compétences ne produisent assurément pas les résultats escomptés.

Concernant les organisations professionnelles agricoles, elles souffrent du manque de moyens. En outre, elles n'ont pas toujours une philosophie et des principes de base garantissant une bonne gouvernance et une défense conséquente des intérêts professionnels.

La recherche agricole n'est pas toujours considérée comme un secteur essentiel pour stimuler une croissance agricole durable et continue. Or, les études sur la rentabilité de la recherche agricole révèlent qu'il est judicieux d'investir les fonds publics dans ce sous-secteur. Ces taux de rentabilité en Afrique sont généralement supérieurs à $30 \%$, voire nettement plus élevés (Alston et al., 2000). Par exemple, le taux de rentabilité interne moyen des investissements dans la recherche et la dissémination des «variétés Sahel » de riz dans la vallée du fleuve Sénégal est estimé à plus de $221 \%$ ce qui est largement supérieur au coût d'accès au capital, évalué à 
$18 \%$ sur la période 1995-2004 (Fisher et al., 2001). Ces taux de rentabilité élevés de la recherche agricole publique en Afrique exigent, en contrepartie, des investissements massifs dans ce secteur stratégique pour améliorer la performance de l'agriculture africaine.

Malgré des résultats prometteurs, la recherche agricole publique n'est pas suffisamment au cœur des préoccupations politiques. Souvent, des décideurs politiques séduits par les rendements obtenus avec certaines variétés développées à l'étranger décrètent leurs importations sans consulter préalablement les compétences nationales. Pourtant, les stratégies de transfert de technologies étrangères ont été tentées à maintes reprises par le passé et se sont généralement soldées par des échecs. La plupart des variétés de céréales importées des autres régions du monde en Afrique se sont révélées moins résistantes aux stress biotiques et abiotiques du continent, mais aussi fortement plus dépendantes aux engrais. Ainsi, l'introduction directe de variétés de sorgho et de mil en provenance d'Inde en Afrique de l'Ouest s'est soldée par un succès très limité (Matlon et Spencer, 1984). De même, sur plus de 2000 variétés de riz de mangroves importées d'Asie pour mener des essais en milieu africain, seules deux variétés avaient une performance comparable aux meilleures variétés locales (Matlon et Spencer, 1984).

Les résultats de la recherche agricole nationale ne sont pas souvent appliqués. Ceci s'explique en partie par l'absence de synergie entre la recherche et la vulgarisation agricole. Dépourvues de moyens financiers et techniques, la recherche et la vulgarisation agricole travaillent souvent de façon isolée. La formation des acteurs obéit aussi à une logique «techniciste » en lieu et place d'une approche plus globalisante intégrant les aspects techniques, économiques et commerciaux. Il s'agit d'une conséquence des approches productivistes.

\section{" Transformation qualitative de l'agriculture africaine}

La définition de politiques agricoles novatrices pour « produire plus et mieux » en Afrique nécessitera une rupture avec les approches dirigistes, productivistes et non systémiques pour mettre l'accent sur des approches plus participatives et décentralisées. Pour y parvenir, il s'avère nécessaire de réformer le mode de gouvernance de l'agriculture africaine qui passera d'une économie rurale administrée à une économie décentralisée, «co-gérée » et «co-évaluée » par tous les acteurs et par la mise en place d'un mode de gouvernance plus démocratique impliquant l'adhésion et l'engagement effectif de tous les acteurs dans des processus transparents d'établissement des priorités, de prise de décision et d'évaluation des impacts. Dans cet exposé, il est souligné que la transformation qualitative de l'agriculture africaine nécessitera une réforme de son système de gouvernance. Cette transformation qualitative de l'agriculture africaine suggère un partenariat élargi impliquant tous les acteurs intervenant dans le secteur.

Pour être effective et fonctionnelle, la réforme du mode de gouvernance de l'agriculture africaine doit veiller à : (i) mieux délimiter les domaines d'intervention et responsabilités opérationnelles des différents acteurs tout en les rapprochant ; (ii) promouvoir la spécialisation des acteurs suivant le principe des avantages 
comparatifs ; (iii) renforcer la synergie et la complémentarité des actions et interventions des acteurs opérant au même niveau de la chaîne de production de la valeur ajoutée agricole, mais dans des domaines disciplinaires dissemblables ; (iv) appliquer le principe de subsidiarité entre acteurs opérants dans le même domaine d'activité afin de maximiser l'utilisation des ressources limitées des États africains. Pour passer d'une approche consultative à une approche réellement participative, tous les acteurs doivent être formés et sensibilisés aux exigences de cette dernière. En particulier, des organisations professionnelles fortes et bien structurées doivent être promues afin qu'elles apportent une contribution active au processus d'élaboration, de mise en œuvre et d'évaluation des politiques agricoles. Par ailleurs, une formation polyvalente doit être offerte aux producteurs afin de mieux les outiller pour affronter convenablement la diversité et la complexité des problématiques de développement agricole.

\section{" Organisations professionnelles bien formées et informées}

À l'instar des autres domaines d'activités socioprofessionnels, le monde rural a aussi besoin de contre-pouvoir. Le regroupement d'acteurs ayant les mêmes intérêts dans une filière agricole constitue une condition nécessaire, mais pas suffisante. Ces organisations doivent davantage se professionnaliser en adoptant un certain nombre de principes. Parmi ceux-ci, on peut citer, sans être exhaustif : (1) vision partagée ; (2) statuts et règlement intérieur compris et respectés ; (3) tenue régulière des instances ; (4) prise en compte des questions de genre ; (5) tenue d'une comptabilité transparente et auditable ; (6) évaluation externe des activités ; (7) renouvellement des instances selon les dispositions retenues et limitation des mandats ; (8) libre circulation de l'information ; (9) reconnaissance de la légitimité des droits de chaque membre ; (10) reconnaissance et respect des obligations individuelles et collectives ; (11) adoption des décisions stratégiques par des instances qui se tiennent régulièrement ; (12) renforcement de la capacité des organisations professionnelles par des acteurs, choisies par appel d'offres quand l'appui est financé par l'État ou des projets.

Des progrès sont notés dans ce sens par les organisations professionnelles agricoles d'Afrique. Ainsi, l'option choisie par le Réseau des organisations paysannes et des producteurs agricoles de l'Afrique de l'Ouest (ROPPA) en 2008 de procéder à une évaluation externe de ses programmes d'activités pour la période 2000-2008 et la mise en place d'un dispositif consultatif de concertation par filière prioritaire (riz, horticulture, bétail-viande, lait et pêche) s'inscrit dans le cadre d'une recherche de perfectionnement de la gouvernance de cette association. Par ailleurs, le projet de fédération au niveau continental des organisations paysannes d'Afrique constitue une avancée majeure vers une meilleure structuration des organisations de producteurs. Toutefois, un soutien institutionnel fort est nécessaire pour renforcer le processus de professionnalisation des organisations professionnelles agricoles. Mais ce soutien institutionnel doit être défini avec les acteurs et non décrété ou imposé. 
Le renforcement des capacités des organisations professionnelles ne doit pas se limiter, comme c'est très souvent le cas, à des formations axées sur la production. Les acteurs du secteur agricole étant confrontés à une diversité de défis, les formations dispensées à ces derniers doivent refléter leurs préoccupations et attentes. En d'autres termes, les organisations professionnelles et les acteurs doivent être mieux outillés pour affronter convenablement la complexité de la réalité tant à l'intérieur qu'à l'extérieur de leur exploitation agricole.

\section{Mise en place d'observatoires des filières agricoles}

La qualité des politiques et stratégies de développement agricole est tributaire de la pertinence et la qualité des informations disponibles et de la qualité des mécanismes d'interactions entre les différents acteurs. Très souvent, en Afrique, l'information destinée aux organisations professionnelles et acteurs n'est pas fournie de manière continue et souvent n'est pas actualisée. Par ailleurs, elle est incomplète, car généralement elle se limite à la communication des prix à la consommation ou bord-champs enregistrés sur les différents marchés ou à l'estimation des quantités produites et des zones de production excédentaire ou déficitaire. Peu d'États acceptent ou ont les moyens de maintenir des systèmes d'information sur les marchés. Or la mise en place d'observatoires économiques de filière peut améliorer la collaboration et les interactions des différents acteurs.

Par observatoire économique d'une filière, il faut retenir un cadre d'échanges et d'analyse participative des problèmes par les différents intervenants de la chaîne de valeur. Au sein du cadre d'un observatoire de filière agricole, ce sont les représentants des catégories socioprofessionnelles qui sont chargés de collecter et disséminer des données et informations préalablement définies par les acteurs. Dans un observatoire, il convient d'avoir au moins les acteurs suivants : producteurs, transformateurs, distributeurs, consommateurs, exportateurs-importateurs, et représentants des services techniques de l'État impliqués dans le fonctionnement et la régulation de la filière (commerce, vulgarisation, recherche, etc.).

Un observatoire de filière agricole peut avoir pour objectif de développer le partenariat et d'instaurer une confiance mutuelle entre les acteurs afin d'améliorer la qualité de leurs interactions à travers des échanges d'informations. L'hypothèse implicite, à la base d'un observatoire, s'énonce ainsi : les utilisateurs des données d'une filière sont aussi détenteurs d'informations. Un observatoire de filière peut offrir un cadre d'apprentissage collectif et de renforcement des interactions entre acteurs à travers l'établissement de routines de travail, le développement de mécanismes endogènes permettant de surmonter des résistances, des oppositions, ou voire de résoudre des conflits. Dans un observatoire, chaque participant donne des informations sur le maillon de la filière qu'il occupe, et en reçoit des autres. La collaboration des acteurs de la filière au sein d'une observation peut contribuer à établir des relations de confiance et à l'émergence des partenariats constructifs entre les acteurs d'une même chaîne de valeur. À titre d'exemple : (1) les producteurs peuvent donner les prix bord-champs, les périodes de récolte ; (2) les distributeurs peuvent donner les prix de gros dans les marchés, les produits dont l'offre est déficitaire, les prix au 
détail, la qualité des produits ; (3) les exportateurs-importateurs peuvent donner les quantités exportées par spéculation, les tendances sur le marché international, etc. ; (4) les services de vulgarisation peuvent donner les variétés utilisées par espèces, etc. En fait, l'observatoire s'alimente à partir des informations fournies par les intervenants de la filière. Il est plus fiable qu'un système d'information classique car celui qui livre des informations le fait dans un cadre associant les acteurs avec qui il a eu des interactions et relations mutuellement bénéfiques.

\section{W Gouvernance de la recherche agricole}

L'approche linéaire et descendante de l'innovation agricole exclusivement générée dans les centres de recherche situés à une extrémité du pipeline de la production, des nouvelles connaissances jusqu'à l'adoption du nouveau produit, englobant les nouvelles connaissances à un autre bout du pipeline par les producteurs passifs, n'est plus de mise. En fait, il existe une pluralité de sources de connaissances et de technologies. Comme l'indique Hall (2009), l'innovation émane de l'intelligence collective mobilisée dans le cadre de la collaboration entre différentes sources de connaissance. À cet effet, l'innovation nécessite des connaissances provenant de sources multiples, y compris de la part des utilisateurs de ces connaissances (Hall, 2009). Ainsi, dans le processus d'adoption des nouvelles technologies agricoles, les producteurs jouent généralement un rôle d'adaptation des nouvelles technologies aux spécificités de leurs contextes. Avec la complexité et la diversité de l'environnement des agriculteurs pauvres, les technologies qui leur sont destinées nécessitent encore plus d'effort d'adaptation aux conditions locales (Douthwaite et al., 2001). Cette remarque s'applique particulièrement aux petits exploitants agricoles africains qui évoluent dans des environnements et conditions de production très hétérogènes. Par conséquent, une recherche agricole travaillant de façon isolée ne pourra pas seule être en mesure d'impulser une transformation qualitative de l'agriculture africaine. Par contre, la qualité et la diversité des interactions entre les producteurs agricoles, la recherche agricole, les services de vulgarisation et de conseil agricole, et les acteurs du secteur privé sont essentielles dans le processus de développement et de transformation de l'agriculture africaine, comme le montre l'encadré 10.1.

\section{Encadré 10.1. Plates-formes pour l'innovation dans le secteur de l'élevage en Éthiopie Kebebe Ergano}

Cet encadré présente les enseignements tirés des expériences du Fodder Adoption Project (FAP) mis en œuvre entre 2007 et 2010 par l'Institut international de recherche sur l'élevage (International Livestock Research Institute - ILRI) concernant la création et l'animation de plates-formes d'innovation de l'agriculture à petite échelle dans les pays en voie de développement. Le projet FAP a été conçu comme une initiative de « recherche-action » s'attaquant à divers enjeux du secteur de l'élevage en choisissant les problèmes d'alimentation du bétail comme point de départ. En Éthiopie, ses activités visaient principalement à mettre en place et à soutenir des réseaux de diffusion des technologies fourragères dans trois districts pilotes en 
faisant appel aux approches systémiques de l'innovation. Un des enseignements qui transparaît clairement est le fait que les divers acteurs rejoignent les plates-formes d'innovation lorsqu'ils y perçoivent des avantages matériels qui sont à la hauteur de leurs investissements en temps, en efforts et en ressources. L'existence d'avantages matériels, de préférence rapides, s'est avérée efficace pour gagner la confiance des agriculteurs et pour attirer l'attention de participants supplémentaires. Relier les technologies fourragères à un ensemble de problématiques propres à la filière dans les entreprises laitières et d'engraissement a été déterminant pour l'adoption réussie de ces technologies par les éleveurs. De même, la demande en technologies fourragères a été dynamisée par la prise en compte des autres problèmes généraux rencontrés par la filière laitière, tels que l'accès aux vaches laitières croisées, les services d'amélioration génétique et les services vétérinaires, le transport du lait et la mise en relation avec les marchés, le crédit, et l'accès aux moyens de production et aux informations. Des réseaux diversifiés d'acteurs sur l'ensemble de la filière se sont avérés nécessaires pour pouvoir traduire le savoir en actions et en bénéfices. De nombreuses tâches relevant de la gestion de l'innovation - telles que l'aménagement de l'accès aux techniques et aux marchés, l'organisation des producteurs, la formation, la construction de réseaux, la résolution des conflits, etc. - doivent être regroupées afin d'encourager l'adoption des techniques. Notre expérience a montré que la facilitation d'une plate-forme d'innovation joue un rôle central dans la production d'actions et de bénéfices à partir du savoir. Il faut pour le moins une personne, voire une équipe, pour endosser la responsabilité générale d'impulser le mouvement et de satisfaire les attentes que la plate-forme d'innovation fait naître chez les diverses parties prenantes. Les organisateurs et animateurs doivent se consacrer à entretenir l'énergie et la confiance chez les participants au fur et à mesure du déploiement de la plate-forme d'innovation. Cependant, dans les districts concernés, aucun organisme n'a été chargé de la facilitation des plates-formes ou ne s'est montré disposé à payer pour ce type de service. En effet, les décideurs ne reconnaissent pas suffisamment l'importance de favoriser les partenariats entre les diverses parties prenantes (chercheurs, agriculteurs, transformateurs, négociants, décideurs, organisations de la société civiles, etc.), et les conseillers agricoles conventionnels ne sont aucunement incités à assumer une fonction aussi lourde. Le système de conseil agricole conventionnel met plutôt l'accent sur l'apport technologique et l'approvisionnement en intrants. Le projet FAP a ainsi dû prendre lui-même en charge l'animation des plates-formes d'innovation et déléguer progressivement par la suite cette responsabilité aux organismes de conseil agricole des districts. Il peut en être déduit qu'investir uniquement dans le potentiel de recherche technique n'aura qu'un effet limité sur la promotion de la transformation agricole tant que ne seront pas développées les capacités d' « intermédiaires » dans les processus d'innovation. Doit également être renforcée la capacité à gérer la dynamique collective des configurations des organismes travaillant dans le cadre et autour d'un programme d'innovation. Un fonds spécial susceptible de couvrir les investissements expérimentaux potentiellement peu rentables et le coût des séminaires et des réunions ainsi que des installations supplémentaires destinées à la formation et au soutien serait en outre nécessaire. L'offre, aux organismes de recherche et aux universités, d'allocations concurrentielles en faveur de recherches-actions porteuses d'innovations systémiques pourrait largement contribuer à la création de champions de l'innovation. Cela permettrait en outre d'introduire des modifications dans les programmes d'enseignement et de faire émerger une génération de professionnels convaincus du bien-fondé de la pensée systémique de l'innovation (Ergano et al., 2010). 
La recherche agricole a aussi un rôle à jouer dans l'impulsion et l'émergence des interactions et des possibilités d'apprentissage interactif entre les différents acteurs intervenant dans la chaîne de valeurs agricoles. À travers le Challenge programme pour l'Afrique subsaharienne (SSA-CP), le FARA facilite la mise en ouvre d'une approche novatrice de promotion d'un processus d'innovation à travers l'interaction permanente entre les acteurs, l'utilisation du feedback issu des différents acteurs, l'analyse et l'intégration des leçons tirées de différents processus (Hawkins et al., 2009). Cette approche admet explicitement que l'innovation agricole ne démarre pas toujours avec la recherche agricole mais nécessite l'agrégation de la contribution de tous les acteurs y compris les producteurs agricoles. En tant qu'un des éléments constitutifs du système d'innovation, une des contributions de la recherche agricole est de répondre à la demande de connaissances et d'informations, de compléter les flux de connaissances existants plutôt que de les remplacer (Hawkins et al., 2009).

Nonobstant les énormes retombées de la recherche agricole et de son potentiel de contribution à la croissance, ce sous-secteur n'est toujours pas au cœur des préoccupations des décideurs politiques. Pendant les années 1990, les investissements publics en R\&D agricole ont diminué à un taux annuel moyen de 0,2\% (Beintema et Stads, 2010). De plus, les financements de la recherche et du développement agricole des pays africains étaient fortement dépendants des donateurs étrangers. En outre, un des principaux problèmes auquel font face les systèmes nationaux de recherche et de développement agricole est le vieillissement des chercheurs qualifiés. Le personnel partant en retraite n'a souvent pas été remplacé en raison de l'interdiction de recrutement frappant le secteur public dans plusieurs pays durant la période des programmes d'ajustement structurel. Malgré les retombées positives de la recherche agricole en Afrique, les investissements publics restent faibles, voire même baisse dans un grand nombre de pays, alors que les capacités scientifiques de l'Afrique demeurent faibles, car le continent ne contribue que pour $0,3 \%$ au capital des résultats scientifiques du monde et ne compte que 70 chercheurs pour 1 million d'habitants contre 4380 pour le Japon.

Pour maximiser la contribution de la recherche agricole au processus de transformation qualitative de l'agriculture africaine, les états africains doivent augmenter substantiellement les dotations budgétaires à la recherche agricole. À ce titre, la Conférence mondiale sur la recherche agronomique pour le développement (GCARD) organisé en 2010 a recommandé que les pays en voie de développement œuvrent pour un financement endogène de la recherche agricole en consacrant au moins $1,5 \%$ de leur PIB à ce sous-secteur stratégique. En plus, une réforme du mode de gouvernance de la recherche agricole en Afrique est également préconisée. Cette réforme concernerait une amélioration de la coordination et de la coopération entre les systèmes nationaux et régionaux de recherche agricole suivant les principes de subsidiarité et de complémentarité et en exploitant les avantages comparatifs des institutions existantes. Par ailleurs, cette réforme devrait, d'une part, favoriser la participation effective des producteurs, du secteur privé et la société civile dans les organes de gouvernance des centres de recherche, d'autre part, redéfinir les relations entre les chercheurs et les autres catégories professionnelles dans le cadre d'un nouveau partenariat basé sur une concertation dans la planification, l'exécution, l'évaluation et la valorisation des résultats de recherche. 


\section{" Les systèmes nationaux de recherche agronomique}

La conception et la mise en œuvre des programmes spécifiques de recherche pour le développement agricole doivent être principalement dévolues aux institutions spécialisées qui possèdent un avantage comparatif dans le domaine en question suivant le principe de subsidiarité tant géographique que thématique. Ainsi, suivant ce principe de subsidiarité, les systèmes nationaux de recherche agricoles (SNRA) doivent piloter toutes les activités de recherche pour le développement agricole qui se déroulent dans leurs pays, appuyés, si besoin, par les institutions régionales et internationales de recherche. Cet appui qu'apportent les institutions régionales et internationales de recherche aux SNRA (qui incluent aussi bien les centres nationaux de recherche que les universités) doit s'inscrire dans une optique de renforcement des capacités de recherche des nationaux pour leur permettre d'assumer à terme leurs responsabilités. Les interventions qui utilisent des raccourcis en substituant l'expertise nationale à l'expertise régionale ou internationale doivent être bannies, car elles ne font que perpétuer un système de dépendance et retarder le développement institutionnel et humain des SNRA.

\section{- Les dispositifs régionaux de coordination des recherches}

$\mathrm{Au}$ plan régional, la coordination et la mise en ouvre des programmes et projets de recherche dans un domaine ou filière agricole bien définie doit concerner en premier lieu l'institution de recherche régionale spécialisée disposant d'une expertise avérée dans ce domaine si elle existe ou, à défaut, l'une des organisations de recherche faîtières sous régionales (CORAF, l'ASARECA et le SACCAR) et régionales (FARA). Les organisations régionales et sous régionales de recherches spécialisées couvrent à la fois les organisations intergouvernementales et les centres du groupe consultatif pour la recherche agricole internationale (GCRAI) intervenant en Afrique. Par définition, un projet de recherche à caractère régional est un projet dont soit la thématique est purement régionale, soit sa mise en œuvre ou l'applicabilité des résultats attendus couvrent plusieurs pays. Par exemple, la coordination régionale assure la mise en œuvre des activités à caractère régional telles que les échanges régionaux, les travaux dans les laboratoires de pointe, les travaux d'appui méthodologique, etc. Les activités de nature nationale (enquêtes, essais, échanges locaux, etc.) seront prises en charge par la partie nationale. Tout projet qui ne répond pas à l'un de ces trois critères doit être dévolu au SNRA du pays concerné. Dans le cas où ce dernier ne possède pas toute l'expertise nécessaire pour la mise en œuvre du projet, un appui technique d'une institution régionale, internationale, ou d'un autre pays (développé ou sous-développé) doit être cherché dans le cadre de la coopération nord-sud ou sud-sud. Cependant, cet appui technique doit être conçu dans l'optique de développer l'expertise locale dans le domaine considéré.

Cependant, pour jouer pleinement leur rôle les institutions régionales doivent aller au-delà de ce que font les SNRA au niveau national en apportant techniquement une valeur ajoutée à l'agrégation des efforts des SNRA impliqués. La fonction de 
coordination à elle seule ne peut pas justifier l'implication d'une institution régionale dans la mise en œuvre d'un projet de recherche à caractère régional. Il faut que l'institution régionale puisse : 1) apporter une plus-value scientifique aux SNRA impliqués ; 2) faire un vrai travail de transfert de connaissance et de renforcement des capacités de recherche des SNRA ; 3) faciliter l'apprentissage et le partage d'expérience entre pays ; 4) assurer la qualité de la recherche par l'organisation de revues critiques par les pairs ; 5) organiser le partage des résultats de recherche avec les pays non impliqués dans l'exécution dudit projet ; et 6) faire un travail supplémentaire d'analyse, de synthèse et de communication au niveau sous-régional ou continental. Il est évident que pour que l'apport de cette valeur ajoutée soit possible et efficace, il faut que l'institution régionale puisse posséder en son sein une masse critique de chercheurs ayant l'expertise technique avérée dans le domaine considéré. Cette exigence n'est en général remplie que par les centres de recherche sous-régionaux ou continentaux spécialisés dans des domaines précis.

Par contre, les organisations de recherche faîtières sous-régionales et régionales (FARA, CORAF, ASARECA et SACCAR) disposent d'un avantage comparatif dans la coordination des programmes de recherche et de développement agricole qui ciblent plusieurs spéculations agricoles ou qui portent sur des thématiques transversales. Les organisations faitières régionales et continentales de recherche ont aussi un rôle très important à jouer dans le développement institutionnel des SNRA en promouvant l'adoption des meilleures pratiques de gouvernance par ces derniers tout en les aidant à maintenir une autonomie par rapport aux décideurs politiques en ce qui concerne leurs modes de gestion. En effet, des changements abrupts d'orientations et politiques dans leur gouvernance sont impulsés souvent par les décideurs politiques sans consulter véritablement les acteurs de la recherche, sans préparation adéquate et sans tenir compte des priorités et engagements définis antérieurement au niveau régional et national. Aucune organisation autre que FARA et ses démembrements sous régionaux est mieux placé pour jouer ce rôle, car ayant le degré d'indépendance et la légitimité nécessaire. L'amélioration de la gouvernance des SNRA est un vaste chantier qui lui seul peut occuper une grande partie de l'action du FARA et de ses démembrements régionaux pour bien des années à venir.

Une telle délimitation des domaines de compétence dans la coordination et la mise en œuvre des programmes de recherche pour le développement agricole permettra non seulement un usage à bon escient des ressources limitées, mais aussi contribuera à renforcer les capacités des institutions nationales et régionales de recherche tout en réduisant la duplication des efforts.

\section{- Pour une programmation concertée des priorités de recherche}

L'approche participative préconisée exigera la promotion d'une participation réelle des acteurs dans les organes de gouvernance des centres de recherche agricole, à savoir les producteurs, le secteur privé et la société civile. Étant les principaux utilisateurs de la recherche agricoles, ces acteurs peuvent veiller à la définition et mise en œuvre des priorités de recherche consignées dans un cahier de charges. Les 
acteurs du monde agricole doivent également participer à l'évaluation des activités de recherche et contribuer au plaidoyer de la recherche.

Par ailleurs, l'utilisation de l'expérimentation en milieu paysan au sein de la RAP est à l'origine des processus d'innovations et d'apprentissage et participe à la production

Encadré 10.2. Recherche-action-en-partenariat, une démarche de réconciliation entre recherche et société

\section{Mélanie Blanchard, Eduardo Chia, Mahamoudou Koutou et Eric Vall}

Dans les démarches de recherche-développement du secteur rural, la faible implication des acteurs de terrain à l'élaboration de pratiques innovantes limite la prise en compte de leurs besoins et de leurs contraintes dans la conception des innovations. En conséquence, de nombreux résultats de la recherche agronomique ont été peu ou pas adoptés par ces derniers.

La participation des acteurs de terrain à la définition des questions, à la recherche de solutions, à la mise en place des dispositifs puis à la mise en œuvre et à l'évaluation de ces solutions n'est expérimentée que depuis peu dans le domaine agricole. Des postures de recherches nouvelles, parmi lesquelles nous trouvons la Recherche-action-en-partenariat (RAP, Chia, 2004 ; Faure et al., 2010), invitent à repenser le rôle et la place de l'expérimentation agronomique ainsi que la relation entre les acteurs de terrain (producteurs, conseillers, techniciens représentants des services déconcentrés de l'État, ONG) et les scientifiques (Vall et al., 2008; Anadon et Sebillote, 2007). Cette posture originale a été expérimentée dans les projets de recherche-action Teria puis Fertipartenaires mis en œuvre dans l'Ouest du Burkina Faso. Ces projets, par un partenariat construit entre agriculteurs, techniciens et chercheurs, visaient la co-conception d'innovations agropastorales pour relever la fertilité des sols.

La démarche proposée a permis la concertation, le diagnostic de la problématique, l'exploration des solutions possibles et l'action par un partenariat construit entre acteurs de terrain et scientifiques avec une problématique partagée et une volonté de changement. Il s'agissait en particulier de co-concevoir des innovations sur la production de fumure organique (fosses à compost au champ, fosses fumières à la concession, compostage des tiges de cotonnier), les systèmes de culture innovants renforçant la place des légumineuses dans l'assolement (cultures associées céréales légumineuses, culture fourragère, culture sous couverture végétale) et l'élaboration d'une charte foncière locale de gestion des ressources naturelles.

Pour organiser le partenariat entre les acteurs de terrain et les scientifiques, deux innovations majeures ont été co-conçues dans le cadre de ces projets. Il s'agit tout d'abord du Comité de concertation villageois (CCV), composé d'agriculteurs, éleveurs, femmes, conseillers techniques,... et fonctionnant à partir d'un cadre éthique négocié. Le CCV est un lieu de décision, de partage de l'information et l'interlocuteur privilégié de la recherche, garantissant la participation effective des acteurs de terrain à chaque étape de la recherche (Koutou et al., 2010). La deuxième innovation étant le cahier des charges ou contrat qui est formalisé entre les acteurs de terrain participants au programme (via le CCV) et les chercheurs sur leurs engagements (qui fait quoi ?) pour l'expérimentation (Chia et Deffontaines, 1999 ; Chia, 2004, Chia et al., 2008, Chia et al., 2009 ; Blanchard et al., 2010 ; Koutou et Vall, 2010). 
des nouveaux savoirs locaux nécessaires à la co-production des innovations (Blanchard et al., 2010). Ces savoirs et apprentissages constituent une garantie de la durabilité.

Concernant la planification partagée de la recherche agricole, les chercheurs et les utilisateurs doivent mener une réflexion concertée sur : (i) les particularités de la filière en question et les principales contraintes auxquelles font face les producteurs ; (ii) la formulation des thèmes de recherche ; (iii) l'établissement des priorités entre les thèmes ; (iv) la stratégie globale de mise en œuvre de la recherche ; (v) l'impact potentiel attendu des recherches ; (vi) la définition des études et réflexions prospectives ; (vii) le bilan de la recherche. L'exercice est réussi si le cahier de charges de la recherche devient véritablement « la propriété de tous ». Les critiques relatives à la pertinence des enjeux stratégiques de la recherche sont de natures constructives.

La réforme préconisée de la gouvernance de la recherche agricole impliquera entre autres des changements touchant les critères d'évaluation des chercheurs et le comportement et l'attitude de ces derniers vis-à-vis de leur environnement. Le cadre d'une planification partagée des priorités de recherche doit favoriser l'émergence d'un chercheur de type nouveau qui saura d'une part, lire les messages des utilisateurs et les décoder en thématiques de recherche et d'autre part, communiquer avec les différentes catégories d'acteurs et mieux anticiper les conséquences sociales, politiques, économiques et environnementales des différents évènements. Par ailleurs, l'évaluation du « chercheur total » doit se fonder non seulement sur sa productivité scientifique, mais surtout sur : (i) l'impact de ses résultats sur le secteur agricole ; (ii) son aptitude à assumer des problématiques avec d'autres spécialistes ; (iii) les relations qu'il réussira à développer avec les partenaires nationaux, régionaux et internationaux; (iv) la valorisation des résultats de ses recherches.

\section{" Repenser le rôle de l'État dans le secteur agricole}

L'État doit se considérer comme un acteur parmi tant d'autres. Cela signifie s'ouvrir aux autres acteurs des chaînes de valeur à travers une approche participative et interactive. En plus de ses fonctions de régulation et de redistribution en faveur de l'intérêt général, l'État ${ }^{1}$ doit être un facilitateur ayant pleine conscience des enjeux actuels et futurs de l'agriculture et des objectives et intérêts quelquefois contradictoires des acteurs. Vu que le marché n'est pas en mesure de stimuler suffisamment l'innovation agricole, l'État a un rôle central à jouer dans la mise en place d'un environnement propice à l'émergence d'une diversité de types d'interactions et des possibilités d'apprentissages interactifs entre les différents acteurs intervenant dans la chaine de valeurs agricoles (Hall, 2009). Par conséquent, l'État doit promouvoir le développement d'institutions stables et de mécanismes institutionnels qui auront pour mission d'améliorer la qualité des mécanismes et processus de négociations, d'interactions, de concertations, de co-decison, co-gestion et co-évaluation

1. En tant que gardien de l'intérêt général, l'État peut définir des règles qui s'imposent à tous (sanitaires, sociales, etc.) et aussi légitimement définir les priorités nationales 
avec les organisations de producteurs et les opérateurs privés afin de renforcer les performances des filières agricoles.

Par ailleurs, il est nécessaire de toujours arrimer les interventions et politiques agricoles publiques dans le cadre global de la stratégie de développement et de transformation de la société. Les différents compartiments de la vie socio-économique ont des effets sur l'agriculture et ceux-ci ne relèvent pas nécessairement du ministère en charge de ce secteur. Il faut mettre dans ce registre, la gestion des marchés, les infrastructures de base, l'application des réglementations agricoles, les importations de produits agricoles, etc. Par conséquent, une transformation de l'agriculture exige une implication effective aussi bien des acteurs du secteur agricole que des intervenants évoluant dans les activités connexes.

Les services publics ont un rôle primordial à jouer dans l'amélioration de l'environnement de la production à travers des mesures réglementaires et des investissements publics appropriés, car définies en concertation avec les acteurs. Il apparait nécessaire de doter le secteur agricole d'un budget conséquent. En 2000 à Maputo, les chefs d'État avaient pris la résolution d'allouer au moins $10 \%$ du budget de chaque État à l'agriculture. Force est de constater, six ans après la résolution de Maputo, que seuls 10 pays africains sur 53 ont honoré leur engagement. En 2007, les pays africains n'ont consacré en moyenne que $4 \%$ de leur budget à l'agriculture.

Au-delà de la nécessité d'augmenter l'investissement public dans le secteur agricole, il est tout aussi impératif d'améliorer la qualité des dépenses publiques dans ce secteur stratégique en les alignant sur les priorités définies en concertation avec tous les acteurs de manière objective et transparente, en formant mieux les acteurs et en renforçant les institutions. Par ailleurs, l'amélioration de la qualité des dépenses publiques exige que les orientations et les prises de décision pour les investissements publics soient fondées sur des faits empiriques à travers une utilisation rigoureuse des résultats et données scientifiques. À cet effet, des résultats de recherche tendent à montrer que les subventions sur les biens et services privés comme les engrais et crédits sont moins efficaces que les investissements dans les biens publics de base comme la recherche agricole et les infrastructures rurales (Lopez et Galinato, 2007).

Toutefois, avec l'omniprésence des défaillances de marché (Omamo et Farrington, 2004), le recours aux subventions ciblées en direction de certains acteurs définis par rapport aux objectives de sécurité alimentaire, de redistribution sociale ou conservation des ressources naturelles, est économiquement fondé. La Banque mondiale (2007) reconnait que des subventions peuvent se justifier de manière temporaire pour favoriser l'utilisation des semences et des engrais même si l'objectif à long terme doit être l'émergence de marchés d'intrants viables qui reposent sur le secteur privé. L'Afrique étant le continent ou le niveau d'utilisation d'engrais minéral est le plus bas au monde avec en moyenne $13 \mathrm{~kg} / \mathrm{ha}$ d'engrais en 2008 contre $94 \mathrm{~kg} / \mathrm{ha}$ pour l'ensemble des pays en voie de développement (Minot et Benson, 2009), une hausse significative des rendements agricoles des petits paysans est techniquement peu probable sans une politique de promotion de l'accès aux engrais minéraux. Ce faible niveau d'utilisation d'engrais induit non seulement une faible productivité agricole, mais est responsable aussi de l'épuisement des sols. En effet, sans apport conséquent de fertilisant, les pratiques culturales actuelles africaines contribuent à 
appauvrir davantage les sols. Il est estimé que chaque année les sols africains sont lessivés à un rythme de $22 \mathrm{~kg} /$ ha d'azote, $2,5 \mathrm{~kg} / \mathrm{ha}$ de phosphate et $15 \mathrm{~kg} / \mathrm{ha}$ de potassium (Smaling et al., 1997).

Ainsi pour maximiser les gains de productivité de l'agriculture en Afrique, accéder aux engrais minéraux dans des conditions favorables s'avère indispensable pour atteindre le double objectif d'augmentation des rendements et de l'amélioration de la qualité de sols. La politique de promotion de l'utilisation de l'engrais minéral doit s'accompagner d'une politique de formation dans l'utilisation de la fumure organique et des pratiques de l'agriculture de conservation ainsi que de l'agroforesterie. Les voies et moyens à mettre en ouvre pour atteindre ces objectifs sont toutefois sujets de controverse. Les subventions d'intrants agricoles malgré leurs couts fiscaux élevés bénéficient à juste titre d'un regain de popularité sur le continent. L'expérience du Malawi qui augmenta substantiellement sa production alimentaire en passant d'une situation de déficit de ses besoins alimentaires de $43 \%$ en 2005 à une situation de surplus de $53 \%$ (Denning et al., 2009) principalement à la faveur des subventions ciblées sur les engrais et les semences de maïs a eu une grande force évocatrice. La généralisation dans plusieurs pays africains d'expériences de mise en œuvre de forme de subventions adaptées au marché avec des instruments tels que les bons d'achat, les aides compensatoires et les garanties partielles d'emprunt (Banque mondiale, 2007) permettrait d'améliorer la productivité de l'agriculture africaine.

\section{W Conclusion}

Définir des politiques agricoles innovantes en Afrique, c'est d'abord accepter que des ruptures soient nécessaires pour « produire plus et mieux ». En clair, une agriculture performante est nécessairement conçue dans une dynamique de co-construction, co-exécution, co-évaluation. En fait, chaque acteur des filières agricoles a une contribution à apporter. Et c'est, à n'en pas douter, l'agrégation des apports de chacun qui permet de produire des nouvelles connaissances et procédés. Il est donc important que les différents acteurs dialoguent pour mettre en place des systèmes d'innovations majeurs. L'Afrique a assez souffert des approches dirigistes, productivistes et non systémiques, du peu d'intérêt accordé à des secteurs stratégiques tels que la recherche et la vulgarisation, de la marginalisation des organisations professionnelles dans la conception et l'évaluation des politiques, d'environnement peu propice à la promotion du secteur privé.

Par conséquent, un changement de mentalité est incontournable et celui-ci doit concerner tous les acteurs publics et privés. Mais une meilleure répartition des responsabilités est nécessaire et doit être la conséquence d'un dialogue gouverné par l'excellence et impliquant tous les acteurs à tous les maillons de la chaîne de valeur. Ce continent peut et doit refuser la fatalité, car il a suffisamment d'eau, de terres, un capital humain important et beaucoup de technologies non utilisées. Il doit reconsidérer son développement rural en osant penser autrement et investir dans son futur en s'inscrivant dans une dynamique fondée sur des informations et des analyses concertées. 


\section{Références bibliographiques}

Alston J.M., Chan-Kang C., Marra M.C., Pardey P.G., Wyatt T.J., 2000. A Meta-Analysis of Rates of Return to Agricultural R\&D. ex pede Herculem? IFPRI Research Report 113. Washington, DC:IFPRI.

Anadon M., 2007. La recherche participative. Multiples regards, Presses de l'Université du Québec, $225 \mathrm{p}$.

Badiane O., 2008. Maintenir et accélérer la reprise de la croissance agricole de l'Afrique dans un contexte de fluctuation des cours mondiaux des denrées alimentaires. IFPRI. Politiques alimentaires en perspective no 9 .

Banque mondiale, 2007. Rapport sur le Développement dans le monde.

Beintema N.M., Stads G.J., 2010. Public Agricultural R\&D Investments and Capacities in Developing Countries: Recent Evidence for 2000 and beyond. Note prepared for GCARD 2010.

Blanchard M., Vall E., Chia E., 2010. Conduire une expérimentation en recherche action en partenariat co-concevoir une innovation, l'étudier, In : Innovation and Sustainable Development in Agriculture and Food (ISDA), Montpellier, France - http://hal.archives-ouvertes.fr/hal-00520255/

Chia E., 2004. Principes, méthodes de la recherche en partenariat : une proposition pour la traction animale. Revue d'élevage et de médecine vétérinaire des pays tropicaux, 57 (3-4), 233-240.

Chia E., Barlet B., Tomedi M., Pougmogne V., Mikolaseck O., 2008. Co-construction of a local fish culture system: Case study in Western Cameron, In : International Farming Systems Association (IFSA) European Symposium, Communication, Clermont-Ferrand, France 6-10 juillet 2008, 5 p.

Chia E., Deffontaines J.P., 1999. Pratiques et dispositifs de recherches face à un problème d'environnement. Nature Sciences Société, 7 (1), 31-41.

Chia E., Verspiren R., Vall E., 2009. Demande sociale, coproduction de connaissances et émancipation des acteurs. Le cas de la recherche-action-en-partenariat, In $: 2^{\mathrm{e}}$ Colloque International Francophone sur les Méthodes Qualitatives. Enjeux et stratégies, Lille, 25-26 juin 2009, 15 p.

Denning G., Kabambe P., Sanchez P., Malik A., Flor R., Harawa R., Nkhoma P., Zamba C., Banda C., Magombo C., Keating M., Wangila J., Sachs J., 2009. Input Subsidies to Improve Smallholder Maize Productivity in Malawi: Toward an African Green Revolution. PLoS Biol., 7(1), e1000023.

Douthwaite B., Keatinge J.D.H., Park J.R., 2001. Why promising technologies fail: the neglected role of user innovation during adoption. Research Policy, 30 (5), 819-836.

Ergano K., Duncan D., Adie A., Tedla A., Woldewahid G., Ayele Z., Berhanu G., Alemayehu N., 2010. Multi-stakeholder platforms strengthening selection and use of fodder options in Ethiopia: Lessons and challenges, In: Innovation and Sustainable Development in Agriculture and Food (ISDA), Montpellier, France - http://hal.archives-ouvertes.fr/hal-00522978/

Faure G., Gasselin P., Triomphe B., Temple L., Hocde H., 2010. Innover avec les acteurs du monde rural: la recherche-action en partenariat, éditions Quae - CTA, Presses agronomiques de Gembloux, $221 \mathrm{p}$.

Fisher M., Masters W.A., Sidibé M., 1998. Technical Change in Senegal's Irrigated Rice Sector: Impact Assessment under Uncertainty.WARDA Ex-Ante Impact Assessment of Rice Research Bulletin No. 98-1, Bouaké, Côte d'Ivoire, WARDA.

Hall A., 2007. Challenges to Strengthening Agricultural Innovation Systems: Where Do We Go From Here? Farmer First Revisited: 20 Years On conference at the Institute of Development Studies, University of Sussex, UK

Hawkins R., Heemsberk W., Booth R., Daane J., Maatman A., Adekunle A., 2009. Recherche agricole intégrée pour le développement. Une note conceptuelle préparée pour le FARA Challenge programme pour l'Afrique subsaharienne.

Koutou M., Vall E., 2010. Implication des acteurs locaux dans la conception d'innovations : le cas des systèmes agropastoraux du Tuy (Burkina-Faso), In: Innovation and Sustainable Development in Agriculture and Food (ISDA), Montpellier, France - http://hal.archives-ouvertes.fr/hal-00522574/

Krueger A., Schiff M., Valdes A., 1988. Agricultural incentives in developing countries: measuring the effect of sectoral and economywide policies, World Bank Economic Review, 2. 
López R., Galinato G.I., 2007. Should Governments Stop Subsidies to Private Goods? Evidence from Rural Latin America. Journal of Public Economics, 91(5-6), 1071-1094.

Masters W.A., 2005. Paying for prosperity: How and why to invest in agricultural research and development in Africa. Journal of International Affairs, 58 (2), 35-64.

Matlon P.J., Dunstan S.S., 1984. Increasing Food Production in Sub-Saharan Africa: Environmental Problems and Inadequate Technological Solutions. American Journal of Agricultural Economics, 66 (5).

Minot N., Benson T., 2009. Fertilizer subsidies in Africa: Are vouchers the answer? Issue Brief 60, International Food Policy Research Institute.

Omamo S.W., Farrington J., 2004. Policy research and African agriculture: Time for a dose of reality? ODI Natural Resource Perspectives, 90.

Scoones I., Thompson J., 2009. Farmer First Revisited: Innovation for Agricultural Research and Development, ITDG Publishing, Oxford.

Sebillote M., 2007. Quand la recherche participative interpelle le chercheur, In : La recherche participative. Multiples regards (Anadón M., ed.), PUQ, 225 p.

Smaling E.M., Nandwa S.M., Janssen B.H., 1997. Soil Fertility in Africa is at Stake, In : Replenishing Soil Fertility in Africa (Sanchez P., Buresh R., eds.), Madison, WI, Soil Science Society of America Special Publication, 51, 47-62.

United Nations, 2006. Department of Economic and Social Affairs/Population Division. World Urbanization Prospects: The 2005 Revision.

Vall E., Chia E., Andrieu N., Bayala I., 2008. Role of partnership and experimentation for the codesign of sustainable innovations: The case of the West of Burkina Faso, In : Empowerment of the rural actors. A renewal of farming systems perspectives, 8th European IFSA Symposium (Dedieu B., ed.), 6-10 July 2008, Clermont-Ferrand, France. 



\title{
Conclusion
}

\section{En route, mais laquelle ?}

\author{
Bernard Hubert, Emilie Coudel, Oliver CoOmes, \\ Christophe T. Soulard, Guy Faure, Hubert Devautour ${ }^{1}$
}

Caminante no hay camino, se hace camino al andar

Antonio Machado

\begin{abstract}
À l'instar des traditions africaines, où l'invité doit dire plusieurs fois à son hôte qu'il compte "prendre la route », avant de pouvoir s'y engager vraiment, nous sommes aujourd'hui nombreux à dire que nous souhaitons « prendre la route ». La route de l'innovation. Mais quelle innovation? Le symposium ISDA a été l'occasion de nous attarder encore un peu sur cette question, avant de nous lancer.
\end{abstract}

\section{W Revisiter les chemins habituels de l'innovation}

Si la question de l'innovation se pose aussi fortement aujourd'hui pour l'agriculture, c'est qu'il n'y a plus d'objectif simple, de chemin tout tracé. Si produire plus reste une exigence, produire mieux et autre chose - y compris des biens immatériels devient incontournable. L'agriculture doit désormais répondre à un ensemble de demandes complexes de la société : services environnementaux, insertion de populations marginalisées, différenciation des qualités, revitalisation des territoires ruraux, productions énergétiques... Autant de défis qui invitent à repenser le rôle et les fonctions mêmes du secteur agricole mais aussi la place de la recherche dans les processus d'innovation, ainsi que nous y a invité L. Busch (chap. 1).

Dans l'esprit de la modernisation des agricultures des pays industrialisés, largement diffusé dans le cadre de la révolution verte, être agriculteur consiste à produire le

1. Cette conclusion s'appuie sur les discussions qui ont eu lieu pendant le symposium ISDA, en particulier lors de la table ronde finale lorsque nous avons donné la parole à différents décideurs politiques et représentants de la société civile. Les auteurs tiennent donc à remercier l'animateur, Oliver Coomes (McGill University), et les participants de cette table ronde : Mohamed Ait-Kadi (Conseil général du développement agricole, ministère de l'Agriculture, Maroc), Pascal Bergeret (ministère de l'Alimentation, de l'Agriculture et de la Pêche, France), Dominique Chardon (agriculteur, Président de l'association Terroirs et cultures), Khalid El Harizi (FIDA - Fonds international de développement agricole), Judith Francis (CTA - Centre technique de coopération agricole et rurale), Ann Waters-Bayer (ETCProlinnova), Fabrice Dreyfus (directeur de l'IRC - Institut des régions chaudes). 
plus possible, ce qui suppose d'être innovant sur le plan technique. Mais la productivité accrue s'accompagne d'investissements conséquents, d'intégration dans des filières, réduisant le bénéfice pour l'agriculteur, et appelle souvent à plus de production, dans une fuite vers l'avant. Ce cercle vicieux de l'innovation pose question aux agriculteurs, autour du sens de leur métier. Sans compter ses effets sur l'environnement, sur le type d'alimentation, sur l'économie des territoires ruraux, qui soulèvent des contestations dans la société.

D'autres dimensions de l'innovation ont donc été intégrées, progressivement : la valorisation de la diversité biologique, la préservation de produits traditionnels, la vente directe pour renouer le lien entre agriculteurs et consommateurs, la pluriactivité qui permet de maintenir une activité agricole tout en contribuant par ailleurs à l'économie rurale. Cette expérimentation de nouveaux systèmes vient souvent des agriculteurs eux-mêmes, qui font preuve d'imagination, de créativité et d'obstination, envers et malgré les systèmes conventionnels. Mais pour que ces innovations puissent vraiment s'amplifier et s'ancrer dans la durée, pour offrir une réelle alternative, force est de constater aujourd'hui qu'elles ont besoin d'un appui de la recherche et des politiques publiques. Trop souvent, elles restent circonscrites à une poignée d'agriculteurs, elles apparaissent quelques années puis disparaissent, elles peinent à s'imposer face aux logiques dominantes encore basées sur la seule productivité du travail, de la terre ou des animaux.

Les interpellations faites à la recherche sont souvent paradoxales : soit la société s'attend à ce que la recherche lui fournisse toutes les réponses à ses problèmes ; soit la société rejette complètement la recherche, par désillusion, considérant qu'elle a «collaboré » à ce que certains considèrent comme l'impasse productiviste, ou comme étant à l'origine de nouveaux dangers pour l'environnement ou la santé, générés par les technologies issues des avancées des connaissances scientifiques. Par ailleurs, la science aborde aujourd'hui des sujets tellement complexes que les résultats peuvent paraître brouillés, ils ne s'imposent pas d'eux-mêmes à la société. Il faut alors recréer le lien subtil entre les objets que la recherche construit et la manière dont ils sont mobilisés par la société, en redéfinissant les liens science-société. Cependant, ces nouveaux objets ne sont pas seulement de plus en plus complexes, ils sont aussi parfois nouveau - pour la plupart inconnus jusqu'ici, et construits par la connaissance scientifique. N'en est-il pas ainsi du changement climatique, de l'érosion de la biodiversité, de la transgenèse, des maladies qualifiées d'émergentes, etc. ? N'est-ce pas le nouveau paradoxe d'une science en société, celui d'explorer des questions qui inquiètent ses contemporains ? Ces nouvelles questions sont souvent traitées au sein du cadre disciplinaire qui les a générées, mais ne faudrait-il pas justement les soumettre à un ensemble transdisciplinaire dépassant d'ailleurs les seuls savoirs scientifiques ? Il est important qu'un autre rôle de la recherche se définisse en lien avec la société. Une recherche dans laquelle les responsabilités sont partagées, grâce au développement de réseaux d'innovation, entre scientifiques, décideurs politiques, acteurs de la société civile. Chacun doit admettre, au-delà de ses spécialités, que les solutions n'existent pas encore, qu'il faut réussir à penser en dehors du modèle productiviste et linéaire qu'on s'était donné jusque là. Il faut définir ensemble une nouvelle vision. On ne peut pas répondre aux questions à venir à partir des recettes qui ont valu dans le cadre d'une vision remise en cause par les transformations du monde et les évolutions des modes de 
pensée sur le monde. Dans les années 1950, la vision paraissait claire, un consensus engageait toute la société vers le progrès, vers une révolution verte. L'organisation entre institutions de recherche, instituts techniques de vulgarisation, coopératives était bien réglée vers cet objectif commun. Aujourd'hui, le chemin est loin d'être aussi clair, c'est plutôt une diversité de chemins qui se dessinent. Il faut se donner les moyens de faire émerger cette diversité, consolider celle qui existe déjà au sein de différentes expérimentations, la gérer sans l'appauvrir afin d'explorer plusieurs pistes et plusieurs heuristiques porteuses de solutions futures. Cela est bien plus complexe, car il s'agit de trouver les chemins sur lesquels les différents acteurs, avec leurs différents intérêts, seront d'accord pour avancer ensemble une partie du chemin. Cela est d'autant plus difficile que celui-ci doit se construire dans un monde incertain, et qu'il est davantage attendu d'explorer et d'imaginer des pistes inédites plutôt que d'appliquer des recettes, dont on ne maîtrise pas bien ni les effets voulus ni les conséquences imprévues. C'est cela que ces dernières années nous ont appris, c'est cela également qui est débattu dans d'autres secteurs d'activité, comme ceux de l'énergie ou de l'urbanisme.

À l'issu du symposium ISDA, nous souhaitons revenir sur les interrogations soulevées dans les débats entre tous les acteurs présents, à partir des éléments de réponses qui apparaissent dans les expériences présentées :

- Comment définir une nouvelle vision ? Comment se donner de nouvelles manières de penser ? Comment déconstruire les modèles qui nous empêchent de penser différemment le développement et l'innovation ? Comment explorer de nouvelles directions?

- De quelles manières aller vers cet objectif ? Comment penser la transition vers d'autres modèles? Comment générer le changement ? Quelles connaissances construire?

- Comment s'organiser ensemble ? Comment mieux interagir pour générer des apprentissages porteurs d'innovation?

Nous présentons ici les prémisses de cette réflexion, en espérant qu'elles ouvriront sur d'autres débats, au cours d'expérimentations de terrain, au sein de programmes de recherche en partenariat, dans des forums d'échange entre acteurs.

\section{W Confronter nos visions de l'agriculture pour en explorer de nouvelles}

La remise en cause de l'hégémonie du modèle productiviste et du type d'innovation associé n'est pas une question si nouvelle. Déjà en 1993, lors du colloque international organisé par l'Inra, le Cirad et l'Orstom (devenu l'IRD) sur le thème «Innovation et sociétés : Quelles agricultures ? Quelles innovations ? », l'enjeu était de déconstruire le mythe du «transfert de technologie », critiquer les limites du topdown, où à la fois les producteurs et les consommateurs étaient trop souvent réduits à un rôle passif. Aujourd'hui, l'importance des différentes parties-prenantes est acquise, que ce soit au niveau des ONG de développement agricole passées maîtres des approches participatives, ou au niveau des grandes entreprises internationales, 
qui mettent en avant la « responsabilité sociale » et les approches multi-stakeholders qu'elles développent au sein des filières. L'enjeu n'est plus de déconstruire le modèle dominant, mais bien d'analyser comment se joue la co-existence entre différentes visions du développement. Si la plupart des opérateurs sont d'accord sur le fait qu'il faut aller vers un développement durable, les visions sous-jacentes sont contrastées, entre promesse des technologies vertes, utopie d'une décroissance écologique, ou rêve d'une société unie et solidaire.

Dans cette cohabitation entre modèles, il ne faut pas négliger l'influence des trajectoires déterminées par le passé, le phénomène de dépendance au sentier. Si le modèle productiviste reste dominant, c'est qu'il a aussi fait ses preuves, au moins dans certaines régions du monde, où l'important était d'assurer l'alimentation de populations grandissantes et la paix sociale. Un de ses avantages est de permettre une rationalisation de l'agriculture (en planifiant et mesurant), ce qui est une nécessité pour les agro-industries et une demande des investisseurs. Si ce modèle est déstabilisé aujourd'hui, c'est parce que l'organisation qu'il proposait était trop peu flexible, centré sur un objectif unique aux dépens d'autres dimensions systémiques comme l'environnement ou la santé. La complexité des défis actuels oblige à les appréhender à partir de différentes perspectives et à renouveler la manière de penser, apprenant à prendre en compte différents objectifs (sociaux, économique, environnementaux) dont la convergence ne s'impose pas. La prise en compte d'objectifs plus larges et systémiques repose sur une diversification des alliances avec les acteurs associés dans la définition du cahier des charges de la création technique et des rapports d'intéressement agencés autour d'elle. Dans cette perspective, il semble important de développer un esprit d'écologie de l'innovation, où le processus d'innovation pourrait être abordé de manière systémique, en s'intéressant aux interactions entre ses différents éléments, aux relations avec son environnement et aux transformations générées par ce complexe dynamique d'interactions et d'interdépendances.

Mais un certain nombre de questions subsistent. Peut-on combiner différents modèles d'innovation pour penser la diversité, ou certains modèles restent-ils incompatibles ? Peut-on construire à partir de ce qui a déjà été développé, ne pas perdre complètement tous les repères qui existent déjà? Doit-on s'orienter vers des nouveautés incrémentales (petit à petit), ou des nouveautés radicales (tout changer d'un coup) ? Il est clair que les acteurs de chaque domaine devront trouver leurs propres réponses, mais il est important d'avoir conscience que c'est une question d'équilibre entre une nouveauté créatrice et une nouveauté déstabilisatrice.

Car l'innovation est aussi une forme de remise en cause de ce qui existe déjà, comme le montre J. Santilli (chap. 8), et qui dit remise en cause dit résistance. Toute innovation fera apparaître des résistances qui, sans elle, seraient restées cachées... Peut-on mieux anticiper, éviter les risques, les désordres et les dégâts générés par de fausses pistes et choisir, à temps, les voies d'innovation qui fédéreront, qui mobiliseront, au-delà des différences? L'innovation peut être pensée comme un rapport de force entre différentes visions, et suppose de s'interroger sur ces visions, voire même de chercher à en susciter de nouvelles, qui faciliteront la recherche d'objectifs communs. Débattre de ces visions est le premier pas vers l'identification des critères de choix du processus de changement à engager. 
Si le bilan du modèle passé commence à être relativement bien dressé, selon les secteurs, les régions, pointant l'exclusion générée, les problèmes environnementaux, ou les impasses économiques, les analyses des nouvelles propositions restent encore à renforcer, pour appuyer les choix de la société. Il faut se donner les moyens d'aller à l'encontre des idées reçues, afin de concevoir des alternatives réellement argumentées et dont on connaît mieux le potentiel et les limites, et pas seulement des propositions politiquement correctes et bien pensantes : les coopératives apportent-elles réellement plus de sécurité aux petits producteurs ? Les biotechnologies entraînentelles nécessairement des phénomènes de concentration des richesses et d'inégalités? De nombreuses expériences locales (mais parfois aussi régionales, voire nationales) apportent de nouvelles propositions et méritent d'être analysées de manière plus approfondies, comparées, pour en tirer des pistes génériques.

Mais il s'agit également de s'engager dans des démarches d'exploration et pas seulement dans l'exploitation de ce qu'on sait déjà en l'aménageant à la couleur du temps. Il faut apprendre à explorer ce qu'on ne sait pas, à imaginer, à créer ! La prospective, par exemple, peut constituer un outil d'exploration des changements possibles : elle vise en effet bien plus - à travers la caractérisation d'états futurs, craints ou souhaités - à aider à l'identification des variables qui conditionnent le choix de tel ou tel chemin à prendre dès maintenant si on veut aller plutôt vers tel état futur que tel autre.

\section{W Engager les transitions pour générer le changement}

L'avènement du participatif a provoqué une diversification méthodologique ces 15 dernières années, laissant sur le bord du chemin les recettes toutes faites pour s'orienter vers des méthodes permettant d'accompagner la prise de décision collective. S'il existe aujourd'hui de nombreux outils et méthodes pour aider à construire des diagnostics partagés d'une situation (en quoi est-elle jugée insatisfaisante ? critique ? en ne se contentant pas d'analyses simplistes en termes de relations de causes à effets), et explorer les futurs possibles et l'état désiré par l'ensemble des acteurs (même si le résultat de ces processus de concertation est loin d'être garanti, car il dépend des intérêts, parfois forts divergents, des participants !), le principal défi actuel est d'aider à définir comment passer de la situation actuelle à la situation imaginée.

Les processus de transition ont depuis longtemps été étudiés, décortiqués, par les historiens, les agronomes, les sociologues, mais leurs ressorts profonds restent souvent mystérieux, entre déterminismes contextuels et leaders déterminants. Dans les faits, on a différents états de départ, on souhaite aller vers des états nouveaux, différentes trajectoires sont possibles, et elles-mêmes sont déterminées par les transitions qui sont en cours. Comment dès lors accompagner les transitions ? Comment générer les dynamiques souhaitables, quels changements inciter, comment s'adapter en fonction des incertitudes qui se dévoilent ? Il s'agit de se lancer dans un réel processus de «conception innovante » et pas seulement de formes de « conception réglée » (Le Masson et al., 2008), familière de vieilles recettes qu'il suffirait de renouveler. 
Les entrepreneurs innovants ont leur importance, encore faut-il les encourager, leur permettre de développer leurs bonnes idées, leur créativité, comme le pointent justement A. Hall et K. Dorai (chap. 3). Les niches d'innovation, endroits où innover en toute sécurité, en dehors des contraintes du modèle dominant, peuvent permettre l'émergence de cette créativité. Mais de là à ce que ces innovations soient capables de générer une transition vers un autre régime (Kemp et al., 2007), s'élève tout un ensemble de questions d'échelles, d'institutions, de capacités individuelles et collectives, de barrières physiques, culturelles.

Ainsi, dans le domaine agricole, se pose la question des réelles capacités d'émergence de nouveaux choix technologiques (avec tout ce qu'ils supposent comme changements sociaux, économiques, d'aménagement de l'espace, etc.), ainsi que le soulève J.-M. Meynard (chap. 4). Il pourra s'avérer difficile de sortir des choix passés tant ils sont intégrés, non seulement dans les solutions techniques actuelles (mécanisation, engrais, pesticides, génétique, etc.) mais aussi dans les systèmes cognitifs (savoirs et savoir-faire, représentations de la nature, des nuisances, des paysages, etc.) et de valeurs des principaux acteurs impliqués. Ne risquons-nous pas d'être pris au piège de la rationalisation technique, sorte de lock in, ainsi que l'ont connu d'autres secteurs d'activité ? Comment mieux analyser ces situations de blocage (dead-lock) et réussir à les dépasser ?

Le vrai travail méthodologique est bien là, il est temps de s'en emparer : quels sont les concepts, les connaissances et les compétences qui pourront nous permettre d'envisager la transition? Non pas de la déterminer à l'avance, mais d'être capable de la choisir progressivement, chemin faisant, de s'adapter pour arriver éventuellement à l'horizon envisagé, ou, plus probablement, définir sans cesse de nouveaux horizons à atteindre, en les envisageant divers. Il s'agit d'aller vers autre chose que des objectifs sûrs, qui n'amènent pas de grande reconsidération des pratiques et valeurs actuelles. Il faut prendre conscience qu'il n'y a pas de solution optimale, mais se donner les moyens d'évaluer les différentes options au fur et à mesure qu'elles apparaissent, décider à partir des trade-offs en soumettant les choix aux différentes parties prenantes. C'est cette connaissance critique, de critères, de seuils, cette compétence d'évaluation, de remise en cause, qu'il faut faire émerger. Il s'agit d'un apprentissage que toute société doit opérer, car les choix devront être pris par les décideurs politiques, guidés par la recherche, en fonction des attentes exprimées par la société. C'est en créant les conditions de cet apprentissage social (Röling, Wagemakers, 1998), de connaissances et de valeurs construites et choisies consciemment par la société, qu'on se donnera les conditions des transitions vers d'autres modèles de développement.

L'apprentissage social n'est pas quelque chose de lisse, il ne surgit pas du consensus. Il implique un débat sociétal, une confrontation d'opinions et de visions. Comment les concepts et les connaissances orientant la transition seront-elles produites, légitimées? Au sein de quels réseaux, de quelles institutions dominantes ou marginalisées, grâce à quel processus ? Que ce soit les concepts qui orientent la pensée, les objets qui focalisent l'attention, les indicateurs et critères d'évaluation, les seuils choisis, les étapes dessinées, toute cette production cognitive nous invite à une réflexion critique et récursive, qui alimentera progressivement l'apprentissage social. 


\section{" Innover, c'est d'abord interagir pour apprendre}

Comment rendre cet apprentissage social concret ? Quels types d'interactions et d'organisation généreront l'innovation ? Travailler ensemble, au-delà de nos différences, représente toujours l'un des principaux défis. L'innovation, on l'a assez dit, ne concerne pas que les chercheurs. Il s'agit donc d'échanger entre agriculteurs, acteurs ruraux, décideurs politiques, scientifiques, et avec toute la diversité qu'il y a derrière chaque «catégorie ». Heureusement, des institutions existent pour faciliter et formaliser ces échanges, mais on s'empêtre parfois dans les routines, oubliant l'objectif principal. Il faut se donner les moyens de récréer du dialogue, de la souplesse, pour permettre à la créativité collective de s'exprimer. Être capable de créer ensemble, de proposer de nouvelles solutions. Pour cela, nous avons besoin de concepts intégrateurs, qui amènent les gens à discuter ensemble au sein de véritables systèmes d'innovation, tels que nous les présentent B. Triomphe et R. Rajalahti (chap. 2). Ceux-ci se développent en général au sein de filières, de territoires, qui présentent l'avantage de problématiser, de focaliser les discussions sur un « problème » précis. Mais on a sans doute aussi beaucoup à gagner de discussions entre différents types de filières, entre différentes échelles.

Ces discussions, on le remarque, se font souvent en dehors des institutions, aux marges des systèmes formalisés, là où il y a de la place pour la créativité, de la spontanéité. La surprise doit pouvoir émerger, la prise de risque doit être possible. C'est pourquoi les réseaux, informels ou formels, les espaces d'échange, ont toute leur importance. Les intermédiaires, les médiateurs, les passeurs, qui font le pont entre institutions, entre groupes sociaux, ont un rôle fondamental, si justement ils n'alourdissent pas en sclérifiant les processus qu'ils sont sensés faciliter. Ils traduisent les idées d'un groupe à l'autre, trouvent les intérêts communs, « hybrident » les savoirs, enrôlent les personnes de groupes différents. Ils créent du lien entre différents mondes, ouvrent de nouveaux espaces d'échange, là où les nouvelles idées, potentielles innovations, peuvent surgir.

Cela ne veut pas dire que les institutions plus formelles n'ont pas leur importance. L'État, les entreprises privées, les associations, etc., peuvent créer des conditions privilégiées pour faire émerger des innovations, peuvent ouvrir des espaces nouveaux, comme le montrent K. Hussein et K. El-Harizi (chap. 9). Surtout, une fois que les innovations ont surgi, ces institutions formalisent les liens entre acteurs par des contrats, des statuts, des accords, des ressources, pour permettre à cette innovation de se répandre plus largement. Les filières et les opérateurs privés ont un rôle particulièrement important dans ce type d'up-scaling, offrant une certaine sécurité dans la prise de risque que peut représenter une innovation. Mais il est important qu'elles soient construites collectivement, pour éviter des effets d'exclusion, comme le souligne E. Biénabe, C. Bramley et J. Kirsten (chap. 6).

On le voit bien, des rôles différents s'affirment, entre les créateurs et les formalisateurs (Rogers, 1962), renvoyant aux capacités des uns et des autres à remplir leur rôle. Comment encourager les « avantages comparatifs » des différents acteurs, les aider à renforcer leurs compétences dans un sens ou dans l'autre ? Comment permettre un alignement entre les ressources (financières, sociales, institutionnelles) vers les différents types d'acteurs? 
Si ces questions se posent, c'est que l'innovation est souvent à l'origine de conflits de répartition, de phénomènes d'exclusion. Il existera toujours des acteurs en marge des processus d'innovation, là n'est pas le problème. Mais la question est celle du choix qu'ont les acteurs à participer ou non à ce processus. Comment réduire les asymétries pour faciliter des échanges plus égaux ? Femmes, jeunes, immigrés, comment créer les conditions de leur participation dans ces processus d'innovation ? Comme le montre bien D. Requier-Desjardins (chap. 5), on ne peut déconnecter la réflexion sur les politiques pour l'innovation des autres politiques de garantie des droits fondamentaux, d'éducation, de conditions de santé, d'accès aux infrastructures. Les « enrôleurs » du processus d'innovation peuvent également être déterminants, qu'ils soient animateurs d'une association, d'un territoire, entrepreneurs, chercheurs, car ce sont eux qui peuvent inviter les acteurs généralement oubliés, les convaincre de leur importance au sein du groupe, renforçant leur capacités de proposition et d'adaptation, et finalement de « co-gouvernance » du processus d'innovation, vers une « démocratisation » de ces processus. Les jeux de pouvoir ne disparaissent pas pour autant, mais ils peuvent être en partie rééquilibrés ou en tout cas rendus explicites. A. Torre et F. Wallet (chap. 7) soulignent ainsi l'importance des conflits au cours du processus d'innovation.

\section{"Le chercheur, acteur du changement?}

Au terme de ce questionnement, sur les visions, les transitions à engager et les interactions pour y arriver, il importe de se poser la question : Comment les chercheurs peuvent-ils se situer dans ces collectifs et démarches d'innovation ? La créativité n'est pas toujours le propre de l'activité de recherche et c'est bien dommage. Néanmoins la recherche peut avoir un rôle significatif, si elle est capable, un tant soit peu, de se remettre en question. Face aux interrogations, son rôle n'est pas tant d'apporter des solutions toutes faites, mais de problématiser, éclairer les décisions et appuyer la co-construction de nouveaux savoirs.

La problématisation peut s'engager au travers d'un travail de dénaturalisation/régénération, par la déconstruction des évidences, par la révélation de faits connus et de faits non perçus, et par la création de connaissances et de relations nouvelles, de façon à s'interroger sur les normes, les concepts, les critères d'efficacité des actions engagées pour les remettre en cause et les transformer en fonction des changements en cours.

Le processus de recherche n'est pas dissociable du processus de décision : l'investigation est constituante de la conception de solutions, conduisant à des choix explicites. C'est en assumant notre rôle d'éclairer les enjeux de différents choix qu'on peut redonner son rôle au politique, dans son sens large. Redonner le pouvoir de décision aux différents acteurs, au sein d'arènes ouvertes. Car face aux modèles de développement dominants, autoproclamés et justifiés comme des évidences, il est bien difficile pour les citoyens de réagir et de concevoir des alternatives qui ne se fondent pas quasi-exclusivement sur des positions idéologiques. Il peut être attendu de la science qu'elle contribue à fournir une variété de modèles entre lesquels un processus démocratique peut déboucher sur des choix, tout en rappelant que les 
réponses apportées par la science sont façonnées par des cadres théoriques construits (les paradigmes) et qu'elles ne sont pas toujours définitives.

La recherche peut également aider à pondérer les relations entre formes de savoirs, en les situant dans leur contexte, dans leur domaine d'application et dans leur légitimité. Ce travail est indispensable à la discussion entre points de vue, pour éviter de mettre en exergue un seul côté. Cela peut permettre aux gens de comprendre leurs propres représentations et les représentations des autres, pour les dépasser et être capable de construire ensemble de nouveaux concepts et savoirs pour faire face à leurs problèmes. En s'engageant, avec les autres, dans l'action collective, la recherche s'implique dans un espace de reconstruction simultanée des savoirs des acteurs et des savoirs des chercheurs, soit en révélant des savoirs qui existent déjà, soit en produisant de nouveaux savoirs. Cette reconstruction concerne d'une part les concepts qui permettent de penser ensemble les objectifs, les produits, les tâches à mener; et de l'autre, les connaissances qui permettent de mettre en œuvre concrètement ces objectifs.

Par ce tryptique problématiser-éclairer-interagir, les chercheurs peuvent appuyer la réflexivité au cours du processus, de façon à permettre une réflexion critique sur les objectifs et les moyens mis en œuvre, une évaluation continue, qui est la condition d'un apprentissage social. Pour cela, il faut construire de nouveaux critères de performances susceptibles d'évaluer ces nouveaux modèles en regard des multiples paramètres à prendre en compte (productivité, emploi, services écosystémiques, changement climatique, développement des espaces ruraux, etc.). De tels critères seront indispensables si on souhaite mettre de nouveaux modèles de production agricole en politique et les administrer, et faire en sorte que différents types d'agriculture se complètent plutôt que d'attendre de les aligner sur un modèle unique...

Le changement relève d'une dynamique sociale dans laquelle se transforment non seulement les perspectives et les objets d'intérêt, mais également chacun des acteurs impliqués dans cette dynamique collective. Les processus d'apprentissages croisés qui s'y déroulent sont le fait des changements dans ce système d'interaction entre porteurs d'enjeux, aussi bien qu'entre eux-mêmes et les objets qui les concernent. Comme le montrent P. Seck, A. Diagne et I. Bamba (chap. 10), c'est sur la capacité des chercheurs à s'engager dans un tel processus d'apprentissage que reposera la réussite de leur implication, et donc, tout simplement, l'intérêt de cette implication. Cette capacité n'est pas donnée à tous selon leur culture, leur position dans les groupes constitués, leur aptitude à s'engager dans un processus dont le produit final n'est pas connu d'entrée de jeu. Dans une démarche exploratoire, les éléments pris en compte dans la décision sont le résultat du processus et non son point de départ ; elle repose sur l'exploration des mondes possibles par la discussion conjointe des faits - pris comme des construits - et des valeurs. C'est dans de tels dispositifs mêlant des rationalités, des connaissances, des faits, des codes, des normes, des valeurs et des acteurs divers au sein de réseaux hybrides, que s'élabore l'innovation. Ce n'est pas ainsi que travaillent habituellement les chercheurs sur la base d'un paradigme distinguant faits et valeurs. Combien d'entre nous sommes effectivement prêts à nous engager dans des dispositifs d'apprentissage, dont nous sortirons forcément transformés, du fait de l'inséparabilité connaissances-relations ? 
Et comment alors être aidé dans ce cheminement risqué, comment identifier des procédures qui garantissent la rigueur de nos apports et la légitimité de nos alliances ? Probablement, ainsi que cela a pu se faire à l'occasion de ce symposium, en s'inspirant d'une diversité d'expériences, en apprenant de nos erreurs, en ne nous contentant pas des success stories, mais en analysant avec encore plus d'attention les failures. Et bien sûr, il faut poursuivre le travail sur la construction des cadres conceptuels, des démarches et des méthodes qui garantissent le bon déroulement de ce type d'implication dans les dynamiques de changement.

Ce devrait être là des domaines de recherche à développer sérieusement au sein des établissements engagés dans des recherches pour l'innovation et le développement, afin qu'elles ne soient pas laissées aux seules initiatives des chercheurs qui en acceptent les règles si difficiles à définir. Et, ici également, les voies de la réussite sont multiples et doivent être adaptées à une diversité de situations, de problèmes, mais aussi de cultures scientifiques... Gageons que des symposium comme ISDA 2010 soulignent l'intérêt de renforcer les recherches sur la conception des systèmes d'innovation et sur les formes et les modalités d'implication des chercheurs dans les dispositifs d'action collective qui génèrent le changement ! Alors en route, mais donnons-nous rendez-vous pour faire le point d'ici quelques années !

\section{" Références bibliographiques}

Kemp R., Loorbach D., Rotmans J., 2007. Transition management as a model for managing processes of co-evolution towards sustainable development. The International Journal of Sustainable Development and World Ecology, 14 (1), 78-91.

Le Masson P., Weil B., Hatchuel A., 2008. Les processus d'innovation, conception innovante et croissance des entreprises, Hermès Lavoisier, Paris, 471 p.

Rogers E.M., 1962. Diffusion of innovations, Free Press, New York.

Röling N., Wagemakers M., 1998. Facilitating Sustainable Agriculture: Participatory Learning and adaptive management in times of environmental uncertainty, Cambridge University Press, Cambridge, New York, Melbourne. 


\section{Sigles}

AARINENA Association of Agricultural Research Institutions in the Near East and North Africa

AFD Agence française de développement

AIR Agro-industries rurales

ANT Actor-Network Theory

ARIMNet Agricultural Research In the Mediterranean Network

ASARECA Association for Strengthening Agricultural Research in Eastern and Central Africa

BERD Banque européenne pour la reconstruction et le développement

BRAC Bangladesh Rural Advancement Committee (Comité du Bangladesh pour le progrès rural)

$\mathrm{CDB} \quad$ Convention sur la diversité biologique

CGDA Conseil général du développement agricole (Maroc)

CIAL Comité de recherche agricole local (Honduras)

CIAT Centre international d'agriculture tropicale

Cirad Centre de coopération internationale en recherche agronomique pour le développement

Cirdes Centre international de recherche-développement sur l'élevage en zone Subhumide

CNUCED Conférence des Nations unies sur le commerce et le développement

CONDESAN Consortium for the Development of the Andes Ecoregion

Coraf Conseil Ouest et Centre africain pour la recherche et le développement agricoles

CRDI Centre de recherches pour le développement international

CTA Centre technique de coopération agricole et rural

DUI Doing, Using, Interacting

ENA École nationale d'administration de Rabat

ENFI École nationale forestière d'ingénieurs

ETC Educational Training Consultancy

FAO Organisation des Nations unies pour l'alimentation et l'agriculture

FARA Forum for Agricultural Research in Africa

FEM Fonds pour l'environnement mondial

FIDA Fonds international de développement agricole

FSR Farming System Research 


\begin{tabular}{|c|c|}
\hline GAPs & Good Agricultural Practices \\
\hline GCARD & Conférence mondiale sur la recherche agronomique pour le développement \\
\hline GCRAI & Groupe consultatif pour la recherche agricole internationale \\
\hline GCRN & Gestion concertée des ressources naturelles \\
\hline IAV & Institut agronomique et vétérinaire Hassan II \\
\hline ICAR & Indian Council for Agricultural Research \\
\hline ICCROM & $\begin{array}{l}\text { Centre international d'études pour la conservation et la restauration } \\
\text { des biens culturels }\end{array}$ \\
\hline ICRISAT & $\begin{array}{l}\text { Institut international de recherche sur les cultures des zones tropicales } \\
\text { semi-arides }\end{array}$ \\
\hline IED-Afrique & Innovation environnement développement \\
\hline IFOAM & $\begin{array}{l}\text { International Federation of Organic Agriculture Movements (Fédération } \\
\text { internationale des mouvements de la bio) }\end{array}$ \\
\hline IIRR & Institut international de reconstruction rurale \\
\hline ILRI & International Livestock Research Institute \\
\hline INGC & Institut national des grandes cultures (Tunisie) \\
\hline Inra & Institut national de la recherche agronomique \\
\hline IRD & Institut de recherche pour le développement \\
\hline IRESA & Institution de la recherche et de l'enseignement supérieur agricole (Tunisie) \\
\hline ITPGRFA & International Treaty on Plant Genetic Resources for Food and Agriculture \\
\hline NAIP & National Agricultural Innovation Project \\
\hline OCDE & Organisation de coopération et de développement économiques \\
\hline ONG & Organisations non gouvernementales \\
\hline PMCA & Participatory Market Chain Approach \\
\hline PNUD & Programme des Nations unies pour le développement \\
\hline PPP & Partenariats public-privé \\
\hline PRF & Projets de recherche fédérés \\
\hline PSDR & Pour et sur le développement régional (Languedoc-Roussillon) \\
\hline RAP & Recherche-action-en-partenariat \\
\hline ROPPA & $\begin{array}{l}\text { Réseau des organisations paysannes et des producteurs agricoles de l'Afrique } \\
\text { de l'Ouest }\end{array}$ \\
\hline SACCAR & $\begin{array}{l}\text { Southern African Centre for Cooperation in Agricultural Research } \\
\text { and Training }\end{array}$ \\
\hline SIA & Systèmes d'innovation agricoles \\
\hline Sipam & Systèmes ingénieux du patrimoine agricole mondial \\
\hline SNRA & Systèmes nationaux de recherche agricole \\
\hline SPL & Systèmes locaux de production \\
\hline SRI & Système de riziculture intensive \\
\hline Syal & Système agroalimentaire localisé \\
\hline TTO & Technology Transfer Offices \\
\hline
\end{tabular}


UICN Union internationale pour la conservation de la nature

Unesco Organisation des Nations unies pour l'éducation, la science et la culture

UNU Université des Nations unies

UPAC Unité de recherche et d'apprentissage collectifs

UTAP Union tunisienne de l'agriculture et de la pêche 



\section{Liste des auteurs}

Ibrahima Bamba a obtenu un doctorat en économie agricole à l'Université du Kentucky en 2004 ainsi qu'une maîtrise d'économie à l'Université d'Aix-Marseille. Il travaille actuellement pour le FIDA, après une période auprès de AfricaRice de 2007 à 2011.

FIDA, Rome, Italie

i.bamba@ifad.org

Estelle BiEnabe est économiste agricole au Cirad, spécialisée dans l'accès aux marchés, la dynamique de qualité et l'analyse des politiques environnementales.

Cirad, Montpellier, France

estelle.bienabe@cirad.fr

Cerkia Bramley est chercheur à l'unité de Droit du développement international de l'Université de Pretoria. Avocate expérimentée en économie agricole, elle s'intéresse notamment à des sujets en rapport avec le droit de la propriété intellectuelle et de l'alimentation.

Department Agricultural Economics and Rural Development, University of Pretoria, South Africa

cerkia.bramley@up.ac.za

Lawrence Busch est professeur émérite de sociologie à l'Université de l'État du Michigan. Il a plus de 150 publications à son actif, dont Standards: Recipes for Reality (MIT Press, 2011). Il est par ailleurs membre de l'Académie d'agriculture de France.

Department of Sociology, Michigan State University, USA

Lbusch@msu.edu

Oliver T. Coomes est professeur de géographie à l'Université McGill. Ses recherches portent sur les moyens de subsistance des paysans, la pauvreté et les changements environnementaux chez les peuples traditionnels de l'Amazonie et d'autres régions de l'Amérique tropicale.

Department of Geography, McGill University, Montreal, Canada oliver.coomes@mcgill.ca

Emilie Couber est agro-économiste au Cirad. Elle s'intéresse aux capacités des agriculteurs à participer aux processus de gouvernance territoriaux, particulièrement ceux liés à une transition environnementale au Brésil.

UR Green, Cirad, Belem, Brésil

emilie.coudel@cirad.fr

Hubert Devautour, directeur-adjoint du département scientifique Environnement et sociétés du Cirad, est économiste agro-alimentaire. Il a travaillé sur la 
valorisation des productions de l'agriculture familiale et les processus d'innovation dans l'artisanat alimentaire en Afrique.

Département ES, Cirad, Montpellier, France

hubert.devautour@cirad.fr

Aliou Diagne est spécialisé en économie agricole et en économie. Il est membre du comité de rédaction de l'African Journal of Agricultural and Resource Economics (depuis 2005) et de Food Security (depuis 2009). Il est actuellement à la tête du programme « Policy, Impact Assessment and Innovations Systems » de AfricaRice (Centre du Riz pour l'Afrique).

AfricaRice, Cotonou, Bénin

a.diagne@cgiar.org

Kumuda DoraI est chargée de programme à LINK Ltd, une société proposant des services de recherche et de conseil en innovation pour le développement et qui a débuté sous la forme d'un projet conjoint de l'UNU-MERIT et de la FAO. Elle s'intéresse notamment aux moyens d'aller au-delà des idées conventionnelles en matière d'assistance au développement. Son travail à LINK l'amène également à étudier de nouvelles sources d'innovation pertinentes sur le plan social, y compris les tendances émergentes en matière d'entrepreneuriat hybride.

LINK Ltd., Brighton, UK

kumuda.dorai@innovationstudies.org

Khalid El Harizi, spécialiste en économie agricole, a rejoint le FIDA en 1991. Actuellement chargé du programme Cambodge, il s'intéresse de près au rôle potentiel de l'innovation dans l'autonomisation des populations rurales pauvres, domaine dans lequel il a acquis une solide expérience.

FIDA, Rome, Italy

k.elharizi@ifad.org

Guy Faure, directeur adjoint de l'UMR Innovation, est économiste au Cirad. Ses recherches portent sur les systèmes agricoles, sur les services de conseils et les organisations de producteurs, par la mobilisation d'approches de recherche-action en Afrique et Amérique latine.

Cirad, UMR Innovation, Montpellier, France

guy.faure@cirad.fr

Andy Hall est directeur de LINK Ltd, une société qui propose des services de recherche et de conseil en matière d'innovation pour le développement. Auparavant directeur de recherche à l'UNU-MERIT, il est actuellement professeur invité à l'Open University au Royaume-Uni. Il s'intéresse notamment à l'application des idées systémiques au développement agricole et au renforcement des capacités. Ses travaux en cours explorent de nouvelles sources d'innovation pertinentes sur le plan social au sein des économies émergentes.

LINK Ltd., Brighton, UK

andy.hall@innovationstudies.org

Bernard Hubert, directeur de recherche à l'Inra et directeur d'études à l'EHESS, s'intéresse aux sciences sociales et à leur apport épistémologique en regard des sciences du vivant. Il préside Agropolis International à Montpellier depuis avril 2009. Agropolis International, Montpellier, France

bernard.hubert@agropolis.fr 
Karim Husseın a travaillé plus de 20 ans sur les politiques de développement et leur application pratique, et plus particulièrement sur les moyens de subsistance, la pauvreté, l'accès à l'innovation, les services agricoles suscités par la demande, la sécurité alimentaire, la participation, les organisations de producteurs et les situations de conflit. Il a coordonné des programmes de l'OCDE et du FIDA pour la promotion de partenariats pour l'innovation agricole en Afrique.

OCDE, Paris, France

karim.hussein65@yahoo.co.uk

Jean-Marc Meynard est directeur de recherche à l'Inra. Responsable du département de recherche Sciences pour l'action et le développement de 2003 à 2012, ses recherches portent sur la conception de systèmes de culture innovants, dans l'objectif de réduire les intrants et de concilier performances productives et environnementales. Il est depuis 2008 président du Conseil scientifique français pour l'agriculture biologique.

Inra, Département SAD, Versailles, France

meynard@grignon.inra.fr

Johann KIRSTEN est professeur et directeur du département d'Économie agricole, de vulgarisation et de développement rural à l'Université de Pretoria. Il a été viceprésident de l'Association internationale des économistes agricoles de 2006 à 2009. Department of Agricultural Economics, Extension and Rural Development,

University of Pretoria, South Africa

johann.kirsten@up.ac.za

Riikka RaJalahtı est expert en agriculture, spécialisée dans les systèmes d'innovation agricoles, au département de l'Agriculture et du développement rural de la Banque mondiale. Elle a pour tâche principale de promouvoir l'opérationnalisation de l'approche par les systèmes d'innovation agricoles dans le cadre des opérations de prêt de la Banque en mettant à profit les bonnes pratiques, le transfert de connaissances et le cross-support direct avec les régions, à l'échelle mondiale. Elle a récemment été chargée de diriger l'élaboration d'un compendium sur l'investissement dans les systèmes d'innovation agricoles (Agricultural Innovation Systems. An Investment Sourcebook).

The World Bank, Department of Agriculture and Rural Development,

Washington DC, USA

rrajalahti@worldbank.org

Denis RequiER-DesJaRdins est attaché à l'IEP de Toulouse, après avoir travaillé aux universités de Constantine (Algérie), Abidjan (Côte d'Ivoire), Lyon, Rennes et Versailles. Ses recherches portent sur l'économie du développement, le développement rural (en particulier en Amérique latine), les systèmes agroalimentaires localisés et les migrations, les transferts et le développement.

Institut d'études politiques de Toulouse, France

denis.requier-desjardins@univ-tlse1.fr

Juliana SANTILLI est avocate, procureur et chercheur, spécialisée dans le droit du patrimoine environnemental et culturel et dans les politiques publiques. Elle est l'auteur de l'ouvrage Agrobiodiversity and the Law: regulating genetic resources, food security and cultural diversity (Earthscan, 2012). 
Ministério Público da União, Brasilia, Brasil juliana.santilli@superig.com.br

Papa Abdoulaye SEck est directeur de recherche, spécialisé dans l'analyse et la stratégie des politiques agricoles. Il est membre de l'Académie des sciences du Sénégal. Depuis 2006, il est le directeur général d'AfricaRice, un centre de recherche du consortium du CGIAR.

Africa Rice Center, Cotonou, Bénin

p.seck@cgiar.org

André Torre est directeur de recherche à l'Inra et à AgroParisTech. Il enseigne dans plusieurs universités françaises. Rédacteur en chef de la Revue d'Économie Régionale et Urbaine, il a été président de la section francophone de l'European Regional Science Association. Il a publié de nombreux ouvrages et articles sur des sujets en lien avec la proximité, l'innovation et le développement régional.

UMR SAD-APT, Agro Paristech, Paris, France torre@agroparistech.fr

Christophe-Toussaint Soulard, directeur de l'UMR Innovation à Montpellier, est géographe, ingénieur de recherche à l'Inra. Il étudie les formes innovantes d'agriculture dans les territoires ruraux et périurbains en Méditerranée.

Inra, UMR Innovation, Montpellier, France

Soulard@supagro.inra.fr

Bernard Triomphe est agronome des systèmes avec 25 ans d'expérience dans les pays en voie de développement. Il s'intéresse notamment à l'évaluation des systèmes agraires et des processus d'innovation, et à la conception d'innovations dans le cadre de partenariats multi-acteurs.

Cirad, UMR Innovation, Montpellier, France

bernard.triomphe@cirad.fr

Frederic WaLlet est économiste, il travaille principalement sur l'analyse de l'innovation institutionnelle dans les politiques de développement rural et dans la gouvernance territoriale. Son second thème de recherche concerne l'évolution des mécanismes de régulation des produits comportant une indication géographique et leurs effets sur les territoires.

Inra, UMR SADAPT, Paris, France

wallet@agroparistech.fr

\section{" Auteurs des encadrés}

Araya Rodolfo, Universidad Costa Rica, San José, Costa Rica

Bazile Didier, Cirad, UPR 47/Green, Instituto de Geografia, PUC Valparaíso, Chile

Blanchard Mélanie, Cirdes, 01 Bobo-Dioulasso, Burkina Faso

Bolo Maurice Ochieng, The Open University, Walton Hall, Milton Keynes, MK7 6AA, United Kingdom

Bonnal Philippe, Cirad, UMR Politiques et Marchés, Montpellier, France 
Boucher François, Cirad, UMR Innovation, IICA-México, Mexique

Brun Virginie, IICA-México, Mexique

Chebrolu Shambu Prasad, Xavier Institute of Management, Bhubaneswar, Orissa, India

Chia Eduardo, Inra Supagro Cirad, UMR Innovation, Montpellier, France

De Bon Hubert, Cirad, UPR Hortsys, Montpellier, France

de Carvalho Noya Eliane, Instituto Agronomico de Pernambuco IPA, Brésil

Eloy Ludivine, UMR Art-Dev 5281, Montpellier, France

Emperaire Laure, UMR 208 Patrimoines locaux

Ergano Kebebe, International Livestock Research Institute, Addis Ababa, Ethiopie

Errahj Mostafa, École nationale d'agriculture de Meknès (ENA), département Ingénierie du développement, Meknès, Maroc

Fernandez-Baca Edith, The Consortium for the Sustainable Development of the Andes Eco-Region, Lima, Pérou

Ghezán Graciela, EEA INTA, Balcarce, Argentine

Hocdé Henri, Cirad, Avenue Agropolis, Montpellier, France

Jongerden Joost, Department of social sciences, Critical technology Construction, Wageningen, The Netherlands

Kadiri Zakaria, Laboratoire Méditerranéen de Sociologie (Lames), UMR G-eau, Aix en Provence, France

Kahane Rémi, GlobalHort, Arusha, Tanzania

Khaldi Raoudha, Institut national de la recherche agronomique de Tunisie, Ariana, Tunisie

Koutou Mahamoudou, Cirdes, Bobo-Dioulasso, Burkina Faso

Kupper Marcel, Cirad, UMR G-Eau, Avenue Agropolis, Montpellier, France

Lemmens Pieter, Centre for Methodical Ethics and Technology Assessment (META), Wageningen University, The Netherlands

Loconto Allison, Department of Sociology, Michigan State University, USA

Majella Bezerra Lopes Geraldo, Instituto Agronomico de Pernambuco IPA, Brésil

Mateos Mónica, Fac. Ciencias Agrarias, Balcarce, Argentine

Parrot Laurent, Cirad, UPR Hortsys, Montpellier, France

Paz-Montoya María, The Consortium for the Sustainable Development of the Andes Eco-Region, Lima, Pérou

Pédelahore Philippe, Cirad, UMR Innovation, Yaoundé, Cameroon

Piraux Marc, Cirad, UMR Tetis, Université fédérale de Campina Grande, Brésil

Rey-Valette Hélène, Université Montpellier 1, UMR Lameta, Montpellier, France

Rosas Juan Carlos, Escuela Agrícola Panamericana, Honduras 
Roux Bernard, CESAER/Agrosup Dijon, France

Ruivenkamp Guido, Wageningen University, Department of social sciences, Critical technology Construction, Wageningen, The Netherlands

Simbua Emmanuel, Tea Research Institute of Tanzania, Dar es Salaam, Tanzania

Thomet Max, CET-Sur, Temuco, Chili

Vall Eric, Cirad, Avenue Agropolis, Montpellier, France

Yañez Natalia, The Consortium for the Sustainable Development of the Andes Eco-Region, Lima, Pérou 